\title{
Corner effects for oblique shock wave/turbulent boundary layer interactions in rectangular channels
}

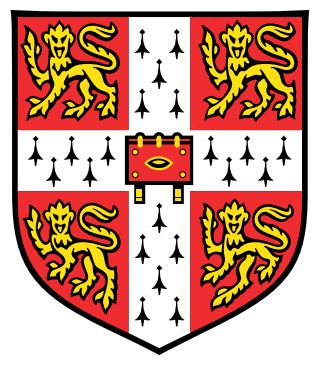

Xue Xiang

Department of Engineering

University of Cambridge

This dissertation is submitted for the degree of

Doctor of Philosophy 

Dedicated to my loving parents. 



\section{Declaration}

I hereby declare that except where specific reference is made to the work of others, the contents of this dissertation are original and have not been submitted in whole or in part for consideration for any other degree or qualification in this, or any other university. This dissertation is my own work and contains nothing which is the outcome of work done in collaboration with others, except as specified in the text and Acknowledgements. This dissertation contains fewer than 65,000 words including appendices, bibliography, footnotes, tables and equations and has fewer than 150 figures.

Xue Xiang

December 2018 



\section{Acknowledgements}

First and foremost, I would like to thank my supervisor, Professor Holger Babinsky, for his invaluable insight and support throughout. I am also grateful to Dr Todd Davidson and Dr Andrea Coschignano, for their great patience in introducing me to the equipment and techniques used in the experiments.

I would like to extend my thanks to the other members of the research group, including Simon Corkery, Charlotte Coles, Isabel Vallina Garcia, Kshitij Sabnis, Pascal Gehlert and Alex Parfett, who have provided many helpful discussions and made my time in Cambridge memorable. My sincere gratitude also goes to the Aero Lab technicians, Dave Martin, Sam Flint and Tony Luckett, for the expert help in running and maintaining the supersonic wind tunnel.

I must acknowledge Cambridge Trust and my benefactor Mrs Catherine, for funding this work, as well as for giving me the opportunity to pursue a doctorate degree. Finally, I would like to take this opportunity to thank my family. Without their love and support, none of this would have been possible. 



\begin{abstract}
In a rectangular cross-section wind tunnel a separated oblique shock reflection is set to interact with the turbulent boundary layer (oblique SBLI) both on the bottom wall and in the corner formed by the intersection of the floor with the side-walls. In such a scenario, shock-induced separation is often seen in each of the streamwise corners, resulting in a highly three-dimensional flow field in the near-wall region.

To examine how the corner separations can affect the 'quasi-two-dimensional' main interaction and by what mechanism this is achieved, an experimental investigation has been conducted. This examines how modifications to the corner separation influence an oblique shock reflection. The nature of the flow field is studied using flow visualisation, Pressure Sensitive Paint and Laser Doppler Anemometry. A nominal freestream Mach number of 2.5 is used for all experiments with a unit Reynolds number of $40 \times 10^{6} \mathrm{~m}^{-1}$, and the shockgenerator angle is set to $8^{\circ}$. The flow conditions are chosen to result in substantial separations both in the corners and along the centreline for the baseline case, which is thought to be a good starting point for this study.

The results show that the size and shape of central separation vary considerably when the onset and magnitude of corner separation change. The primary mechanism coupling these separated regions appears to be the generation of compression waves and expansion fans as a result of the displacement effect of the corner separation. The presence and strength of the expansion waves have been overlooked in previous studies. This is shown to modify the three-dimensional shock-structure and alter the adverse pressure gradient experienced by the tunnel floor boundary layer. It is suggested that a typical oblique SBLI in rectangular channels features several zones depending on the relative position of the corner waves and the main interaction domain. In particular, it has been shown that the position of the corner 'shock' crossing point, found by approximating the corner compression waves by a straight line, is a critical factor determining the main separation size and shape.
\end{abstract}


Thus, corner effects can substantially modify the central separation. This can cause significant growth or contraction of the separation length measured along the symmetry line from the nominally two-dimensional baseline value, giving a fivefold increase from the smallest to the largest observed value. Moreover, the shape and flow topology of the centreline separation bubble is also considerably changed by varying corner effects. 


\section{Table of contents}

$\begin{array}{ll}\text { List of figures } & \text { xv }\end{array}$

$\begin{array}{ll}\text { List of tables } & \text { xxiii }\end{array}$

$\begin{array}{ll}\text { Nomenclature } & \mathbf{x x v}\end{array}$

1 Introduction 1

2 Literature review $\quad 5$

2.1 Shock wave/boundary layer interactions . . . . . . . . . . . 5

2.1.1 Physical introduction . . . . . . . . . . . . . 5

2.1.2 Scaling factors of a turbulent boundary layer . . . . . . . 6

2.1 .3 Normal SBLIs . . . . . . . . . . . . . . . . 7

2.1 .4 Oblique SBLIs . . . . . . . . . . . . . . . . . 12

2.2 Parameters governing the shock-induced separation . . . . . . . . . . 16

2.2.1 Prediction of incipient separation . . . . . . . . . . . 17

2.2.2 Importance of adverse pressure gradient . . . . . . . . . . . . 19

2.3 Corner effects in rectangular channels . . . . . . . . . . . . . . 21

2.3.1 Supersonic corner flows . . . . . . . . . . . . . 21

2.3.2 Corner effects for normal SBLIs . . . . . . . . . . . . 22

2.3.3 Corner effects for oblique SBLIs . . . . . . . . . . . . . 26

2.3.4 Three-dimensional separation flow topologies . . . . . . . . . . . 29

2.4 Flow control . . . . . . . . . . . . . . . . . . . . . . . . 31

2.4 .1 Boundary layer suction . . . . . . . . . . . . . 31

2.4 .2 Vortex generators . . . . . . . . . . . . . . . 33

2.5 Summary and research aims . . . . . . . . . . . . . . 36

2.5.1 Key remaining questions in corner effects for oblique SBLIs . . . . 36

2.5.2 Research aims and approach . . . . . . . . . . . . 37 
3 Experimental facility and techniques

3.1 CUED No.1 supersonic wind tunnel . . . . . . . . . . . . . . . . . 39

3.1 .1 Set-up . . . . . . . . . . . . . . . . . 40

3.2 Corner flow manipulation . . . . . . . . . . . . . . . 42

3.2.1 Corner-control modification . . . . . . . . . . . . . 42

3.2.2 Corner-block modification . . . . . . . . . . . . . . 46

3.3 Flow visualisation . . . . . . . . . . . . . . . . . . . 47

3.3.1 Schlieren imaging . . . . . . . . . . . . . . . 47

3.3.2 Oil-flow visualisation . . . . . . . . . . . . . . . . . . 49

3.4 Pressure measurements . . . . . . . . . . . . . . . . . 50

3.4.1 Static wall pressure taps . . . . . . . . . . . . 50 50

3.4 .2 Pressure sensitive paint . . . . . . . . . . . . . . 52

3.5 Laser Doppler anemometry . . . . . . . . . . . . . . . . . . . . . 57

3.5 .1 General theory . . . . . . . . . . . . . . 57

3.5 .2 Current set-up . . . . . . . . . . . . . . 58

3.5 .3 Flow seeding . . . . . . . . . . . . . . 62

3.5.4 Fitting boundary layer profiles . . . . . . . . . . . . . . 64

3.5.5 Uncertainty in LDA measurements . . . . . . . . . . . . . . . 67

3.6 Experimental errors . . . . . . . . . . . . . . . . . 70

4 Experimental investigation $\quad \mathbf{7 1}$

4.1 Baseline flow . . . . . . . . . . . . . . . . . 71

4.2 Interaction with reduced corner separations $\ldots \ldots \ldots$. . . . . . . 80

4.2.1 Interaction in the presence of corner suction . . . . . . . . . 80

4.2.2 Interaction in the presence of micro-VGs within the corners . . . . 84

4.2.3 Interaction with combined micro-VGs and corner suction . . . . . . 86

4.3 Interaction with increased corner separations . . . . . . . . . . . . . . . 89

4.3.1 Interaction in the presence of small block . . . . . . . . . . . 990

4.3.2 Interaction in the presence of large block . . . . . . . . . . . . 92

4.4 Effects of corner separation on flow field . . . . . . . . . . . . . . . 105

4.4.1 Flow behaviour . . . . . . . . . . . . . . . . . . 105

4.4.2 The factor influencing corner effects . . . . . . . . . . . . 106

5 Physical mechanism of corner effects 109

5.1 Influence of corner waves . . . . . . . . . . . . . . . . . . . . 109

5.2 Three-dimensional oblique SBLI structure . . . . . . . . . . . . . . 110

5.2.1 Corner effects on 'quasi-two-dimensional' interactions . . . . . . . 110 
5.2.2 Corner effects on interactions with increased separation length . . . 112

5.2.3 Corner effects on interactions with reduced separation length . . . . 114

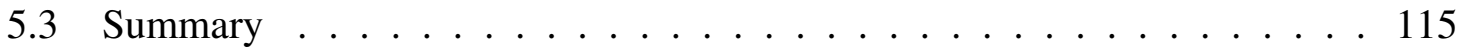

6 Conclusions and future work $\quad 117$

6.1 Conclusions . . . . . . . . . . . . . . . . . . . 117

6.2 Recommendations and future work . . . . . . . . . . . . . . . . . . 119

$\begin{array}{ll}\text { References } & 121\end{array}$ 



\section{List of figures}

1.1 Examples of rectangular supersonic mixed-compression inlets: (a) XB-70 Valkyrie; (b) Concorde; (c) schematic of the inlet flow field, adapted from [110]. 2

2.1 Illustration of SBLI classification based on the flow condition downstream of the interaction: $(a)$ transonic; $(b)$ supersonic. . . . . . . . . . . . . 6

2.2 The flow field of an attached normal SBLI: $(a)$ sketch of the interaction structure; $(b)$ wall-normal stagnation pressure distribution; $(c)$ streamwise wall pressure distribution, adapted from [7]. . . . . . . . . . . . . 8

2.3 Variation of boundary layer parameters through an attached normal SBLI, subscript 0 refers to values upstream of the interaction, from [42]. . . . . .

2.4 The flow field of a separated normal SBLI: $(a)$ sketch of the interaction structure; $(b)$ wall-normal stagnation pressure distribution; (c) streamwise wall pressure distribution, adapted from Seddon [112]. . . . . . . . . . . 10

2.5 Variation of boundary layer parameters through a separated normal SBLI, from [42]. . . . . . . . . . . . . . . . . . . . .

2.6 Schlieren photograph of an attached oblique shock reflection, adapted from Dèlery [7]. . . . . . . . . . . . . . . . . . . . . . . . . . . . . 12

2.7 The flow field of an attached oblique SBLI: $(a)$ sketch of the interaction structure, reproduced by Giepman [60] following Délery and Bur [40]; (b) streamwise wall pressure distribution. . . . . . . . . . . . .

2.8 Schlieren photograph of a separated oblique shock reflection, adapted from Dèlery [7]. . . . . . . . . . . . . . . . . . . . . . . . . . . . . . . . . . . . 14

2.9 The flow field of a separated oblique SBLI: $(a)$ sketch of the interaction structure; $(b)$ streamwise wall pressure distribution, based on the work of Délery and Bur $[40] . \ldots \ldots \ldots \ldots \ldots \ldots$ 
2.10 Computations of wall pressure distribution for oblique shock reflection in $M_{0}=2$ flow. The incident shock strength increases from condition 1 to 5, with symbols denoted $(\boldsymbol{\nabla})$ separation point and $(\square)$ impingement location of the first expansion wave from the downstream wedge corner, adapted from Matheis and Hickel [85]. . . . . . . . . . . . . . . . . . 16

2.11 The onset of normal shock-induced separation, from [42] . . . . . . . . . . 17

2.12 The onset of shock-induced separation in transonic channel flows, from Bruce et al. [25]: (a) correlation with $R_{e \theta}$, following Sajben et al. [109]; (b) improved parameter $\delta_{i}^{*} / W$ accounting for confined effects. Open symbols represent attached central flow, filled symbols for separated central flow.

2.13 Wall pressure $\left(p_{w}\right)$ and wall friction $\left(c_{f}\right)$ distribution measured by Smith and Smits [118] for two compression ramp arrangements: $(a)$ model $\mathrm{A}$ as a sharp corner; $(b)$ model B as a rounded corner. The beginning and end of the concave and convex curvatures are marked on the abscissa with vertical lines. 19

2.14 Corner vortices of supersonic turbulent flow in a square duct, adapted from Davis and Gessner [38]. $a$ represents duct half width. . . . . . . . . .

2.15 Oil-flow visualisation for normal SBLIs in supersonic wind tunnel facilities with rectangular cross-sections: ( $a$ ) $M_{\infty}=1.5$ by Burton et al. [29]; (b) $M_{\infty}=1.45$ by Doerffer and Szwaba [48]; (b) $M_{\infty}=1.59$ by Chriss et al. [32]. Flow from left to right. . . . . . . . . . . . . . .

2.16 Oil-flow patterns on the tunnel floor for a $M_{\infty}=1.4$ shock, measured by Bruce et al. [25]: ( $a$ ) baseline flow; $(b)$ corner-suction applied. . . . . . .

2.17 Oil-flow patterns on the tunnel floor for a $M_{\infty}=1.5$ shock, measured by Bruce et al. [25]: (a) baseline flow; $(b)$ corner-VGs applied. . . . . . . .

2.18 Three-dimensional diagram of normal SBLI in a corner region, proposed by Burton and Babinsky [28]. . . . . . . . . . . . . . . . .

2.19 Schematic three-dimensional flow field for an oblique SBLI in a rectangular duct, from Eagle and Driscoll [51] . . . . . . . . . . . . . . .

2.20 Corner effects for a separated oblique shock reflection, adapted from Babinsky et al. [10]: (a) schematic diagram of the corner shock wave; $(b)$ proposed variation of separation lengths along the tunnel floor centreline as a function of viscous aspect ratio.

2.21 Simulated oil-flow visualisation on the tunnel floor for a strong interaction at $M_{\infty}=2.9$ in a small-aspect-ratio channel of $w / h=0.25$ : (a) without the QCR terms; $(b)$ with the QCR terms. Blue shaded region indicates separation, from [12]. 
2.22 Three-dimensional flow fields associated with flow separation described by Perry and Hornung [104]: (a) owl-face of the first kind; $(b)$ owl-face of the second kind, adapted from Colliss [34] . . . . . . . . . . . . 30

2.23 Schematic representation of the flow in a separated bubble with suction applied, from Délery and Bur [40].

2.24 Types of micro-vortex generators, adapted from [81]: ( $a$ ) forwards wedge; $(b)$ backwards wedge; $(c)$ counter-rotating vanes; $(d)$ single rotating vane. Height $\mathrm{h}$ is the key parameter. . . . . . . . . . . . . . . . . . . 33

2.25 Variation of non-dimensional vortex strength with: $(a)$ effective height $h_{e f f}^{+}$, from Nolan [97]; (b) downstream distance, from Ashill et al. [5]. . . . . . .

3.1 CUED No.1 Supersonic wind tunnel: $(a)$ schematic illustration; $(b)$ photograph of the tunnel without doors and nozzles, adapted from [37]. . . . . . 39

3.2 Setup for the working section. . . . . . . . . . . . . . . . . . 41

3.3 Experimental arrangements of corner flow control. . . . . . . . . . . . . 42

3.4 Suction plate diagram of corner suction (dimensions in $\mathrm{mm}$ ) . . . . . . . . . 43

3.5 Schematic diagram of flow pattern through a quarter-circle orifice plate. . . 43

3.6 Dimensions of corner vane (in mm) . . . . . . . . . . . . . . . . 45

3.7 Experimental arrangements of corner obstacles. . . . . . . . . . . . . . . 46

3.8 Schlieren visualisation technique: (a) 'z-type' optical set-up; $(b)$ typical result. 47

3.9 Set-up for static wall pressure measurement, modified from [99]. . . . . . . 50

3.10 Hole geometry error on the pressure measured by static tap as a function of the Reynolds number based on hole diameter and friction velocity, reproduced from [117] . . . . . . . . . . . . . . . . . . . 51

3.11 Pressure sensitive paint technique: (a) underlying physics; $(b)$ experimental set-up, adapted from [34]. . . . . . . . . . . . . . . . . . . . .

3.12 PSP processing procedure: $(a)$ 'wind-off' image, $I_{r e f} ;(b)$ 'wind-on' image, $I ;(c)$ ratio of the two, $I_{r e f} / I$, tap locations shown by the squares. . . . . . .

3.13 PSP calibration: (a) calibration curve relating luminescence intensity and pressure; $(b)$ map of the global pressure field. . . . . . . . . . . . . . 54

3.14 Comparison of PSP data and pressure taps along the tunnel floor centreline. 55

3.15 Laser Doppler anemometry technique: $(a)$ schematic of interference pattern in beam overlap volume; $(b)$ scattered light intensity variation due to a particle passing through the measurement volume, known as Doppler burst signal; $(c)$ relationship between velocity and Doppler frequency. [37] . . . 
3.16 Set-up of Laser Doppler anemometer: (a) layout of the forward-mode equipment mounted on a 3-axis traverse, adapted from [35]; (b) arrangement of the emitting head, shifted beams on the downstream side. . . . . . . . . .

3.17 Example light patterns of laser alignment: (a) patterns through a $35 \mu \mathrm{m}$ pin-hole, interference fringes can be seen when aligned; $(b)$ floor-finding technique, even distribution around the alignment target indicates that the measurement volume is at the floor. [34] . . . . . . . . . . . . . .

3.18 Schematic of the seeding particle generator, with the path of paraffin droplets indicated by the red lines, adapted from [34].

3.19 Distribution of LDV data rate on the central symmetry plane: (a) streamwise data rates taken at $y=15 \mathrm{~mm} ;(b)$ boundary layer seeding rates and velocity data taken at $x=160 \mathrm{~mm} \ldots \ldots \ldots \ldots \ldots \ldots$

3.20 Examples of analytical profiles fitted to discrete data in logarithmic wall units: $(a)$ upstream of oblique SBLI, at $x=80 \mathrm{~mm} ;(b)$ downstream of the interaction, at $x=160 \mathrm{~mm}$.

3.21 Errors in calculated integral boundary layer parameters due to discretization: (a) variation of errors against measurement resolution; $(b)$ critical measurement resolution required to give better than $5 \%$ error in all integral parameters; $(c)$ error caused by misalignment of the floor, from Titchener et al. [131]. . . . . . . . . . . . . . . . . . . . . .

4.1 Schlieren photograph for the baseline flow: $M=2.5$, wedge angle $=8^{\circ} \ldots .72$

4.2 Variation in streamwise velocity at $y=15 \mathrm{~mm}, z=0 \mathrm{~mm}$ for the baseline flow. 72

4.3 Boundary-layer profiles measured with LDA along the tunnel floor centreline for the baseline case: $(a)$ inflow boundary layer profile at $x=80 \mathrm{~mm} ;(b)$ inflow boundary layer profile in law-of-the-wall coordinates; $(c)$ downstream boundary layer profile at $x=160 \mathrm{~mm} ;(d)$ downstream boundary layer profile in law-of-the-wall coordinates. . . . . . . . . . . . . . . . . 73

4.4 Oil-flow visualisation on the tunnel floor for the baseline flow. . . . . . . . 74

4.5 Interpretation of flow topology on the tunnel floor for the baseline flow by $(a)$ surface oil-flow visualisation; $(b)$ schematic illustration of separation sizes extracted from the oil flow. Limiting streamlines and the footprint of the separation are suggested for clarity; the vertical line indicates the inviscid shock location. . . . . . . . . . . . . . . . . . . . . . . . 74

$4.6 \quad P / P_{0}$ on the tunnel floor for the baseline flow. . . . . . . . . 75

4.7 Static wall pressure distribution along the tunnel floor centreline for the baseline flow. . . . . . . . . . . . . . . . . . . 76 
4.8 Oil-flow visualisation in the right sidewall corner for the baseline flow. . . . 76

4.9 Structure of the wave pattern induced by the corner separation illustrated by: (a) PSP map on the right sidewall with extent of corner separation shown by a dotted line border; $(b)$ static wall pressure distribution measured by pressure tappings at $z=57 \mathrm{~mm}, y=20 \mathrm{~mm} . \ldots \ldots \ldots$

4.10 Illustration of corner waves on baseline flow: (a) schematic diagram of corner wave structure; $(b)$ corner shock footprint indicated on oil-flow image. 79

4.11 Schlieren photographs with corner suction applied at: (a) $x_{\text {slot }}=20 \mathrm{~mm} ;(b)$ $x_{\text {slot }}=100 \mathrm{~mm} ;(c) x_{\text {slot }}=150 \mathrm{~mm} \ldots \ldots \ldots \ldots$

4.12 Surface flow visualisation on the tunnel floor for the flow controlled by corner suction at $x_{\text {slot }}=100 \mathrm{~mm}$ : $(a)$ oil-flow image; $(b)$ PSP map. . . . . . . . . .

4.13 Oil-flow visualisation on the tunnel floor for the flow controlled by corner suction at $x_{\text {slot }}=150 \mathrm{~mm}$.

4.14 Oil-flow visualisation on the tunnel floor for the flow controlled by corner suction at $x_{\text {slot }}=20 \mathrm{~mm}$.

4.15 Schlieren photograph with micro-VGs applied at $x_{v g}=50 \mathrm{~mm}$.

4.16 Oil-flow visualisation on the tunnel floor for the flow controlled by microVGs at $x_{v g}=50 \mathrm{~mm}$.

4.17 Schematic illustration for flow control mechanism of micro-VGs within the corner: $(a)$ vortices produced by the micro-vane $(b)$ streamwise velocity contour downstream of VG. . . . . . . . . . . . . . . . . . .

4.18 Schlieren photograph with micro-VGs $\left(x_{v g}=50 \mathrm{~mm}\right)$ and corner suction $\left(x_{\text {slot }}=100 \mathrm{~mm}\right)$ employed in tandem. . . . . . . . . . .

4.19 Surface flow visualisation on the tunnel floor for the flow with combined control applied: (a) oil-flow image; $(b)$ PSP map. . . . . . . . . . . . . .

4.20 Variation in streamwise velocity at $y=15 \mathrm{~mm}, z=0 \mathrm{~mm}$ for flows with reduced corner separations. . . . . . . . . . . . . . . . . . . . . 89

4.21 Schlieren photograph with small corner obstacle introduced at $x_{\text {block }}=105 \mathrm{~mm} .90$

4.22 Oil-flow visualisation on the tunnel floor for the flow disturbed by small corner blocks at $x_{\text {block }}=105 \mathrm{~mm} \ldots \ldots \ldots$. . . . . . . . . 90

$4.23 P / P_{0}$ for the tunnel floor with small blocks at $x_{\text {block }}=105 \mathrm{~mm} . \quad \ldots . . . \quad$. 91

4.24 Static wall pressure distribution on the tunnel floor centreline for small corner-block case. . . . . . . . . . . . . . . . . .

4.25 Schlieren photographs with large corner obstacle introduced across the interaction domain: $(a) x_{\text {block }}=120 \mathrm{~mm} ;(b) x_{\text {block }}=130 \mathrm{~mm} . \ldots \ldots$. . . . 
4.26 Schlieren photographs with large corner obstacle introduced ahead of the incident shock: $(a) x_{\text {block }}=75 \mathrm{~mm} ;(b) x_{\text {block }}=85 \mathrm{~mm}$.

4.27 Surface oil-flow visualisation on the tunnel floor for the oblique SBLIs with large corner blocks: $($ a $) x_{\text {block }}=130 \mathrm{~mm} ;(b) x_{\text {block }}=125 \mathrm{~mm} ;(c) x_{\text {block }}=$ $120 \mathrm{~mm} ;(d) x_{\text {block }}=115 \mathrm{~mm} ;\left(\right.$ e) $x_{\text {block }}=105 \mathrm{~mm} ;(f) x_{\text {block }}=95 \mathrm{~mm} ;(\mathrm{g})$ $x_{\text {block }}=90 \mathrm{~mm} ;(h) x_{\text {block }}=85 \mathrm{~mm}$; (i) $x_{\text {block }}=75 \mathrm{~mm}$. The approximate footprint of the corner shock is indicated by red lines. . . . . . . . . . . .

4.28 Oil-flow visualisation on the tunnel floor with the central separation region of baseline interaction overlaid : (a) $x_{\text {block }}=120 \mathrm{~mm}$; $(b) x_{\text {block }}=90 \mathrm{~mm}$. . .

4.29 Surface flow topology of the centreline separation underneath the oblique SBLIs when the crossing point of corner shocks locates inside the separated region: (a) crossing point downstream to the separation onset of baseline flow, from $x_{\text {block }}=130 \mathrm{~mm}$ to $x_{\text {block }}=95 \mathrm{~mm} ;(b)$ crossing point at the separation onset of baseline flow, for $x_{b l o c k}=90 \mathrm{~mm} . \ldots \ldots \ldots$

4.30 Oil-flow visualisation on the tunnel floor for $x_{\text {block }}=75 \mathrm{~mm}$ with the central separation region of baseline interaction overlaid.

4.31 Surface flow topology of the centreline separation underneath the oblique SBLIs when the crossing point of corner shocks locates ahead of the separated region, for $x_{\text {block }}=85 \mathrm{~mm}$ and $x_{\text {block }}=75 \mathrm{~mm}$. . . . . . . . . . . . . 99

$4.32 P / P_{0}$ for the tunnel floor for $x_{\text {block }}=120 \mathrm{~mm}$ with increased $L_{\text {sep }} \ldots \ldots$. . . 100

$4.33 P / P_{0}$ for the tunnel floor for the oblique SBLIs with reduced $L_{\text {sep }}$ : (a) $x_{\text {block }}=85 \mathrm{~mm} ;(b) x_{\text {block }}=75 \mathrm{~mm}$. The approximate footprint of the corner shock is indicated by red lines. . . . . . . . . . . . . . . . . . 100

4.34 Static wall pressure distribution on the tunnel floor centreline. . . . . . . . 101

4.35 Boundary layer velocity distribution at $x=80 \mathrm{~mm}$ for the tunnel floor centreline. 101

4.36 Boundary layer velocity distribution at $x=160 \mathrm{~mm}$ for the tunnel floor centreline. . . . . . . . . . . . . . . . . . . . 102

4.37 Variation in streamwise velocity at $y=15 \mathrm{~mm}, z=0 \mathrm{~mm}$ for flows with increased corner separations. . . . . . . . . . . . . . . . . 103

4.38 Schlieren photographs with large corner obstacle applied at $x_{\text {block }}=85 \mathrm{~mm}$ : (a) elongated blocks of $L_{\text {block }}=40 \mathrm{~mm} ;(b)$ standard blocks of $L_{\text {block }}=20 \mathrm{~mm} .104$

4.39 Surface oil-flow visualisation on the tunnel floor with $L_{\text {block }}=40 \mathrm{~mm}$ blocks applied at $x_{\text {block }}=85 \mathrm{~mm} \ldots \ldots \ldots \ldots$. . . . . . . . . . . 104

4.40 Boundary layer velocity distribution for different block lengths $L_{b l o c k}$ along the tunnel floor centreline: $(a)$ at $x=80 \mathrm{~mm} ;(b)$ at $x=160 \mathrm{~mm}$. . . . . . . 105 
4.41 Variation of the scaled centreline separation length $L_{\text {sep }} / \delta^{*}$ as a function of streamwise position of corner shocks intersection point. . . . . . . . . . 107

4.42 Variation of the scaled centreline separation width $L_{f} / w$ as a function of streamwise position of corner shocks intersection point. . . . . . . . . 108

5.1 Schematic illustration of corner effects on 'quasi-two-dimensional' interaction: (a) separation zones on floor; $(b)$ diagram of SBLI structure on the central symmetry plane. . . . . . . . . . . . . . . . . . 111

5.2 Schematic illustration of corner effects on interactions with increased $L_{\text {sep }}$ : (a) separation zones on floor; $(b)$ diagram of SBLI structure on the central symmetry plane. . . . . . . . . . . . . . . . . . . 113

5.3 Schematic illustration of corner effects on interactions with reduced $L_{\text {sep }}$ : (a) separation zones on floor; $(b)$ diagram of SBLI structure on the central symmetry plane. . . . . . . . . . . . . . . . . . . . . . 114 



\section{List of tables}

3.1 Suction strength quantities for corner-bleed tests. . . . . . . . . . . . . . 44

3.2 Details of the LDA configuration. . . . . . . . . . . . . . 60

3.3 Errors in the variables determined from experimental measurements. . . . . 70

4.1 Summary for the control effectiveness of corner-bleed (in $\mathrm{mm}$ ). . . . . . . . 83

4.2 Summary of separation-related parameters for oblique SBLIs with reduced corner separations (in mm) . . . . . . . . . . . . . . . . 88

4.3 Boundary layer parameters at $x=80 \mathrm{~mm}$ along the tunnel floor centreline for baseline and experiments with reduced corner separations. . . . . . . . 88

4.4 Boundary layer parameters at $x=160 \mathrm{~mm}$ along the tunnel floor centreline for baseline and experiments with reduced corner separations. . . . . . . . 88

4.5 Centre separation parameters for oblique SBLIs with increased corner separations $($ in $\mathrm{mm}) \ldots \ldots \ldots$. . . . . . . . . . . . . . . . . . 93

4.6 Boundary layer parameters at $x=80 \mathrm{~mm}$ along the tunnel floor centreline for experiments with increased corner separations. . . . . . . . . . . . 102

4.7 Boundary layer parameters at $x=160 \mathrm{~mm}$ along the tunnel floor centreline for experiments with increased corner separations. . . . . . . . . . . . . 102 



\section{Nomenclature}

\section{Roman Symbols}

$\begin{array}{ll}A & \text { area } \\ B & \text { log-law constant, taken as } 5 \\ C_{D i} & \text { orifice plate discharge coefficient } \\ C_{f} & \text { skin friction coefficient } \\ C_{p} & \text { pressure coefficient } \\ c_{p} & \text { constant pressure specific heat capacity } \\ d_{f} & \text { distance between fringes in LDA measurement volume } \\ d_{p} & \text { LDA seeding particle diameter } \\ f_{D} & \text { Doppler frequency } \\ H & \text { compressible shape factor } \\ H_{i} & \text { incompressible shape factor } \\ h & \text { height of vortex generators } \\ L_{s e p} & \text { length scale } \\ L_{f} & \text { upstream influence length } \\ L_{0} & \text { spanwise extent of centreline separation } \\ & \end{array}$




\begin{tabular}{|c|c|}
\hline$\dot{m}$ & mass flow rate \\
\hline$n$ & air refractive index \\
\hline$N_{\varepsilon}$ & measurement resolution giving error of $\varepsilon \%$ \\
\hline$P$ & pressure \\
\hline$R^{2}$ & coefficient of determination \\
\hline$R_{e \delta}$ & Reynolds number based on boundary layer thickness \\
\hline$R_{e \theta}$ & Reynolds number based on boundary layer momentum thickness \\
\hline$S_{k}$ & Stokes number \\
\hline$s$ & Musker model fitting parameter, taken as 0.01093 \\
\hline$T$ & temperature \\
\hline$t$ & time \\
\hline$U, u$ & streamwise velocity \\
\hline$u_{\tau}$ & friction velocity \\
\hline$w$ & wind tunnel width \\
\hline$x$ & streamwise coordinate \\
\hline$x_{\text {slot }}$ & streamwise position of the leading edge of slot \\
\hline$x_{v g}$ & streamwise position of the trailing edge of micro-vortex generator \\
\hline$y$ & floor-normal coordinate \\
\hline$\triangle y$ & misalignment of tunnel floor in LDA traverse \\
\hline$y_{s}$ & height of sonic line in boundary layer \\
\hline & spanwise coordinate \\
\hline
\end{tabular}

\section{Greek Symbols}

LDA emitting head pitch angle

ratio of specific heats 


\begin{tabular}{|c|c|}
\hline$\delta$ & boundary layer thickness \\
\hline$\delta_{c}^{*}$ & compressible displacement thickness \\
\hline$\delta_{i}^{*}$ & incompressible displacement thickness \\
\hline$\varepsilon$ & deflection angle of light \\
\hline$\theta_{c}$ & compressible momentum thickness \\
\hline$\theta_{i}$ & incompressible momentum thickness \\
\hline$\kappa$ & log-law coefficient, taken as 0.41 \\
\hline$\lambda$ & wavelength \\
\hline$\mu$ & dynamic viscosity \\
\hline$v$ & kinematic viscosity \\
\hline$\phi$ & half-angle between LDA beam pair \\
\hline$\Psi$ & LDA emitting head rotation \\
\hline$\rho$ & density \\
\hline$\Sigma$ & van Driest compressibility transform scaling parameter \\
\hline$\sigma$ & standard deviation \\
\hline$\tau$ & shear stress \\
\hline
\end{tabular}

\section{Superscripts}

fluctuation value
$+\quad$ non-dimensional variable in wall units

\section{Subscripts}

0

$\infty$

$e$

w stagnation condition or initial value

free-stream condition

value at boundary layer edge

value at wall 


\section{Acronyms / Abbreviations}

CFD computational fluid dynamics

CUED Cambridge University Engineering Department

DNS direct numerical simulations

FSA frequency/burst spectrum analyser for LDA

LDA laser Doppler anemometry

LES large eddy simulation

RANS Reynolds averaged Navier-Stokes

NASA National Aeronautics and Space Administration

PDM photo detector/multiplier

PIV particle image velocimetry

PSP pressure sensitive paint

SBLI shock wave/boundary layer interaction

VG vortex generator 


\section{Chapter 1}

\section{Introduction}

Over the past decades air transportation has risen from a niche market to a world leading industry. In 2017 the commercial aircraft sector has generated a turnover in excess of $£ 254$ billion and the passenger traffic demand is predicted to increase by around $5.1 \%$ annually [82]. This significant growth is primarily driven by the emerging markets in AsiaPacific, the Middle East and the Latin America region. The development of supersonic aircraft for efficient long-range travel has therefore been a strategic focus for top airlines, striving to connect these businesses to the developed western markets and achieve a high volume of air traffic globally [75, 82]. One of the critical technology gaps in the design of such flight vehicles is the requirement of a more effective supersonic mixed-compression inlet. Such engine inlet systems are desirable for aircraft faster than $M=2$, where Pitot intakes suffer from excessive shock losses while external compression intakes approach the limit of their effectiveness [99].

Mixed-compression inlets serve to capture the oncoming supersonic flow and slow it down to subsonic speeds (typically Mach 0.3 to 0.6 ) for the following turbo-machinery, while aiming to conserve as much mechanical energy as possible. As shown in figure 1.1, such engine inlets often take the form of rectangular ducts and achieve deceleration by a number of oblique shock waves, which interact with the boundary layer forming along the intake walls (SBLIs). The high adverse-pressure-gradient (APG) flow field created by such an oblique shock system results in boundary-layer thickening and may eventually trigger flow separation. This shock-induced separation can lead to a significant total pressure loss and flow unsteadiness. In extreme circumstances where the separated region in core flow grows too large the inlet will unstart, resulting in a considerably reduced thrust or even engine shut down [71]. It is therefore of great importance to apply flow control to mitigate or eliminate such flow separation. The successful application of such control techniques is however hindered by a lack of knowledge of the underlying physics of oblique SBLIs. 
(a)

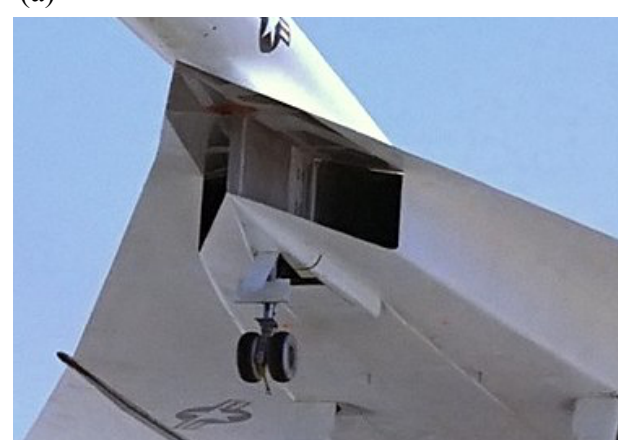

(b)

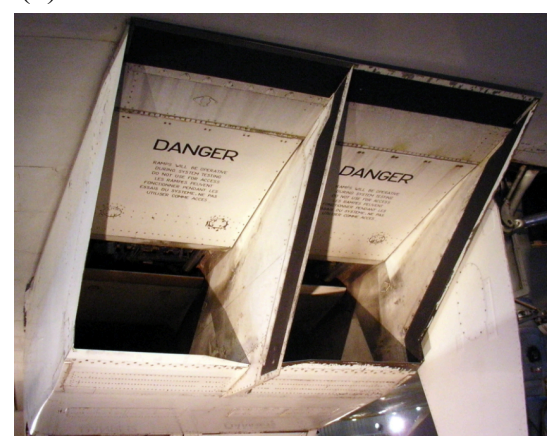

(c)

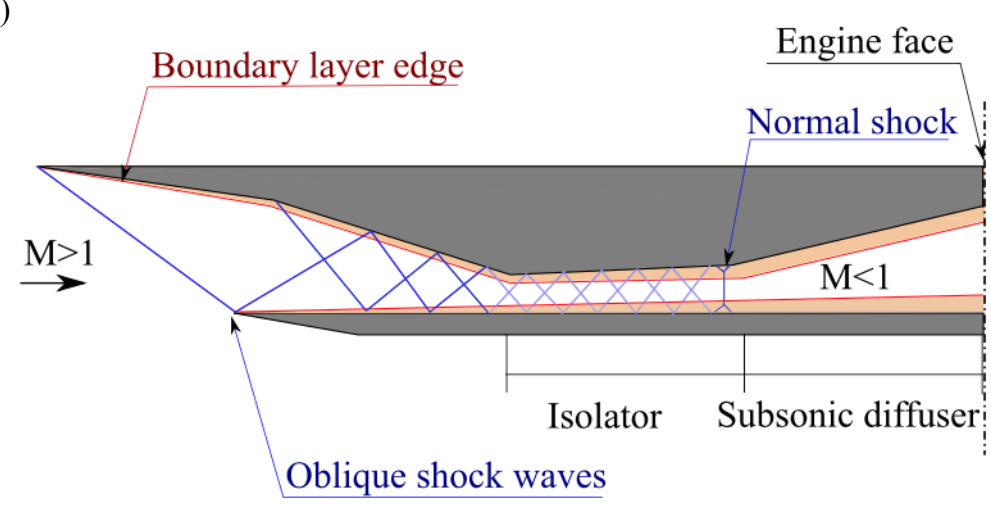

Fig. 1.1 Examples of rectangular supersonic mixed-compression inlets: (a) XB-70 Valkyrie; (b) Concorde; $(c)$ schematic of the inlet flow field, adapted from [110].

Experimental investigations into oblique SBLIs have been mainly conducted in supersonic wind tunnels. The geometric feature shared by such research tools and actual inlets is the rectangular cross-section with streamwise corners. In the corner of a straight channel, where the floor and sidewall boundary layers meet and generate a corner boundary layer, shockinduced separation is more likely to occur or occur further upstream compared to flowfield elsewhere, resulting in a highly three-dimensional flow in the near-wall region.

Many investigations into SBLIs have focused solely on the core flow in the central region, assuming that flow away from the sidewall can be treated as nominally two-dimensional. This assumption is sometimes strengthened by the observation that separated regions far from the corners are seen to be of a relatively two-dimensional appearance. However, more recently it has been noted that the three-dimensionality induced by corner separations is not restricted to the near-wall regions but can influence the flow in the centre. A possible link between separations in the corners and the mid-span has been suggested by some investigations; 
however the mechanisms as to how corner separations transmit their effects to the flow field elsewhere remains unclear.

One way to reduce the detrimental effects of corner flows would be to utilise flow control to reduce or eradicate corner separations. However, to date, no control method has been found to be consistently successful in achieving this for oblique SBLIs. The difficulties in mitigating corner separations have so far hampered attempts to systematically explore the interplay between the local flow and the overall flowfield. This lack of understanding of corner effects also presents a major obstacle for computational fluid mechanics (CFD) to correctly capture the three-dimensional near-wall flow structure. The inadequacy of simulation techniques in turn inhibits an accurate prediction of inlet performance or designs of effective flow control systems.

This experimental study seeks to extend the current state of knowledge of the threedimensional flow structures induced by corner effects and their influence on the flow field elsewhere for an oblique SBLI. Mechanisms explaining any observed interplay between the corner separations and centreline flows will also be explored.

Following a comprehensive review of the physics of shock wave boundary layer interactions, the current understanding of corner effects for such flows is summarised. Chapter 3 describes the experimental facilities and methods used to study this problem. Results from the experiments are presented in Chapter 4; this lays down the foundation for Chapter 5, which proposes physical mechanisms for corner effects drawn from the experimental findings. Finally, Chapter 6 provides the conclusions of this thesis and their implications on future work. 



\section{Chapter 2}

\section{Literature review}

\subsection{Shock wave/boundary layer interactions}

\subsubsection{Physical introduction}

The interaction of a shock wave with a boundary layer (Shock-wave/Boundary-Layer Interaction SBLI) occur in almost all practical transonic, supersonic and hypersonic vehicles [62], and is hence considered one of the fundamental problems of high speed flows in air. SBLIs have been widely studied since the 1940s, often by investigating simplified canonical problems which form the building blocks of more complex flows. Extensive measurements are available for the most common configurations including: normal shock interactions [6, 27], impinging oblique shock interactions [63, 105], over-expanded nozzles [18, 53], swept fin problems [17, 100], blunt fin interactions [26, 132] and compression ramp interactions $[126,140]$. Of these, the transonic interaction (figure 2.1a) and the supersonic incident-reflecting interaction (figure 2.1b) are probably the most important problems. In the transonic interaction (also termed normal SBLI), a normal or near-normal shock decelerates the incoming supersonic flow to a subsonic condition, so that changes in the post-shock flow can feed forward and impose constraints on the shock wave causing the interaction; conversely, the reflecting SBLI is associated with an oblique incident shock and the flow downstream remains to be supersonic, therefore having no impact on the SBLI. Both of these interactions are nominally two-dimensional and past research has generally concentrated on the flow behaviour well away from sidewalls or edge effects (refer to the reviews of classic 2-D SBLI of Adamson and Messiter [2], Délery and Dussauge [45], Babinsky and Harvey [7]).

However, in recent studies $[7,57]$ it has been found that even in nominally two-dimensional/ axisymmetric flows, the mean flow can be three-dimensional. Gaitonde [57] concluded that 

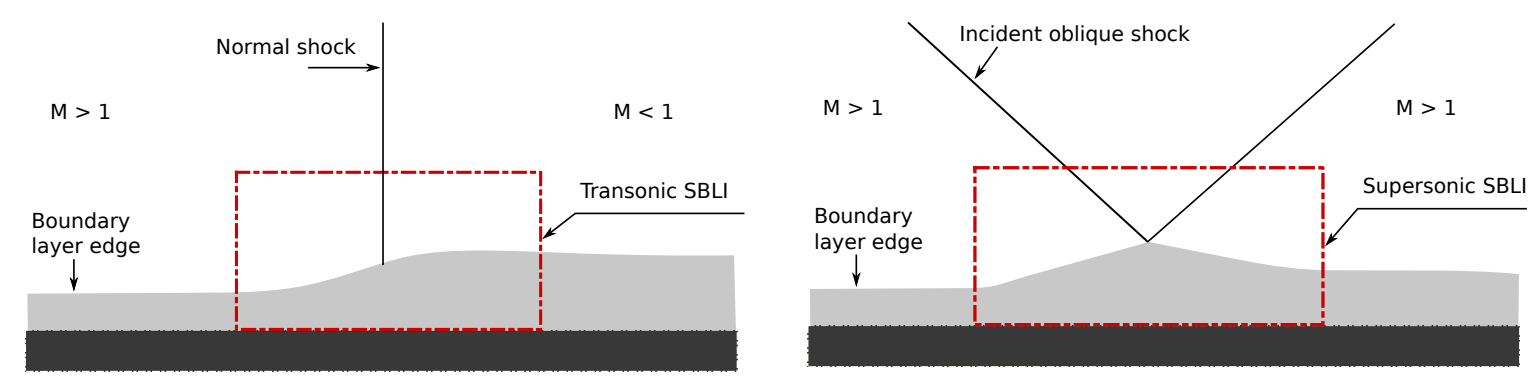

Fig. 2.1 Illustration of SBLI classification based on the flow condition downstream of the interaction: $(a)$ transonic; $(b)$ supersonic.

the unintended three-dimensional effects were frequently caused by experimental constraints, such as the finite size of supersonic wind tunnels. It has also been stated that even with advanced experimental diagnostics (e.g. Laser Doppler anemometry LDA; particle image velocimetry PIV) and numerical schemes (e.g. Reynolds-averaged Navier-Stoks RANS; large eddy simulation LES; direct numerical simulation DNS), three-dimensionality of SBLIs remains to be an area of unanswered questions [7].

The following sections first give an overview of the fundamental two-dimensional SBLIs, both attached and separated, before the factors governing the shock-induced separation being discussed. Attention is then focused on the three-dimensional effects for such flows in rectangular channels, which is the most common geometry for such investigations and therefore the main interest of current study. Methods of controlling shock-induced separations are also examined, allowing an insight into corner flow manipulation.

\subsubsection{Scaling factors of a turbulent boundary layer}

Within the two categories of SBLIs shown in figure 2.1, interactions with a turbulent boundary layer have been the focus of much of the research to date because of their greater practical importance [42]. Hence only the turbulent cases will be discussed here.

A boundary layer can be viewed as a thin viscous region of thickness $\delta$, through which the flow velocity is decelerated from the external free-stream value $u_{e}$ to zero at the wall, where the no-slip condition must be satisfied. In a typical interaction process, the shock wave, carrying an abrupt pressure rise, penetrates into a boundary layer. The response of the boundary layer to this externally-imposed adverse pressure gradient depends considerably on its velocity distribution, which can be characterized by the integral parameters defined in equations (2.1).

The displacement thickness $\delta_{c}^{*}$ describes the deficit of mass relative to the equivalent inviscid flow. In addition, the displacement concept determines the flow deflection along an 
effective surface, which is the body shape augmented by $\delta_{c}^{*}$. The momentum loss caused by the presence of the boundary layer, which results in a drag increase or an efficiency loss, is represented by the momentum thickness $\theta_{c}$. The ratio of these two, designated as the shape factor $\mathrm{H}$, is of significant importance as it characterises the level of mean kinetic energy of the velocity profile:

$$
\delta_{c}^{*}=\int_{0}^{\delta}\left(1-\frac{\rho u}{\rho_{e} u_{e}}\right) d y \quad ; \quad \theta_{c}=\int_{0}^{\delta} \frac{\rho u}{\rho_{e} u_{e}}\left(1-\frac{u}{u_{e}}\right) d y \quad ; \quad H=\frac{\delta_{c}^{*}}{\theta_{c}}
$$

The higher the energy level (corresponding to a 'fuller' streamwise-velocity profile), the lower the value of the shape factor. However, the shape factor computed with the compressible integral thicknesses is a strong function of $\mathrm{M}$, and is therefore less practical as a universal parameter [7]. Simplified versions of equations (2.1), in which constant density across the boundary layer (i.e. $\rho=\rho_{e}$ ) is assumed, are hence used in preference. Such an assumption has been validated by the study of Morkovin [95], which states that compressibility tends to have little to no impact on the boundary-layer behaviour until at least $M=3$. This benefit, combined with the difficulty of accurately determining the density variation from experiments, is the reason why the analysis presented in this thesis is restricted to the incompressible integral boundary layer parameters $\delta_{i}^{*}, \theta_{i}, H_{i}$ (although often referred to simply as the boundary layer parameters from here on).

Another critical dimension to be considered is $y_{s}$, the position of the sonic point on the boundary layer profile. This height defines the subsonic region within the incoming boundary layer, which enables the pressure rise imparted by the shocks to be communicated upstream. For an adiabatic wall condition, as $H_{i}$ increases, the subsonic layer experiences a rapid thickening [42].

\subsubsection{Normal SBLIs}

Although the current study focuses on supersonic SBLIs, the physics of normal shock interaction is also discussed because of the (obvious) similarities between these two types of interactions [7]. Also, in transonic SBLIs previous studies have found some mechanisms for corner effects which may also play a role in supersonic SBLIs (will be detailed in section 2.3). Knowledge on normal SBLIs hence provides useful correlations for understanding the more complex flow fields generated by an oblique interaction.

Although normal shocks exist at all $\mathrm{M}$, normal SBLIs are mainly observed at moderate supersonic speeds with $M$ below 2 [7]. Such interactions can be further split into two 
subgroups: attached SBLIs with $M \lesssim 1.3$ typically [1, 32, 111]; and separated SBLIs with shocks strong enough to separate the boundary layer.

\section{Attached interaction}

The basic attached SBLI structure of a weak normal shock interacting with the boundary layer on a flat plate is sketched in figure 2.2a. The incoming flow is supersonic except for the thin subsonic layer in contact with the wall. 'Feeling' the presence of the shock, the subsonic region thickens to communicate the pressure rise information upstream. This results in a significant displacement of the boundary layer edge ahead of the incident shock location. The supersonic flow must therefore be deflected away from the surface, which is achieved by a series of compression waves extending from the sonic line. These compression waves

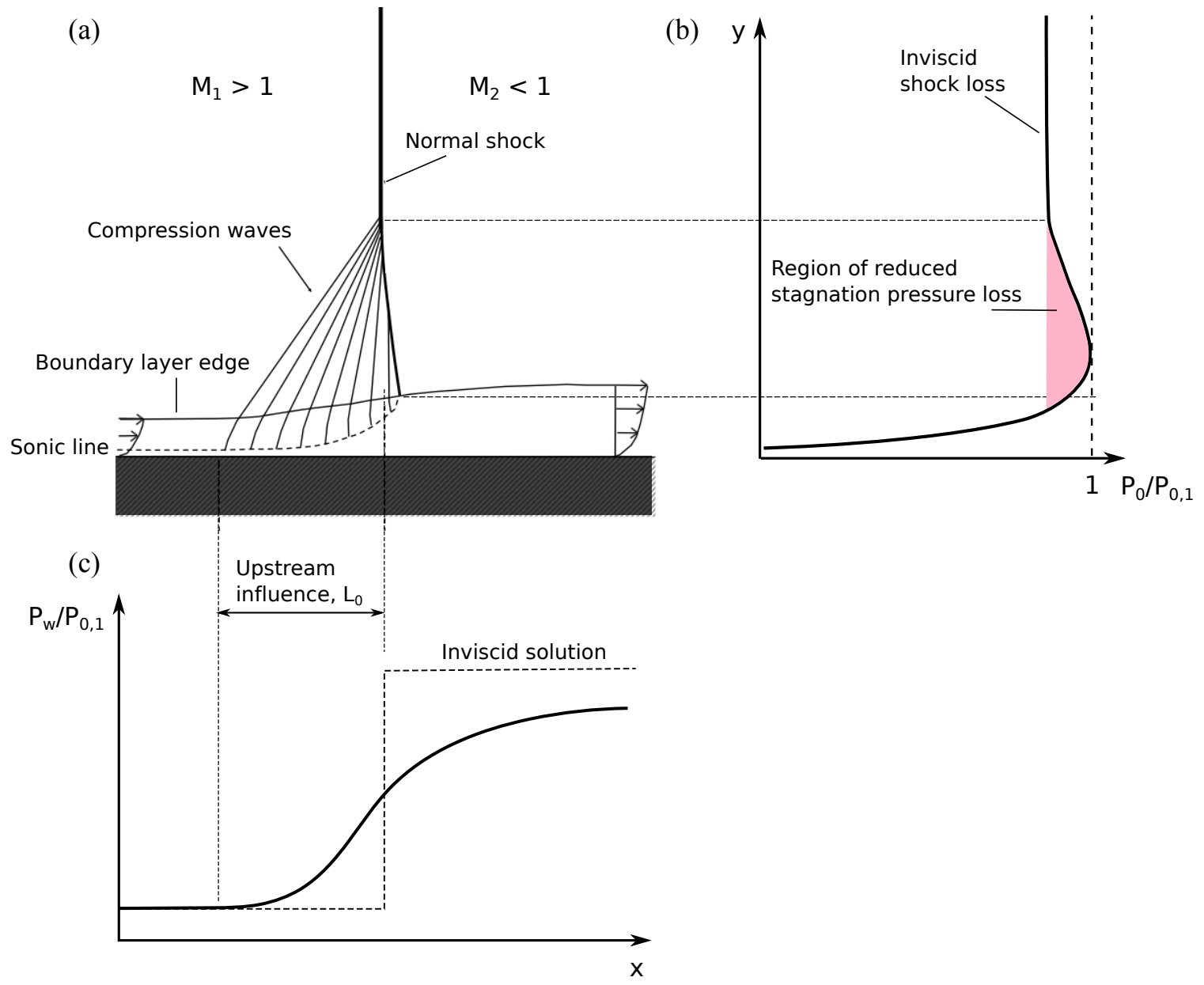

Fig. 2.2 The flow field of an attached normal SBLI: $(a)$ sketch of the interaction structure; (b) wall-normal stagnation pressure distribution; $(c)$ streamwise wall pressure distribution, adapted from [7]. 


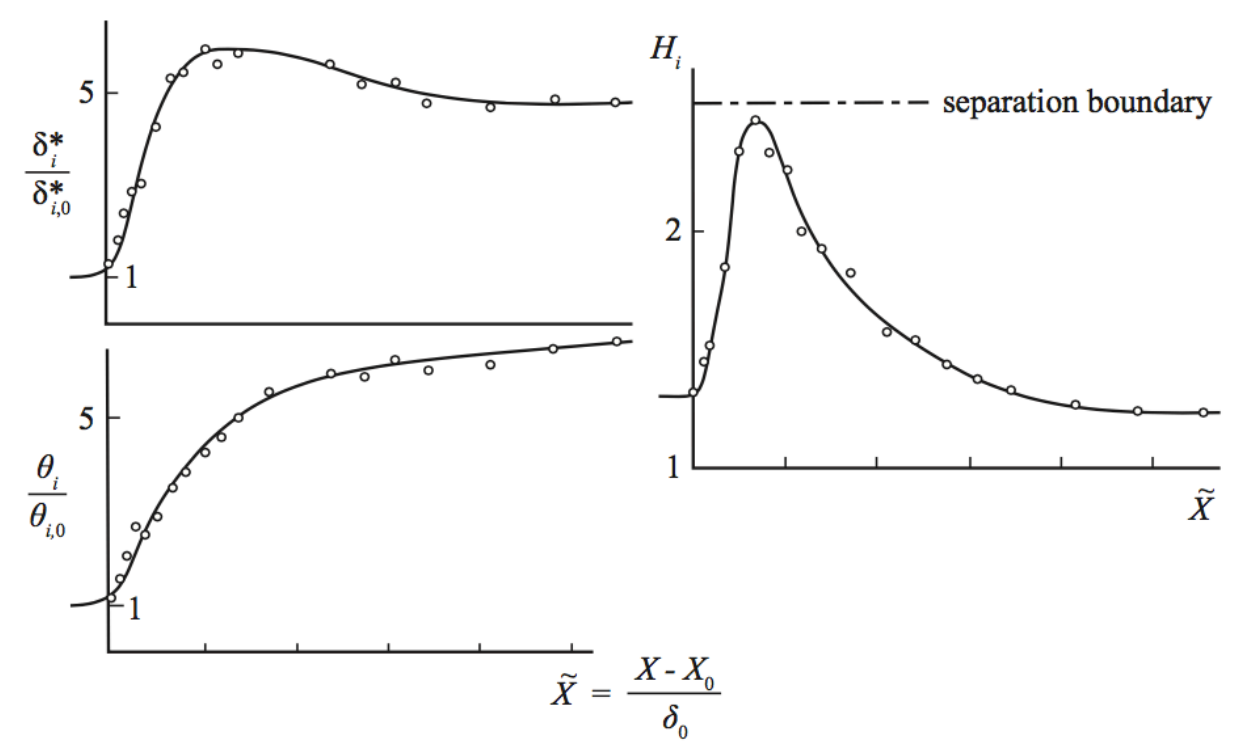

Fig. 2.3 Variation of boundary layer parameters through an attached normal SBLI, subscript 0 refers to values upstream of the interaction, from [42].

coalesce into a 'smeared' shock foot, and progressively weaken the local shock near the boundary layer, as more of the compression has occurred already. The height of this smearing interaction is typically of the order of a few boundary layer thicknesses $[1,6]$.

The wall-normal stagnation pressure profile downstream of the interaction is shown in figure $2.2 \mathrm{~b}$. The total pressure loss in the free-stream matches that of the inviscid interaction [116]; while in the boundary layer losses are caused by viscous losses [4]. A narrow region of reduced losses is observed above the boundary layer, where part of the normal shock is replaced by compression waves.

A typical streamwise wall pressure distribution across the interaction is given in figure 2.2c. A noticeable feature is that the shock-imposed abrupt pressure jump is spread over a streamwise distance, thus reducing the adverse pressure gradient and allowing the boundary layer to remain attached. This effect can be scaled by the upstream influence length $L_{0}$, defined by Délery [42] as the distance between the interaction onset (i.e. the location where the wall pressure starts to rise) and the shock foot in the inviscid-flow model. It is also seen that downstream of the shock the pressure rise is much gentler in comparison to the supersonic portion of the interaction, and that it takes a long time before the theoretical normal shock value is reached at the wall $[6,7]$.

Figure 2.3 shows the variation of the integral boundary layer parameters through an attached SBLI. The displacement thickness $\delta_{i}^{*}$ rises rapidly at the interaction onset, cor- 
responding to the displacement effect discussed above; whereas $\theta_{i}^{*}$ experiences a more moderate increase. This leads to a peak in $H_{i}$ around the shock impingement location. A rehabilitation process follows, during which $H_{i}$ drops and returns to an equilibrium value [41].

\section{Separated interaction}

As shock strength increases, a situation can be reached in which the turbulent boundary layer cannot withstand the pressure rise and separates. A more complex flow field forms, with the general structure shown in figure 2.4a (adapted from Seddon [112]).

(a)

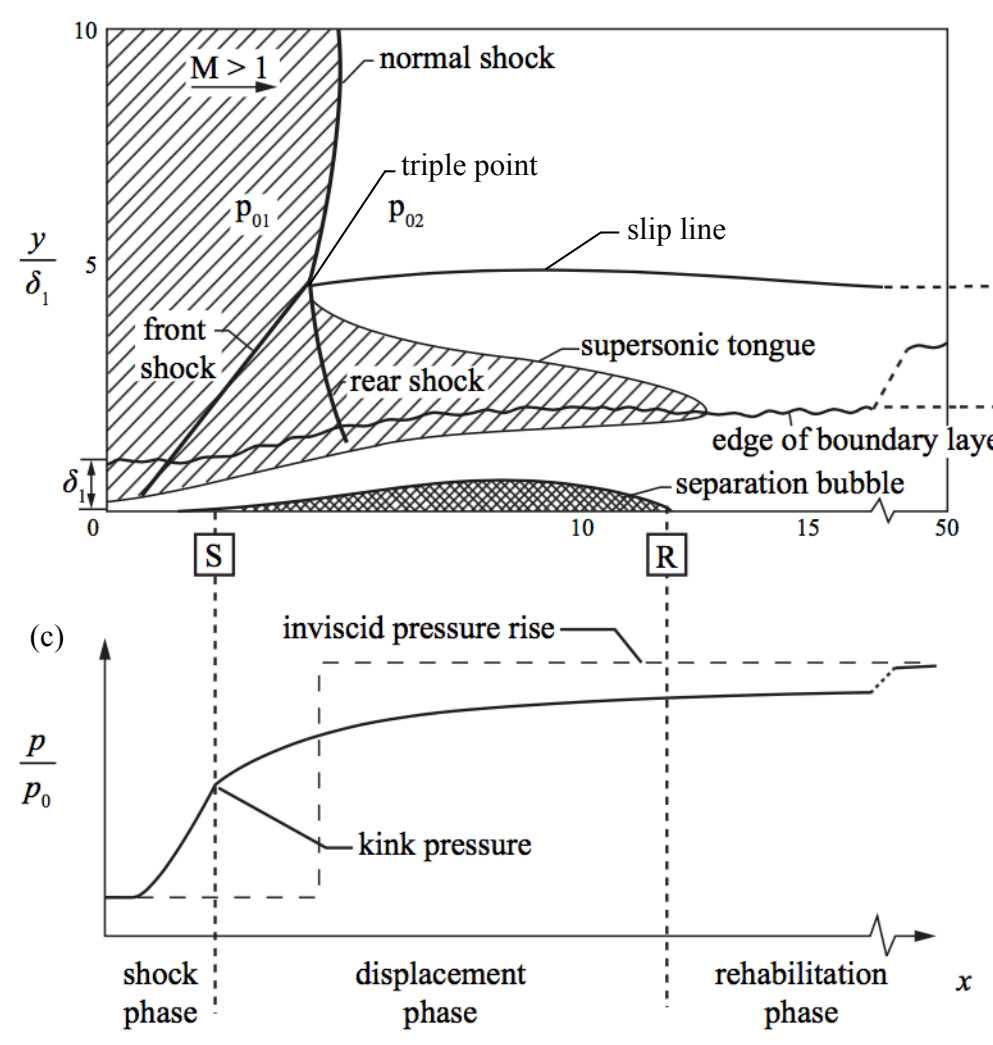

(b)

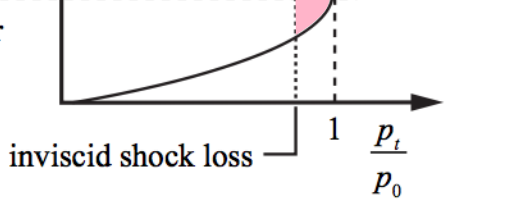

Fig. 2.4 The flow field of a separated normal SBLI: $(a)$ sketch of the interaction structure; (b) wall-normal stagnation pressure distribution; $(c)$ streamwise wall pressure distribution, adapted from Seddon [112].

At the separation point, a steep change in the flow direction is imposed on the outer flow which has to negotiate the separated bubble. This has the effect of compression waves 'bunching up' into an oblique shock, termed front or 'leading' shock. Initial compression through the front shock is however not sufficient to reach the pressure rise across the main shock, and a second shock is hence generated to form the rear 'leg' of the shock structure. The 

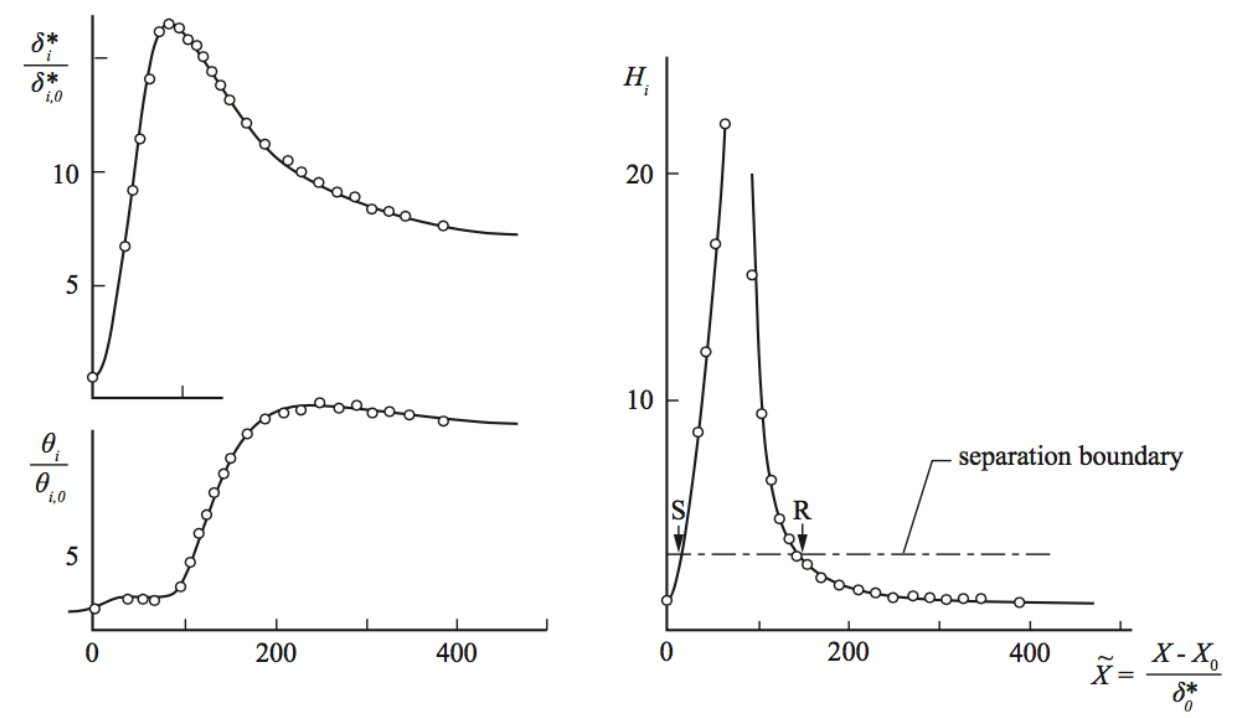

Fig. 2.5 Variation of boundary layer parameters through a separated normal SBLI, from [42].

front and rear legs meet the normal shock wave at the 'triple point'. This flow organization is referred to as a 'lambda-shock' structure for its similarity to the Greek letter $\lambda$. Another element commonly observed in a separated interaction is a secondary region of supersonic flow behind the $\lambda$-foot, known as the 'supersonic tongue' $[7,74,111,112]$.

As shown in figure $2.4 \mathrm{~b}$, the flow passing through the bifurcated $\lambda$-foot experiences a reduced total pressure loss compared to the flow through the main shock, indicating conditions with different entropy rises above and below the triple point. A sheet of vorticity (i.e. the slip line) is hence created to separate the two flow states (same pressure and flow direction however different velocity), which persists far downstream and smooths out the stagnation pressure discrepancy via flow mixing [74].

The surface pressure distribution (figure 2.4c) can be broken down into three distinct streamwise phases according to Seddon [112]. The 'shock phase' extends from the initial compression to the separation point. There is a significant pressure jump across the leading shock leg, featuring a 'kink' at separation in the profile (generally occurring at $\left.p=1.4-1.45 p_{1}[6,101,112]\right)$. The 'displacement phase' continues with a slow pressure rise, corresponding to the development of a bubble. The 'rehabilitation phase' is everything downstream of the interaction domain, where the pressure jump predicted by inviscid relations is reached only after a long streamwise distance. 
The boundary layer behaviour across a separated SBLI is depicted in figure 2.5. It is observed that changes in the integral parameters are similar to the attached case (figure 2.3), however in a more severe manner. The value of $H_{i}$ is seen to increase rapidly and pass $H_{i} \approx 2.5$, an indicator of incipient separation proposed by Délery [42] and White [137]. It then disappears off the scale, denoting the extremely distorted velocity profiles during separation, before falling again when reattachment begins.

\subsubsection{Oblique SBLIs}

\section{Attached interaction}

In attached incident-reflecting shock interactions, viscous effects are a mere correction to the inviscid solution. The general flow structure hence closely resembles the inviscid model, as shown by the schlieren photograph in figure 2.6.

The typical interaction structure, following the work of Délery and Bur [40], is illustrated in figure 2.7a. The pressure rise induced by the incident shock is experienced upstream through the same transmission mechanism as in the normal SBLIs. The dilatation of the boundary-layer subsonic region acts like a 'viscous bump', inducing compression waves that quickly coalesce to form the reflected shock. The incident shock penetrates into the rotational supersonic boundary layer and refracts into a series of compression waves (refer to Henderson [70] for the detailed mechanism). On approaching the wall, the upstream $M$ decreases. The incident-wave system hence bends and becomes steeper until vanishing at sonic line, where compression waves are reflected as expansions. The reflected shock combines with these expansion waves to deflect the velocity vector back to the inflow direction.

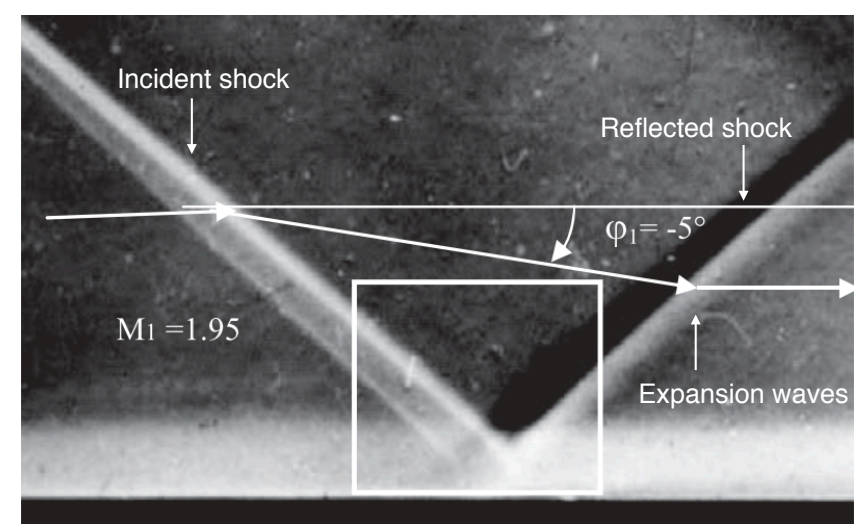

Fig. 2.6 Schlieren photograph of an attached oblique shock reflection, adapted from Dèlery [7]. 
(a)

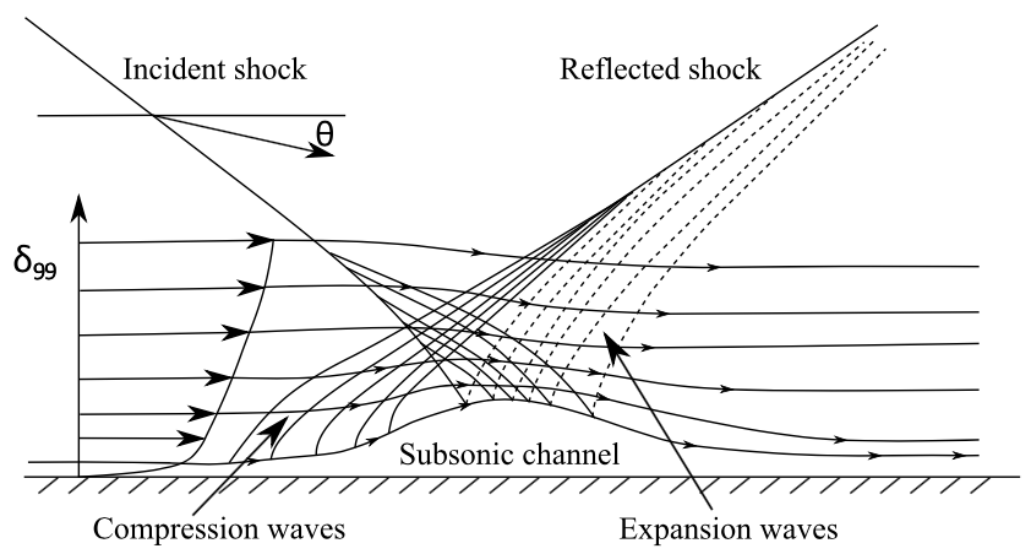

(b)

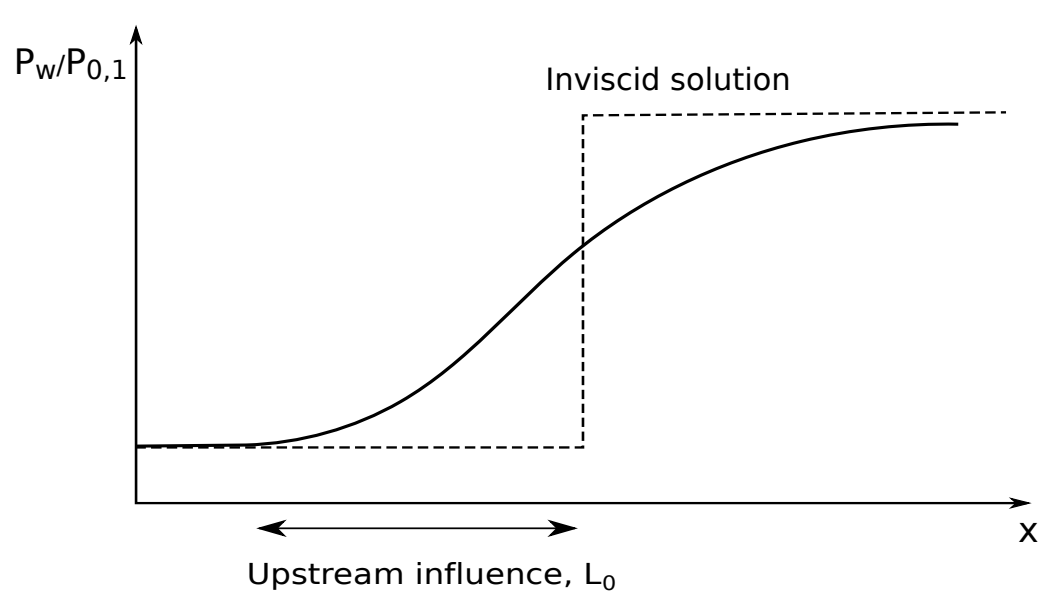

Fig. 2.7 The flow field of an attached oblique SBLI: $(a)$ sketch of the interaction structure, reproduced by Giepman [60] following Délery and Bur [40]; (b) streamwise wall pressure distribution.

The smeared wall pressure rise shown in figure $2.7 \mathrm{~b}$ is similar to that of an unseparated normal interaction (figure 2.2c), exhibiting an upstream influence length on the order of the boundary-layer thickness. Downstream of the inviscid pressure jump, pressure increases steadily and tends towards the inviscid value.

\section{Separated interaction}

The flow field adopted by the separated oblique SBLI is markedly different from the purely inviscid case, as visualised in figure 2.8. Compared to the attached scenario (figure 2.6), the presence of a separated region leads to an noticeable upstream shift of the separation shock, which now intersects the incident shock outside of the boundary layer. The incident shock is seen to penetrate the separated viscous flow, where it is once again reflected as an expansion 
fan. In addition, a considerably thicker boundary layer is created after interacting with the shock system, indicating the detrimental effects on the flow as a result of the separation of the turbulent boundary layer.

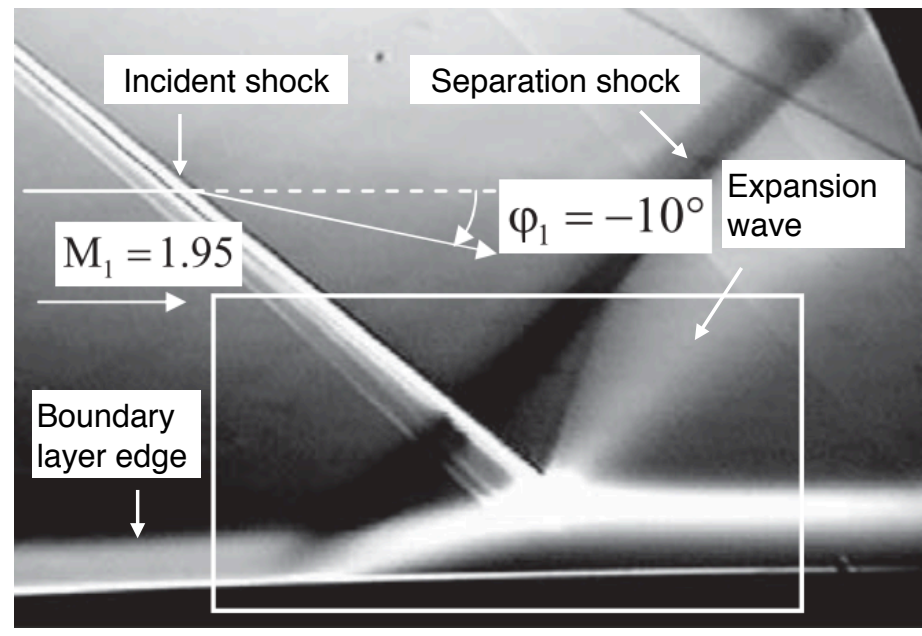

Fig. 2.8 Schlieren photograph of a separated oblique shock reflection, adapted from Dèlery [7].

A schematic illustration of the interaction structure is provided in figure 2.9a. It can be seen that downstream of $\mathrm{H}$, the intersection point of the incident and separation shocks, flows traversing different sets of shocks are separated by a slip line. A shear layer develops along this slip line, ensuring a continuous variation of the flow properties above and below H. The incident shock combined with the expansion waves provoke a deflection of the separated shear layer (emanating from the separation point $S$ ) towards the wall. This, together with the strong mixing occurring at the separation bubble edge, helps to reattach the flow at $\mathrm{R}$. There, a new deflection takes place with the formation of compression waves, which eventually coalesce into a reattachment shock. Délery et al. [41] has stated that the compression at reattachment is in general more smeared than at separation, and hence the reattachment shock forms further from the wall and can be quite smeared-out even in the outer inviscid flow. This is in agreement with the schlieren image shown in figure 2.8, where the reattachment shock cannot be clearly identified.

A major consequence of the separated interaction is to divide the shock-induced pressure jump into two 'steps', as shown in figure $2.9 \mathrm{~b}$. The wall pressure first increases rapidly at separation, followed by a plateau associated with the separation bubble. A second, more gentle compression then occurs around reattachment. A numerical study by Matheis and Hickel [85] showed that although this pressure plateau is quite well-defined for strong interactions with large separation bubbles (see $S B L I_{3-5}$ shown in figure 2.10), it can be subtle for cases with a reduced separation length $\left(S B L I_{1-2}\right)$. Such a finding holds true 
(a)

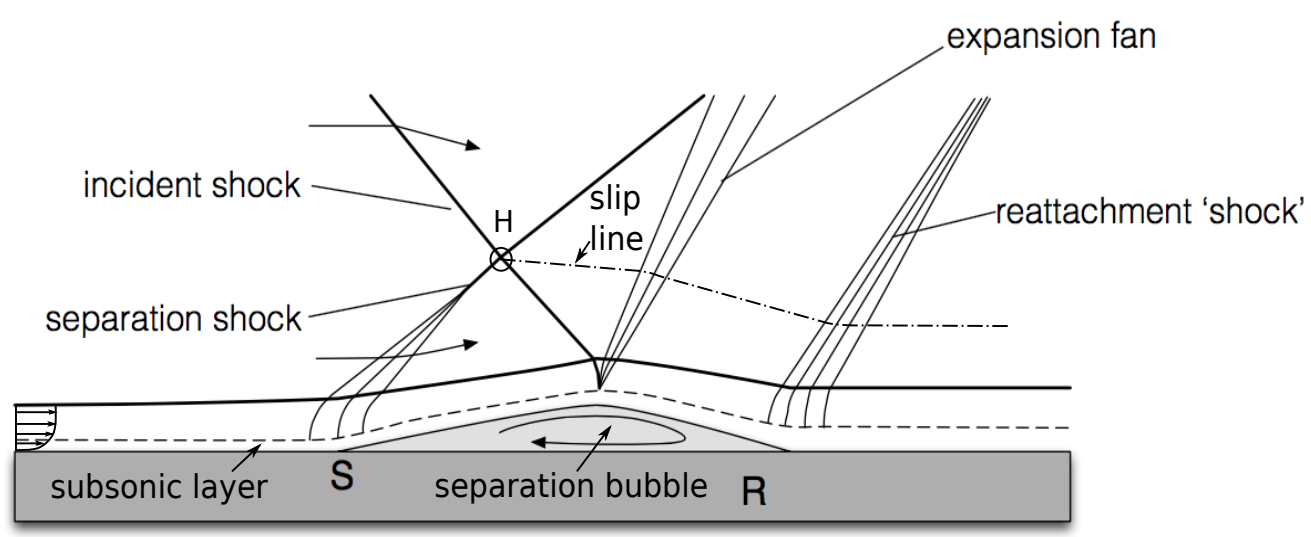

(b)

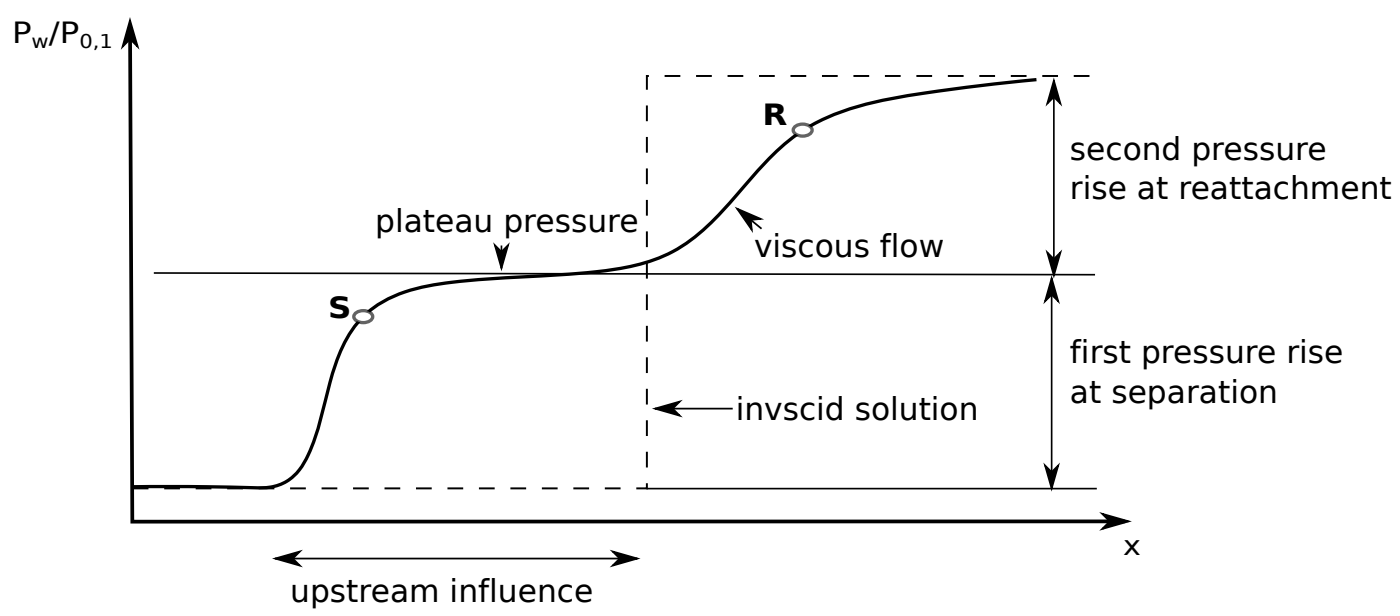

Fig. 2.9 The flow field of a separated oblique SBLI: (a) sketch of the interaction structure; (b) streamwise wall pressure distribution, based on the work of Délery and Bur [40].

for experimental measurements in the regime of small-scale separations (refer to Délery [42], Deleuze [46], Hayashi et al. [68], Laurent [78], Law [79]), where a clear plateau region is rarely observed and instead a more gradual pressure rise is seen. In such a scenario, the first inflection point in the static pressure distribution indicates for the location of the separation point [79].

By comparing the separated SBLI induced by a normal shock (figure 2.4) with an oblique shock reflection (figure 2.9), it is seen that the flow behaviour up to the separation point, in particular the wall-pressure distribution, is quite similar. Such a similarity has also been observed in many other types of SBLIs, where the major part of the interacting flow upstream of the separation bears a certain resemblance for rather distinct configurations [16, 31, 41, 55, 115]. This observation led Chapman et al. [31] to formulate the free interaction theory, as described by Délery et al. [41]:'Everything happens as if the flow were entirely 


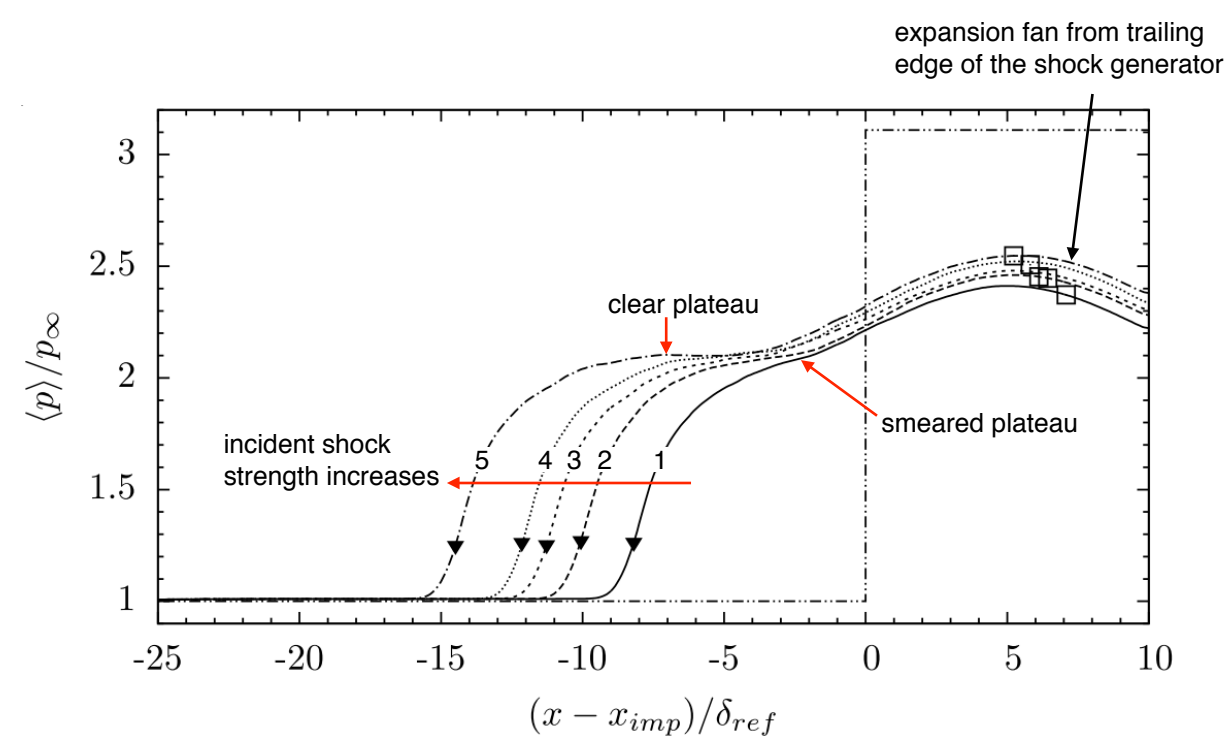

Fig. 2.10 Computations of wall pressure distribution for oblique shock reflection in $M_{0}=2$ flow. The incident shock strength increases from condition 1 to 5, with symbols denoted $(\boldsymbol{\nabla})$ separation point and $(\square)$ impingement location of the first expansion wave from the downstream wedge corner, adapted from Matheis and Hickel [85].

determined by its properties at the onset of interaction.' In oblique SBLIs, this theory has been experimentally well confirmed by Green [63] and Délery et al. [41], showing that the strength of the separation shock is independent of the incident shock that causes separation. Matheis and Hickel [85] found that flow deflection angle across the separation shock also remained constant for different incoming shock strengths. While the initial part of the SBLI is a self-induced free-interaction process between the boundary layer and the outer inviscid stream, the reattachment stage depends on the overall pressure rise. An increase in the incident-shock strength therefore requires a greater pressure jump at reattachment. The extent of the separation region hence increases, to allow the viscous shear layer to build up more momentum before reattachment. Consequently, the shock-induced separation length grows proportionally to the pressure rise at reattachment [7].

\subsection{Parameters governing the shock-induced separation}

Shock-induced separation is highly undesirable in engineering applications. For transonic airfoils, the supersonic region is terminated by a normal shock. Flow breakdown on wings is essentially driven by separations, which can lead to significant shock oscillations and induce severe loading variations on the aircraft structure [103]. In supersonic mixed-compression inlets, deceleration is achieved by a number of oblique shock waves and a terminal normal 
shock. The high adverse-pressure-gradient flow field created by such a shock system may trigger boundary-layer separation, resulting in increased total pressure loss and flow unsteadiness. In extreme circumstances where the separated region in core flow grows too large the inlet will un-start, causing a considerable reduced thrust or even engine shut down [71].

Driven by these detrimental effects, predicting the incipient separation has been a task investigated by numerous studies. Parameters contributing to its occurrence are examined to gain an insight into underlying physics.

\subsubsection{Prediction of incipient separation}

Délery [42] collected experimental data of the normal shock-induced separation limit on a flat plate, and plotted them as $\mathrm{M}$ against $H_{i 0}$, the incompressible shape factor at the interaction onset (figure 2.11). It can be seen that in nominally two-dimensional transonic flows, a turbulent boundary layer generally separates at shock strengths above Mach 1.3 with almost no dependence on $H_{i 0}$. The observed insensitivity to the shape factor can be explained when taking the upstream influence mechanism into consideration. With increasing $H_{i}$, lower momentum of the near-wall fluid leads to a reduced resilience to separation; however $y_{s}$ also increases to exhibit a larger $L_{0}$, which causes a greater smearing of the pressure rise [42]. This in turn delays separation (as discussed before in section 2.1.3). Often, these two contradictory effects cancel each other.

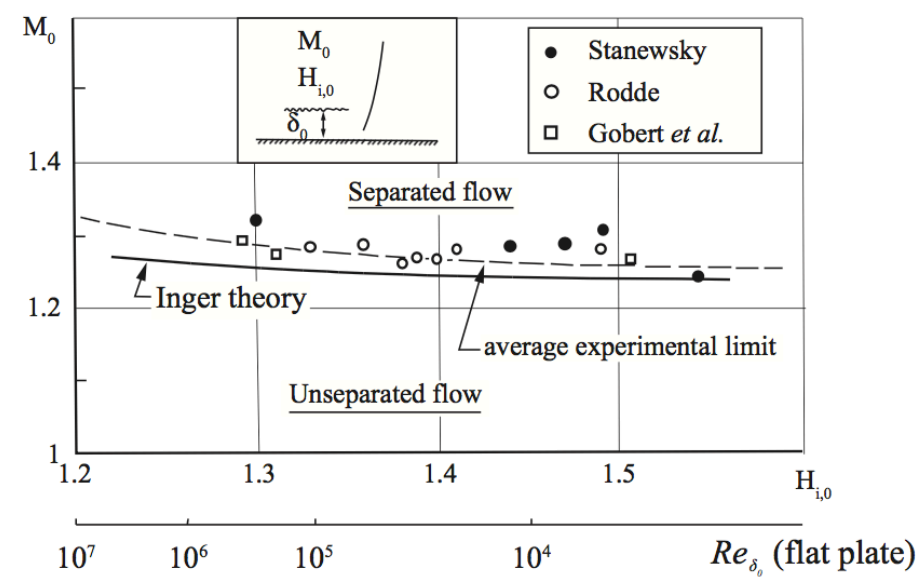

Fig. 2.11 The onset of normal shock-induced separation, from [42].

Bruce et al. [25], based on the work of Sajben et al. [109], conducted a more complete survey of published experimental results from different facilities, as shown in figure 2.12a. Here significant variation exists for the critical Mach numbers inducing separation, with a 


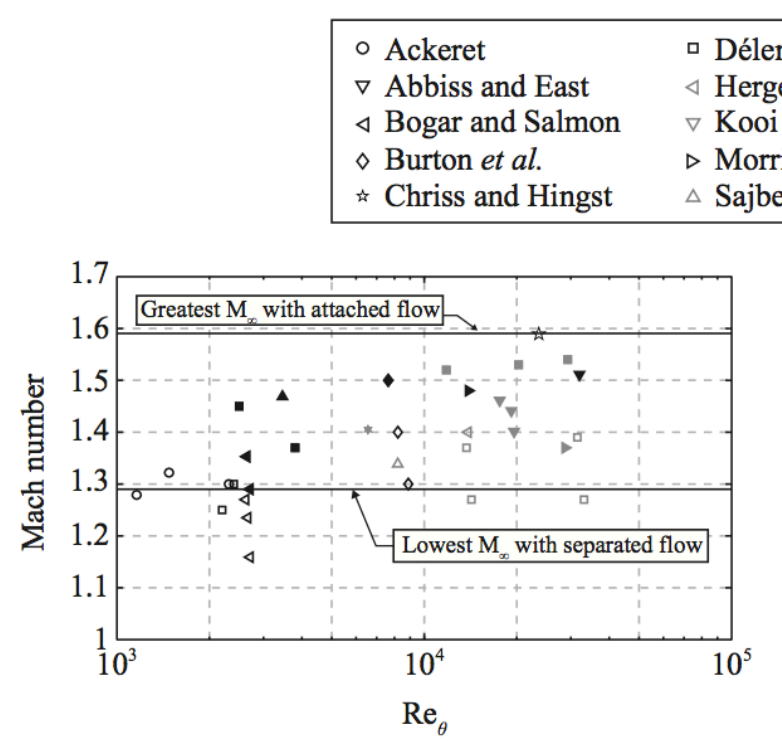

(a)

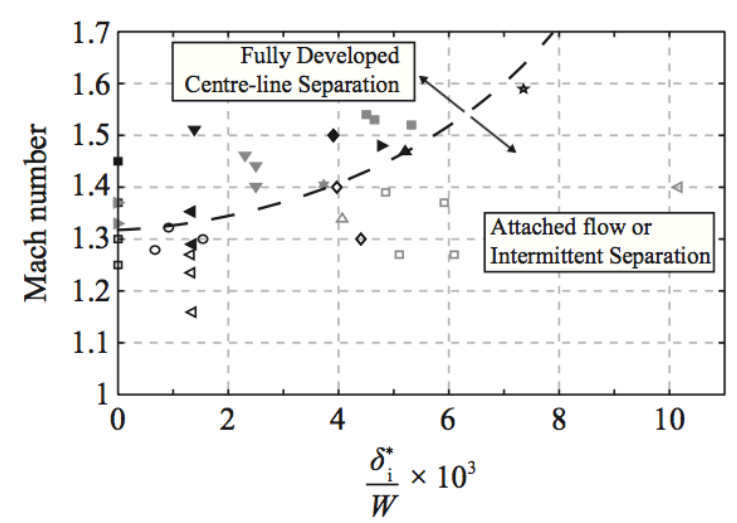

(b)

Fig. 2.12 The onset of shock-induced separation in transonic channel flows, from Bruce et al. [25]: (a) correlation with $R_{e \theta}$, following Sajben et al. [109]; (b) improved parameter $\delta_{i}^{*} / W$ accounting for confined effects. Open symbols represent attached central flow, filled symbols for separated central flow.

documented normal SBLI remaining attached as high as $M=1.59$ (study of Chriss et al. [32]). While the wide scatter cannot be explained by a Reynolds number dependency, the authors suggested that this was due to separation in the corner of a rectangular wind tunnel. The ratio of the incoming boundary layer displacement thickness to the tunnel width $\left(\delta_{i}^{*} / w\right.$, termed viscous aspect ratio) was found to capture the significance of corner separations, enabling the data to be reconciled into a separation criterion incorporating three-dimensional effects (figure 2.12b). Higher values of $\delta_{i}^{*} / w$ correspond to more confined facilities with relatively larger corner flow separations, which appears to suppress the central separation and push the flow towards the attached domain.

Burton and Babinsky [28] proposed that corner flows affected the onset of central separation through modifying the APG experienced by the floor boundary layer (the physical mechanism will be explored in section 2.3). This is in agreement with the study of Délery [42], who points out that the adverse pressure gradient is an influential factor governing the occurrence of separation. 


\subsubsection{Importance of adverse pressure gradient}

An experimental investigation of supersonic SBLIs conducted by Smith and Smits [118] provides evidence that shock-induced separations are sensitive to manipulations of the APG. Two compression ramps, model A with a sharp corner and model B with a rounded corner, were designed such that the flow experienced the same changes in direction and pressure, but different pressure gradients. In both experiments, the ramps did not span the wind tunnel, isolating the ramp distortions from the pronounced sidewall effects, hence maintaining the relative two-dimensionality of the boundary-layer flow through the SBLI.
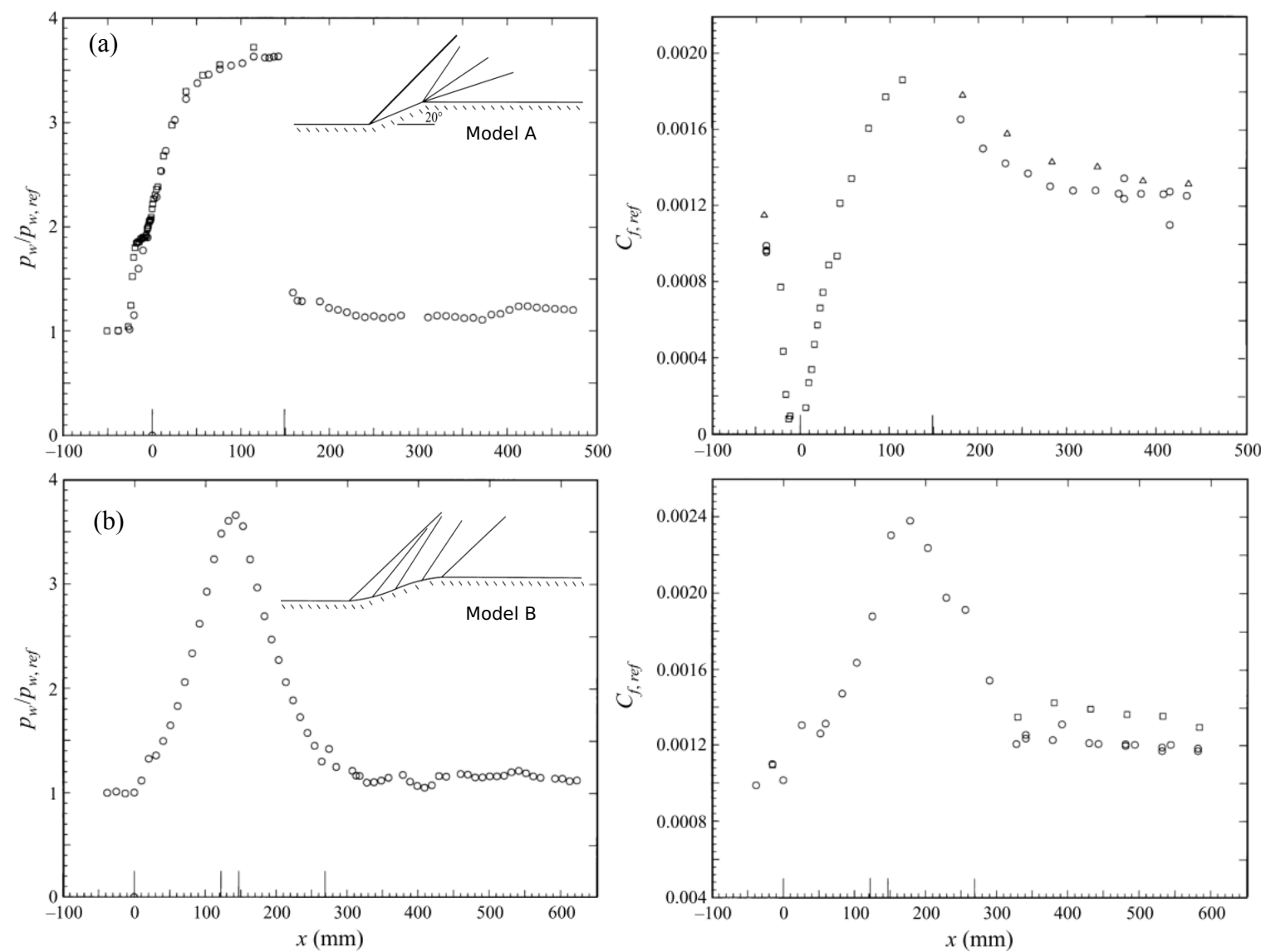

Fig. 2.13 Wall pressure $\left(p_{w}\right)$ and wall friction $\left(c_{f}\right)$ distribution measured by Smith and Smits [118] for two compression ramp arrangements: $(a)$ model A as a sharp corner; $(b)$ model $\mathrm{B}$ as a rounded corner. The beginning and end of the concave and convex curvatures are marked on the abscissa with vertical lines.

As shown by the pressure measurements in figure 2.13, model A and model B present a similar pressure jump of $p_{w} / p_{w, r e f}=3.7$ across the interactions, reaching the inviscid level for $20^{\circ}$ of turning at $M=2.9$ ( $p_{w}$ and $p_{w, r e f}$ refer to the local and incoming flow wall pressures respectively). However, the pressure increases over different streamwise distances, 
with $135 \mathrm{~mm}$ for model A compared to $150 \mathrm{~mm}$ for model $\mathrm{B}$. The longer interaction length for the rounded corner consequently imposes a less severe adverse pressure gradient on the boundary layer. The corresponding wall skin friction distributions are also provided $\left(C_{f, r e f}\right.$ is the local wall friction normalised by $\frac{1}{2} \rho_{r e f} U_{r e f}^{2}$ ). It can be seen that only for model A does $C_{f, r e f}$ decrease rapidly approaching the compression ramp and drop to 0 , indicting that the flow is separated in the corner. The presence of the separation bubble has been confirmed by Settles et al. [114], using the identical ramp in the same facility. Their results show that for a fixed pressure rise, the magnitude of the APG can determine whether the flow separates or remains attached.

Shang et al. [115] noted that there were great similarities between the compression ramp and incident oblique-shock interactions. The pressure distribution produced by an incident shock was seen to be almost identical to that of a compression corner with a double angle. Thus, the separation criterion for compression-ramp flows can also be applied to oblique shock reflections.

The above discussion shows that while the shock strength at the onset of a SBLI is critical in determining when flow separation occurs, the incompressible shape factor $\left(H_{i 0}\right)$ and Reynolds number $\left(R_{e \theta}\right)$ ahead of the interaction only present weak influence. A more significant factor appears to be the adverse pressure gradient imposed on the flow. For a similar pressure jump, a reduced APG can result in a turbulent boundary layer remaining attached across the interaction when it might otherwise be separated. Geometrical differences among wind-tunnel facilities are also found to affect the onset of separation. In normal SBLIs this is believed to be due to the compression waves caused by corner separations, as discussed in the next section. 


\subsection{Corner effects in rectangular channels}

\subsubsection{Supersonic corner flows}

Mixed-compression inlets, desirable for aircraft faster than $M=2$, often take the form of rectangular ducts. This geometric feature is shared by the working section of high-speed wind tunnels, the main research tool for SBLI investigations.

Supersonic turbulent flows in a straight channel are characterized by strong secondary flows in the four streamwise corners, which are initiated by Reynolds stress gradients acting in the near-wall region $[19,106]$. As shown in figure 2.14, the secondary flows usually comprise of two counter-rotating vortices, directing the velocity vectors toward the wall along a corner bisector [38]. The maximum transverse velocity in the cross-plane was measured to be 3\% of the free-stream by Mojola and Young [92]. These vortices have been observed to distort the primary supersonic flow, and deviate it from the canonical twodimensional structure $[39,84]$. Gessner [59] proposed that secondary flows acted to convect mass, momentum, vorticity and energy between the core and the corner regions. Morajkar et al. [94] suggested that corner separations can also affect the performance of internal flows through reducing the effective channel area.

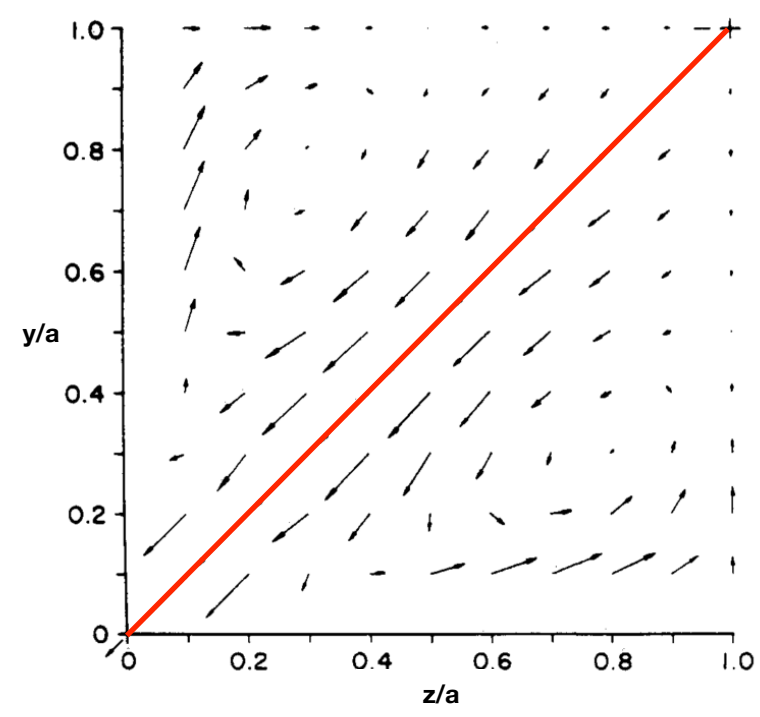

Fig. 2.14 Corner vortices of supersonic turbulent flow in a square duct, adapted from Davis and Gessner [38]. $a$ represents duct half width.

When a shock wave is placed in a rectangular channel, the flow is subjected to a strong streamwise adverse pressure gradient. In such a scenario, corner flows are generally more likely to separate or separate further upstream (referred to as corner separation) compared to the central regions of the channel walls (centreline separation). This is because corner 
boundary-layers typically feature considerable regions of low-momentum as a result of the viscous interactions with two surfaces. A highly three-dimensional SBLI is hence created in the corner [3, 107, 110].

The approach taken by many investigations in wind tunnel tests is to focus their measurements around the mid-span, assuming that the flow away from the sidewall can be treated as nominally two-dimensional. However more recently a possible link between corner separations and centreline flow has been suggested by studies for a wide range of Mach numbers, Reynolds numbers and configurations, including the findings of Kussoy et al. [76]; Gaitonde and Shang [56]; Dupont et al. [49]; Doerffer et al. [47]; Helmer et al. [69].

The following sections will discuss the influence of corner separations on central flows for normal and oblique interactions respectively. Critical point theory (Legendre [80]) is also briefly revisited to understand three-dimensional separation topologies.

\subsubsection{Corner effects for normal SBLIs}

The three experiments shown in figure 2.15 were conducted in different wind-tunnel facilities, all investigating the onset of separation for a normal SBLI on a flat plate. Despite these tests being at roughly similar Mach numbers and Reynolds numbers, the oil-flow visualisation exhibited clearly different flow patterns.

The flow field seen in figure 2.15(a) was produced by Burton et al. [29] at Cambridge University, with a unit Reynolds number of $2.41 \times 10^{7} \mathrm{~m}^{-1}$. Here the normal shock induced a small amount of separation around the tunnel floor centreline in a $M_{\infty}=1.5$ flow, while a larger separated region was observed in each of the corners. The centreline and corner separations are bounded by a dot-dashed line and a dashed line respectively. The vertical line indicates the shock location.

Conversely, for a lower incoming Mach number (1.45) and a similar Reynolds number $\left(1.57 \times 10^{7} \mathrm{~m}^{-1}\right)$, Doerffer and Szwaba [48] reported a heavily separated central flow, as shown in figure $2.15 \mathrm{~b}$. The corner separation was observed to be relatively small.

In the NASA Lewis Research Centre supersonic tunnel, boundary layer along the floor centreline was seen to remain attached through a $M_{\infty}=1.59 \mathrm{SBLI}$, with a Reynolds number of $1.50 \times 10^{7} \mathrm{~m}^{-1}$ (figure $2.15 \mathrm{c}$ ). In this experiment extensive flow separations were presented in corners.

These results highlight an interesting pattern: in a rectangular channel, early centreline separation is associated with a small corner separation (figure $2.15 \mathrm{~b}$ ), whereas the facility with attached central flow has sizeable regions of separated corner flows (figure 2.15c).

For SBLIs focused on flat plates, researchers have generally taken the flow behaviour around the centre-span as areas of interest. Such flow visualisation on corner flow is hence 
(a)

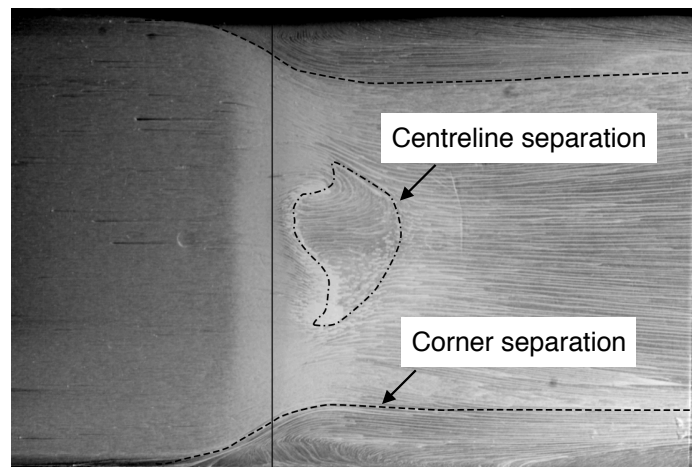

(b)

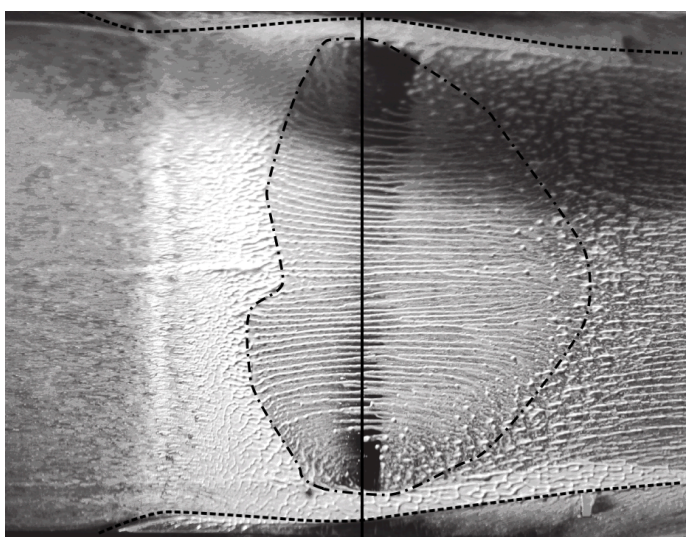

(c)

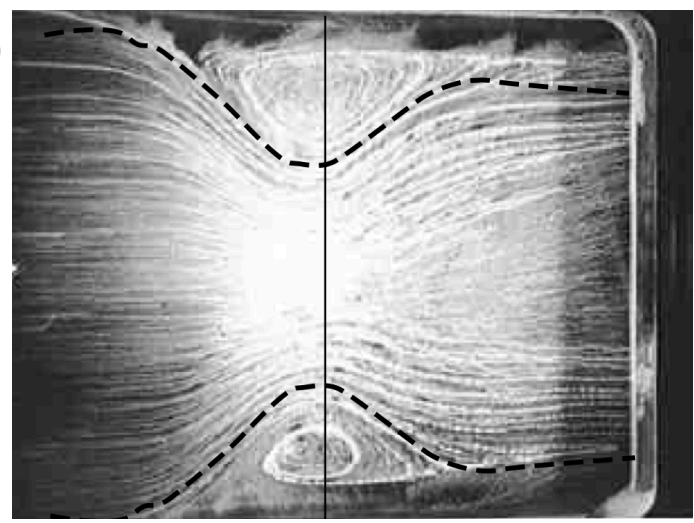

Fig. 2.15 Oil-flow visualisation for normal SBLIs in supersonic wind tunnel facilities with rectangular cross-sections: (a) $M_{\infty}=1.5$ by Burton et al. [29]; (b) $M_{\infty}=1.45$ by Doerffer and Szwaba [48]; (b) $M_{\infty}=1.59$ by Chriss et al. [32]. Flow from left to right.

not readily available for the majority of published experiments. This led to the parametric study of Bruce et al. [25], who proposed that the extent of corner separation can be scaled at first order with the boundary layer displacement thickness along the tunnel floor centreline $\left(\delta_{i}^{*}\right)$. The viscous aspect ratio $\left(\delta_{i}^{*} / w\right)$, as previously discussed in section 2.2.1, can therefore be used to estimate the three-dimensionality of a particular flow due to corner effects. The 
(a)

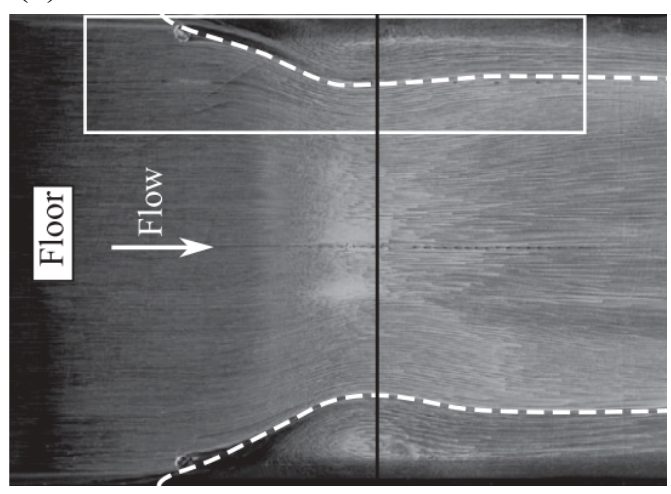

(b)

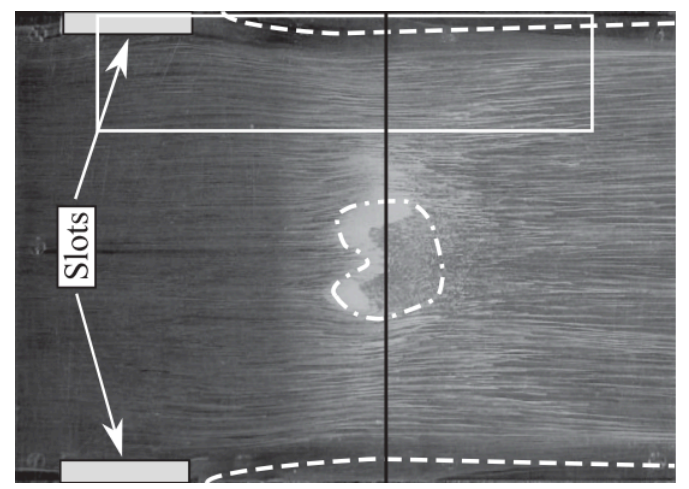

Fig. 2.16 Oil-flow patterns on the tunnel floor for a $M_{\infty}=1.4$ shock, measured by Bruce et al. [25]: (a) baseline flow; (b) corner-suction applied.

(a)

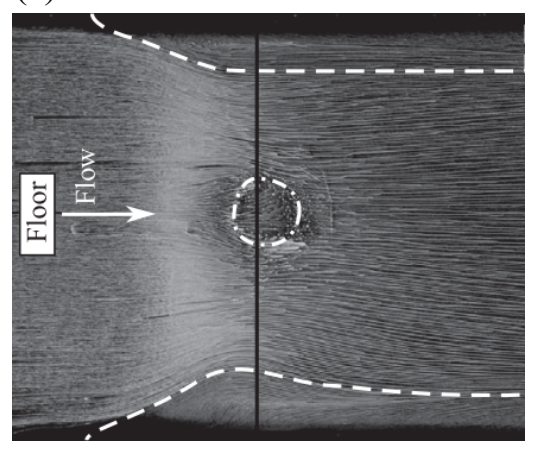

(b)

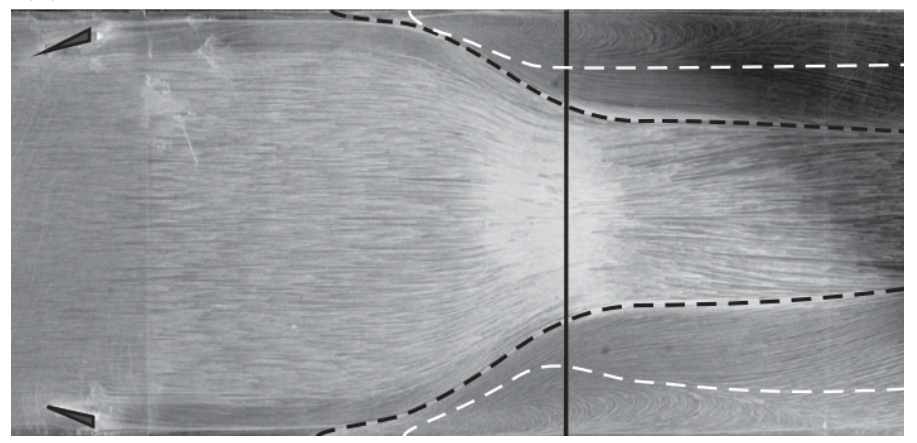

Fig. 2.17 Oil-flow patterns on the tunnel floor for a $M_{\infty}=1.5$ shock, measured by Bruce et al. [25]: (a) baseline flow; $(b)$ corner-VGs applied.

authors went further to manipulate the relative size of corner separations for an established normal SBLI. As seen in figure 2.16, when suction was applied to reduce the magnitude of corner separations, a separated bubble was introduced in the previously attached central region. On the contrary, figure 2.17 shows that an increased corner effect can eliminate the centreline separation. These results support the link presented in figure 2.12b (plotted by data from different facilities), with larger separated corner regions delaying the quasi-twodimensional separation onset along the centreline.

Burton and Babinsky [28] found that a conical compression fan was generated due to the displacement effect of the corner separation. A three-dimensional SBLI structure accounting for corner effects has been proposed and sketched in figure 2.18. These compression waves interact with the normal shock across the working section to change the size of the $\lambda$-foot. The variation in the $\lambda$-foot size then alters the APG experienced by the floor boundary layer. For flows where the corner separations are extensive (e.g. figure 2.15c, figure $2.17 \mathrm{~b}$ ), the 


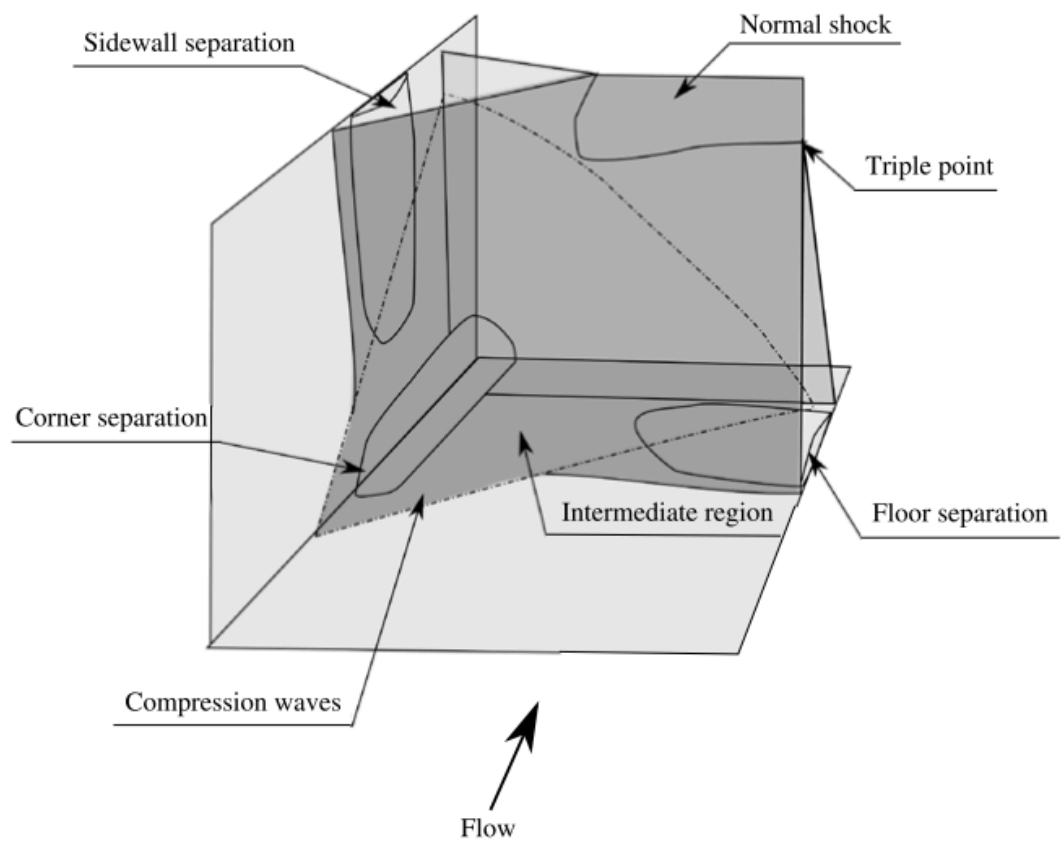

Fig. 2.18 Three-dimensional diagram of normal SBLI in a corner region, proposed by Burton and Babinsky [28].

compression fan can reach the centreline. In such a case, the distinct $\lambda$-foot shock structure is smeared into a series of compression waves, which in turn can reduce the APG to such an extent that the local flow remains attached.

This modification of the $\lambda$-shock region is in agreement with the findings of previous authors such as Weber et al. [136] and Handa et al. [65]. Supported by velocity measurements, they stated that the three-dimensional shock structure produced in the supersonic region was a more important mechanism compared to the increased blockage effect from corner separations in the subsonic area, the latter of which had been adopted as a possible explanation of corner effects by Garnier [58]. 


\subsubsection{Corner effects for oblique SBLIs}

Corner effects on incident-reflecting shock interaction inside a low-aspect-ratio duct were investigated by Eagle and Driscoll [51]. They concluded that their results were consistent with the general ideas of Burton and Babinsky [28], explaining that the compression waves generated at corners and sidewalls are the primary mechanism modifying the APG in space. Using stereo-PIV in various planes of all three orthogonal orientations, lateral velocities and vorticity fields were measured. This data set helps to determine the three-dimensional flow field with five separate vortices identified (as sketched in figure 2.19), showing how the flow structure adjusts to accommodate the redistribution of pressure gradients. Their work suggests that there may be significant similarities between normal and oblique SBLIs with respect to corner effects.

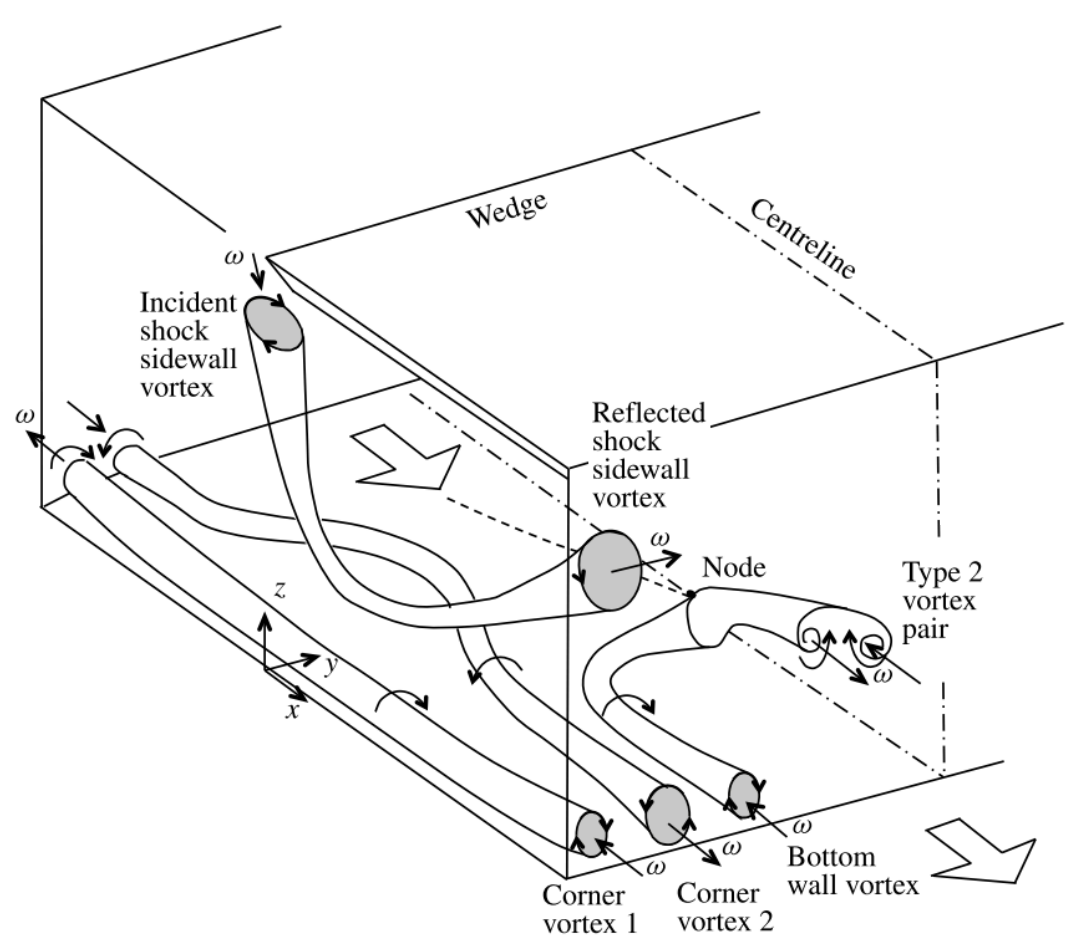

Fig. 2.19 Schematic three-dimensional flow field for an oblique SBLI in a rectangular duct, from Eagle and Driscoll [51].

Babinsky et al. [10] proposed that, as the corner compression waves (reduced to a single 'corner shock' for simplicity in figure 2.20a) passed through an oblique shock reflection, modification of the streamwise APGs might be determined by the relative position of the corner shock and the main incident shock wave. On the assumption that the viscous aspect ratio can capture this effect they hypothesised the dependency of centreline separation length $L_{\text {sep }}$ on $\delta / w$ as shown in figure $2.20 \mathrm{~b}$. This hypothesised increase and decrease in 


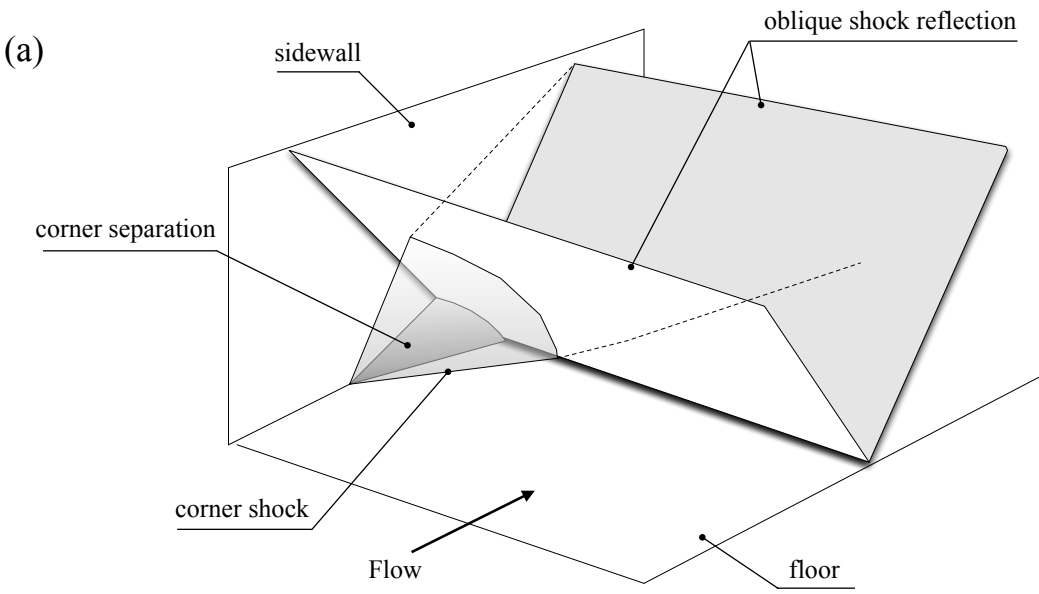

(b)

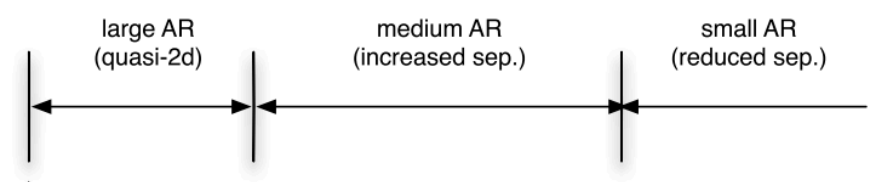

$\mathrm{L}_{\text {sep }}$ (centre)

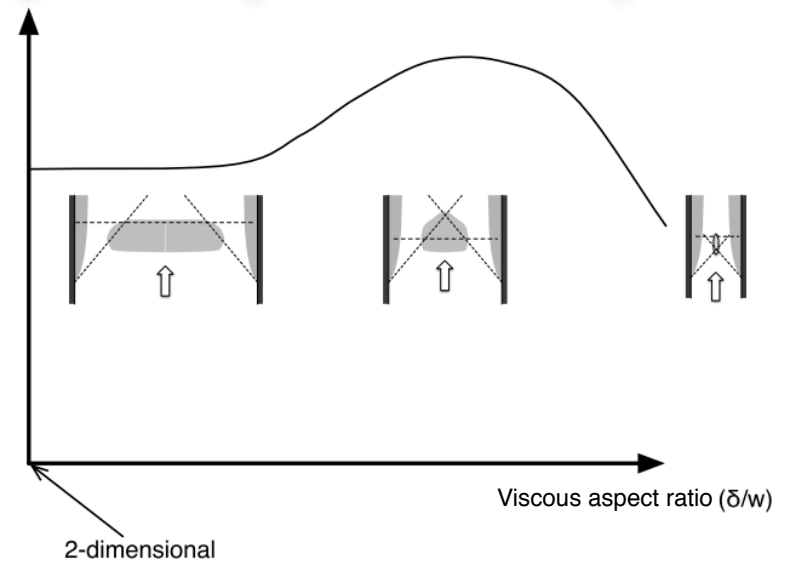

Fig. 2.20 Corner effects for a separated oblique shock reflection, adapted from Babinsky et al. [10]: (a) schematic diagram of the corner shock wave; $(b)$ proposed variation of separation lengths along the tunnel floor centreline as a function of viscous aspect ratio.

separation length with increasing viscous aspect ratio was computationally verified by the RANS simulations of Benek et al. [11], using an anisotropic $k-\omega$ model. Benek et al. [11] however have observed no gradual rise from the two-dimensional separation length even for flows of small viscous aspect ratios $(\delta / w \leq 0.05)$. On the contrary, quasi-two-dimensional behaviour was presented in the large-eddy simulations of Wang et al. [134] for relatively large wind tunnel widths. This discrepancy was attributed to cross flow generated by the corner 
interaction, which extends well beyond the influence of the corner shocks and is not included in the hypothesis of Babinsky et al. [10]. Spanwise flow diverted by the upstream corner separation towards the tunnel centreline has also been experimentally measured by Délery [43], Eagle and Driscoll [51], Helmer et al. [69]. These authors suggest that this lateral velocity is the mechanism responsible for three-dimensional effects in ducts of very small viscous aspect ratios $[11,51]$.

Benek et al. [12] modelled corner flows with the Quadratic Constitutive Relation (QCR) terms proposed by Spalart [119]. Accounting for compressibility and anisotropy effects, these terms are thought to be responsible for the generation of secondary corner flows and have been shown to improve agreement with the experimentally measured flowfields in rectangular wind tunnels $[36,84]$. For a heavily separated oblique shock reflection in a confined tunnel of $w / h=0.25$, the computed surface streamlines on the channel floor are presented in figure 2.21. It can be seen that the pattern simulated by the turbulence-model with QCR terms (figure 2.21b) is more complex than that without QCR terms (figure 2.21a). The existence of additional critical points observed in figure $2.21 \mathrm{~b}$ was explained by the corner vortices generated by the QCR terms, which act to increase the communication between the corner separations and the central flow. This investigation showed that prediction of SBLI flow fields can vary wildly with the use of different turbulence models. This finding also holds true for the results of Bruce et al. [24], where six RANS models produced varied sizes and structures of corner interactions in a normal SBLI. It has been concluded by multiple

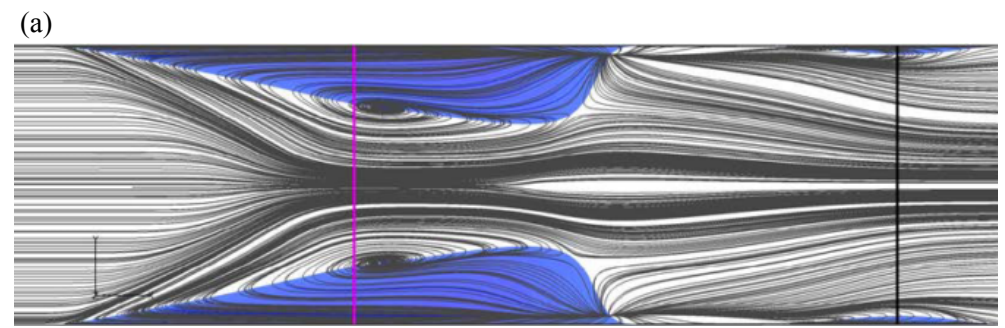

(b)

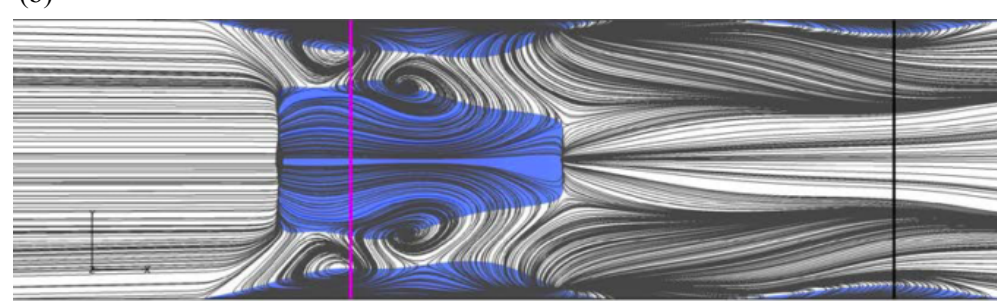

Fig. 2.21 Simulated oil-flow visualisation on the tunnel floor for a strong interaction at $M_{\infty}=2.9$ in a small-aspect-ratio channel of $w / h=0.25:(a)$ without the QCR terms; $(b)$ with the QCR terms. Blue shaded region indicates separation, from [12]. 
authors $[11,12,24]$ that the simulation capability for SBLIs, especially into corner flows, is not yet sufficiently accurate to confidently predict corner effects.

In summary, for SBLIs in a straight channel, it has been shown that flow around the mid-span region can be strongly affected by the state of the flow in the corners and on the sidewalls. A number of researchers have tried to scale the extent of three-dimensionality or explain the driving mechanisms for corner effects. The existence of compression waves caused by the displacement effect of corner separation appears to be the primary mechanism coupling corner flows and other regions, although secondary cross-flows have also been suggested as a dominant factor. A few computational investigations have supported this hypothesis, however no quality experimental data are available to validate the observations. A deeper concern is that a comprehensive understanding of the role of corner shocks in oblique SBLIs has not yet been reached. The three-dimensional wave structure modified by corner shocks and the impact this has on the local APG remains unanswered.

\subsubsection{Three-dimensional separation flow topologies}

The closed bubble concept defined in two-dimensional separation is inadequate and inappropriate in three-dimensional flow topologies. Critical point theory [80] is a powerful tool for rationally describing flows containing separations in three dimensions. This theory focuses on the skin friction lines on a surface. Construction of the skin-friction-line pattern reveals the existence of critical points, where the skin friction vector vanishes. Critical points can be of the node $(\mathrm{N})$, saddle point $(\mathrm{S})$, or focus $(\mathrm{F})$ type, linked either to an attachment or a separation depending on the behaviour of the skin-friction lines in its vicinity. Within this framework, a flow is said to be separated if the surface flow pattern contains at least one saddle point, through which a separation line passes.

For interpretation of the skin-friction-line patterns, readers are referred to the work of Délery (2013) [44], who has classified a series of three-dimensional separation topologies indicating the spatial structures from the surface traces.

A common feature in three-dimensional separations is the 'owl-face' proposed by Perry and Hornung [104]. Figure 2.22a shows an 'owl-face of the first kind', containing a leading edge saddle point which diverges the upstream streamlines. In this flow several friction lines coming from upstream, and the separation line, spiral into the focus points, which are the surface traces of 'tornado-like' vortices. Fluid then escapes through these vortices, creating an 'open' separation (fluid that leaves the wall does not recirculate along a closed streamline $[135,139])$. To ensure topological consistency, a second saddle point $S_{2}$ exists on the reattachment line. 
(a)

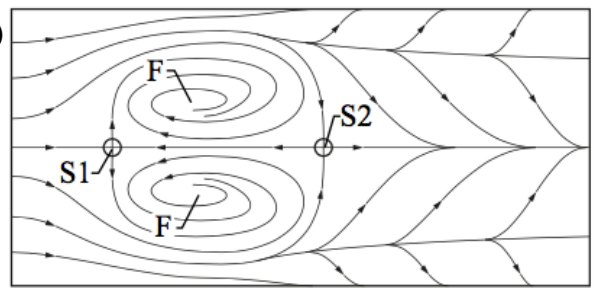

(b)

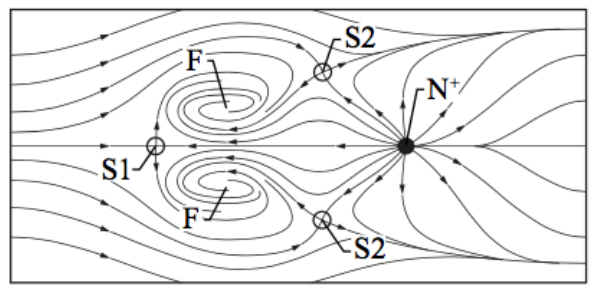

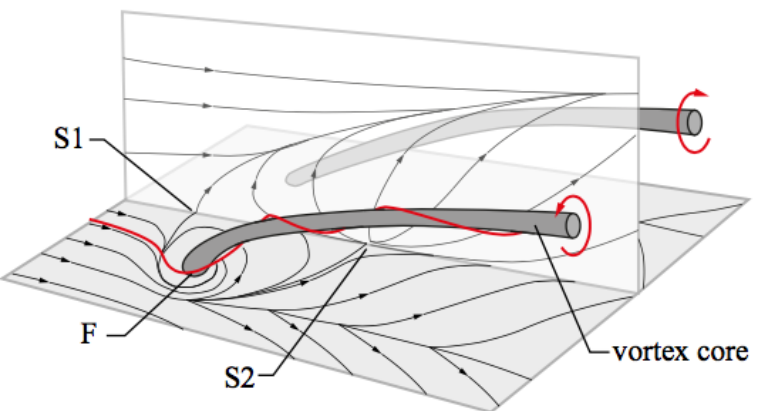

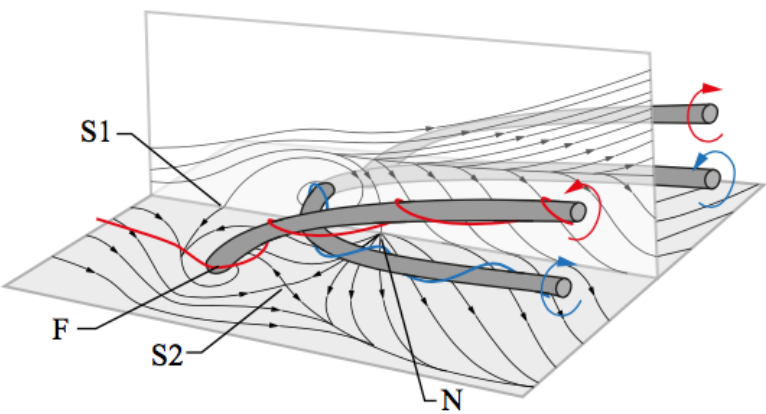

Fig. 2.22 Three-dimensional flow fields associated with flow separation described by Perry and Hornung [104]: (a) owl-face of the first kind; $(b)$ owl-face of the second kind, adapted from Colliss [34].

Shown in figure $2.22 \mathrm{~b}$, the 'owl-face of the second kind' is of a similar, yet more complicated organization. More specifically, the downstream portion differs in the way the rear saddle point is split into two and offset laterally, with an additional node added to build a topologically consistent and physically possible pattern. The effect on the outer flow is to induce an additional horseshoe vortex, which partially offset the upwash around the centre-span produced by the more widely spaced primary vortices.

In an experimental investigation on normal SBLIs, Ogawa [98] reported that the shockinduced separation can be classified as an 'owl-face of the first kind' in a $M_{\infty}=1.3$ flow. However for a stronger interaction with $M_{\infty}=1.5$, the flow topology was observed to transit into the more complicated second kind. In a study of SBLI control [34], the shape and size of separated regions present significant variations among baseline case and controlled flows. By analysing features of the surface streamline-patterns, the author attempted to link flow topologies to the likely performance of control devices.

For SBLI flows, different separation topologies are seen to be dependent on the shock strength and separation size. Examination of surface flows can help to determine the threedimensional spatial structures and the presence of vortices, which may have a more global effect on flow field. 


\subsection{Flow control}

Section 2.3 explained that the corner separation can couple with the main interaction as observed around the centre-span of a channel wall, with the result of significantly increasing the three-dimensionality of the overall flow field. Therefore, flow control employed in the corner regions might have the potential to not only affect the local separation but also affect the global flowfield. However many of the existing control studies in wind tunnel facilities have focused on the centreline flow. Very few experimentalists have attempted to control corner interactions, and their results will be discussed here.

Boundary-layer control modifies the viscous near-wall flow, and aims to prevent shockinduced separation [7]. This control method can take many forms, with recent reviews of this subject given by Ashill et al. [5], Babinsky and Ogawa [9]. Here only two of the most commonly used types, namely active suction and vortex generators (VGs), are explored.

\subsubsection{Boundary layer suction}

The fundamental principal of suction (also termed bleed) is to remove the low-momentum fluid contained within the near-wall region, hence increasing a boundary layer's resistance to separation, as shown in figure 2.23. However, with increasing suction strength, the additional drag associated with bleed also goes up, which in turn reduces the suction effectiveness [13].

For studies of this control method in oblique SBLIs, no agreed optimum location for suction has been reached. In an experimental investigation at $M_{\infty}=2.5$, Fukuda et al. [54] found that bleeding downstream of the interaction was best for preventing separation. Sun

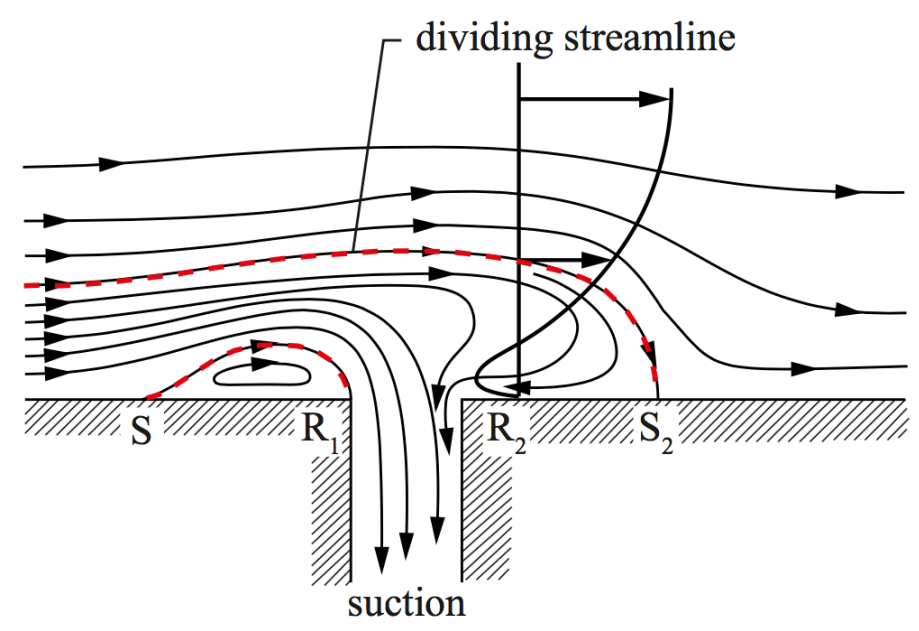

Fig. 2.23 Schematic representation of the flow in a separated bubble with suction applied, from Délery and Bur [40]. 
and Childs [122] conducted a series of experiments, with Mach numbers varying from 2 to 4. They reported that suction was best placed across the interaction, provided the bleed pressure was low enough to avoid recirculation within the plenum chamber. This is in agreement with the finding of Seebaugh and Childs [113], who suggested that removal of approximately 3\% of the boundary-layer mass flow from the interaction region can eliminate separation in an axially symmetric (i.e. two-dimensional) flow. Other studies, for example Hingst and Tanji [72], noted that upstream bleed was as capable at controlling oblique SBLIs as suction within the interaction domain. Compared with bleed applied to normal interactions, it is generally more difficult to achieve the required suction mass flow rate in supersonic flows because of the lower surface pressures.

Titchener et al. [130] examined the effect of corner bleed applied to a normal SBLI. Tangential slots $\left(60^{\circ}\right)$ in the corner regions were placed approximately 24 incoming boundarylayer thicknesses upstream of the normal shock. A bleed mass-flow rate as small as $0.2 \%$ of the tunnel mass flow (approximately $7 \%$ of the boundary-layer flow) was found to effectively suppress the corner separations. The prominent corner effects, otherwise observed in uncontrolled flow, were therefore mitigated, and the three-dimensionality of the overall flow field was considerably reduced. The suppressed corner interaction however led to an increased centreline separation, resulting in higher total pressure losses in the central region downstream of the interaction.

In a further study by Titchener and Babinsky [127], it was observed that when flow control was only introduced to the tunnel centre-span, the local separation was delayed whilst corner effects were enhanced. The overall losses therefore tended to be redistributed instead of removed. In the case where corner bleed was employed in tandem with a central-flow control, a significant flow improvement was obtained. This interplay between the corner and centreline separation is consistent with the results of the studies presented in section 2.3.2, confirming that manipulating corner flows can significantly alter the nature of the main SBLI.

Although boundary-layer suction has been successfully used to mitigate the corner effects, this technique also reduces the overall mass flow, which can be detrimental in some situations. In addition, a sub-skin plenum chamber is required for the suction system. For engineering applications such as aircraft engine inlets, these drawbacks can result in added weight and drag. Vortex generators have therefore been studied as an alternative control technique to reduce the requirement for suction. 


\subsubsection{Vortex generators}

Vortex generators act to induce stream-wise vorticity, which can energize the boundary layer by entraining high-momentum flow from further away from the surface towards the wall. Such devices have successfully demonstrated their abilities at SBLI control and continues to be one of the most widely researched techniques, as highlighted by the reviews of Ashill et al. [5], Lin [81], Nolan [97].

VGs can be classed according to their size: traditional VGs are normally sized to be slightly greater than the local boundary layer thickness; while micro-VGs typically have heights between $10 \%-50 \% \delta$, extending beyond the viscous sublayer [81]. The more recently developed micro-VGs introduce vortices embedded inside the boundary layer and redistribute momentum internally, promising similar benefits to traditional VGs but at a reduced device drag [9]. A selection of designs are shown in figure 2.24. Among these geometries, micro-vanes are generally observed to be most effective at reducing shockinduced separations $[8,73,123]$.

(a)

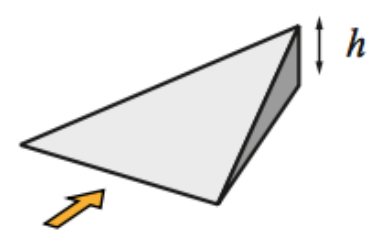

(b)

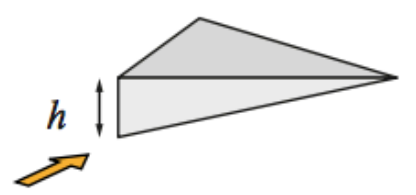

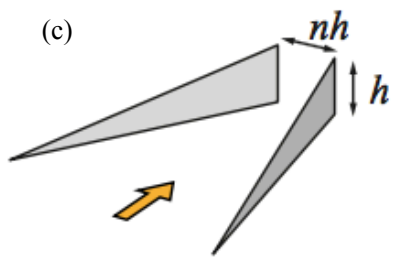

(d)

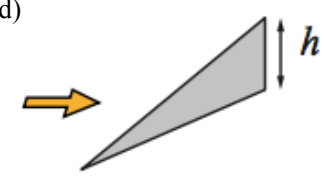

Fig. 2.24 Types of micro-vortex generators, adapted from [81]: $(a)$ forwards wedge; $(b)$ backwards wedge; $(c)$ counter-rotating vanes; $(d)$ single rotating vane. Height $h$ is the key parameter.

The circulation of a vortex $\Gamma$ measures its vortical strength and is considered the most fundamental parameter charactering VG performance. Following [5], $\Gamma$ can take the functional form:

$$
\frac{\Gamma}{u_{\tau} h}=G\left(h^{+}, \frac{x-x_{t}}{h}\right)
$$


where $h^{+}=u_{\tau} h / v$ is the height-based device Reynolds number and $x_{t}$ is the streamwise location of the trailing edge of the VG. The relations described by the function $\mathrm{G}$ are shown in figure 2.25. In figure 2.25a, the strengths of different shaped VGs are seen to collapse nicely onto a single curve when the devices are scaled to an 'effective height' $h_{\text {eff }}$. The measured circulation has been found to be proportional to the device height and $u_{\tau}$ above a critical Reynolds number. For smaller VGs below this critical Reynolds number (in the micro-VG size range), there is a pronounced drop-off in circulation production. Figure $2.25 \mathrm{~b}$ illustrates the decay of vortex strength downstream of a device, suggesting that the effectiveness of VGs is very sensitive to their relative position to the flow control region. Equation (2.2) indicates that micro-devices should be placed closer to adverse pressure gradients compared to the traditional VGs, and that the optimum location is likely to be a function of device height [7].

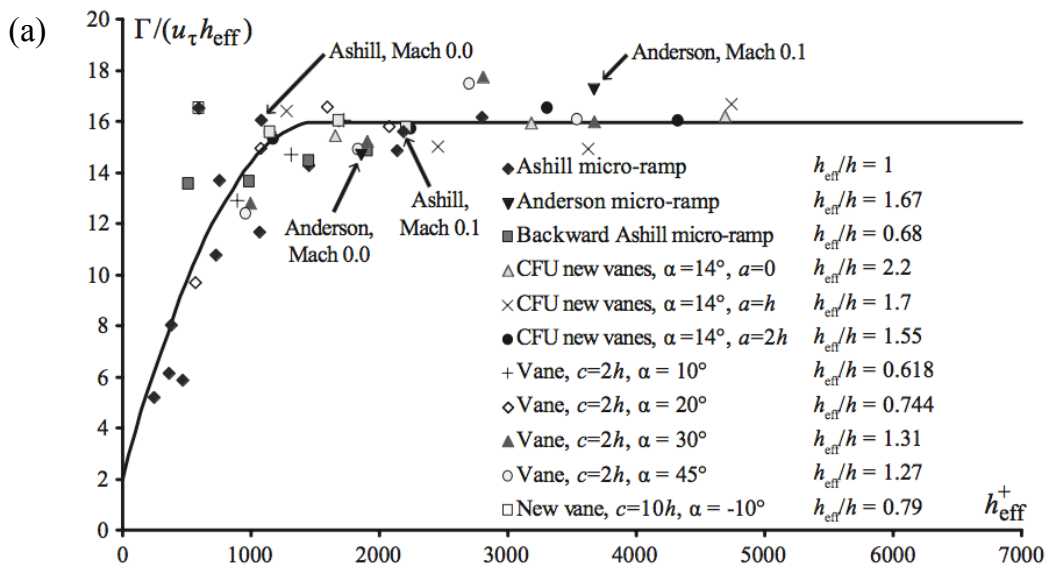

(b)

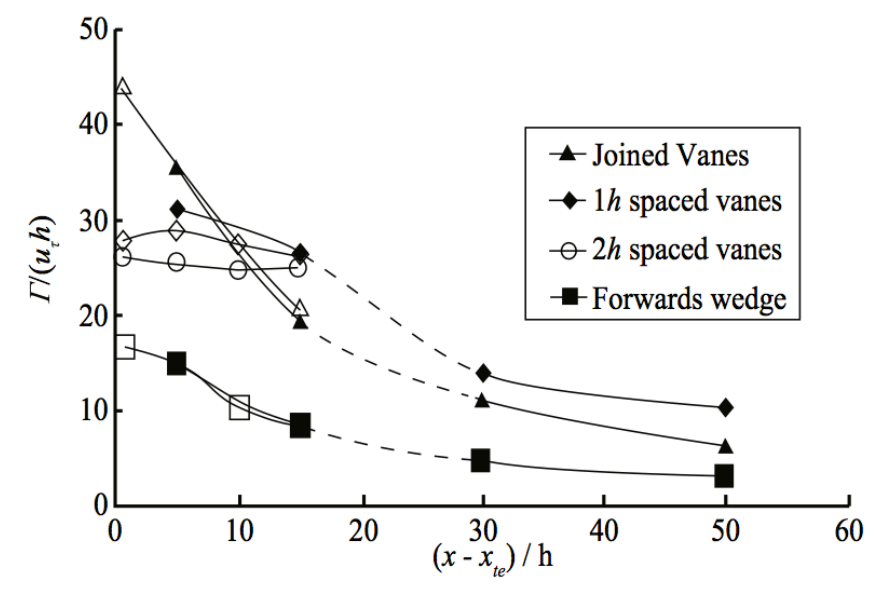

Fig. 2.25 Variation of non-dimensional vortex strength with: (a) effective height $h_{\text {eff }}^{+}$, from Nolan [97]; (b) downstream distance, from Ashill et al. [5].

Much success has been reported when VGs are applied to a normal SBLI [30, 73, 93, 128 , 129]. In an experimental study at $M_{\infty}=1.5$ [73], micro-vanes placed ahead of an normal 
interaction completely eliminated the shock-induced separation. However, VGs have shown to be generally less effective at controlling oblique SBLIs. Babinsky et al. [8] conducted experiments at $M_{\infty}=2.5$ to investigate the ability of micro-VGs to delay separation in a reflected shock interaction. With an array of micro-ramps introduced to the central region of the tunnel floor, a fully-separated uncontrolled flow was broken into a series of individual 'cells' of three-dimensional separation. This cellular structure of separation was also observed by Giepman [60]. Based on PIV measurements, Blinde et al. [14] explained the fragmentation phenomenon as a redistribution of separated regions. More recent work by Nolan [97] has noted a tendency for the circulation to decrease with increasing Mach numbers, especially for the higher speeds tested $\left(M_{\infty}=2.5\right)$. This Mach number effect can be a possible explanation for the reduced effectiveness of VGs in the control of oblique interactions.

Although their ability to eradicate or delay the centreline separation has been confirmed, micro-VGs have shown to be unpredictable and often unfavourable at controlling corner flow. In a study of corner effects for normal SBLIs, Sami [110] introduced vanes near the corners with the original aim to prevent local flow separation. However the opposite effect was seen to occur. Similar results have been reported by a number of other studies [23, 25, 28, 52], where the application of corner VGs resulted in an increased size of corner separations (see the surface flow visualisation from [25] in figure 2.17).

Refinement of independent micro-VG systems for effective corner flow control merits further attention. The benefits of combining bleed and vortex generators also continue to be an area for active research. 


\subsection{Summary and research aims}

\subsubsection{Key remaining questions in corner effects for oblique SBLIs}

The physics of nominally two-dimensional SBLIs have been studied for many decades and are mostly well-understood. When such interactions occur in rectangular channels, which is the most common geometry for such investigations, the interaction of the shock wave with the corner flow results in a complicated three-dimensional SBLI in the near-wall regions. The approach often taken by previous studies is to discount the presence of corners and focus measurements along the central region of the channel floor, assuming that flow conditions well away from the sidewalls are more or less two-dimensional. However, more recently it has been noted that any flow separation occurring in the streamwise corners can significantly change the overall flowfield. This is especially true for facilities of large "viscous aspect ratios' [25]. In such a scenario, erroneous results can arise for SBLI-investigations examining centreline flow independent of corner separations.

An appreciation of the corner separation is required to determine the actual physics governing the interactions within a rectangular duct. However, the literature review indicates that the understanding of the sidewall effects remains very nascent. In the transonic normal SBLI, previous studies have found that the coupling between the corner separation and centreline separation is mainly achieved through the so-called corner shocks altering the APG experienced by the flow elsewhere. These corner shocks arise from the displacement effect of the corner separation, which generally occurs well upstream of any separation around the central regions of the channel walls.

For the case of a supersonic oblique shock-wave reflection interacting with the boundary layer on the floor of a channel, a similar mechanism has been proposed. Secondary lateral flows induced by corner separations have also been suggested to be a factor responsible for sidewall effects. While a number of computational investigations have tended to focus on the flow behaviour with corner effects present, a comprehensive understanding of the underlying flow physics, and the role of corner shocks in particular, has not yet been achieved. There appears to be no clarity on the exact structure of the wave pattern induced by corner separations, and how (if at all) the corner waves modify the two-dimensional oblique SBLI, and the APG experienced by the floor boundary layer.

Furthermore, the literature review has pointed to the inconsistencies in the surface flow topologies produced by different numerical models, suggesting that the simulation capability into corner flow modelling is not yet sufficiently accurate. However, little validation quality data is available for an oblique SBLI in a straight channel. An examination into existing SBLI control studies shows that future effort is required to effectively reduce corner effects in an 
oblique reflecting interaction. There have been no experimental investigations systematically altering the size of the corner separations for an established oblique SBLI in order to determine the impact of such modifications.

\subsubsection{Research aims and approach}

A number of the questions listed above will be addressed using experimental techniques. The purpose of this study is to extend the current state of knowledge on the three-dimensional flow structures induced by sidewall effects and their effect on the flow elsewhere for an oblique SBLI. The aim is to determine how the relative magnitude of corner separation modifies the size and shape of 'quasi-two-dimensional' separation observed in the centre of a duct and by what physical mechanisms this is achieved. A further objective is to determine the surface flow topology of the centreline separation underneath the reflecting shock wave.

To achieve these goals, experiments are conducted where the corner separations for an established reflecting shock interaction are manipulated to produce corner effects varying in relative size from moderate to significant. The baseline flow conditions are chosen to result in substantial separations both in the corners and along the centreline, which is thought to be a good starting point for this study. 



\section{Chapter 3}

\section{Experimental facility and techniques}

\subsection{CUED No.1 supersonic wind tunnel}

Experiments have been conducted in the supersonic wind tunnel No.1 in the Cambridge University Aerodynamics Laboratory. In figure 3.1 the general layout of the system is shown by a schematic diagram and a photograph. This tunnel is of the intermittent blow-down type fed from a high-pressure reservoir of dry air and the flow exhausts to atmosphere. The

(a)

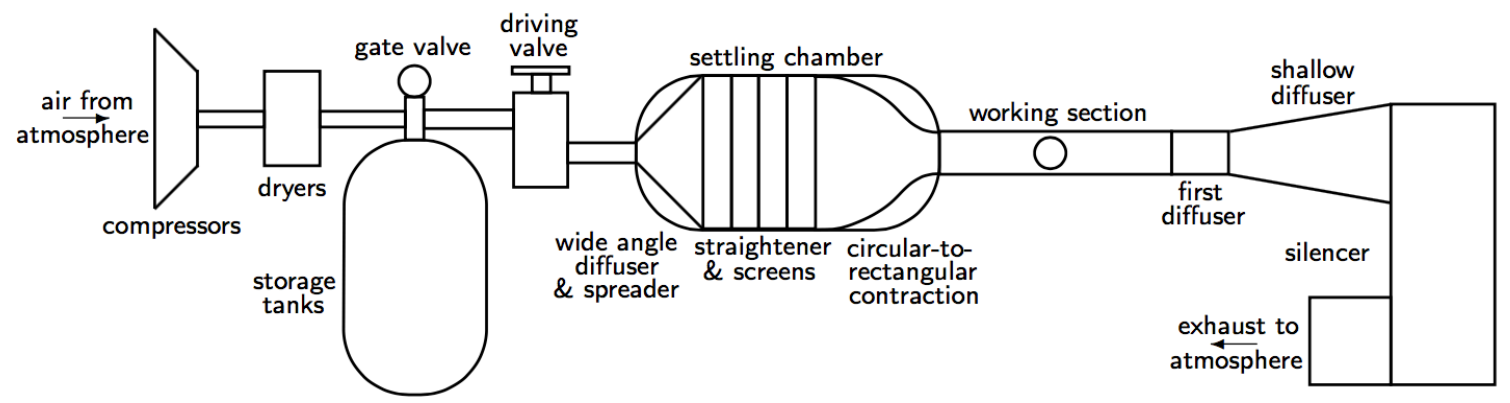

(b)

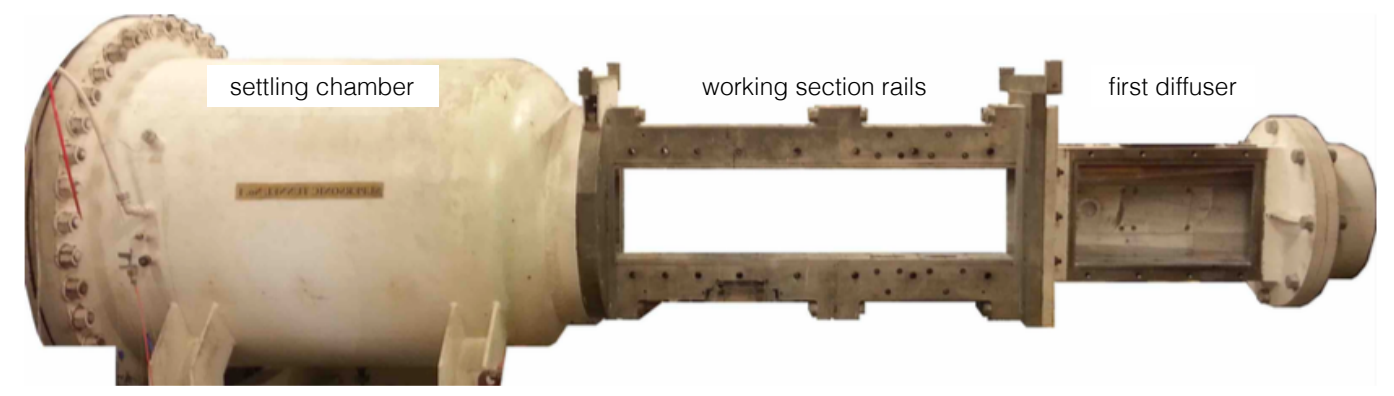

Fig. 3.1 CUED No.1 Supersonic wind tunnel: $(a)$ schematic illustration; $(b)$ photograph of the tunnel without doors and nozzles, adapted from [37]. 
mass flow rate is manually controlled by a pressure regulator and this maintains a relatively constant stagnation pressure fluctuating by about $0.5 \%$ during a typical run.

The air then passes through a wide angle diffuser into the settling chamber, where an array of fine mesh screens and honey-comb straighteners are used to calm the flow. A contraction of ratio 18 follows to moderately accelerate the flow to subsonic speeds before entering the working section, where an interchangeable convergent-divergent nozzle is set to achieve the required Mach number. For this study, the nozzle geometry is fixed to an inflow $M$ of 2.5 for all experiments, with a unit Reynolds number of $40 \times 10^{6} \mathrm{~m}^{-1}$.

The working section is of rectangular cross-section, $114 \mathrm{~mm}$ wide by $178 \mathrm{~mm}$ high, where the experimental rig is mounted through the top and bottom rails seen in figure $3.1 \mathrm{~b}$. The sidewalls are removable doors which provide optical access with different windows: $457 \mathrm{~mm} \times 203 \mathrm{~mm}$ rectangular windows for stagnation pressures below approximately $200 \mathrm{kPa}$, while smaller circular windows of a $203 \mathrm{~mm}$ diameter for higher working pressures. The sides of the working section are bolted to the rails with sealing strips applied to prevent leaks.

The working section is designed to incorporate an ejector system for boundary layer bleed. On top of the suction plenum chamber, the tunnel floor can be replaced by an aluminium plate, allowing bleed of certain hole/slot arrangements to be located at where suction is required. Driven by the same high-pressure air as the wind tunnel working section, the ejector system can nevertheless be adjusted independently and maintain a constant mass flow through the slots. The maximum mass flow rate achievable by the suction system is $0.5 \mathrm{~kg} / \mathrm{s}$ with the tunnel off. In the present study bleed in the form of corner slots is employed to reduce three-dimensional effects, with details given in section 3.2.1.

\subsubsection{Set-up}

Set-up in the working section for current investigation is shown in figure 3.2. The stagnation pressure is set to $380 \mathrm{kPa}$, which is the minimum settling chamber pressure to avoid unstart hence stably locate the incident shock. The stagnation temperature measured by thermocouples in the settling chamber is set at $296 \mathrm{~K} \pm 3 \mathrm{~K}$. The variation of stagnation temperature depends on the season (higher room temperature increases $T_{0}$ ) and the time of day ( $T_{0}$ is typically $4 \mathrm{~K}$ higher after a day of running). During the steady flow period of a tunnel run, $T_{0}$ is observed to increase at a reasonably consistent rate of $0.07 \sim 0.1 \mathrm{Ks}^{-1}$, giving a maximum variation of $7 \mathrm{~K}$. It can be seen that a half-liner configuration is used here. By blocking the lower half of the tunnel, the streamwise distance for observation through the sidewall circular window is almost doubled and the possible run time is increased to the order of 1 minute. The resulting test section is $114 \mathrm{~mm}$ wide and $86 \mathrm{~mm}$ tall. 

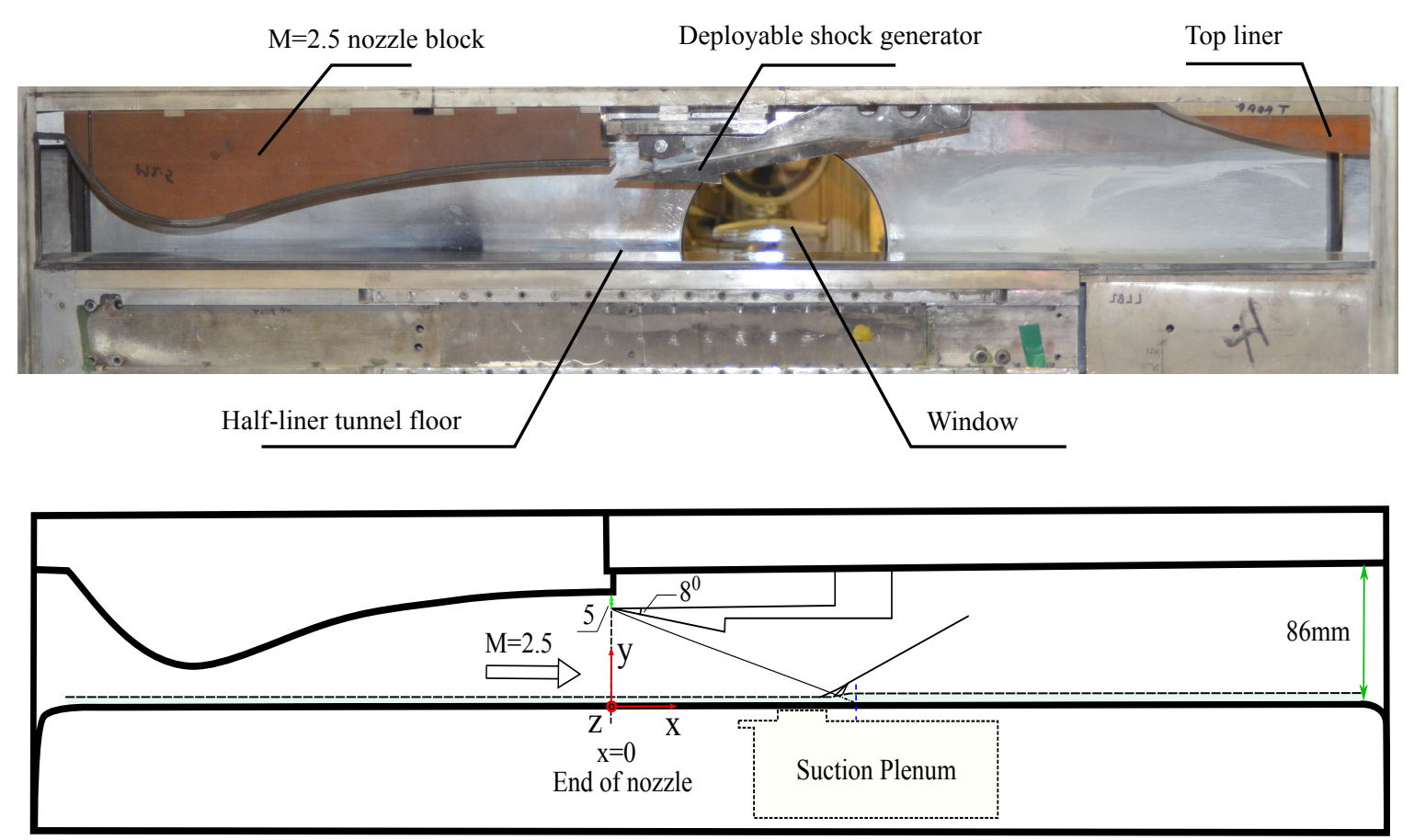

Fig. 3.2 Setup for the working section.

A deployable wedge on the ceiling of the tunnel is lowered to $8^{\circ}$ to generate an oblique shock once the desired supersonic flow is developed. In $M=2.5$ flow such a wedge angle can result in an oblique SBLI with substantial shock-induced separations and the interaction domain is positioned within the visualisation area through the window, which correspond to the flow conditions required for baseline experiment. A gap of $5 \mathrm{~mm}$ between the leading edge of the deployed shock-generator and the upper surface of the tunnel enables the boundary layer on the nozzle block to disappear into gap and therefore creates a 'cleaner' environment for the generation of the incident shock. At the exit of the working section a top liner is bolted on the tunnel ceiling to allow a smooth geometric transition to the first diffuser. The structure at the joint of the working section and diffuser system is hence more aerodynamic and characterizes with a lower total-pressure losses.

A coordinate system following convention is used where $x$ refers to the streamwise direction with $x=0 \mathrm{~mm}$ corresponding to the end of the nozzle. The theoretical inviscid shock reflection location calculated from the leading edge of the deployed wedge to the tunnel floor $(78 \mathrm{~mm})$ and shock angle $\left(31.01^{\circ}\right.$ for $8^{\circ}$ flow turning) is at $x=135 \mathrm{~mm} . y$ is measured vertically upwards with $y=0 \mathrm{~mm}$ referring to the tunnel floor. Spanwise position is denoted by $z$, measuring from the centreline of the tunnel floor with the sidewalls being at $z= \pm 57 \mathrm{~mm}$. 


\subsection{Corner flow manipulation}

To examine how changes in the corner flow field can affect the main oblique SBLI, modifications to the corner geometry are applied to change the onset and magnitude of corner separations.

\subsubsection{Corner-control modification}

Corner suction and micro-vortex generators are introduced to study the effect of a reduced corner separation, with the arrangements depicted in figure 3.3.

(a)

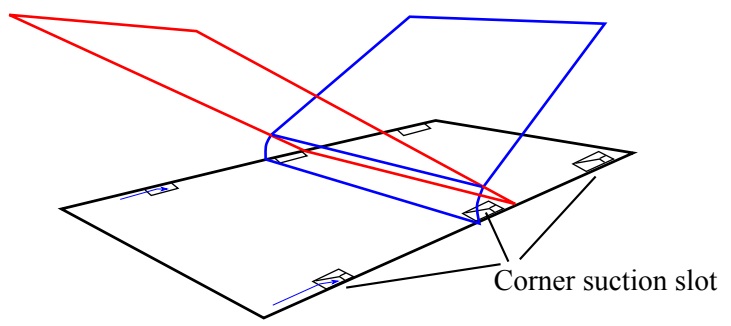

(b)

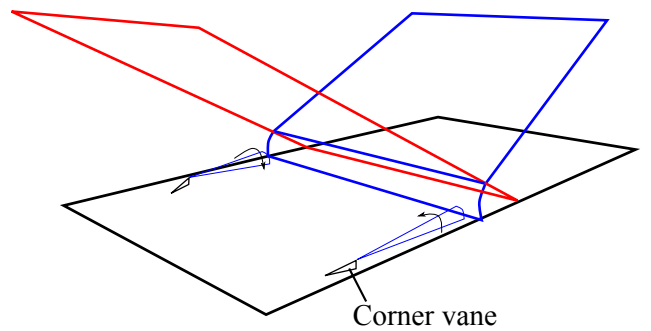

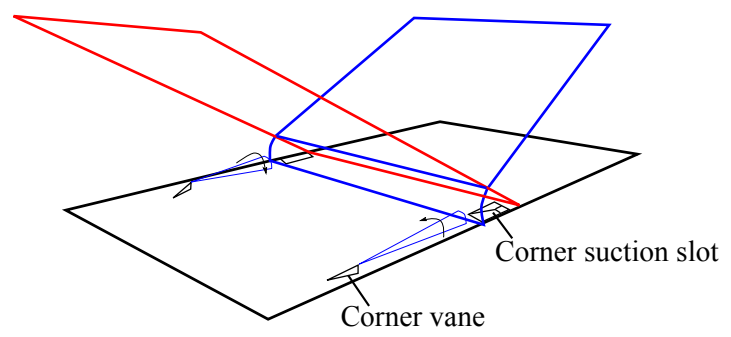

(c)

Fig. 3.3 Experimental arrangements of corner flow control.

In the case of active suction, previous studies on supersonic interactions have shown no agreement on the optimum bleed position for boundary layer control. The corner slots are hence placed upstream, across and downstream of the interaction, with the leading edge of the slots located at $x_{\text {slot }}=20,100,150 \mathrm{~mm}$ respectively (as show in figure $3.3 \mathrm{a}$ ).

A schematic diagram of the suction plate with key dimensions is provided in figure 3.4. The tunnel floor above the suction plenum consists of an interchangeable plate covering an $180 \mathrm{~mm}$ long region of the test section with the leading edge located $18 \mathrm{~mm}$ downstream of the nozzle end. The plate is $10 \mathrm{~mm}$ thick, which is suggested by Colliss [34] to be sufficient to reduce any thermal influence on the accuracy of pressure sensitive paint technique. Three suction plates are used with the tangential corner slots cut into them at designed bleed 
Plan view

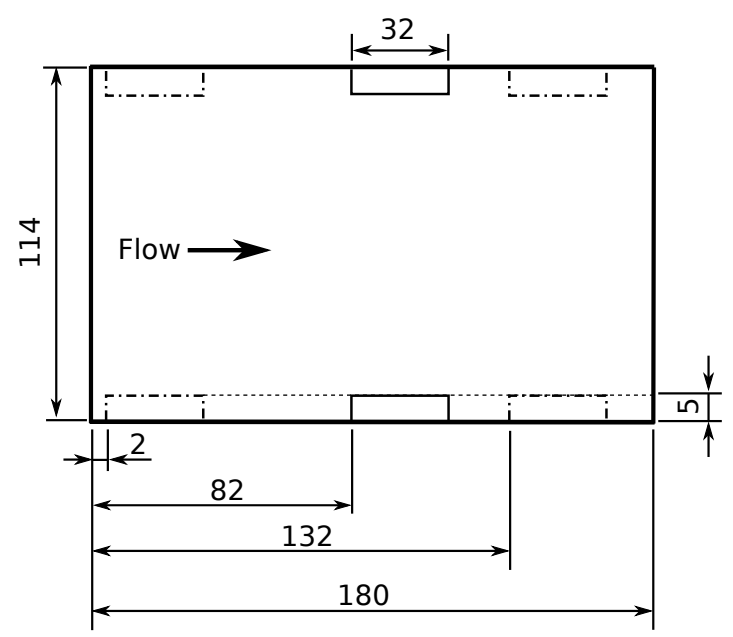

Side view

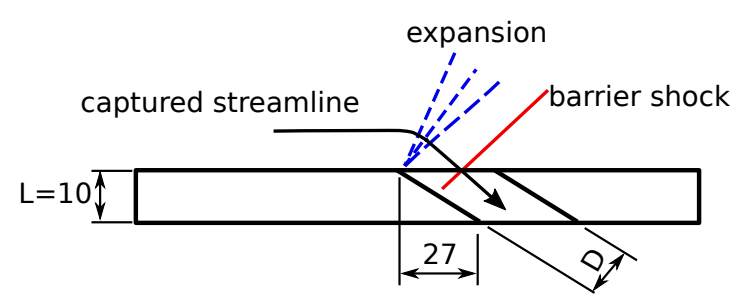

Fig. 3.4 Suction plate diagram of corner suction (dimensions in $\mathrm{mm}$ ).

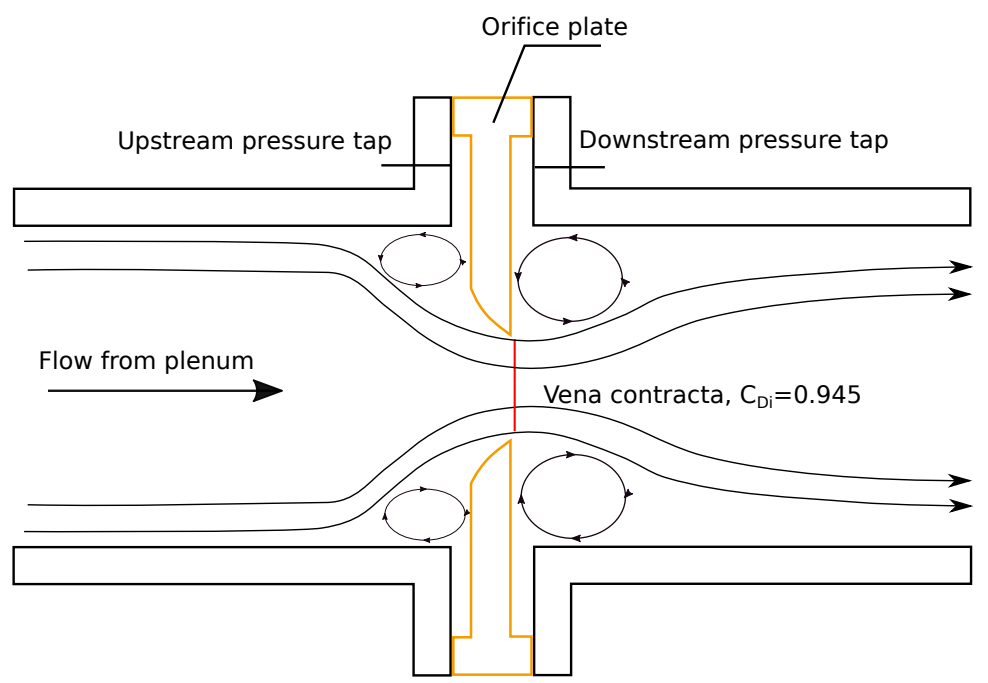

Fig. 3.5 Schematic diagram of flow pattern through a quarter-circle orifice plate.

positions. The slot is $32 \mathrm{~mm}$ long by $5 \mathrm{~mm}$ wide, with the angle set to be $20^{\circ}$ as shown in figure 3.4b. In an analytical model proposed by Harloff and Smith [66], it has been shown that $20^{\circ}$ slots relative to the local surface can minimize bleed drag in supersonic flow. This study also stated that slots with an angle less than $50^{\circ}$ and a $L / D \leqslant 3$ can achieve larger mass flow rate due to an absence of internal-hole flow separations. An experimental study by Willis et al. [138] confirmed that $20^{\circ}$ tangential slots were characterized with higher bleed efficiency than normal holes. For such a configuration an expansion fan is created to turn the 
low-momentum fluid into suction slots, while a normal shock (termed barrier shock) follows to return the flow back to the streamwise direction [15].

The mass flow rate through the corner suction is set by a $12.5 \mathrm{~mm}$ diameter quarter-circle orifice plate between the plenum chamber and the ejector, as shown in figure 3.5. Two static taps located upstream $\left(P_{\text {upstream }}\right)$ and downstream $\left(P_{\text {downstream }}\right)$ of the orifice plate allow the measurement of the pressure ratio $\frac{P_{\text {downstream }}}{P_{\text {upstream }}}$ to verify whether flow through the orifice is supersonic downstream $\left(\frac{P_{\text {dowsntream }}}{P_{\text {upstream }}}<0.5238\right)$ and thus choked $(M=1)$ at the effective throat. Using $P_{\text {upstream }}$ to determine the stagnation pressure at the orifice, the mass flow function can be applied:

$$
\dot{m}=1.281 \frac{C_{D i} A_{\text {orifice }} P_{\text {upstream }}}{\sqrt{c_{p} T_{0}}}
$$

However, the area of actual jet throat (termed Vena Contracta), is smaller compared to $A_{\text {orifice }}$ (the area of orifice) due to streamline curvature. $C_{D i}$, the discharge coefficient, is hence introduced and valued 0.945 to correct for compressible flow through the quarter-circle type orifice following Bragg [20]. The overall mass flow rate into the working section is maintained at $3.296 \mathrm{~kg} / \mathrm{s}$. The bleed strength and its fraction of the overall tunnel mass flow for various bleed positions are given in table 3.1. Here the low suction mass flow rates are a consequence of the low local surface pressures associated with the high Mach numbers. This is especially true for the case of $x_{\text {slot }}=20 \mathrm{~mm}$, where the corner bleed is located upstream of the incident oblique shock.

Table 3.1 Suction strength quantities for corner-bleed tests.

\begin{tabular}{lccc}
\hline Configuration & $x_{\text {slot }}=20 \mathrm{~mm}$ & $x_{\text {slot }}=100 \mathrm{~mm}$ & $x_{\text {slot }}=150 \mathrm{~mm}$ \\
\hline$P_{\text {upstream }}(\mathrm{Pa})$ & 25000 & 34500 & 49500 \\
$\dot{m}(\mathrm{~g} / \mathrm{s})$ & 6.8 & 9.3 & 13.4 \\
$\% \dot{m}_{\text {total }}$ & 0.21 & 0.28 & 0.41 \\
\hline
\end{tabular}


An alternative control method to reduce corner separations is through two micro-VGs as illustrated in figure 3.3b. As shown in figure 3.6, vane-type VGs, featuring a high aspect ratio (i.e. chord length $L$ significantly more than the height $h$ ), are used; such a type was found by Godard and Stanislas [61] to create vortices with greater circulation while incurring less drag. The vane height is $3 \mathrm{~mm}$, approximately $50 \%$ of the incoming boundary layer thickness. The trailing edges of the micro-VGs are placed at $x_{v g}=50 \mathrm{~mm}$, making the non-dimensional distance between the inviscid shock reflection location and the VG trailing edge roughly 28 device heights. Such a streamwise placement is chosen following the study by Babinsky et al. [8], which is shown to enable the beneficial vortical structure remaining in the boundary layers at interaction; meanwhile not too close to break and re-distribute the shock-induced separation into a more three-dimensional pattern. Pearcey [102] reported that vane angles in the range of $16 \sim 20^{\circ}$ were most effective for treating separation. An angle of $15^{\circ}$ is set in current study, which was found by Nolan [97] to produce a peak circulation for vane-type generators.

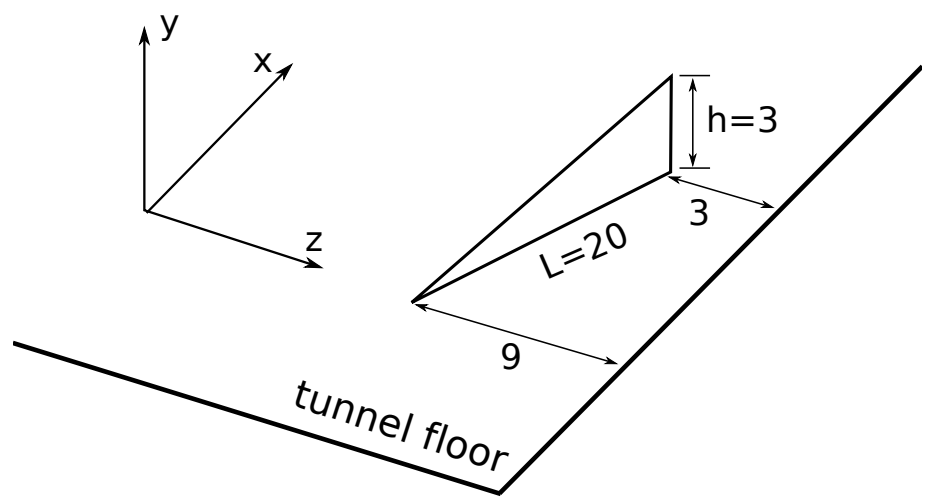

Fig. 3.6 Dimensions of corner vane (in $\mathrm{mm}$ ).

Aside from independent bleed and VG control, the effect of a combined system is also tested. In this set-up shown in figure 3.3c, micro-VGs are placed at the upstream position while bleed slots are located under the VG wake across the interaction. Such a combination can explore whether VGs can reduce the necessary bleed for separation control. 


\subsubsection{Corner-block modification}

As shown in figure 3.7, two obstacles positioned within the corners are utilised to increase the size of corner separations. To achieve different extents of local separation growth, two sets of obstructions, consisting of blocks of dimensions $6 \times 6 \times 20 \mathrm{~mm}^{3}$ and $10 \times 10 \times 20 \mathrm{~mm}^{3}$ respectively, are placed ahead of the inviscid shock location. The streamwise positions of the block front edge range from $x_{\text {block }}=75 \mathrm{~mm}$ to $x_{\text {block }}=130 \mathrm{~mm}$, with a minimum increment of $5 \mathrm{~mm}$ between two consecutive locations.

To gain an insight on the effect of obstacle length, a pair of elongated blockages sized $10 \times 10 \times 40 \mathrm{~mm}^{3}$ is also tested at $x_{\text {block }}=85 \mathrm{~mm}$, allowing a comparison with the flow altered by the standard obstacles.

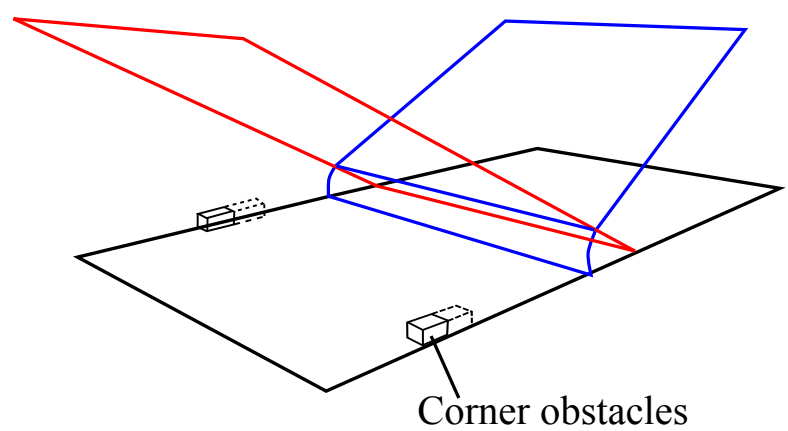

Fig. 3.7 Experimental arrangements of corner obstacles. 


\subsection{Flow visualisation}

\subsubsection{Schlieren imaging}

(a)

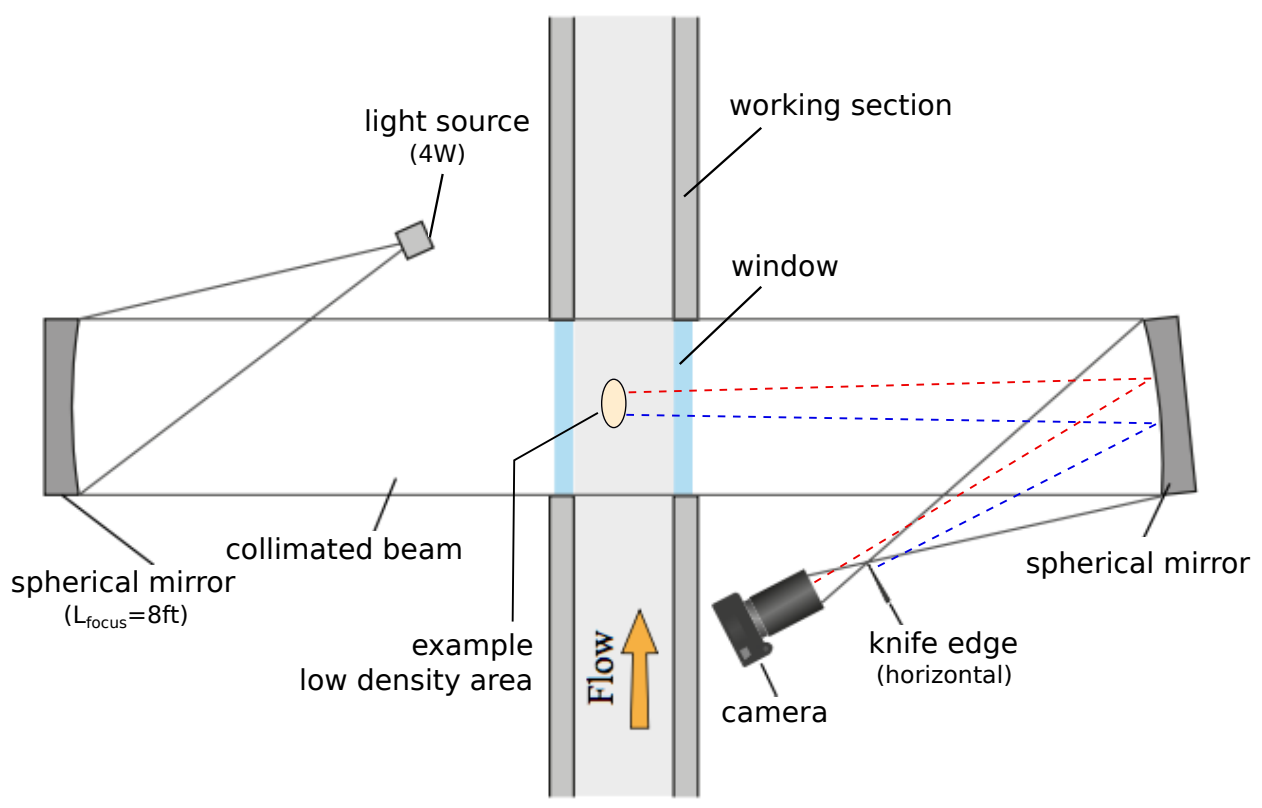

(b)

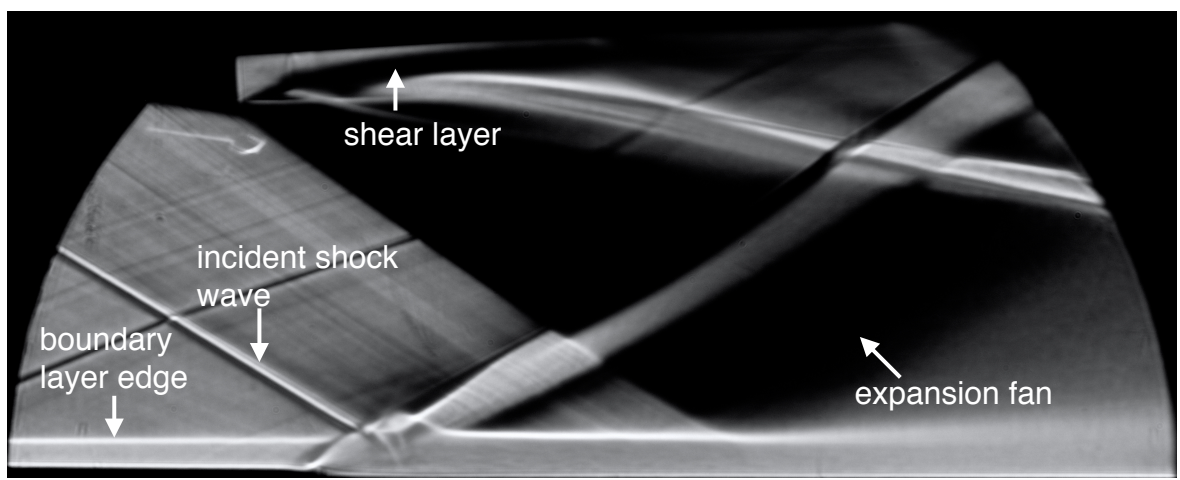

Fig. 3.8 Schlieren visualisation technique: (a) 'z-type' optical set-up; (b) typical result.

Schlieren visualisation is an optical technique widely used for high-speed flow, often as a qualitative technique to develop an intuitive understanding of the general flow field. Relying on the physical principle that air refractive index $\mathrm{n}$ is a function of the fluid density $\rho$ :

$$
n=1+K \rho \quad \text { where } \quad K=0.2274 \mathrm{~cm}^{3} / g
$$


such a technique enables the observation of regions with density gradients due to a variation in the indices of light refraction. Gradients of $\mathrm{n}$ in the direction of light ray propagation $(\mathrm{z}$ for this thesis) do not lead to light refraction, while for the two directions lateral to light the deflection angle $\varepsilon$ can be expressed by:

$$
\varepsilon_{x}=\frac{1}{n} \int \frac{\partial n}{\partial x} d z \quad \text { and } \quad \varepsilon_{y}=\frac{1}{n} \int \frac{\partial n}{\partial y} d z
$$

From these relations it can be seen that light rays are bend towards higher-density regions. A two-mirror z-type horizontal system is implemented for current study to visualize such light deflections, with the schematic diagram of the optical set-up sketched in figure 3.8a.

An LED point light source is placed at the focus of a spherical mirror, which produces a collimated beam passing through the window of the working section perpendicularly. The local beam angle changes slightly when light rays propagate through airs of varying densities, before the full beam is collected by a second spherical mirror positioned at the opposite side of the wind tunnel. A Nikon D7000 DSLR with a 180mm lens is then used to capture the refocused light. Here the operation is described by a low density flow area in the test section. Such a region of a relatively low refractive index acts like a diverging lens and deflects light away from the centre. The received image hence shows a dark patch looking much like a 'shadow' of this low-density flow field, and creates what is known as a shadowgraph. In terms of mathematical interpretation, a shadowgraph image illustrates the second derivative of density field. To gain further optical sensitivity, a knife edge is introduced at the focal point of the second spherical mirror before the camera. Depending on the deflection orientation, lights will either be blocked by or bend away from the knife edge, showing up dark or bright features respectively, which now produces a schlieren image with the light intensity related to the first derivative of air density. Only accurate positioning of the knife edge at the focal point allows visualisation of the full image, otherwise part of the view is blocked. For more details of the schlieren imaging technique, refer to Merzkirch [90].

A typical static image is shown in figure $3.8 \mathrm{~b}$. In this research a horizontal knife-edge is employed such that wall-normal density variations can be highlighted. It can be seen that regions of high density-gradients, such as boundary layers, shock waves, expansions and shear layers, are clearly identifiable. As schlieren visualisation is an effectively instantaneous technique, live videos are also extensively recorded to monitor the establishment of flow field, hence enabling required operations (such as deploying the shock-wedge, applying suction) at a certain stage. 
Despite the many advantages of the schlieren technique, there is also a disadvantage closely related to the present investigation. Since schlieren visualisation is susceptible to density variations through the whole spanwise light path, the resultant image is an 'average' of the three-dimensional flow phenomena (for example corner effects) as the beams integrate across the entire working section width.

\subsubsection{Oil-flow visualisation}

A surface oil-flow technique is used to visualise skin-friction-line patterns on the floor and sidewalls of the working section. This technique relies on a thin layer of oil mixture applied to the surface of interest. During a tunnel run, the oil flow is subjected to the shear stresses exerted by the boundary layers and hence follows the skin-friction vectors. The carrier oil film then evaporates with the colouring agent deposited to map out the local skin friction lines. The resulting oil-flow patterns are photographed once the tunnel runs have finished. A live video is recorded along with the still image to help eradicate any ambiguity caused by mixture smearing upon tunnel shut-down.

For the oil-flow experiments performed in this study, kerosene and finely-powdered titanium dioxide $\left(\mathrm{T}_{i} \mathrm{O}_{2}\right)$ are chosen as the oil medium and pigment respectively, with a $1: 1$ ratio applied. Oleic acid, a dispersant to reduce oil-streak coagulation, is added 1 drop per $10 \mathrm{~g}$ of the kerosene $/ T_{i} \mathrm{O}_{2}$ mixture. As suggested by Brown and Naughton [22], a high-viscosity oil film can help to reduce the sweeping-back effects by shutting down, a small amount of lubricating oil, approximately $10 \%$, is used to add viscosity. The high-viscosity oil layer requires considerable tunnel run times to dry, and thus the maximum run time of 1 minute is used for a typical oil-flow test.

To maximise the quality of the oil streak images, the tunnel floor is sprayed matt black to improve contrast and visibility. High-resolution images are taken with a Nikon D7100, with two spot lights providing an even illumination to the working section. After perspective distortion correction, the picture is used to infer the existence of critical points, determine the locations of separation and reattachment and hence construct the surface flow topology. Interpretation of the surface flow pattern is based on the descriptive introduction by Délery [44] and the work of Perry and Hornung [104], who proposed a series of three-dimensional separation topologies and indicated the structure in space from its trace on the wall.

While the oil mixture follows the surface streamlines well and represents the flow topology accurately for attached flow, it has been noted by Squire [120] that an early (i.e. more upstream) indication of separation can occur as the oil tends to stop moving before the surface skin friction vanishes. This was further explained by the fact that pressure forces in the presence of a large adverse pressure gradient (e.g. separation) are no longer negligible 
and can influence the behaviour of the oil-film layer. More specifically, oil streaks can underestimate the distance to separation by up to $5 \%$ of the upstream influence, making the error in indicated separation location of the order of 0.2 incoming boundary layer thicknesses (approximately $1.2 \mathrm{~mm}$ ) for this study. Given that this error is relatively small, the lengths of shock-induced separated regions shown in the oil-flow visualisation can therefore still be considered as an accurate estimation of separated sizes and be compared among different experiments with confidence.

\subsection{Pressure measurements}

\subsubsection{Static wall pressure taps}

Static wall pressure measurements are conducted via the use of taps embedded in the wind tunnel floor and sidewall. These drilled holes with an internal diameter of $0.15 \mathrm{~mm}$ are connected by long lengths of neoprene tubing (typically $L>30 \mathrm{~mm}$ ) to a 16 channel differential transducer NetScanner 9116, with the set-up shown in figure 3.9. The readings of Channel 1-15 monitor the local static pressures, which are then normalised by the stagnation pressure from Channel 16 through connection to a wall tap in the settling chamber. The atmospheric pressure is required to compute the absolute pressures for a differential transducer, which is read from a calibrated mercury manometer. A study on an identical set-up by Bruce [23] estimated the response time of the tubing to be limited to pressure fluctuations below approximately $33 \mathrm{~Hz}$. Accordingly, this technique is used exclusively to measure time-averaged wall pressures. In the current investigation, the taps are located upstream of the incident shock to

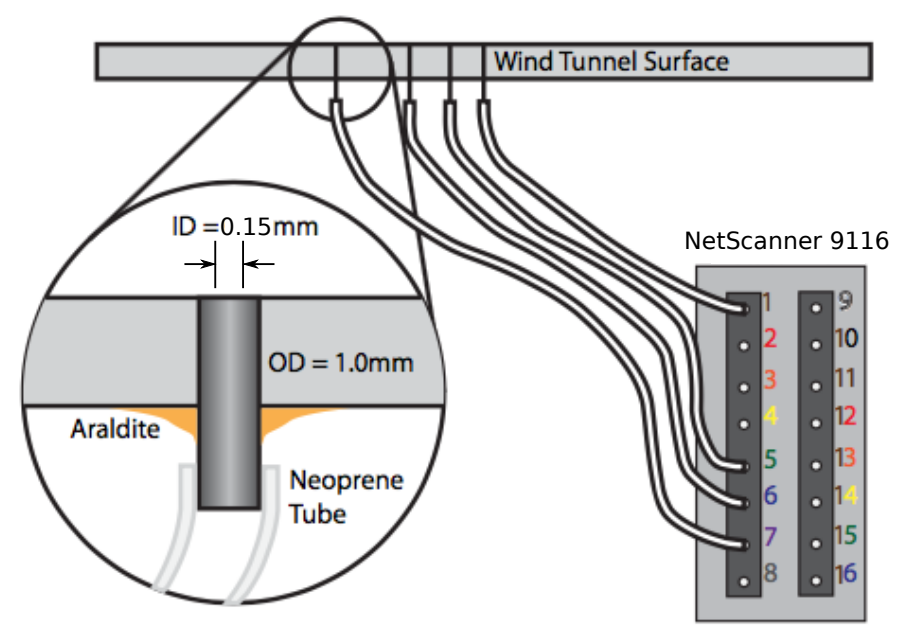

Fig. 3.9 Set-up for static wall pressure measurement, modified from [99]. 
indicate the entry $\mathrm{M}$ and across the interaction domain to calibrate the Pressure Sensitive Paint measurements.

The pressure measured at a static pressure hole is in general greater than the true static pressure. An arithmetical analysis for the solution of the Navier-Stokes equations has suggested that this is caused by a combined effect of deflection of streamlines into the hole and eddies set up in the hole [124]. A further experimental investigation by Shaw [117] showed that the amount of pressure error can be presented as a function of Reynolds number based on hole diameter and skin friction velocity, with the data replotted in figure 3.10. Here the hole length/diameter ratio $(L / d)$ is well above 1.5 , which gives a pressure error up to $2.8 \tau_{w}$, representing an error of at worst $0.5 \%$.

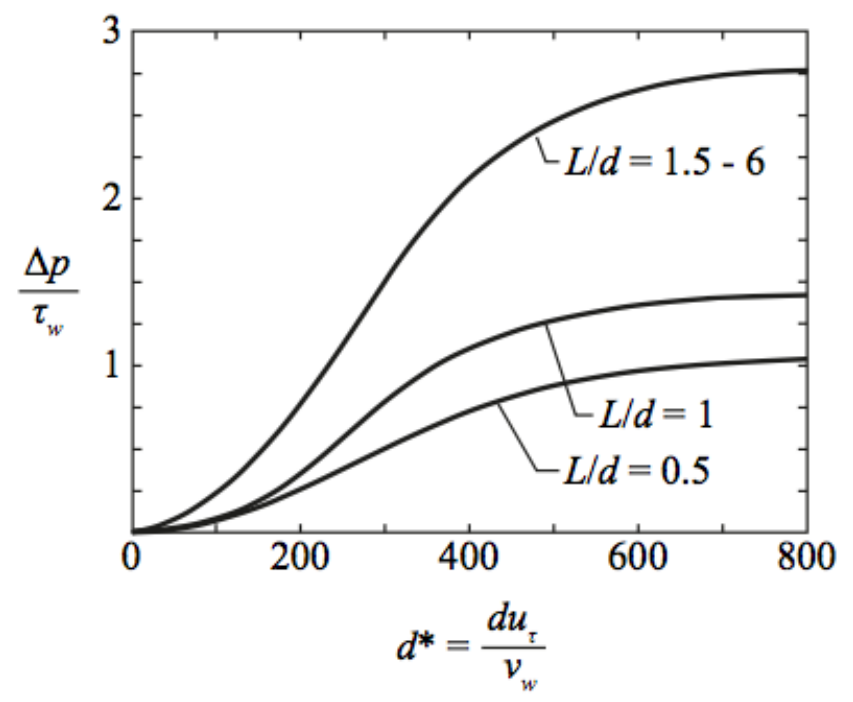

Fig. 3.10 Hole geometry error on the pressure measured by static tap as a function of the Reynolds number based on hole diameter and friction velocity, reproduced from [117].

The NetScanner itself has a manufacturer quoted accuracy of $\pm 0.05 \%$. The mercury manometer can be read to within $\pm 30 \mathrm{~Pa}$, which causes an error of $0.05 \%$ in the atmospheric pressure. The velocity in the settling chamber is estimated to be around $8 \mathrm{~m} / \mathrm{s}$, following Bernoulli's equation an error approximately $0.01 \%$ is introduced. Taking the uncertainties propagation into consideration, the total experimental uncertainty in the static pressure ratio $P / P_{0}$ measured with wall taps is around $\pm 0.5 \%$. 


\subsubsection{Pressure sensitive paint}

The number of pressure measurements that can be obtained with static taps is limited due to the mechanical structure of the tunnel walls. Pressure sensitive paint (PSP) is therefore introduced to provide higher spatial-resolution surface pressure data. The physical principal of this method is illustrated in figure 3.11a.

To apply PSP, the surface of interest is sprayed with a special polymer binder seeded with luminescent molecules (known as luminophore), which is oxygen-permeable. When such paint is irradiated with UV light, luminophores are excited to a higher energy level. Return to their ground state is commenced either by fluorescing in red-shifted light; or by transferring energy to free oxygen molecules to a vibrational mode. The latter reaction is termed oxygen quenching. Higher air pressure makes for oxygen permeation into the binder, leading to a greater concentration of oxygen and hence an increased amount of oxygen quenching, which in turn reduces the intensity of photon emission. Accordingly, luminescent intensity $I$ emitted from PSP is related to the local pressure $P$ in the flow, with the correlation described by the Stern-Volmer equation:

$$
\frac{I_{0}}{I}=1+K_{p} P
$$

where $I_{0}$ is the luminescent intensity in the absence of oxygen quenching (i.e. maximum luminescent value) and $K_{p}$ is the Stern-Volmer constant, [83]. $I_{0}$ is however in general not measurable in wind-tunnel testing, experimental aerodynamicists hence rewrite the function as the ratio to a reference condition:

$$
\frac{I_{r e f}}{I}=A(T)+B(T) \frac{P}{P_{r e f}}
$$

Here the coefficients $A$ and $B$ are related to temperature variation since temperature affects both the oxygen quenching process $(A(T))$ and oxygen diffusion in the polymer binder $(B(T))$. In practice, this temperature dependency is taken into account by comparing the ratio of two pictures: a 'wind-off' image with no air flow and a 'wind-on' image with required flow condition. Additionally, this ratioing also factors out the effects of spatial non-uniformities of UV illumination and coating thickness. Following equation (3.5), $A(T) \& B(T)$, approximating as constants, can then be determined by in-situ static pressure tap calibration.

The set-up for current investigation is shown schematically in figure 3.11b. McLachlan and Bell [87] have found that the linear trend implied by the Stern-Volmer equation is only 
(a)

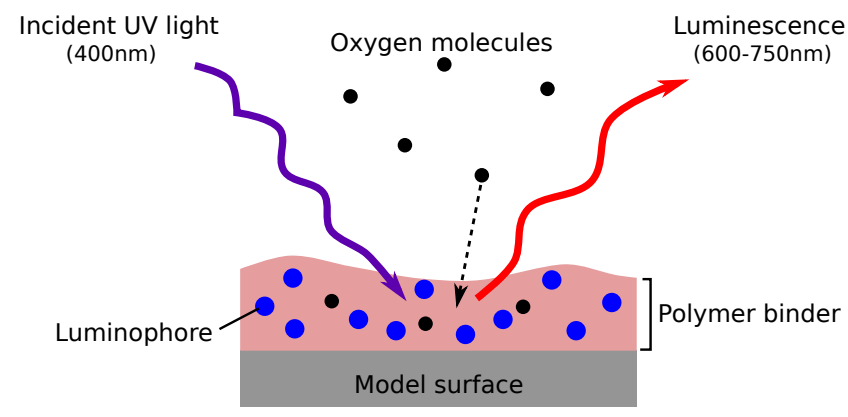

(b)

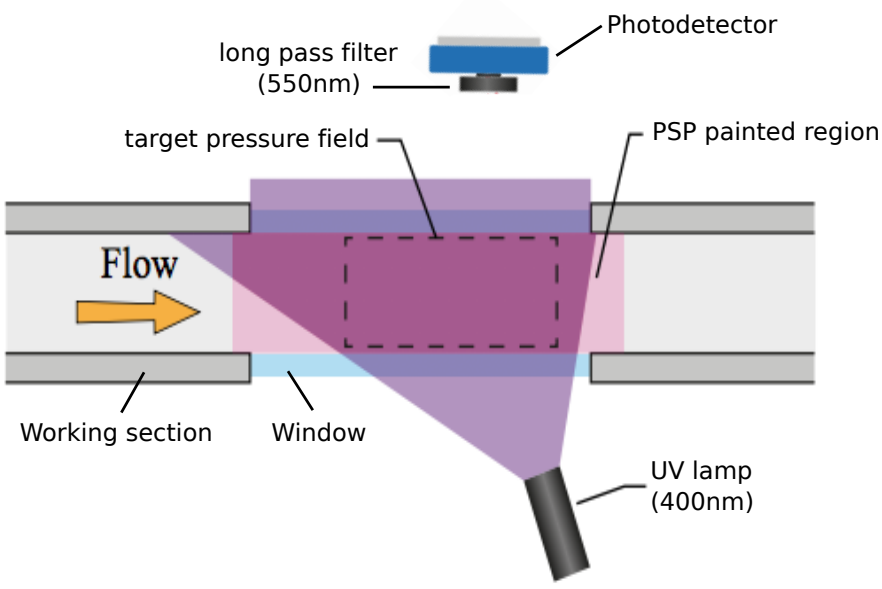

Fig. 3.11 Pressure sensitive paint technique: (a) underlying physics; $(b)$ experimental set-up, adapted from [34].

valid for a sufficiently thick painting. To achieve this, UniCoat PSP, supplied by Innovative Scientific Solution Incorporated (ISSI), is applied on the targeted wall in typically 5 layers. An ISSI LM2x-400 lamp serves to provide excitation light at 400nm, and luminescence is recorded on a Nikon D7100 from the other side of the wind tunnel, as shown in figure 3.11b. Ahead of the lens a long-pass filter of 550nm is used to block any incident UV light. A low aperture of $f / 8$ is used with a typical exposure time of $1 \mathrm{~s}$. This depth of focus enables the flow field all across the tunnel span to be clearly resolved.

An example PSP image for the tunnel floor is presented in figure 3.12. Here the light emission from the paint is predominantly red, and only the red channel in the RGB colour space is used for the reference image with no air flow (figure 3.12a) and the 'wind-on' image with tunnel started (figure 3.12b). By discarding data from the other channels, the image fidelity is improved. Following the Stern-Volmer equation, these two images are divided to obtain the relative intensity map (figure 3.12c). The calibration procedure is then conducted against the four pressure taps covering a large range of the pressure jump across 


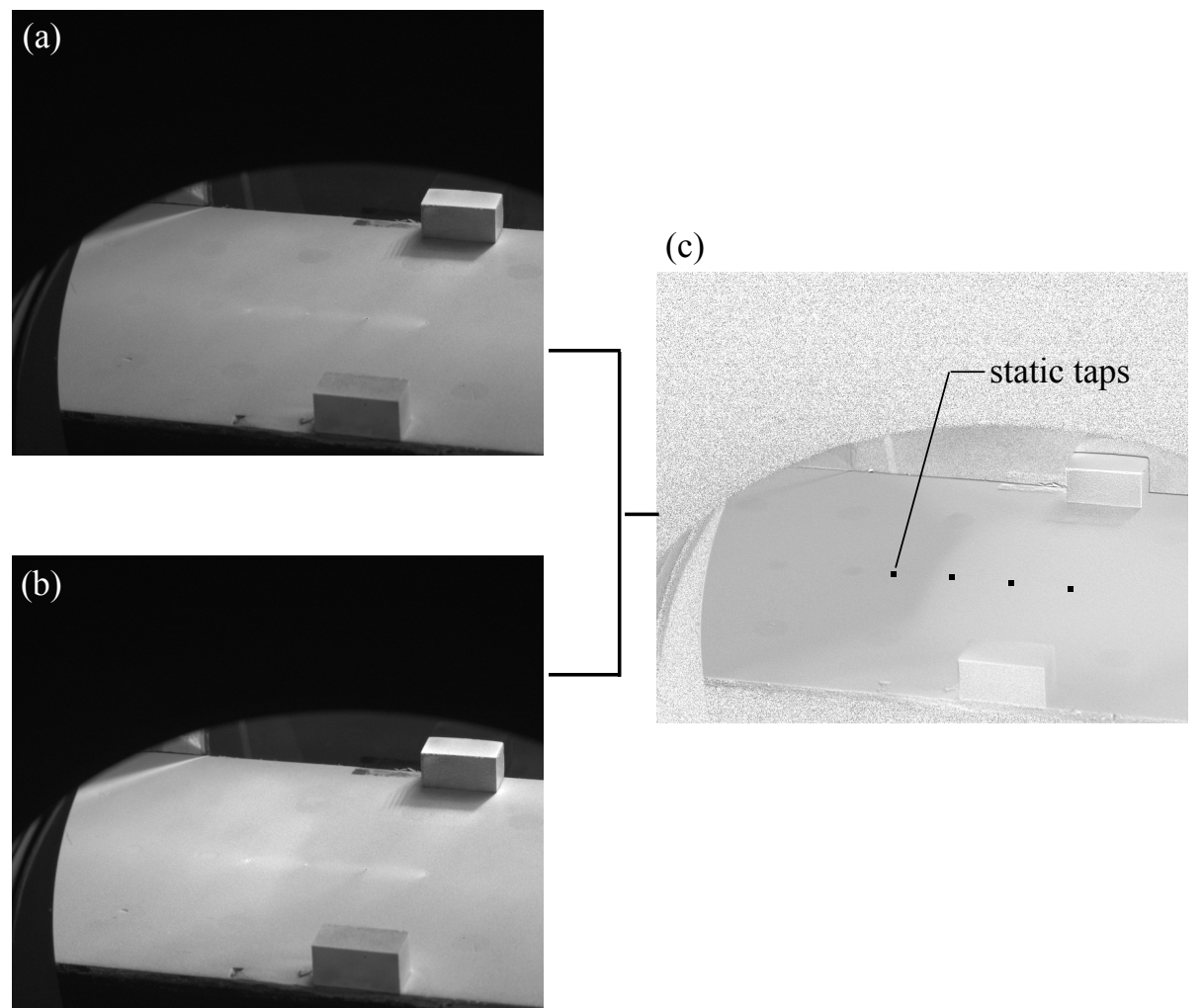

Fig. 3.12 PSP processing procedure: $(a)$ 'wind-off' image, $I_{r e f}$; $(b)$ 'wind-on' image, $I$; $(c)$ ratio of the two, $I_{r e f} / I$, tap locations shown by the squares.

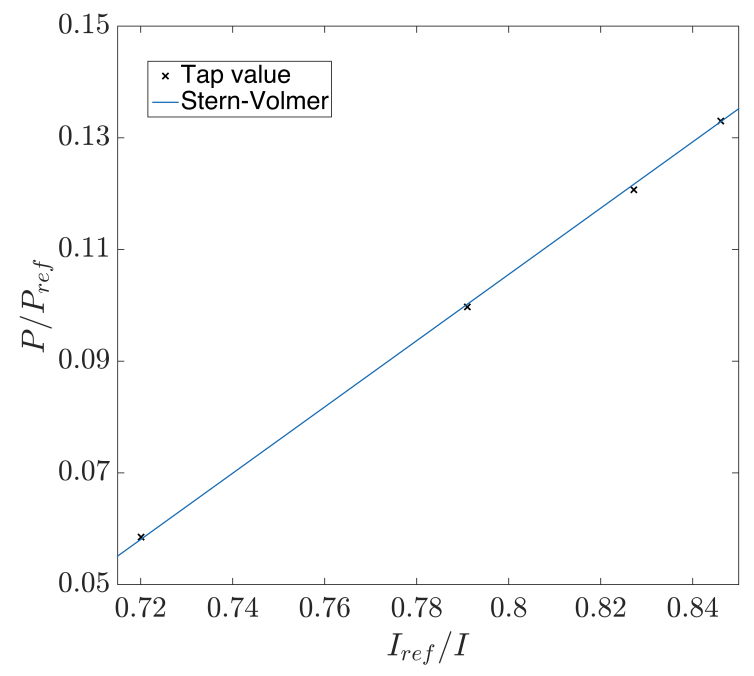

(a)

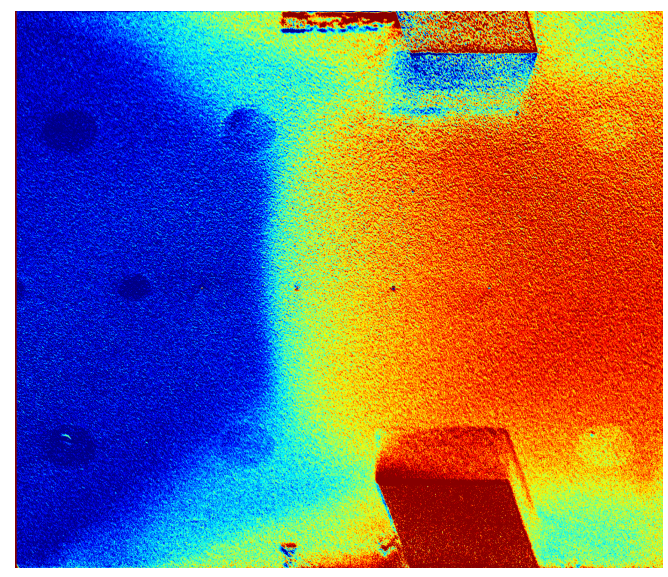

(b)

Fig. 3.13 PSP calibration: (a) calibration curve relating luminescence intensity and pressure; (b) map of the global pressure field. 
the oblique interaction. A typical calibration profile is shown in figure 3.13a, from which the light intensity is correlated with the pressures recorded by the NetScanner. The perspective view of the test section (a flat plate in this study) is corrected by a simple perspective transformation, with the resulting pressure map provided in figure 3.13b.

Static wall pressure distributions along the tunnel floor centreline are also obtained from the PSP images. Noise is reduced by averaging 15 spanwise pixels either side of the centreline (corresponding to a $1.5 \mathrm{~mm}$ span on the tunnel floor). Averaging over 10 pixels $(0.4 \mathrm{~mm})$ in the streamwise direction allows a further reduction in noise, without a significant deterioration of spatial resolution. As plotted in figure 3.14, this process gives a good agreement between the centreline pressure data extracted from the PSP map and the pressure taps.

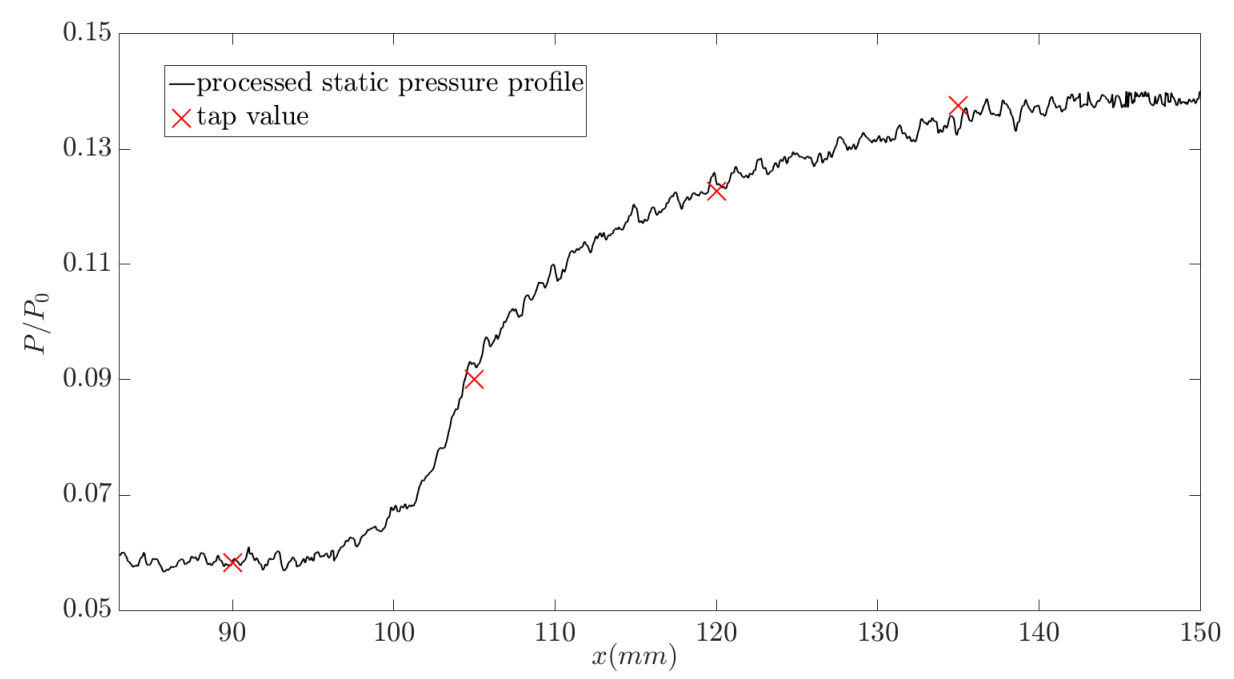

Fig. 3.14 Comparison of PSP data and pressure taps along the tunnel floor centreline.

The uncertainty for pressure sensitive paint measurements is dependent on a number of sources, with the key errors including thermal effects, the photodetector outputs and calibration accuracy.

Studies by Liu et al. [83] and Gregory et al. [64] have stated that temperature sensitivity is one of the most significant challenges affecting the accuracy of PSP data, through the coefficients in equations ( $3.4 \& 3.5$ ). In the Cambridge blow-down wind tunnel, the run times are short, typically 20s for a PSP test, and the surface temperature never reaches the thermal equilibrium state. Therefore, models with a significant thickness variation [34, 37] can give different responses to the same pressure, with an increased material thickness retarding the temperature reduction of the surface and hence suggesting a higher pressure. For the current investigation, pressure sensitive paint has been applied to the tunnel floor and wind tunnel sidewall, where there is no thickness change in the underlying surface material. The temperature influence is therefore significantly reduced. This is confirmed by comparing 
calibrated pressure fields with 'wind-off' images of supposedly different surface temperatures (i.e. take reference images both before and after a tunnel run), which show no noticeable difference. In addition, materials with different thermal properties also result in varied rates of temperature change, therefore affecting the static wall pressure readings. This can be seen in figure 3.13b, where the attachment screws on the tunnel floor and fillers in the corner regions are clearly visible due to the changed local temperatures.

Uncertainties in the photodetector outputs are due to noise and the limiting pressure resolution. For a given camera, when the full-well capacity of the sensor is achieved, one obtains the minimum pressure difference from a single frame of a PSP image [83]. For flows with low M numbers, the difference between the resting case and the 'wind-on' condition is relatively low, such that the photon shot noise can be a major concern. Sajben [108] proposed that the errors in pressure coefficients decreased monotonically with increasing free-stream M. An extended study by Mendoza [89] suggested that quantitative data of PSP system were limited to $M \geq 0.4$. In current study the incoming flow is at $M=2.5$, which is significantly higher than the minimum recommended $M$. McLachlan and Bell [87] have derived a first-order approximation for uncertainty due to photo shot noise:

$$
\Delta C_{p} \cong \frac{2}{B M^{2} \sqrt{m N}}
$$

where $\Delta C_{p}$ is the standard deviation given in terms of the pressure coefficient, $M$ is Mach number, $B$ is the slope of the Stern-Volmer calibration curve at atmospheric pressure, $N$ is the full-well capacity of the photodetector, and $\mathrm{m}$ is the number of images acquired and summed. It is calculated that with the capacity value for the Nikon D7100 being approximately 30000 electrons, the error related to photodetector sensitivity is only of the order of $10^{-2}$.

Calibration errors are introduced by uncertainties in determining the Stern-Volmer coefficients $A(T)$ and $B(T)$. The in-situ calibration adopted by current study fits the local luminescent intensity to the pressure tap data over the tested surface, which can significant reduce the bias error and achieve a better agreement with the pressure tap readings [83]. An example of such a calibration profile is depicted in figure 3.13a.

Combining all errors discussed above, the overall uncertainty of PSP results can be estimated by the deviation between the pressures extracted from the paint and the static tap measurements, as shown by the curve obtained by Nikon D7100 in figure 3.14. By examining all the curves acquired in this experimental investigation, an error bound of $\pm 3 \%$ can be placed on the calibrated PSP data (excludes measurements in regions where the thermal properties of the wall change). 


\subsection{Laser Doppler anemometry}

\subsubsection{General theory}

The principal velocity measurement technique used in the current study is Laser Doppler anemometry (LDA). For a detailed explanation the work of Durst et al. [50] is recommended; here only a brief description of the theory is presented.

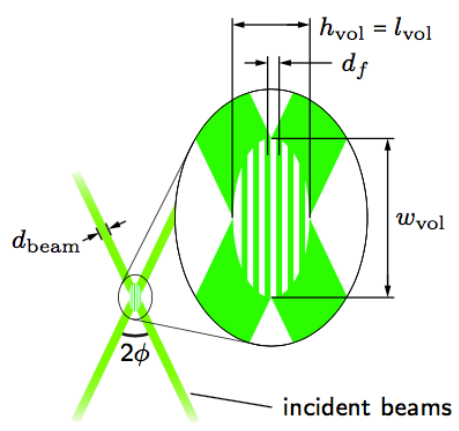

(a)

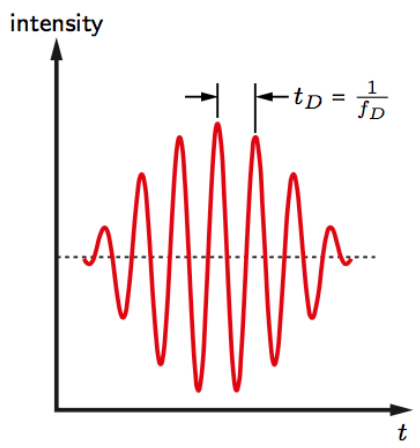

(b)

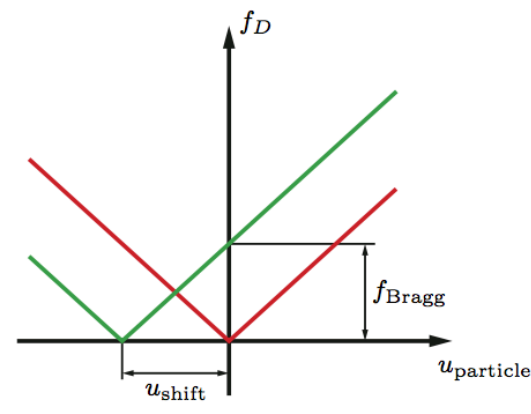

(c)

Fig. 3.15 Laser Doppler anemometry technique: (a) schematic of interference pattern in beam overlap volume; $(b)$ scattered light intensity variation due to a particle passing through the measurement volume, known as Doppler burst signal; $(c)$ relationship between velocity and Doppler frequency. [37]

When a pair of coherent laser beams intersect at a specific point in the flow, an interference pattern is produced, setting up a measurement volume with an ellipsoid shape. As depicted schematically in figure $3.15 \mathrm{a}$, the crossover region is characterised by variation in light intensity, with the spatial period of the fringes governed by the laser wave length $\lambda$ and the half-angle between the beam pairs $\phi$ :

$$
d_{f}=\frac{\lambda}{2 \sin \phi}
$$

The illuminating intensity distribution across the measured region has a Gaussian pattern, and the boundaries have been defined by Brayton [21] as where the intensity amplitude falls to $e^{-2}$ of the maximum value.

As a small particle moving with the flow traverses through this measurement volume, it scatters light from the bright fringes with a light intensity that oscillates in time. Such a signal is shown in figure 3.15b and is known as a Doppler burst. With the Doppler frequency $f_{D}$ recorded by the receiving photodetector, the particle velocity can be obtained by: 


$$
u=d_{f} f_{D}=\frac{\lambda}{2 \sin \phi} f_{D}
$$

The system described above however cannot differentiate between the forwards and backwards motion of a particle. This can be problematic when measuring through separated flow regions and determining the sign of the wall-normal velocity component. A Bragg cell is hence included in the beam manipulator, which imposes a frequency shift of $f_{\text {Bragg }}$ on one of the coherent laser beams, as shown in figure 3.15c. This makes the fringes travel upstream at a constant speed $u_{\text {shift }}$. The velocity measured by the Doppler burst is therefore biased by this shifted frequency, allowing negative velocities lower than the fringe-propagation speed to be detected.

\subsubsection{Current set-up}

It has been illustrated how a pair of coherent beams allows the velocity for one component to be determined with no ambiguity in flow direction. In this study a two-component Diode Pumped Solid State Laser system supplied by TSI is used, as sketched in figure 3.16a. Here two sets of laser beams, with wavelengths of $561 \mathrm{~nm}$ (yellow) and 532nm (green) respectively, operate at a power of $312 \mathrm{~mW}$, each measuring one velocity component. These beams are then steered through a lens with $L_{\text {focus }}=363 \mathrm{~mm}$ to ensure that they all focus at the same point in the working section. The laser configuration is aligned so that the incident beams are intersected at an angle of $2 \phi=7.88^{\circ}$. The frequency shift used is $f_{\text {Bragg }}=40 \mathrm{MHz}$.

The size of the measurement volume is predominantly determined by the height $l_{v o l}$ and width $w_{v o l}$ of the beam overlap (seen in figure $3.15 \mathrm{a}$ ), which are given by:

$$
\begin{gathered}
l_{\text {vol }}=\frac{4 \lambda L_{\text {focus }}}{\pi \cos (\phi) d_{\text {beam }}} \\
w_{\text {vol }}=\frac{4 \lambda L_{\text {focus }}}{\pi \sin (\phi) d_{\text {beam }}}
\end{gathered}
$$

Here $d_{\text {beam }}$ refers to the laser beam diameter which is quoted by the manufacturer as $2.10 \mathrm{~mm}$. According to equation (3.8), the velocities allowed to be measured are governed by the fringe spacing $d_{f}$ and the range of $f_{D}$ detectable, the latter of which can be varied by applying different band-pass filters. Based on an estimation of the anticipated velocity levels, the filter 
(a)
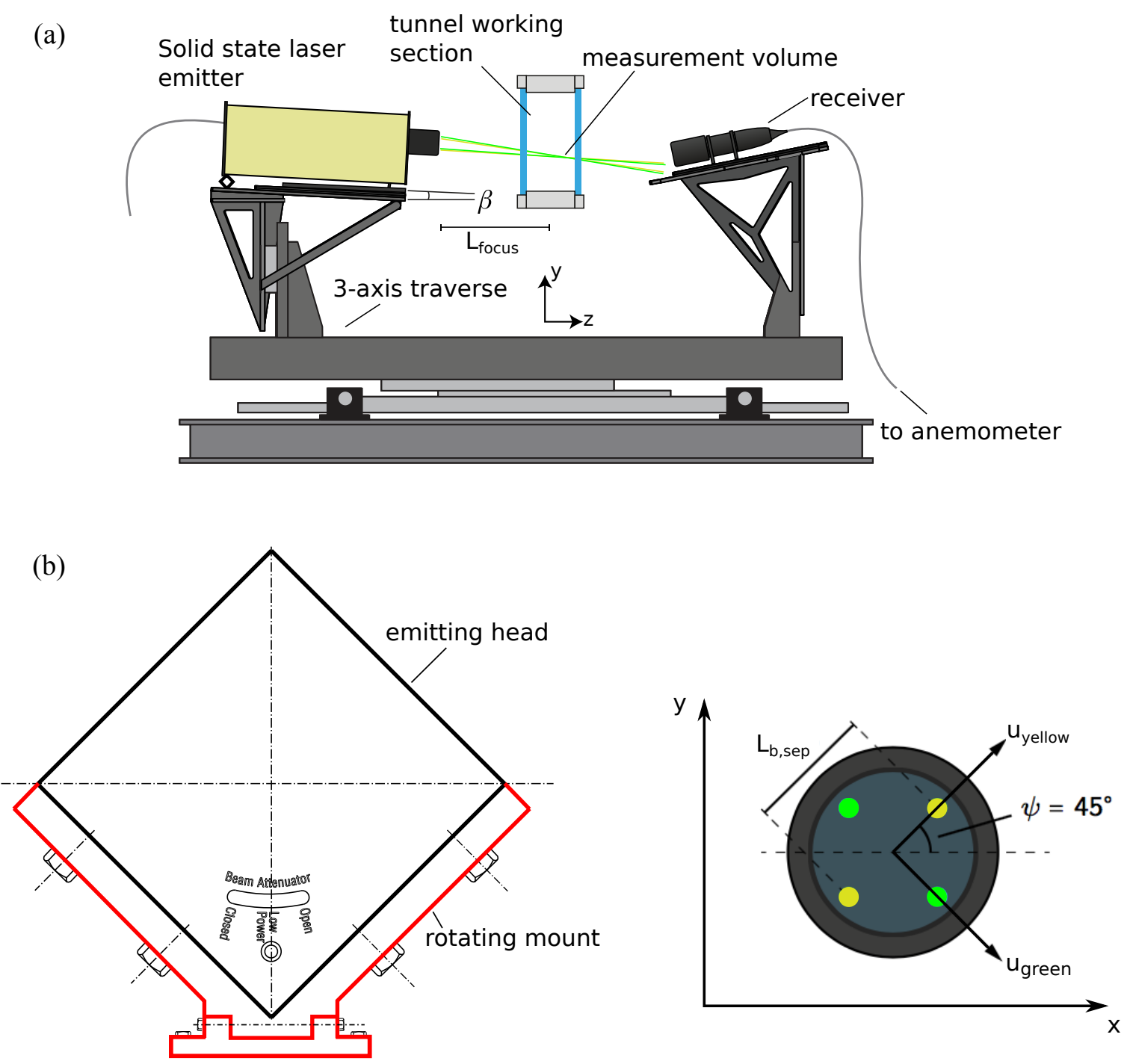

Fig. 3.16 Set-up of Laser Doppler anemometer: $(a)$ layout of the forward-mode equipment mounted on a 3-axis traverse, adapted from [35]; $(b)$ arrangement of the emitting head, shifted beams on the downstream side. 
is chosen based on the 'width' of data, since a narrower band makes for higher measurement resolution and lower noise. The detailed information of the current laser configuration is listed in table 3.2 .

Table 3.2 Details of the LDA configuration.

\begin{tabular}{lcc}
\hline Wavelength & $561 \mathrm{~nm}$ & $532 \mathrm{~nm}$ \\
\hline Focal length, $L_{\text {focus }}(\mathrm{mm})$ & \multicolumn{2}{c}{363} \\
Beam half-angle, $\phi$ & \multicolumn{2}{c}{$3.94^{\circ}$} \\
Beam separation length, $L_{b, \text { sep }}(\mathrm{mm})$ & \multicolumn{2}{c}{50} \\
Fringe spacings, $d_{f}(\mu \mathrm{m})$ & 4.082 & 3.871 \\
Laser beam diameter, $d_{\text {beam }}(\mathrm{mm})$ & \multicolumn{2}{c}{2.10} \\
Overlap waist, $l_{\text {vol }}(\mu \mathrm{m})$ & 123.76 & 117.36 \\
Overlap width, $w_{\text {vol }}(\mathrm{mm})$ & 1.80 & 1.70 \\
Band-pass filter $(\mathrm{MHz})$ & $60-150$ & $20-175$ \\
Minimum velocity $(\mathrm{m} / \mathrm{s})$ & 77 & -82 \\
Maximum velocity $(\mathrm{m} / \mathrm{s})$ & 425 & 550 \\
\hline
\end{tabular}

In this investigation the velocity in the plate-normal direction $v$ is one order of magnitude smaller than the streamwise velocity $u$, resulting in a far lower signal-to-noise ratio if measured directly. The emitting head is therefore aligned $45^{\circ}$ to the free-stream direction via the use of a 'V-shape' rotating mount, depicted in figure 3.16b. This enables the velocity components measured by both channels to carry similar magnitudes and hence signal-to-noise ratios. In addition, the emitter is also inclined by $\beta=3.5^{\circ}$ to the horizontal, to prevent the lower beams from being cut off as the measurement volume approaches the tunnel floor $\left(\beta>\tan ^{-1}(\tan (\phi) / \sqrt{2})\right.$ from simple geometry). This however introduces an additional error as a small fraction of the spanwise velocity $w$ is measured, with detailed analysis provided later.

The Doppler burst signals are collected by a RV 70 receiver focused on the measurement volume with a $500 \mathrm{~mm}$ focal length lens. As can be seen in figure 3.16a, the receiving optics are placed on the opposite side of the flow to the emitter, which is known as operating in forward-scatter mode. Following Hassal [67], such a configuration is more suitable for high-speed flows and transient phenomena like shock waves, as a greater signal-to-noise ratio can be achieved. The receiving optics are initially positioned by eye through a viewing point on one side, getting the crossing volume approximately in focus. Optimised alignment is then achieved by introducing seeding with tunnel off, and conduct fine adjustments until the recorded data rate is maximised.

The light signals captured by the receiver are then converted into voltages by a PDM 1000-2 photo-detector/multiplier. This is fed to an FSA 4000-2 frequency/burst spectrum 
analyser, which monitors the signal quality and determines the valid burst via a set of criteria such as 'amplitude threshold' and 'transit time' (the actual time particles are in the measured region). The extracted Doppler frequency is then passed to TSI's FlowSizer software, where velocity data is exported and post-processed.

The emitter and receiver are mounted on a motorised three-axis traverse, with the gear resolution being $0.01 \mathrm{~mm}$ for $x \& z$ and $0.005 \mathrm{~mm}$ in $y$. Continuous traverses are conducted both in the streamwise and wall-normal directions, allowing varied speeds up to $8 \mathrm{~mm} / \mathrm{s}$. As the traverse moves through the run, it is digitally controlled to wait $30 \mathrm{~ms}$ for vibration decay before averaged velocities are taken over 200ms. Data is only recorded when traversing in one direction to avoid backlash from affecting the position accuracy.

Locating the measurement volume in the wind tunnel is done by optical alignment. The spanwise location (z-axis) is confirmed by passing the four beams through a $35 \mu \mathrm{m}$ diameter pin-hole, which is mounted in an alignment jig. When the doors are closed, this assembly fixes the pin-hole at the centreline of the working section. The laser beams are traversed to intersect somewhere close to the pin-hole. One of the typical misaligned light patterns in figure 3.17a can be observed on the screen. A systematic scanning procedure using demanded traverse increments then enables the focused laser point to transmit through the pin-hole, which is achieved when the interference fringe pattern created by laser refracting is seen.

The position in wall-normal direction is checked by locating the tunnel floor before each run. As the crossing volume is brought down towards the surface, a strong reflection from where the beam intersects the floor becomes visible in the viewfinder of the receiver. This

(a)

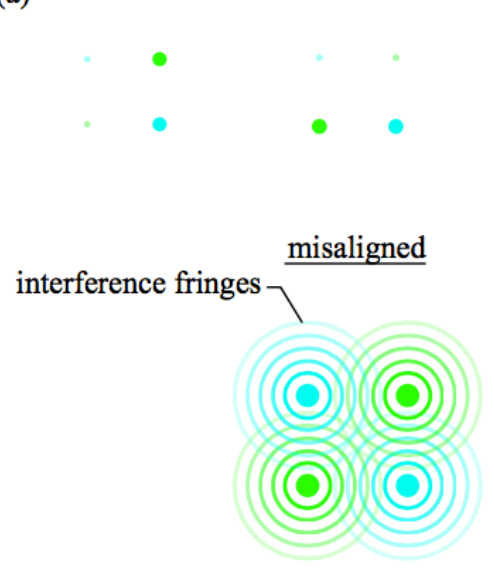

aligned (b)
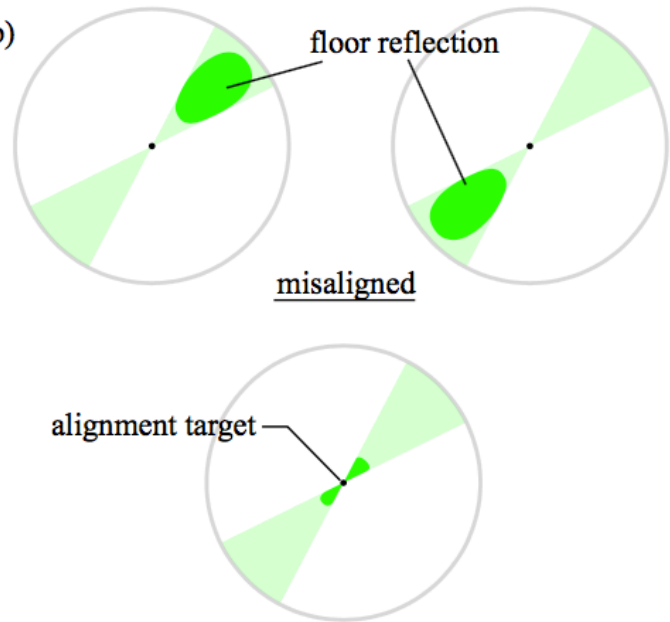

$\underline{\text { aligned }}$

Fig. 3.17 Example light patterns of laser alignment: (a) patterns through a $35 \mu \mathrm{m}$ pin-hole, interference fringes can be seen when aligned; $(b)$ floor-finding technique, even distribution around the alignment target indicates that the measurement volume is at the floor. [34] 
bright patch is then moved by traverse steps as fine as $0.005 \mathrm{~mm}$ in $\mathrm{y}$, until an even light distribution with equally strong reflection on both sides of the alignment target is observed, which indicates the measurement volume sits on the floor. This floor-finding technique is illustrated schematically in figure $3.17 \mathrm{~b}$, enabling an accuracy better than $\Delta y=0.01 \mathrm{~mm}$ (as two steps create an obvious deviation from the aligned pattern).

\subsubsection{Flow seeding}

The incoming flow is seeded with droplets of paraffin, generated by a pressurised seeder built in-house, as pictured in figure 3.18. High pressure air (typically $30 \sim 40$ psig above the setting chamber pressure) is fed to a double Laskin nozzle submerged into the paraffin reservoir, which atomises the oil into droplets and forces these particles into an impactor chamber. Here a series of perforated plates are applied to intercept and break up larger droplets, producing particles of relatively consistent size into the settling chamber. The seeding is introduced through a vertical rake in the symmetry plane of the settling chamber (shown in figure 3.1a). The rake spreads the droplets into a region $-15 \mathrm{~mm}<z<15 \mathrm{~mm}$ from the centre.

The size of seeding droplets is crucial for this experiment, as the investigated flow is characterized by large velocity gradients across oblique shock waves and expansion fans. Thomas et al. [125] have stated that in a fluid under the influence of sudden velocity changes, particles must be small to follow the flow with negligible lag; on the other hand, too small a diameter results in insufficient scatter-light intensity [91]. In the same facility, Colliss [34] estimated the mean diameter of the seeding particles as approximately $0.5 \mu \mathrm{m}$, by examining the particle lag through a normal shock and comparing it to theoretical models

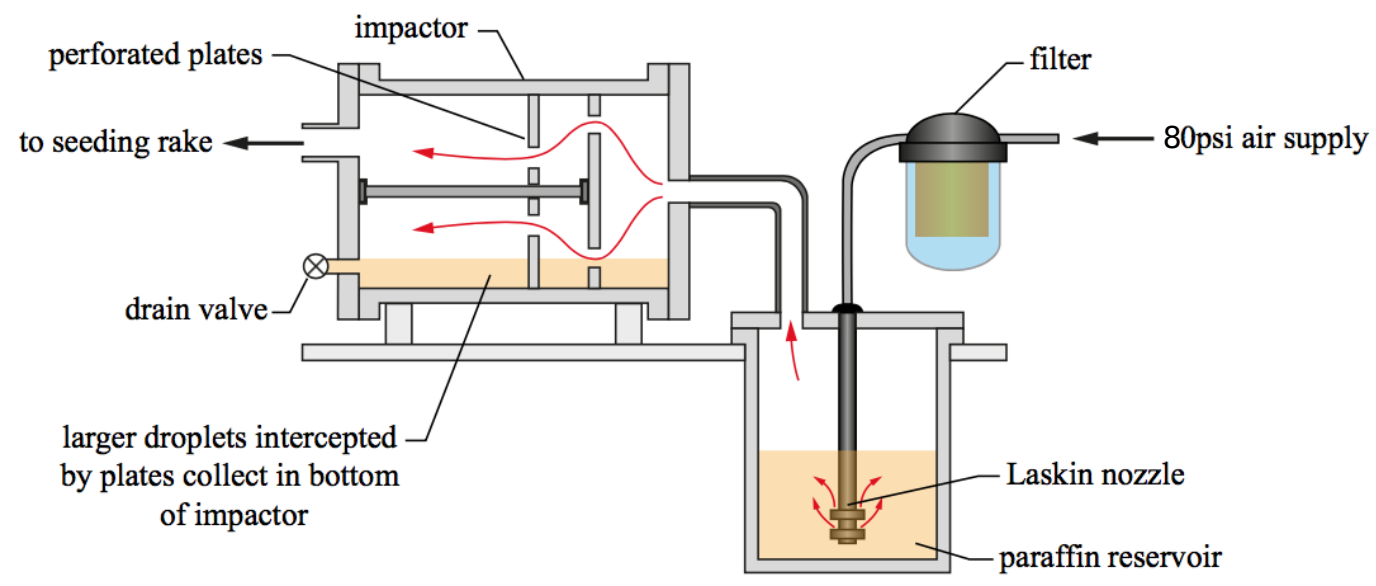

Fig. 3.18 Schematic of the seeding particle generator, with the path of paraffin droplets indicated by the red lines, adapted from [34]. 
of particle behaviour. Taking the inflow boundary layer thickness as $\delta=6 \mathrm{~mm}$, the Stokes number defined by:

$$
S_{k}=\frac{\rho_{p} d_{p}^{2} u_{\infty}}{18 \mu \delta_{o}}
$$

is valued at 0.06. Following McKeon et al. [86], $S_{k}<<1$ suggests the seeding can trace the flow very well.

The current velocity investigation focuses on the flow along the tunnel floor centreline, where the seeding density peaks. Figure 3.19a provides a typical seeding level across a continuous streamwise traverse in the free-stream, with the measurement volume moving through the oblique interaction at a rate of $4 \mathrm{~mm} / \mathrm{s}$. The valid burst rates are generally in the range of $20-35 \mathrm{kHz}$, ensuring that at least 4000 samples are collected per measurement location to calculate the time-averaged velocities in the outer flow. For boundary layer

(a)

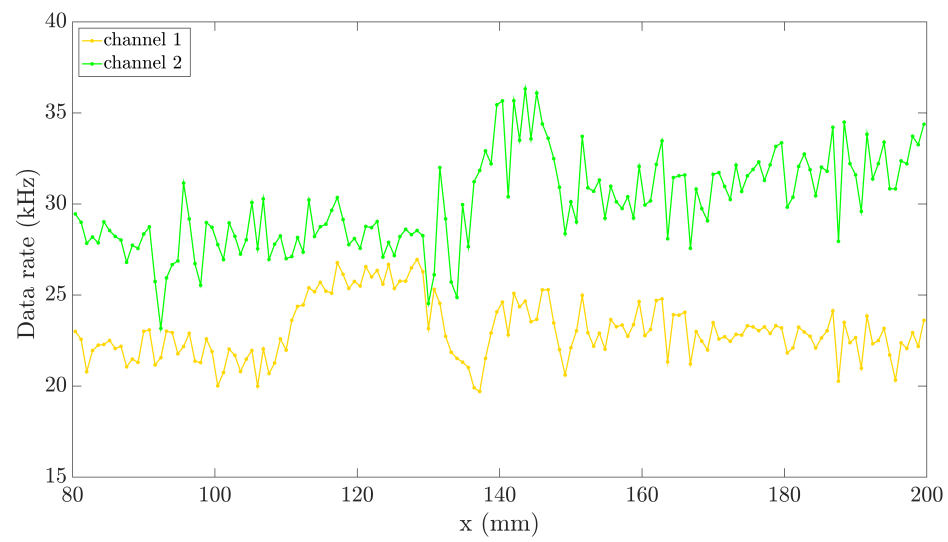

(b)
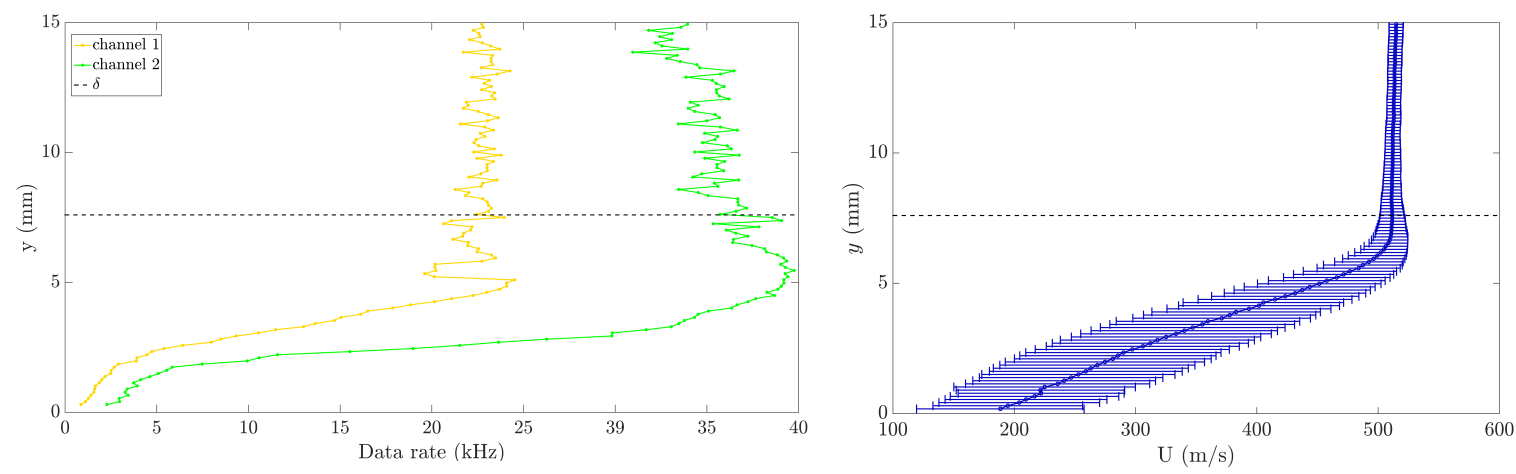

Fig. 3.19 Distribution of LDV data rate on the central symmetry plane: (a) streamwise data rates taken at $y=15 \mathrm{~mm} ;(b)$ boundary layer seeding rates and velocity data taken at $x=160 \mathrm{~mm}$. 
traverses, as shown in figure $3.19 \mathrm{~b}$, the data rate is considerably lower, and can fall to only a few $\mathrm{kHz}$ when approaching very close to the wall. For this reason, data measured within $0.2 \mathrm{~mm}$ of the tunnel are rejected. Furthermore, at each measurement station, samples outside $3 \sigma$ are rejected for the determination of mean velocities.

\subsubsection{Fitting boundary layer profiles}

In the current investigation boundary layer traverses have been conducted both at $x=$ $80 \mathrm{~mm}$ for the inflow, and also downstream of the oblique SBLI at $x=160 \mathrm{~mm}$. Accurate characterisation of the boundary-layer properties is essential to an understanding of the flow field, and this relies on incompressible integral parameters: displacement thickness $\delta_{i}^{*}$, momentum thickness $\theta_{i}$ and shape factor $H_{i}$. LDA measurements are located at discrete locations away from the wall, resulting in a discrete dataset. Integrating directly over the measured data points would underestimate the fullness of the boundary layer, due to the assumed straight lines in the gaps [131]. Also, as discussed in section 3.5.3, measurements any closer than $y=0.2 \mathrm{~mm}$ from the tunnel floor are considered invalid. In addition to the low seeding rates in the near-wall region, measurement volume (approximately $1.8 \mathrm{~mm}$ of width, seen in table 3.2) intersecting the wall at the bottom of a boundary layer is also a primary concern.

To address these problems, the measured points are filled to a suitable theoretical boundary-layer profile before calculating integrated parameters. A turbulent profile base on the model proposed by Sun and Childs [121] is commonly used at CUED [34, 37, 35]. This is based on the classical logarithmic law-of-the-wall (equation 3.12), together with the Coles wake function [33], which gives an expression of velocity in equation (3.13):

$$
\begin{gathered}
\frac{U}{u_{\tau}}=\frac{1}{\kappa} \log \left(\frac{y u_{\tau}}{v_{w}}\right)+B \\
\frac{U}{U_{e}}=1+\frac{1}{\kappa} \frac{u_{\tau}}{U_{e}} \log \left(\frac{y}{\delta}\right)-\frac{\Pi}{\kappa} \frac{u_{\tau}}{U_{e}}\left(1+\cos \left(\frac{\pi y}{\delta}\right)\right)
\end{gathered}
$$

The boundary layer profile following this definition however features a non-zero velocity gradient at the edge. Sun and Childs [121] therefore included a linear variation of shear stress across the boundary layer, as opposed to a constant value. Moreover, an adiabatic assumption is made due to the short run durations of the blow-down type tunnel and the 
stagnation temperature being close to the room temperature. The newly derived formulation for a turbulent boundary layer in an adiabatic flow is given as:

$$
\begin{gathered}
\frac{U_{i}}{U_{e}}=1+\frac{1}{\kappa} \frac{u_{\tau}}{U_{e}}\left[\log \left(\frac{y}{\delta}\right)+2 \sqrt{\frac{1-y}{\delta}}-2 \log \left(1+\sqrt{\frac{1-y}{\delta}}\right)\right]-\frac{\Pi}{\kappa} \frac{u_{\tau}}{U_{e}}\left(1+\cos \left(\frac{\pi y}{\delta}\right)\right) \\
\text { where } \frac{\Pi}{\kappa}=\frac{1}{2}\left[\frac{U_{e}}{u_{\tau}}-\frac{1}{\kappa} \ln \left(\frac{\delta u_{\tau}}{v_{w}}\right)-b+\frac{0.614}{\kappa}\right]
\end{gathered}
$$

To be valid for high-speed flows, this incompressible boundary layer profile is modified by the van Driest [133] transformation to account for the effects of compressibility:

$$
U_{v D}=\frac{U_{e}}{\sqrt{\Sigma}} \sin \left(\sqrt{\Sigma} \frac{U_{i}}{U_{e}}\right) \quad \text { where } \quad \Sigma=\frac{0.5(\gamma-1) M_{e}^{2}}{1+0.5(\gamma-1) M_{e}^{2}}
$$

This analytical profile is then fitted to the discrete data points in the logarithmic and wake regions, through built-in functions of Matlab by varying $\delta, c_{f}$ and $U_{e}$. Since locations with lower seeding rates have greater uncertainty, measurements are weighed by the local particle counts.

The above however does not describe the velocity distribution for the viscous sub-layer $\left(U^{+}=y^{+}\right.$till $\left.y^{+}=5\right)$ or the buffer zone $\left(y^{+}<100\right)$. The method of Musker [96] is therefore coupled within the fitting scheme to capture these regions:

$$
\frac{d U_{i}^{+}}{d y^{+}}=\frac{\frac{y^{+2}}{\kappa}+\frac{1}{s}}{y^{+3}+\frac{y^{+2}}{\kappa}+\frac{1}{s}}
$$

Here a cubic law is adopted for the variation of the eddy kinematic viscosity, which is verified by experimental results [96]. By using the skin friction coefficient $c_{f}$ generated from the Sun $\&$ Childs fit, the Musker-model is added to complete the boundary layer profile.

Two examples of the analytical profiles generated by the combined near-wall Musker model and Sun \& Childs model are plotted in figure 3.20. Figure 3.20a presents the boundary layer upstream of the oblique interaction. It can be seen that the model provides an excellent fit to the measured data, and that the profile closely resembles a naturally-grown zeropressure-gradient turbulent boundary layer in equilibrium. The profile of figure $3.20 \mathrm{~b}$ is taken downstream of the SBLI. Here the effect of the shock waves are shown clearly by the 
wake function extending almost all the way down to the blending region, and the skin friction coefficient is considerably lower than for the inflow. For all the boundary layers examined in this thesis, even in regions of adverse pressure gradient, a good fit is in general obtained, with $R^{2}$ coefficients greater than 0.995 . Therefore the shortcomings of integrating directly from the LDA data are overcome.

(a)

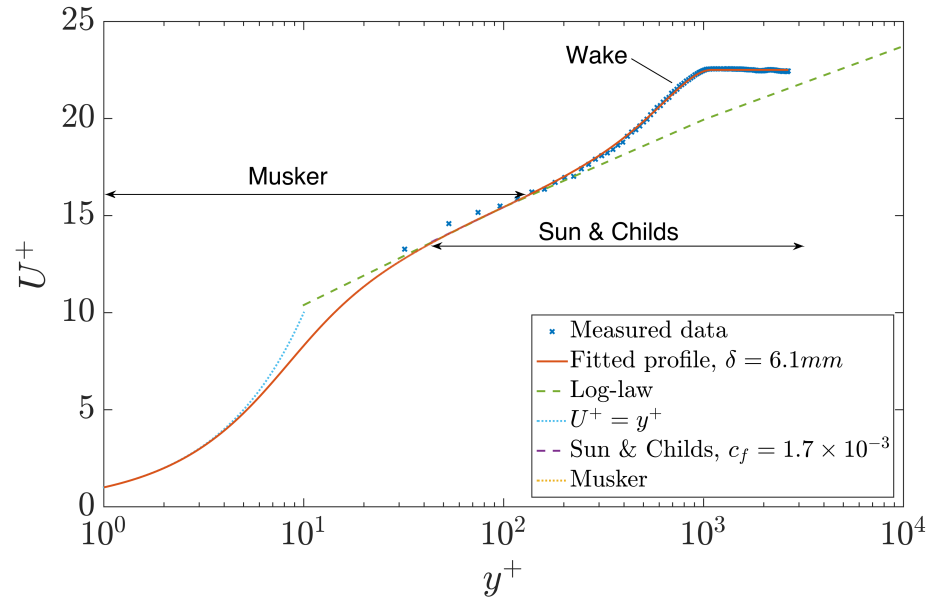

(b)

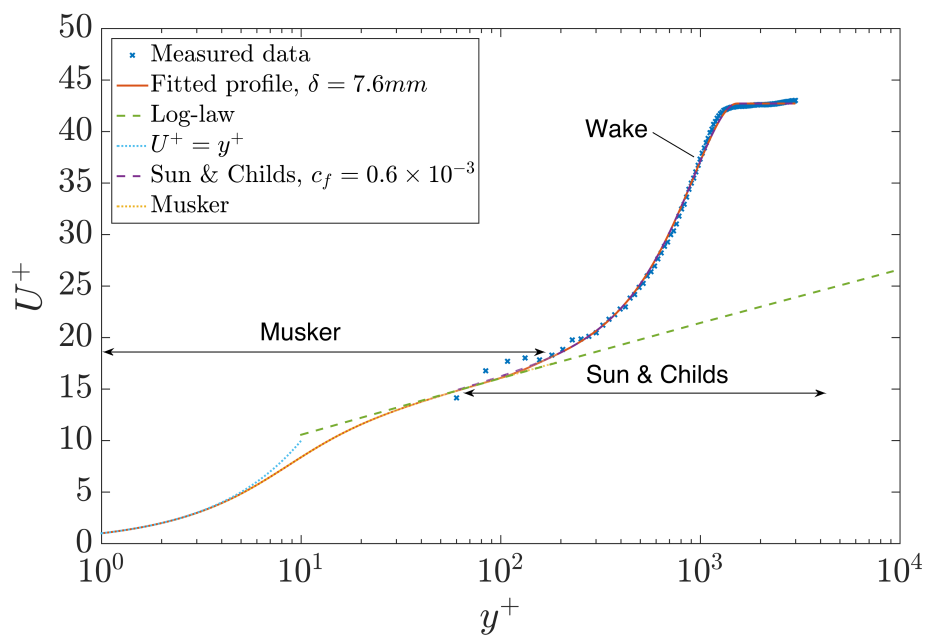

Fig. 3.20 Examples of analytical profiles fitted to discrete data in logarithmic wall units: $(a)$ upstream of oblique SBLI, at $x=80 \mathrm{~mm} ;(b)$ downstream of the interaction, at $x=160 \mathrm{~mm}$. 


\subsubsection{Uncertainty in LDA measurements}

The velocity reading of the LDA system, according to equation (3.8), is purely dependent on the measured frequency and the geometry of the laser beams. The capability of the anemometer itself is quoted by the manufacturer TSI to be $0.1 \%$ of the maximum velocity allowed by the band-pass filter [77]. In current investigation (refer to table 3.2), this gives an error of $\pm 0.55 \mathrm{~m} / \mathrm{s}$.

A far more significant error is related to the orientation of the emitting probe, including a rotation of $\Psi=45^{\circ}$ to the freestream direction and the pitch angle of emitter head at $\beta=3.5^{\circ}$. Such arrangements relate the measured velocities to the true velocities $(u, v, w)$ by:

$$
\begin{aligned}
& u_{\text {yellow }}=u \cos (\Psi)+v \sin (\Psi) \cos (\beta)+w \sin (\Psi) \sin (\beta) \\
& u_{\text {green }}=u \sin (\Psi)-v \cos (\Psi) \cos (\beta)-w \cos (\Psi) \sin (\beta)
\end{aligned}
$$

The head-angle rotation can be aligned to an accuracy of $\pm 0.25^{\circ}$ (via the rotating mount depicted in figure 3.16). The errors caused by the deviation $\delta \Psi$ from the true value can be estimated by: $|\delta u / u|=\delta \Psi v / u,|\delta v / v|=\delta \Psi u / v$. With the streamwise velocity being an order of magnitude larger than the wall-normal velocity, the resulting uncertainty is within $0.05 \%$ on $u$ and $5 \%$ on $v$.

The error introduced by the tilt of the emitting optics can be given by the velocities $(\tilde{u}, \tilde{v})$ transformed by the rotation matrix :

$$
\begin{gathered}
\tilde{u}=u \\
\tilde{v}=v \cos (\beta)+w \sin (\beta)
\end{gathered}
$$

It can be seen that while the stream-wise velocity is not influenced by this pitch angle, $\tilde{v}$ picks up a component of the span-wise velocity w. For the inclination of $\beta=3.5^{\circ}$ used here, $\tilde{v}$ measures $99.8 \%$ of the floor-normal velocity and $6 \%$ of the component in span-wise direction. Since velocity measurements are taken outside the separated regions, $v / w \sim 10$ can be expected throughout and the error in $\mathrm{v}$ is calculated at worst to be $0.5 \%$. 
Another source of error is velocity bias. Such biasing occurs because for higher velocities, a larger volume of fluid passes through the measurement volume, and hence a larger number of scattering particles is detected than for lower velocities. Consequently, a point measurement of a fluctuating velocity will be biased toward the faster end of the velocity range. According to the study of McLaughlin and Tiederman [88], this error is found to be $10 \%$ of the turbulent intensity. In the free-stream, $U^{\prime} / U_{e}$ presents an average of 0.02 , and so an uncertainty of approximately $0.2 \%$ for much of the flow. For the boundary layer traverses, the fluctuation intensity increases up to 0.4 , giving a $4 \%$ error due to velocity bias for measurements approaching the tunnel floor.

Finally, the errors of incompressible integral parameters calculated from the analytical profile are discussed; for more details, refer to Titchener et al. [131]. Discretization of the velocity profile is a primary error source. Figure 3.21 a shows the variation of the error in $\delta_{i}^{*}, \theta_{i}, H_{i}$ versus the uniform measurement resolution $\mathrm{N}$. It can be seen that as the number of measured points decreases, $\delta_{i}^{*}$ is over-predicted, $\theta_{i}$ is underestimated, and $H_{i}$ tends to be highly overestimated, thus being the parameter with the largest error. Figure $3.21 \mathrm{~b}$ plots the minimum discrete points required to give a $5 \%$ error, $N_{5} \%$, on all the parameters against the shape factor, from which a clear inverse relationship is presented. In the current investigation the data points collected inside the boundary layer range from 50 up to 80 , which gives an estimated error $<3 \%$ for the thinner inflow boundary layers and $<0.5 \%$ for the boundary layers with larger $H_{i}$ downstream of the oblique interaction.

Misalignment in the tunnel floor location are also found to impose a significant error in the integral parameters. Titchener et al. [131] have shown that a small wall location offset by $\Delta y / \delta=0.01$ can result in an error up to $6 \%$, with $\delta_{i}^{*}$ being most susceptible. For this thesis, the floor is aligned to $\Delta y / \delta=0.0017$ for the worst case (as the thinnest boundary layer is $\delta=6 \mathrm{~mm}$ ), which leads to an error in the integral parameters of less than $1 \%$ according to figure 3.21c.

Overall, the calculated incompressible integral parameter can be determined to an accuracy better than $1 \%$ for the downstream boundary layers; while the uncertainty increases slightly for the inflow boundary layers to be $\leq 4 \%$. 
(a)

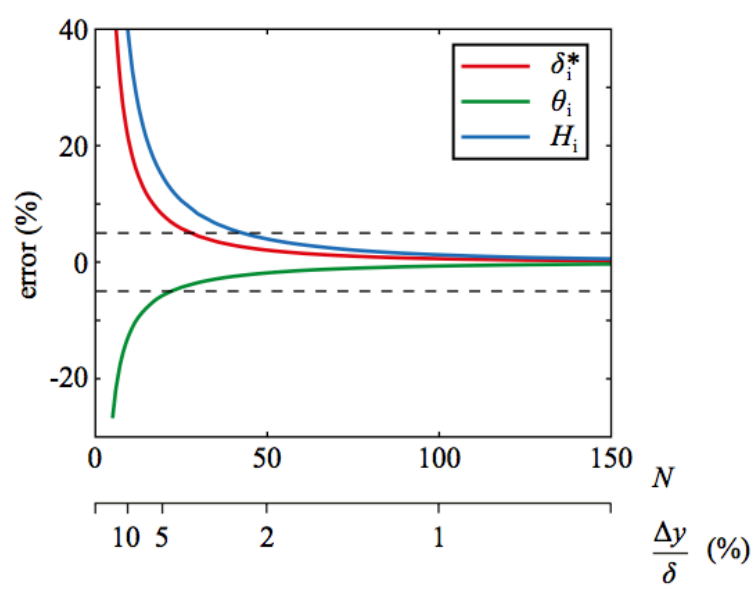

(b)

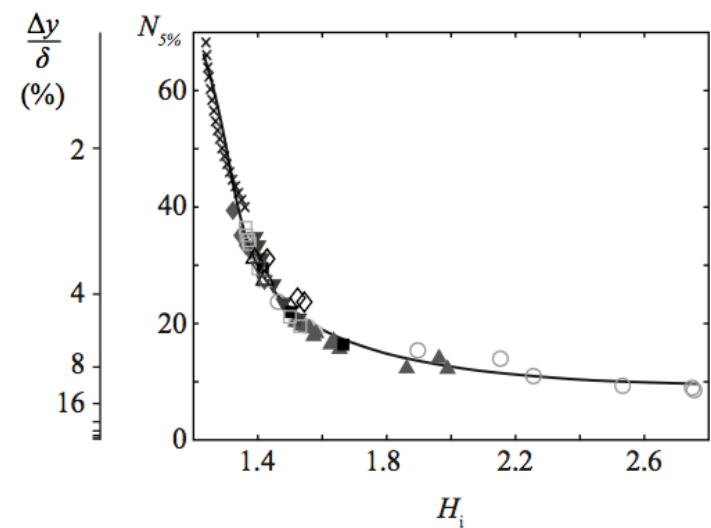

(c)

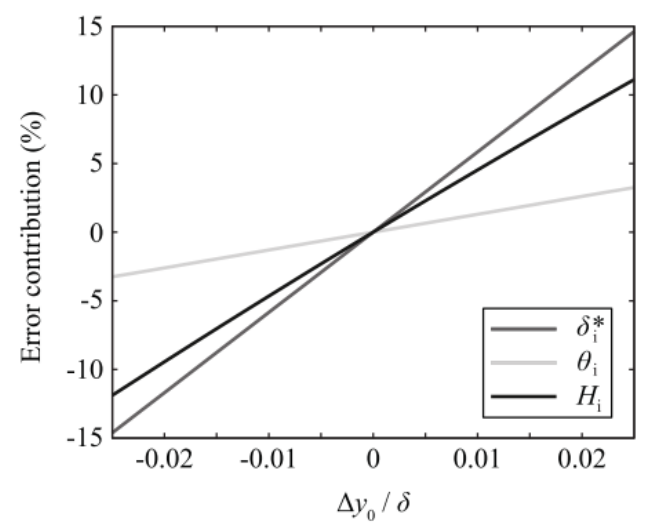

Fig. 3.21 Errors in calculated integral boundary layer parameters due to discretization: $(a)$ variation of errors against measurement resolution; $(b)$ critical measurement resolution required to give better than $5 \%$ error in all integral parameters; $(c)$ error caused by misalignment of the floor, from Titchener et al. [131]. 


\subsection{Experimental errors}

For each of the experimental techniques, various sources of errors have been discussed to determine the measurement accuracy. A summary of the final estimated uncertainties is provided in table 3.3 .

Table 3.3 Errors in the variables determined from experimental measurements.

\begin{tabular}{|c|c|c|}
\hline Quantity & Source & Error \\
\hline $\begin{array}{l}\text { Separation length } \\
\text { extracted from oil-flow, } L_{\text {sep }}\end{array}$ & $\begin{array}{l}\text { early indication of } \\
\text { separation location }\end{array}$ & $+5 \%$ \\
\hline \multicolumn{3}{|l|}{ Stagnation Pressure, $P_{0}$} \\
\hline & atmospheric pressure & $\pm 0.05 \%$ \\
\hline & pressure transducer & $\pm 0.05 \%$ \\
\hline & settling chamber velocity & $-0.01 \%$ \\
\hline \multicolumn{3}{|l|}{ Static Pressure, $P$} \\
\hline & atmospheric pressure & $\pm 0.05 \%$ \\
\hline & pressure transducer & $\pm 0.05 \%$ \\
\hline & static tap geometry & $\pm 0.5 \%$ \\
\hline & pressure sensitive paint & $\pm 2.5 \%$ \\
\hline \multicolumn{3}{|l|}{ Streamwise velocity, $u$} \\
\hline & Doppler frequency detection & $\pm 0.1 \%$ \\
\hline & emitting head rotation $\Psi$ & $\pm 0.05 \%$ \\
\hline & velocity bias in free-stream & $+0.2 \%$ \\
\hline & velocity bias near wall & $+2 \%$ \\
\hline \multicolumn{3}{|l|}{ Wall-normal velocity, $v$} \\
\hline & Doppler frequency detection & $\pm 0.1 \%$ \\
\hline & emitting head rotation $\Psi$ & $\pm 5 \%$ \\
\hline & emitting head inclination $\beta$ & $\pm 0.5 \%$ \\
\hline & velocity bias in free-stream & $+0.2 \%$ \\
\hline & velocity bias near wall & $+2 \%$ \\
\hline \multicolumn{3}{|l|}{$\delta_{i}^{*}, \theta_{i}, H_{i}$} \\
\hline & inflow boundary layer at $x=80 \mathrm{~mm}$ & $\pm 4 \%$ \\
\hline & discretisation & $\pm 3 \%$ \\
\hline & wall alignment & $\pm 1 \%$ \\
\hline & downstream boundary layer at $x=160 \mathrm{~mm}$ & $\pm 1 \%$ \\
\hline & discretisation & $\pm 0.5 \%$ \\
\hline & wall alignment & $\pm 0.5 \%$ \\
\hline
\end{tabular}




\section{Chapter 4}

\section{Experimental investigation}

In the present chapter the corner flows for an established reflecting shock interaction are manipulated to produce corner separations varying in relative size from moderate to significant. The aim is to determine how the relative magnitude of corner separation modifies the size and shape of 'quasi-two-dimensional' separation observed in the centre of a duct.

Experiments are undertaken at $M=2.5$ with a unit Reynolds number of $40 \times 10^{6} \mathrm{~m}^{-1}$. A flow deflection of $8^{\circ}$ is generated by a wedge mounted at the ceiling of the working section. Previous studies in the same wind tunnel have shown that such a wedge angle can result in an oblique interaction with substantial separations both in the corners and along the centreline. This is thought to be a good condition for the baseline flow, which is presented first. A number of modifications to the corner flow geometry will then be introduced. The chapter will conclude with an analysis of flow parameters relevant to shock-induced separations, with the overarching goal of determining a suitable indicator to describe the impact of corner effects for an oblique SBLI.

\subsection{Baseline flow}

Figure 4.1 shows a schlieren image of the unmodified baseline interaction. The standard pattern of a separation and reattachment shock bounding an expansion fan suggests the presence of a separated region. The oblique interaction domain spreads from $x=110 \mathrm{~mm}$ to $x=140 \mathrm{~mm}$, with the inviscid shock reflection located at $x=135 \mathrm{~mm}$. At the top of the image, a strong expansion fan emanates from the downstream wedge corner, impinging on the tunnel floor at approximately $x=200 \mathrm{~mm}$, roughly $10 \delta$ behind the inviscid shock reflection location.

The streamwise velocity variation measured along $y=15 \mathrm{~mm}$ on the central symmetry plane (marked by a white dot-dashed line in figure 4.1) is shown in figure 4.2. The flow is 


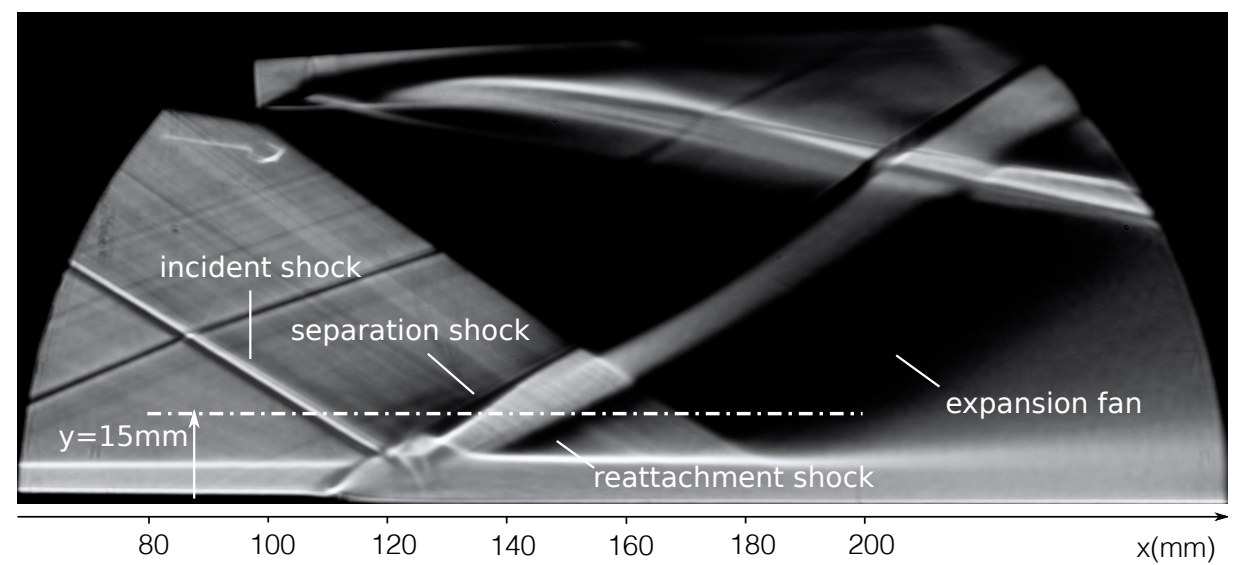

Fig. 4.1 Schlieren photograph for the baseline flow: $M=2.5$, wedge angle $=8^{\circ}$.

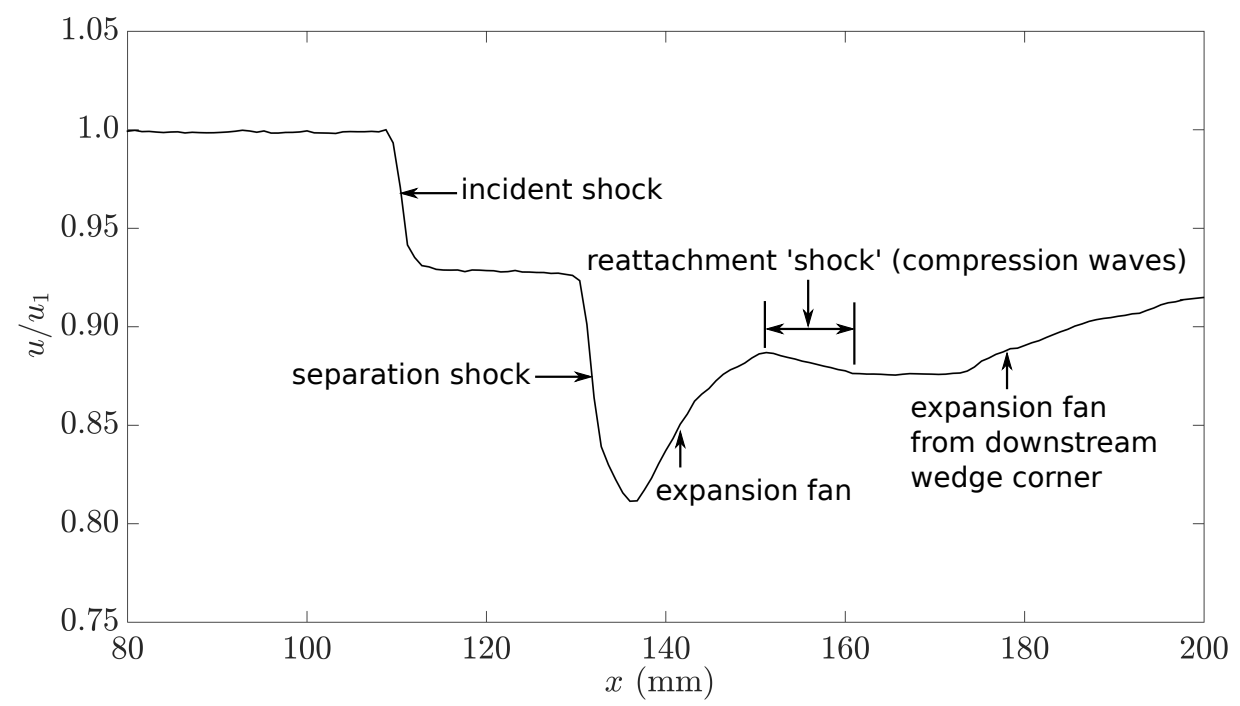

Fig. 4.2 Variation in streamwise velocity at $y=15 \mathrm{~mm}, z=0 \mathrm{~mm}$ for the baseline flow.

initially at the incoming free-stream velocity $\left(u_{1}\right)$, and then decelerates through the incident shock. The second deceleration step corresponds to the separation shock, which is followed by a re-acceleration region through the expansion fan. Deceleration at reattachment presents a more gentle trend than at separation, indicating that the reattachment shock is weak and quite smeared-out even in the outer inviscid flow. Approximately $15 \mathrm{~mm}$ downstream of the oblique SBLI, the flow is seen to be accelerated by the expansion waves originating from the downstream corner of the shock generator. The LDA measurements are in good agreement with the separated wave patterns depicted in the schlieren photograph.

Figure 4.3 presents the boundary layer profiles measured at two streamwise positions along the centreline. Here the inflow boundary layer obtained at $x=80 \mathrm{~mm}$, shown in fig- 


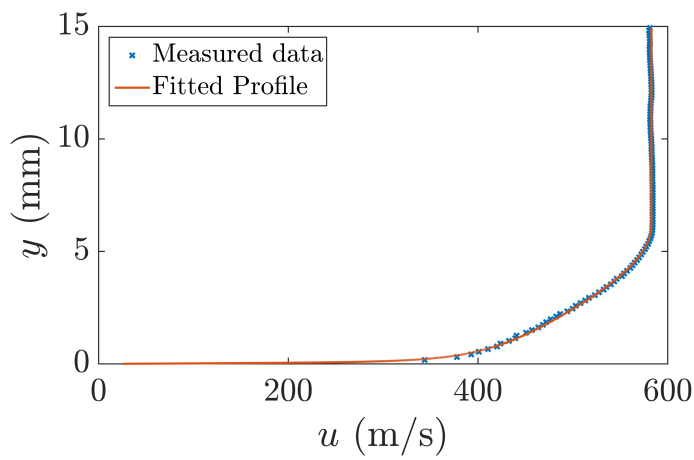

(a)

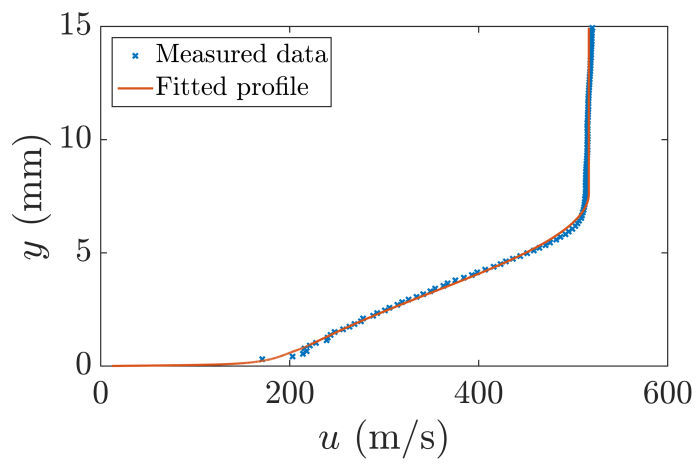

(c)

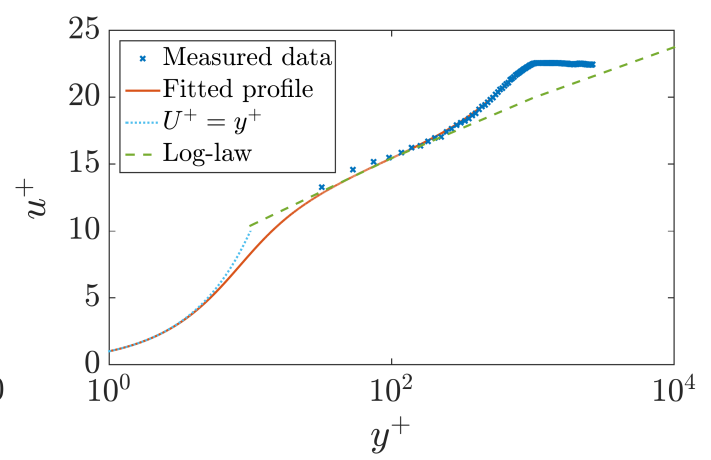

(b)

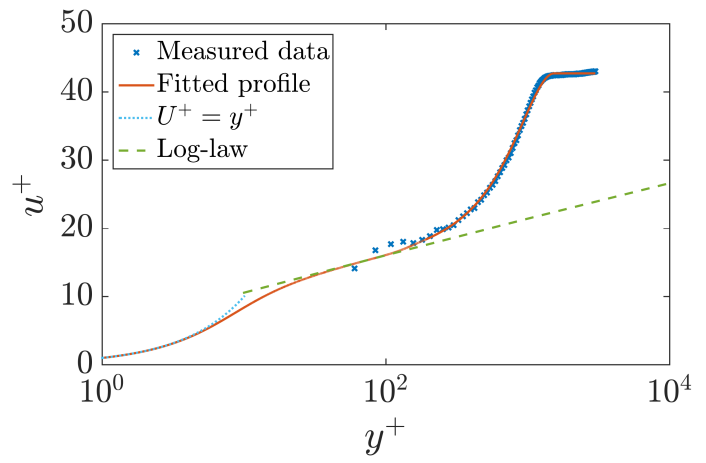

(d)

Fig. 4.3 Boundary-layer profiles measured with LDA along the tunnel floor centreline for the baseline case: $(a)$ inflow boundary layer profile at $x=80 \mathrm{~mm} ;(b)$ inflow boundary layer profile in law-of-the-wall coordinates; $(c)$ downstream boundary layer profile at $x=160 \mathrm{~mm}$; $(d)$ downstream boundary layer profile in law-of-the-wall coordinates.

ures $4.3 \mathrm{a}$ and $4.3 \mathrm{~b}$, is $6.1 \mathrm{~mm}$ thick, and the incompressible integral values are: displacement thickness $\delta^{*}=0.84 \mathrm{~mm}$, momentum thickness $\theta=0.61 \mathrm{~mm}$, and shape factor $H=1.37$. There is a well-defined log region and the agreement with the analytical profile is excellent throughout. The results demonstrate that the inflow boundary layer closely resembles a naturally-grown zero-pressure-gradient turbulent boundary layer in equilibrium. A rapid thickening of the boundary layer can be observed across the interaction in the schlieren image. The boundary layer measured at $x=160 \mathrm{~mm}$ (figures $4.3 \mathrm{c}-\mathrm{d}$ ), around $4 \delta$ downstream of the inviscid shock reflection, is $7.6 \mathrm{~mm}$ thick, with a displacement thickness of $2.14 \mathrm{~mm}$, momentum thickness of $1.17 \mathrm{~mm}$ and a shape factor of 1.83 . It can be seen that while increase in $\delta$ is relatively mild, $\delta^{*}$ has more than doubled after the SBLI.

Figure 4.4 shows the oil-flow visualisation on the tunnel floor. It can be seen that the locations of separation and reattachment are clearly identifiable for the centreline separation. In each corner region, the presence of reversed flow is suggested by the displacement of the incoming streamlines. The oil streaklines also allow the critical points to be identified, with 
the skin-friction lines whirling around beneath the focus of a separation while adopting the shape of a hyperbolic curve approaching the saddle point.

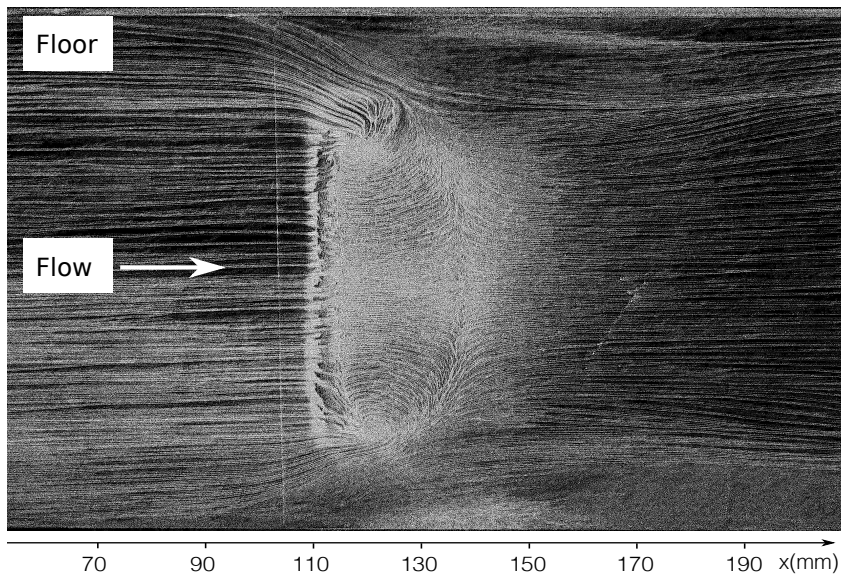

Fig. 4.4 Oil-flow visualisation on the tunnel floor for the baseline flow.

(a)

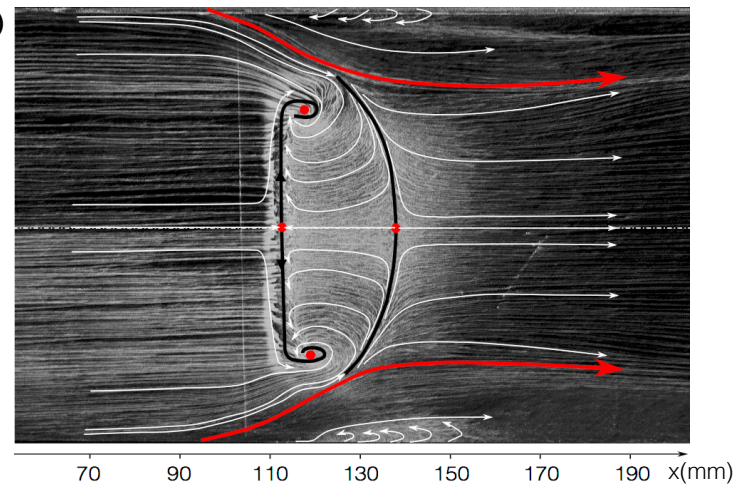

(b)

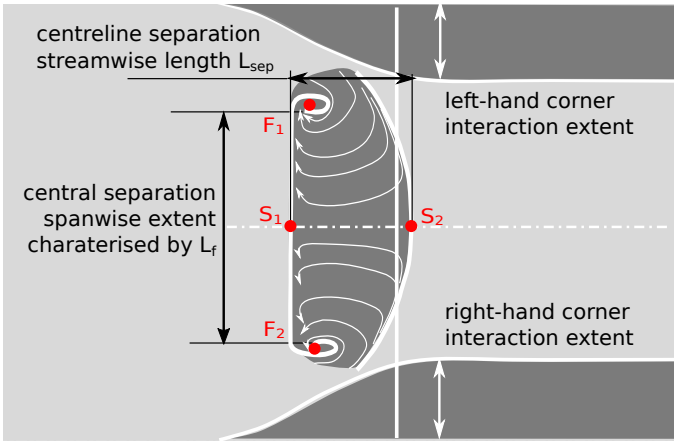

Fig. 4.5 Interpretation of flow topology on the tunnel floor for the baseline flow by $(a)$ surface oil-flow visualisation; $(b)$ schematic illustration of separation sizes extracted from the oil flow. Limiting streamlines and the footprint of the separation are suggested for clarity; the vertical line indicates the inviscid shock location.

Interpretation of the oil-flow image on the tunnel floor is provided in figure 4.5. As shown in figure $4.5 \mathrm{a}$, the corner separation regions are indicated with a red line, while the black lines mark the centreline separation and reattachment locations. Limiting streamlines are also suggested for clarity, with the surface flow topology presented and annotated schematically in figure 4.5b. Here the inviscid shock location, from an extrapolation of the incident shock seen in figure 4.1 , is marked by a vertical line. The skin friction lines suggest that the centreline separation topology falls into the 'owl-face of the first kind' category, with two saddle points (S) and a pair of foci (F) clearly identifiable. The sizeable separation region in the centre of the tunnel exhibits a relatively two-dimensional pattern for much of its spanwise 
extent. However towards the sidewalls the streamwise separation length reduces and the separated region is bounded by distinct foci on either side. On the centreline the separation starts at $x=112 \mathrm{~mm}$, measuring $25 \mathrm{~mm}$ in streamwise direction (termed $L_{s e p}$ ). The spanwise separation width $L_{f}$ is defined as the distance between the two foci and covers $62 \mathrm{~mm}(54.4 \%$ of working section width). Corner separations are seen to originate approximately $18 \mathrm{~mm}$ ahead of the centreline separation. The left-hand corner interaction (referring to the corner location relative to the incoming flow) 'footprint' is seen to extend over $21 \mathrm{~mm}$ from the side wall of the working section on the floor ( $18.4 \%$ of full span), while the right-hand corner interaction covers a $23 \mathrm{~mm}$ spanwise distance (20.2\% of full span). Although critical points are not clearly seen in the corners, the recirculating streamlines suggest the presence of a focus and the formation of a corner vortex on both sides. A narrow attached channel is observed between the central and corner separation.

Figure 4.6 presents the static wall pressure distribution for the tunnel floor. The simplified separation topology is superimposed on the PSP map to allow a comparison with the oil-flow visualisation. It can be seen that there is a uniform region of low pressure upstream of the oblique interaction. This is followed by a rapid pressure rise starting near the separation onset. This separation shock footprint presents little spanwise variation, except for the additional pressure smearing as the sidewalls are approached. Comparing the pressure distribution with the separation boundaries, it can be seen that the more upstream pressure rise near

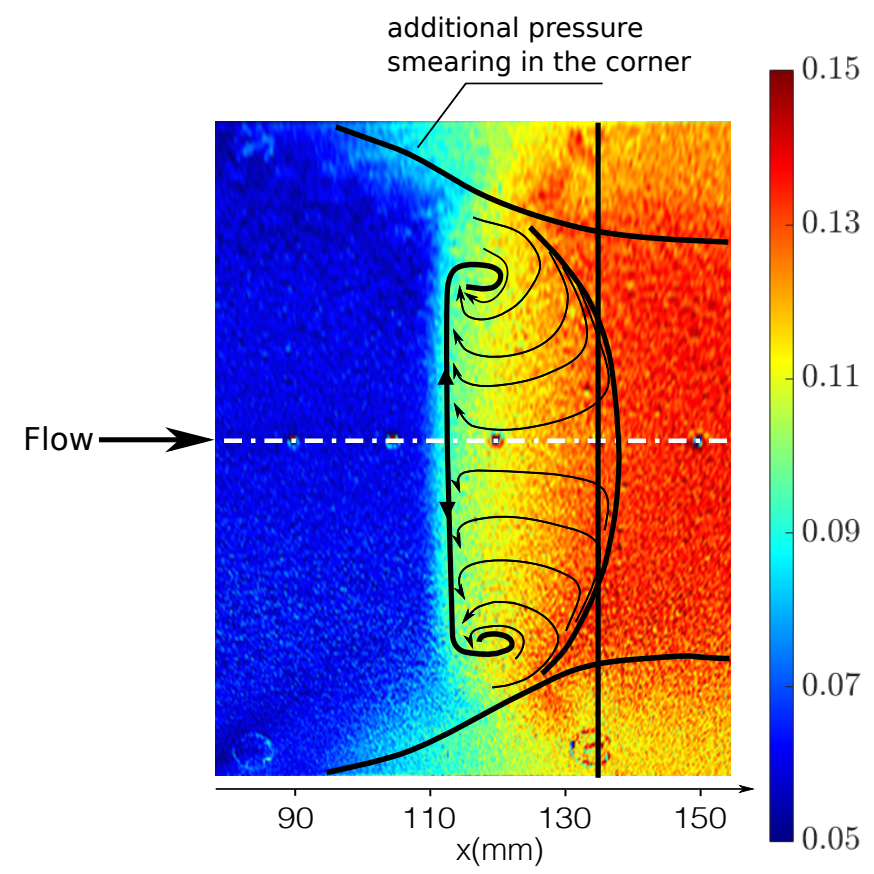

Fig. 4.6 $P / P_{0}$ on the tunnel floor for the baseline flow. 


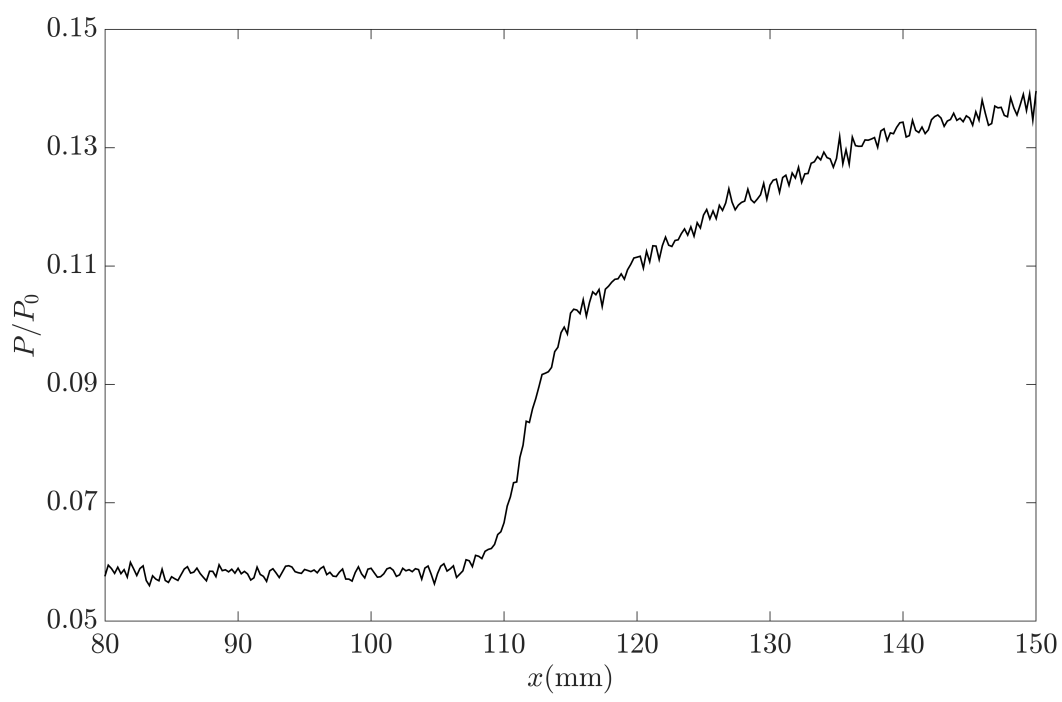

Fig. 4.7 Static wall pressure distribution along the tunnel floor centreline for the baseline flow.

the sidewalls corresponds well to the onset of corner separation. This smeared-out corner pressure distribution is therefore likely linked to the corner separation.

The static pressure distribution along the tunnel floor centreline is provided in figure 4.7. Although a plateau pressure corresponding to the separated region might be expected, it is shown that the plateau region is in fact smeared into a more gradual pressure rise. The significant upstream influence associated with reversed flow underneath the shock is also confirmed, with the pressure rise occurring at $x=108 \mathrm{~mm}, 27 \mathrm{~mm}$ ahead of the shock foot in the inviscid-flow model.

Figure 4.8 shows the oil-flow image of the corner region on the right sidewall. A defined focus can be observed at the edge of corner separation. The separation is seen to start at $x=87 \mathrm{~mm}$ and cover a maximum span of $18 \mathrm{~mm}$.

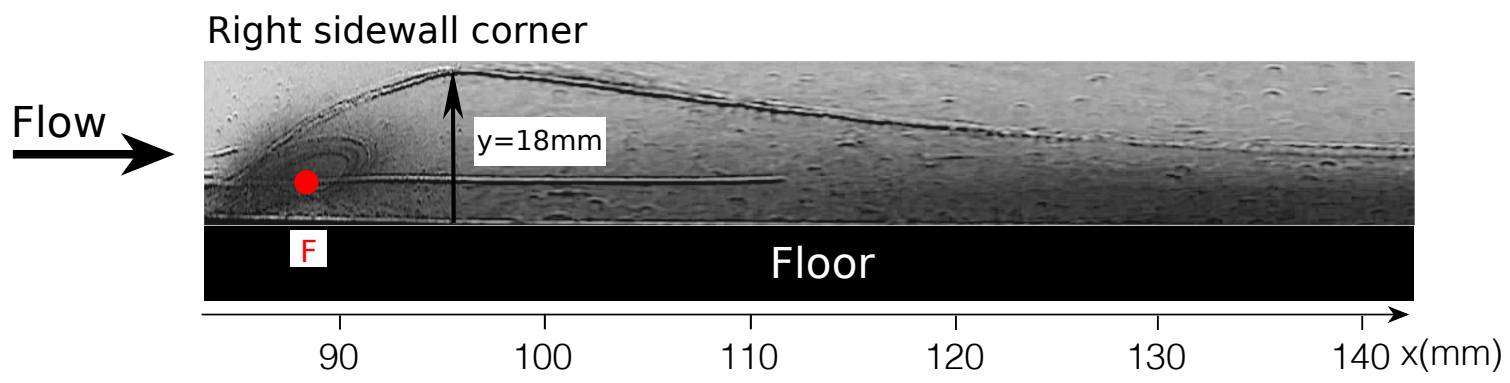

Fig. 4.8 Oil-flow visualisation in the right sidewall corner for the baseline flow. 


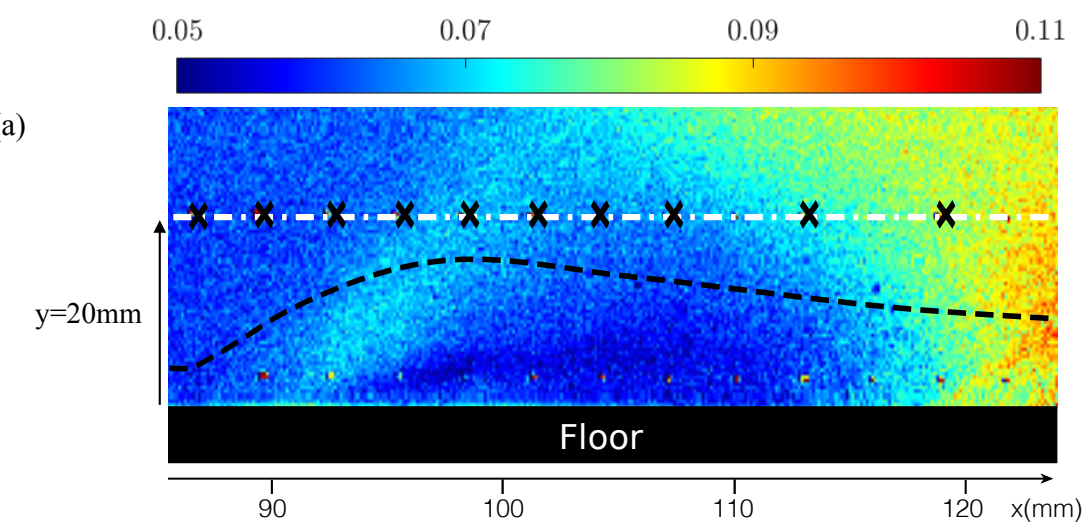

(b)

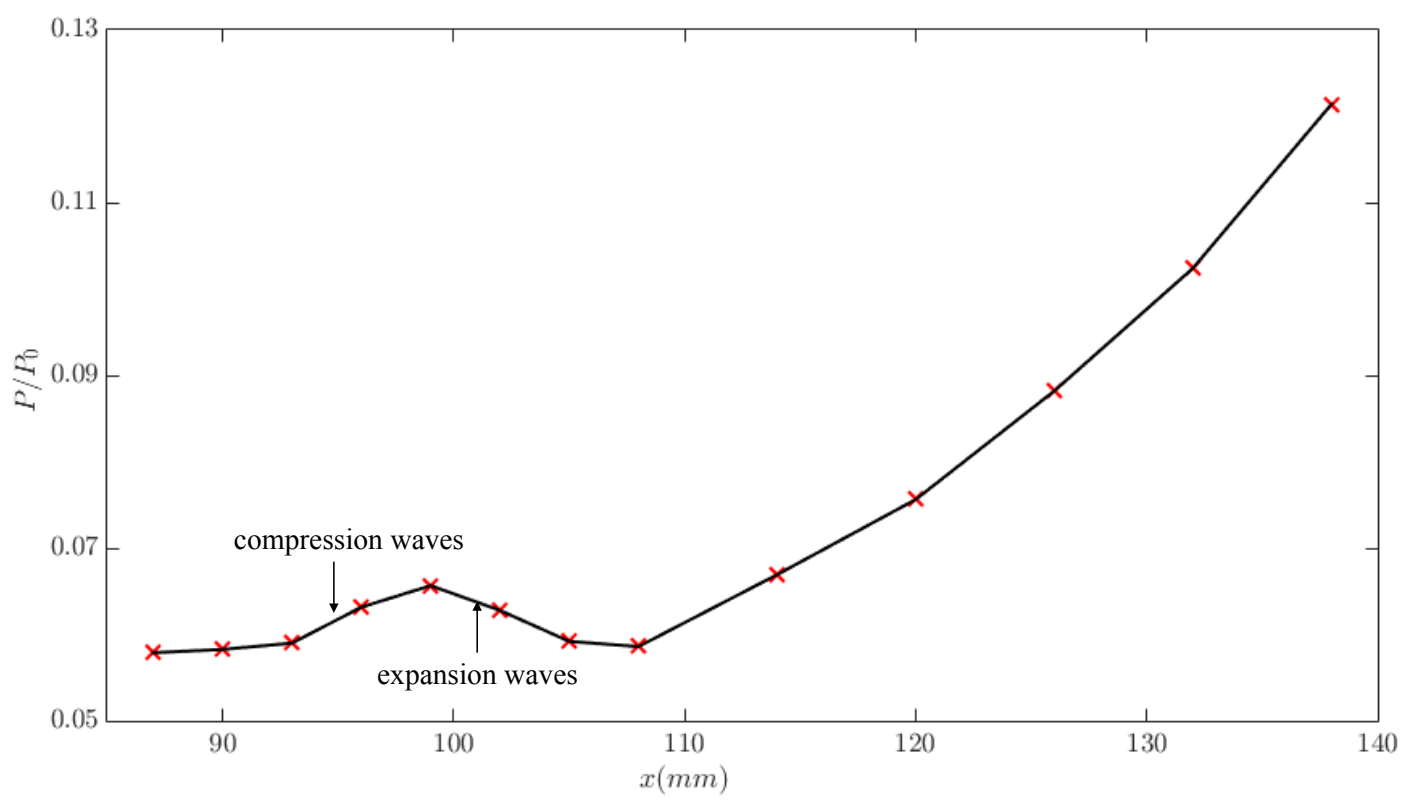

Fig. 4.9 Structure of the wave pattern induced by the corner separation illustrated by: $(a)$ PSP map on the right sidewall with extent of corner separation shown by a dotted line border; (b) static wall pressure distribution measured by pressure tappings at $z=57 \mathrm{~mm}, y=20 \mathrm{~mm}$.

The PSP map on the right sidewall is shown in figure 4.9a. Here, no quantitative data can be extracted due to a combination of more prominent image noise (poor UV light access) and thermal effects. The approximate onset and extent of corner separation, as identified from figure 4.8, is also indicated by a dotted line. The wall pressure measured with taps located in $3 \mathrm{~mm}$ intervals along the white-dashed line at $y=20 \mathrm{~mm}$ is shown in figure $4.9 \mathrm{~b}$. It is observed that the pressure near the onset of corner separation first increases and then reduces again. This suggests that the wave pattern, caused by the corner separation, consists of a compression fan followed by expansion waves of equal strength. The presence and strength 
of the expansion waves have been overlooked in previous studies [28, 51]. Downstream of the corner waves, the sidewall pressure is seen to rise rapidly across the oblique SBLI.

Figure 4.10a schematically illustrates the waves induced by corner separation. It is proposed that the separation acts like an aerodynamic bump, which first deflects the supersonic flow away from the corner, generating compression waves, and then returns the flow towards the streamwise direction, which is the cause of the observed expansion waves. The fact that such expansion waves are not visible on the tunnel floor is primarily due to a further downstream position of the corner separation 'crest' compared to that of the sidewall. In the near-wall region of the tunnel floor $x_{\text {crest }}=130 \mathrm{~mm}$ is identified from figure 4.6; while figure $4.9 \mathrm{a}$ shows a location of $x_{\text {crest }}=100 \mathrm{~mm}$ for the sidewall corner. The expansion fan induced over the convex bump is therefore located within the SBLI zone on the channel floor, with its effect being smeared-out by the stronger waves of the main interaction.

For further analysis of corner effects, the pattern of these waves generated by corner separation is traced. Of particular interest is the location of the most upstream leading compression wave along the floor. The PSP image on the tunnel floor (figure 4.6) shows this wave very clearly in the region ahead of the SBLI. Once the waves enters interaction zone its footprint is however no longer easily identifiable. It is expected that the corner waves will change direction as they penetrate into non-uniform regions and interact with other waves. However, these changes of direction are relatively small. Therefore, a rough approach to determine the upstream boundary of the corner influence region is to define a 'corner shock footprint' which combines the leading compression into a single line. The location and direction of this line is determined from the PSP map on the floor and, for simplicity, the footprint is assumed to follow a straight line across the interaction domain (until the central-span is reached where it will cross with the equivalent wave from the opposite corner).

When this footprint is applied to the oil-flow image, as shown in figure $4.10 \mathrm{~b}$, it is observed that the lines generally pass through the point where inflow skin friction lines near the sides of the tunnel are initially deflected towards the centre by the displacement effect of corner separation. It is also noteworthy that the corner shock footprints cross the two focal points bounding the centreline separation, and that the region in-between the corner waves is characterised by relatively straight separation and reattachment lines, confirming that it is outside the domain influenced by corner effects. In the central symmetry plane, the corner shocks and following expansion waves are seen to arrive well behind the interaction domain, with the streamwise intersection point of the corner shocks estimated to be at $x=160 \mathrm{~mm}$. 


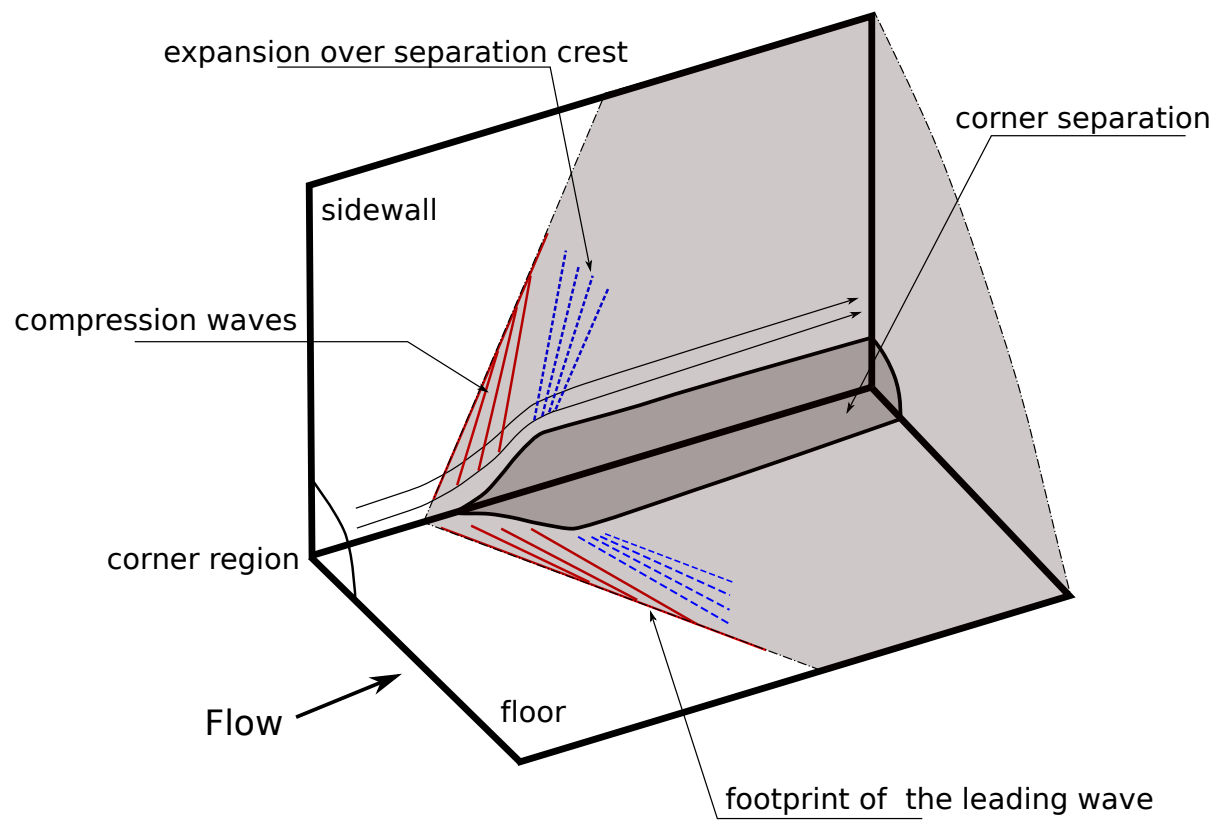

(a)

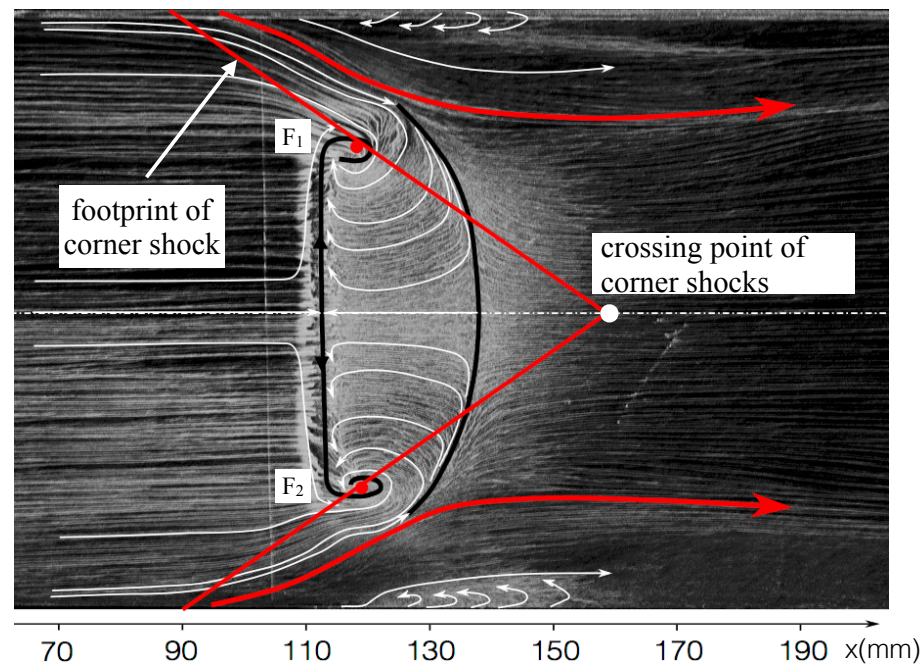

(b)

Fig. 4.10 Illustration of corner waves on baseline flow: (a) schematic diagram of corner wave structure; $(b)$ corner shock footprint indicated on oil-flow image. 


\subsection{Interaction with reduced corner separations}

The argument stated above makes it reasonable to think that different positions of the corner shock relative to the main incident shock can alter the initially two-dimensional SBLI. This can be achieved by modifying the onset and magnitude of corner separations. To examine the effect of a reduced corner separation, control in the forms of localised corner suction and micro-VGs are tested.

\subsubsection{Interaction in the presence of corner suction}

Figure 4.11 shows schlieren visualisation for the cases controlled by corner suction at three different streamwise locations upstream, across and downstream of the oblique interaction. An additional expansion fan is observed to originate from the leading edge of the suction slot, where low momentum fluid turns into the bleed slot. Otherwise, there is no prominent difference from the baseline interaction (figure 4.1). This suggests the main SBLI is not affected directly by the presence of corner suction.

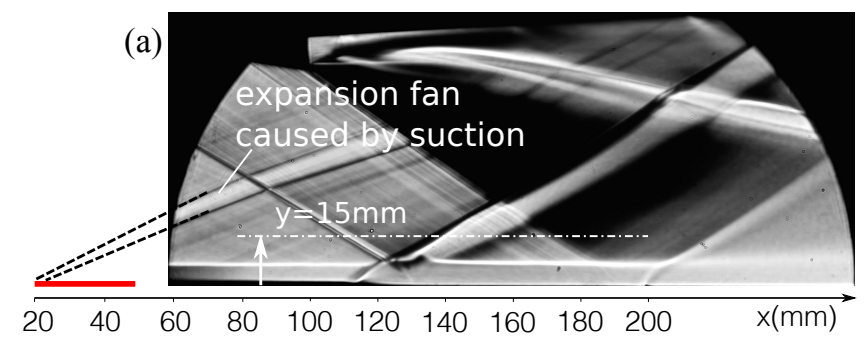

(b)
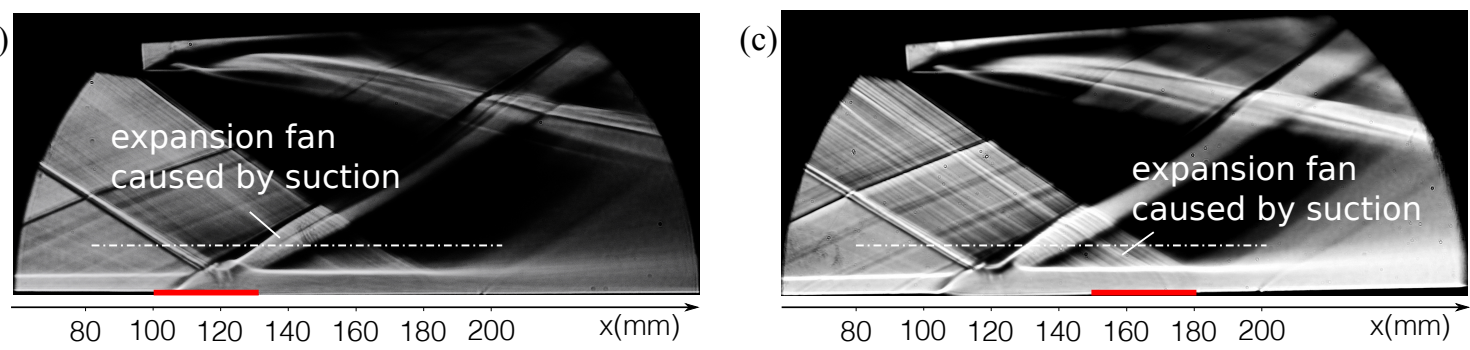

Fig. 4.11 Schlieren photographs with corner suction applied at: $(a) x_{\text {slot }}=20 \mathrm{~mm} ;(b)$ $x_{\text {slot }}=100 \mathrm{~mm} ;(c) x_{\text {slot }}=150 \mathrm{~mm}$.

Figure 4.12a provides the oil-flow visualisation with suction applied across the interaction zone at $x_{\text {slot }}=100 \mathrm{~mm}$. With a portion of low-momentum flow removed from the corner region, the corner interaction has reduced in size. The left-hand corner separation now spans $16 \mathrm{~mm}$, which is a $27 \%$ decrease compared to the baseline interaction. A similar reduction to a $19 \mathrm{~mm}$ spanwise distance is seen in the right-hand corner. It is also noted that the 
(a)

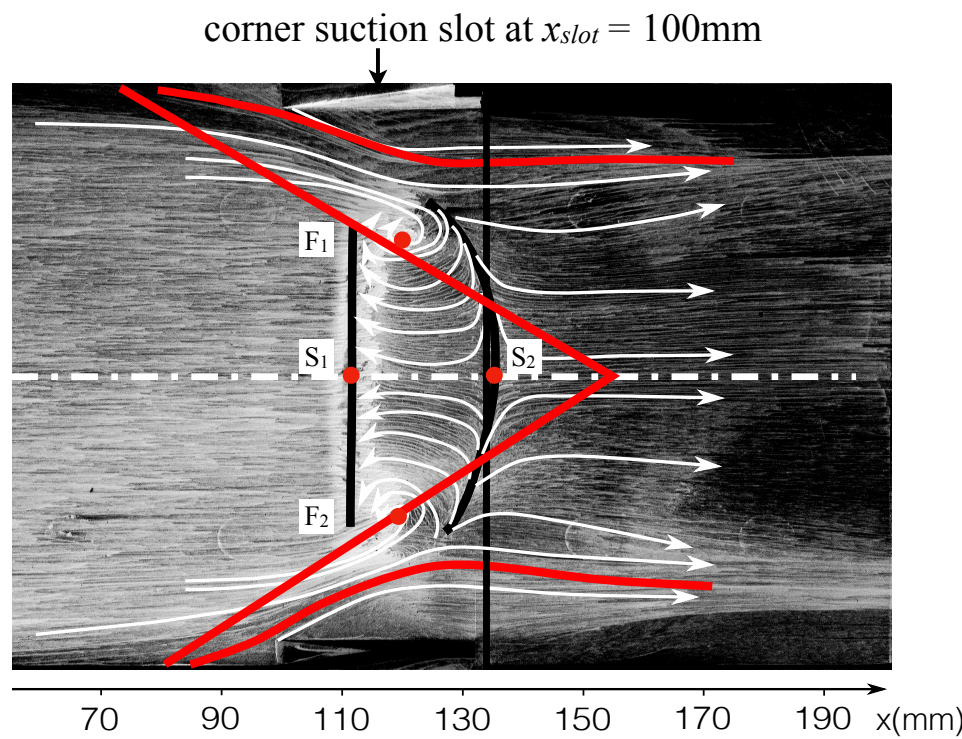

(b)

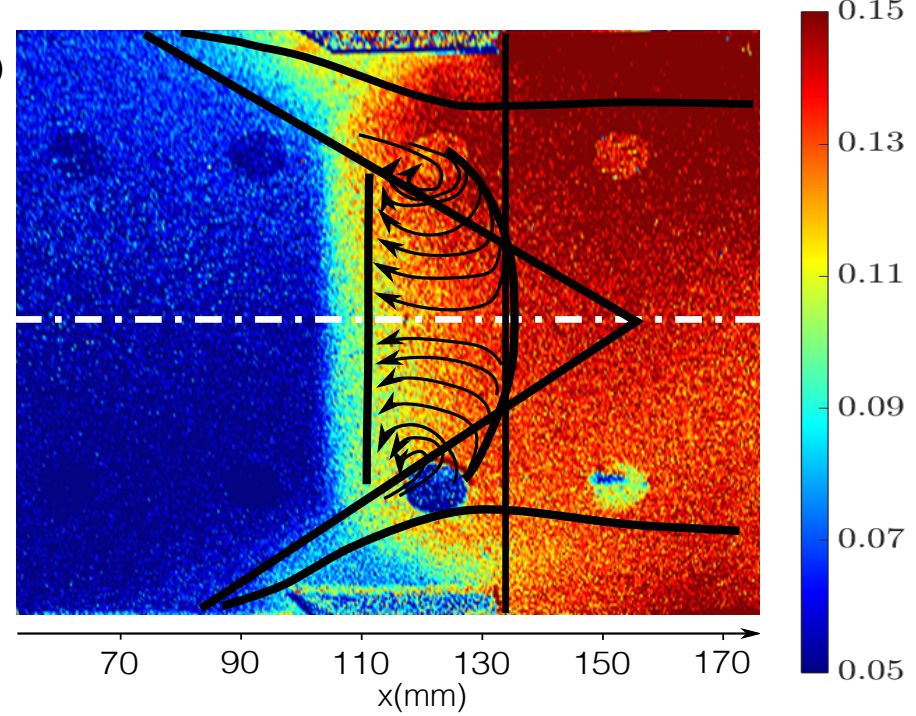

Fig. 4.12 Surface flow visualisation on the tunnel floor for the flow controlled by corner suction at $x_{\text {slot }}=100 \mathrm{~mm}$ : $(a)$ oil-flow image; $(b)$ PSP map.

onset of corner separation has moved further upstream, being around $32 \mathrm{~mm}$ ahead of the centreline separation line. Despite the changes in the corner region, the central separation displays a similar oil-flow pattern to the baseline flow, covering a streamwise distance $L_{s e p}$ of $25 \mathrm{~mm}$ and a spawise extent $L_{f}$ of $58 \mathrm{~mm}$. The attached channel between the central and corner separation is observed to be slightly wider than in the uncontrolled flow. The central separation topology can still be classified as an 'owl-face of the first kind'.

Figure $4.12 \mathrm{~b}$ presents the pressure distribution. The smearing of the pressure rise in the corner starts further upstream, which is in accordance with the earlier onset of corner 
separation. The approximate corner shock footprint traced by the PSP map suggests that the waves cross at $x=156 \mathrm{~mm}, 4 \mathrm{~mm}$ upstream of the baseline interaction crossing point.

Figure 4.13 shows the surface streamline pattern with bleed placed further downstream at $x_{\text {slot }}=150 \mathrm{~mm}$. The corner separation extents are reduced to $18 \mathrm{~mm}$ and $17 \mathrm{~mm}$ for the left and right-hand corners respectively. This shows that suction located downstream is equally capable as that across the main interaction in terms of reducing corner separations. The

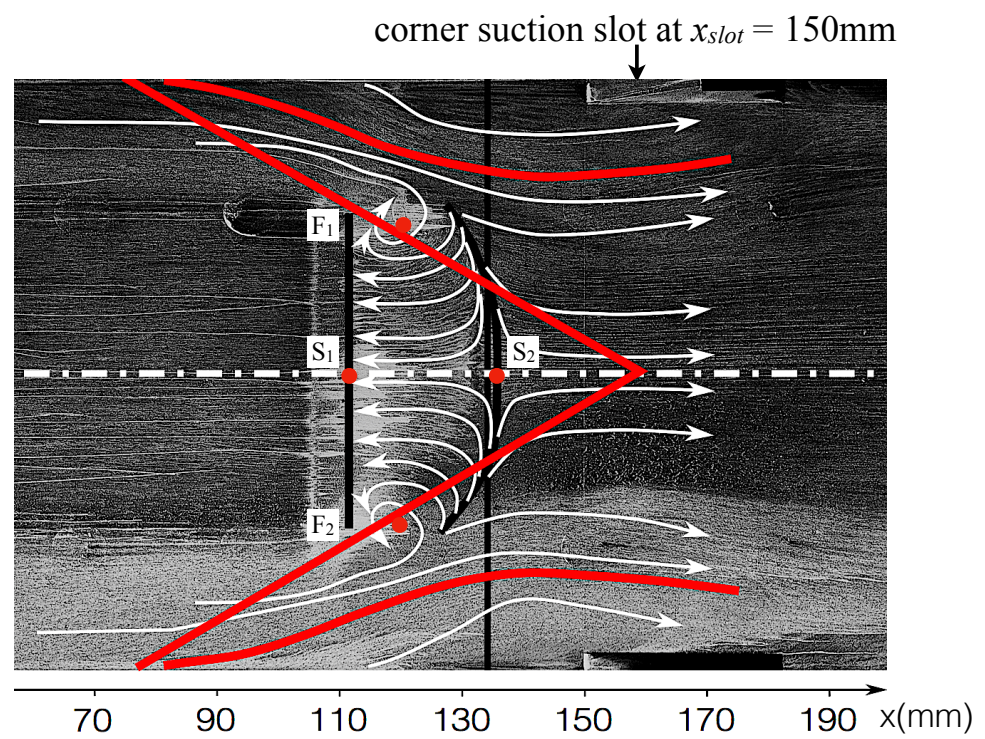

Fig. 4.13 Oil-flow visualisation on the tunnel floor for the flow controlled by corner suction at $x_{\text {slot }}=150 \mathrm{~mm}$.

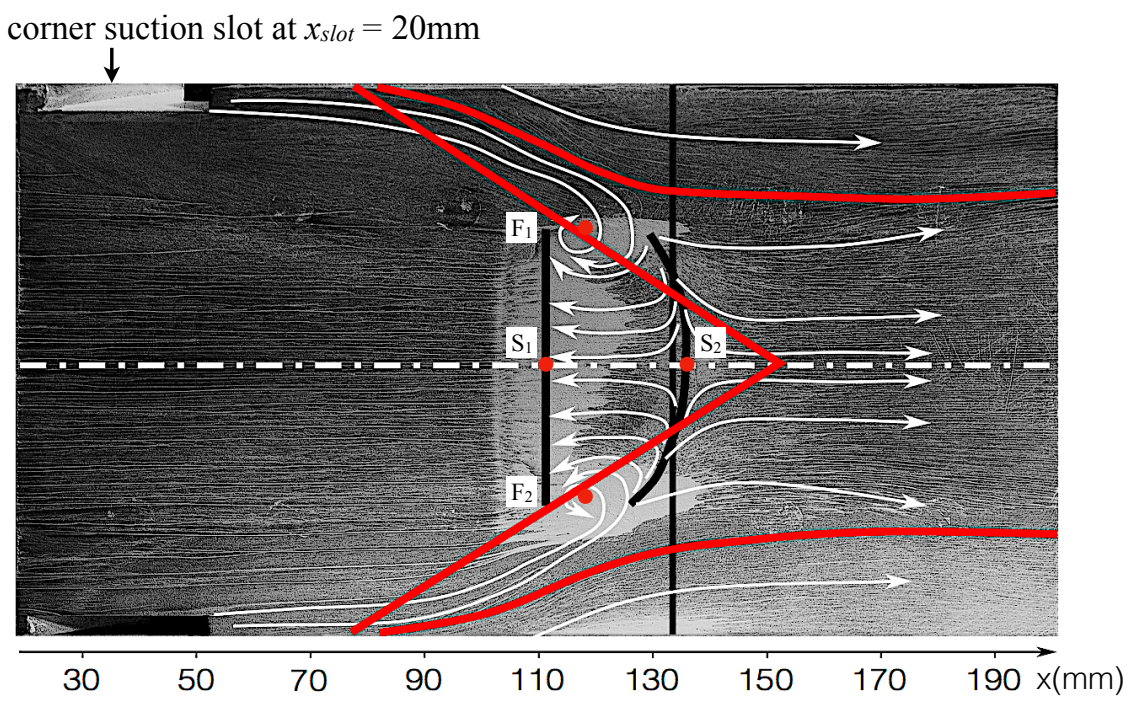

Fig. 4.14 Oil-flow visualisation on the tunnel floor for the flow controlled by corner suction at $x_{\text {slot }}=20 \mathrm{~mm}$. 
centreline separation remains almost the same as in the baseline flow, covering a streamwise length of $25 \mathrm{~mm}$ and a $60 \mathrm{~mm}$ span. Here the crossing point of the corner shocks locates at $x=160 \mathrm{~mm}$.

Upstream bleed located at $x_{\text {slot }}=20 \mathrm{~mm}$, as shown in figure 4.14 , is however less effective at controlling the corner flow. Here the separations in both of the corners are only reduced by $9 \%$ from the baseline case, covering a 20mm spanwise distance. The separation developed along the centreline is observed to be more three-dimensional, with the reattachment line curving more than in any of the previous tests. The increase of three-dimensionality is accompanied by the intersection point of the corner shocks shifting forward to $x=153 \mathrm{~mm}$. The central separation now spans $56 \mathrm{~mm}$, which is a $10 \%$ decrease in the quasi-two-dimensional part of the main SBLI compared to the unmodified flow.

As with the case of $x_{\text {slot }}=100 \mathrm{~mm}$, both downstream and upstream bleed somehow move the onset of corner separations further upstream, starting approximately $30 \mathrm{~mm}$ earlier than the centreline separation.

Table 4.1 summarises the control effectiveness of corner suction. The results indicate that bleed placed downstream and across the interaction are better than upstream slots at suppressing oblique shock-induced separations in corners. However, localised corner suction, even at a favourable control position, can only mitigate the corner interactions so mildly that the central separation is barely affected. Despite some success in reducing the size of corner separations, the onset of local separation is also somehow surprisingly moved further upstream. These two effects cancel each other, the approximate position of the corner shock crossing point is therefore barely changed from the baseline case. The three-dimensional flow structures are seen to be very similar in uncontrolled case and flows controlled by corner bleed. More control methods are hence discussed in the following sections, aiming to achieve a greater change on corner separations and hence the location of corner shocks.

Table 4.1 Summary for the control effectiveness of corner-bleed (in mm).

\begin{tabular}{lcccc}
\hline Experiment & $\begin{array}{c}\text { left-hand } \\
\text { corner sep. }\end{array}$ & $\begin{array}{c}\text { right-hand } \\
\text { corner sep. }\end{array}$ & $\begin{array}{c}\text { averaged reduction rate } \\
\text { to baseline }(\%)\end{array}$ & $\begin{array}{c}\text { bleed rate } \\
\%_{\dot{m}_{\text {total }}}\end{array}$ \\
\hline Baseline & 21 & 23 & 0 & 0 \\
$x_{\text {slot }}=20 \mathrm{~mm}$ & 20 & 20 & 9 & 0.21 \\
$x_{\text {slot }}=100 \mathrm{~mm}$ & 16 & 19 & 18 & 0.28 \\
$x_{\text {slot }}=150 \mathrm{~mm}$ & 18 & 17 & 18 & 0.41 \\
\hline
\end{tabular}




\subsubsection{Interaction in the presence of micro-VGs within the corners}

Figure 4.15 shows a schlieren image of the flow field controlled by two $3 \mathrm{~mm}$ micro-VGs located at $x_{v g}=50 \mathrm{~mm}$. The main separated oblique SBLI wave pattern is almost the same as that of the baseline flow. Two oblique shock waves, faint however still visible, are seen to emanate from the leading and trailing edges of the vanes.

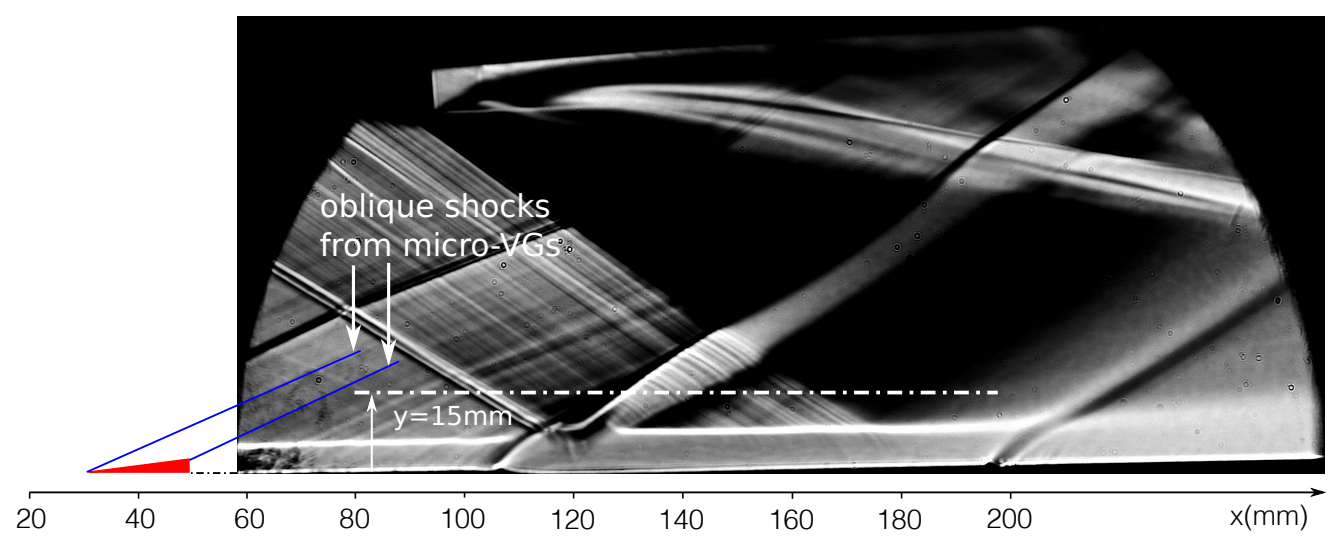

Fig. 4.15 Schlieren photograph with micro-VGs applied at $x_{v g}=50 \mathrm{~mm}$.

The tunnel floor oil streak image is displayed in figure 4.16. A region of comparably high shear stress is identified in the wake of the micro-VGs. This is the footprint of the primary vortex generated by the trailing edge of the vane. The corner separations are reduced in size by approximately $45 \%$ compared to the baseline interaction, with the distances extending

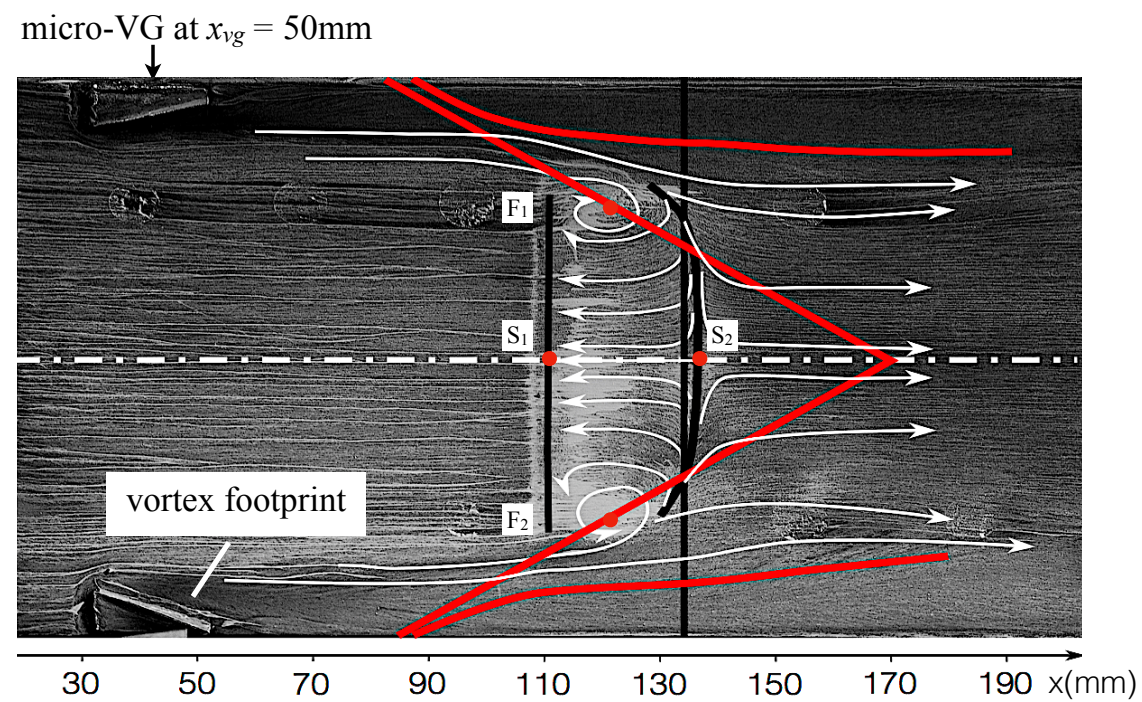

Fig. 4.16 Oil-flow visualisation on the tunnel floor for the flow controlled by micro-VGs at $x_{v g}=50 \mathrm{~mm}$. 

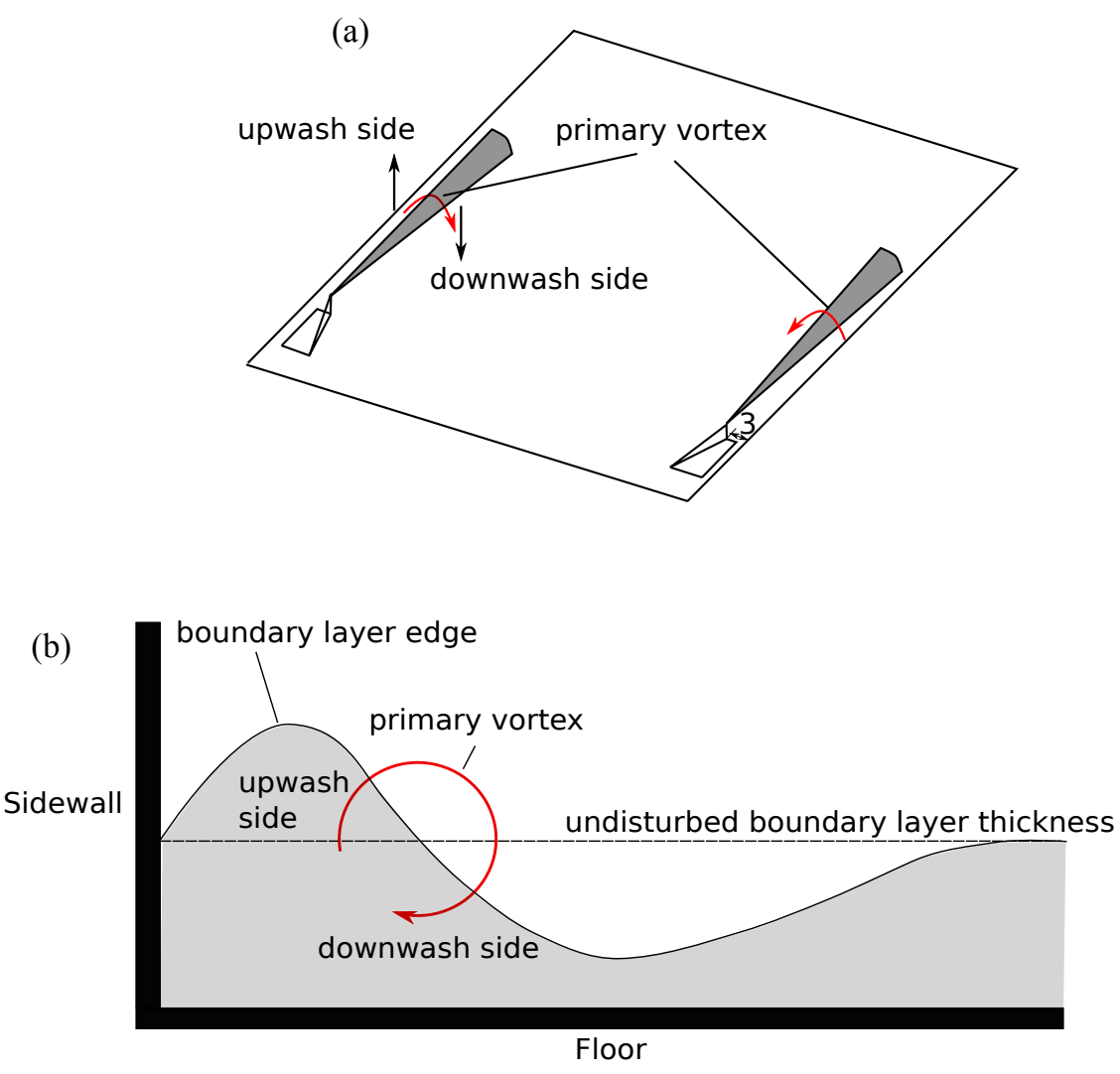

Fig. 4.17 Schematic illustration for flow control mechanism of micro-VGs within the corner: $(a)$ vortices produced by the micro-vane $(b)$ streamwise velocity contour downstream of VG.

from the sidewall being $12 \mathrm{~mm}$ for the left-hand corner and $13 \mathrm{~mm}$ for the right-hand. A more two-dimensional flow is observed, as evidenced by the increased distance between the two focal points of $L_{f}=66 \mathrm{~mm}$ (a $6 \%$ increase from the uncontrolled flow). The streamwise separation length along the mid-span symmetry line is however not affected by the presence of VGs remaining unchanged $L_{s e p}=25 \mathrm{~mm}$. The corner shocks generated by the reduced corner separations now locate further away from the centre of the wind tunnel. It can be seen that the two shock waves cross at $\mathrm{x}=171 \mathrm{~mm}, 11 \mathrm{~mm}$ downstream from the baseline case.

Figure 4.17 illustrates schematically the proposed flow-control mechanism with microVGs employed in the corner region. The vortices generated by the VGs propagate downstream, creating upwash near the sidewall and downwash with entrainment of high-energy flow on the side facing the tunnel centreline. Low-momentum fluid is now swept into the near-wall region, while for flow further away from the sidewall a thinner and healthier boundary layer than in the uncontrolled case develops. Futher downstream, towards the main interaction, the low-energy wake of VGs is moved up and eventually ejected from the boundary layer due to the upwash effect. The boundary layer in the corners is therefore 
conditioned to be more resistant to the shock-induced APG. As discussed in section 3.2.1, the micro-VG angle is set to $15^{\circ}$ to produce a peak circulation to an inflow of $M=2.5$. The device location relative to the main interaction, $14 \delta$, is sufficiently far upstream for the vortices to bring about beneficial boundary layer control, while not too far so that the vortices lift off the surface and break down ahead of the separation.

\subsubsection{Interaction with combined micro-VGs and corner suction}

Figure 4.18 shows a schlieren image where a combination of micro-VGs $\left(x_{v g}=50 \mathrm{~mm}\right)$ and corner suction $\left(x_{\text {slot }}=100 \mathrm{~mm}\right)$ has been used to reduce the size of corner separation. Once again, no prominent difference from the baseline flow can be observed except for some evidence of an expansion fan originating from the suction slots and the oblique shock wave created by the edges of micro-VGs.

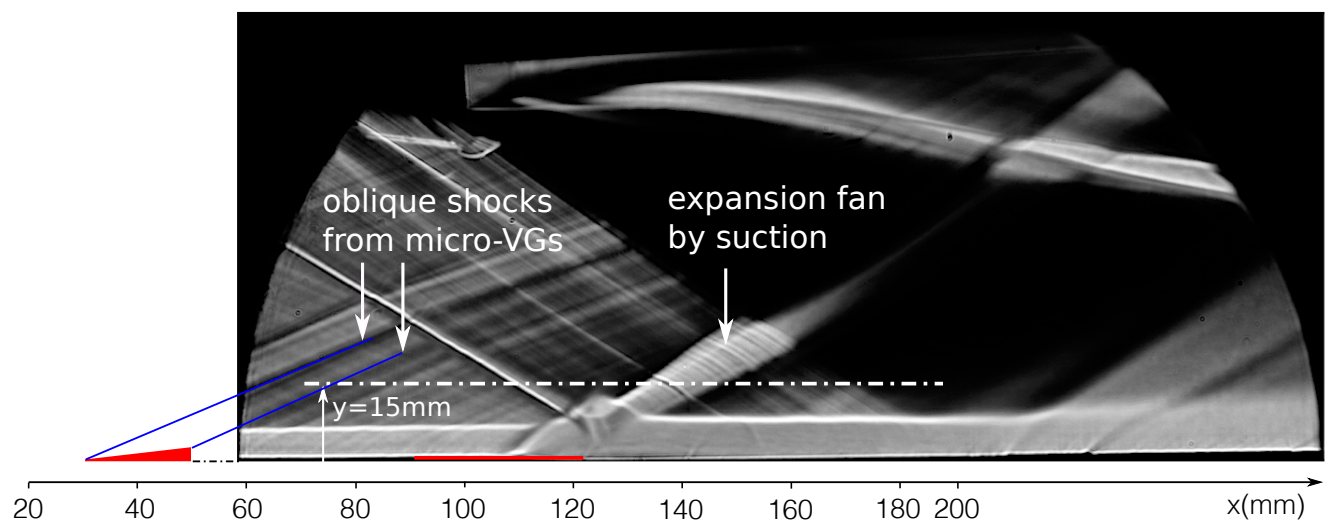

Fig. 4.18 Schlieren photograph with micro-VGs $\left(x_{v g}=50 \mathrm{~mm}\right)$ and corner suction $\left(x_{\text {slot }}=\right.$ $100 \mathrm{~mm}$ ) employed in tandem.

Figure 4.19a displays the oil-flow visualisation on the tunnel floor. It can be seen that the corner separations are considerably reduced, spanning only $8 \mathrm{~mm}$, which is a $65 \%$ decrease from the baseline. The corner separation onset is located approximately $32 \mathrm{~mm}$ ahead of the centreline separation line. The three-dimensionality of the surface separation topology is further reduced compared to the oblique SBLI with micro-VG only control. The spanwise extent of the central separation $L_{f}$ is now $72 \mathrm{~mm}$ ( $16 \%$ greater than that of baseline) while $L_{\text {sep }}$ remains unchanged at $25 \mathrm{~mm}$. The effect of control is also reflected in the surface pressure distribution presented in figure $4.19 \mathrm{~b}$, which exhibits a wider region with little spanwise variation at the start of the interaction and reduced pressure smearing near the sidewalls. The corner shock location, determined from the PSP map, shows that the waves 
arrive at the central symmetry plane at $x=181 \mathrm{~mm}, 21 \mathrm{~mm}$ downstream from the uncontrolled flow.

(a)

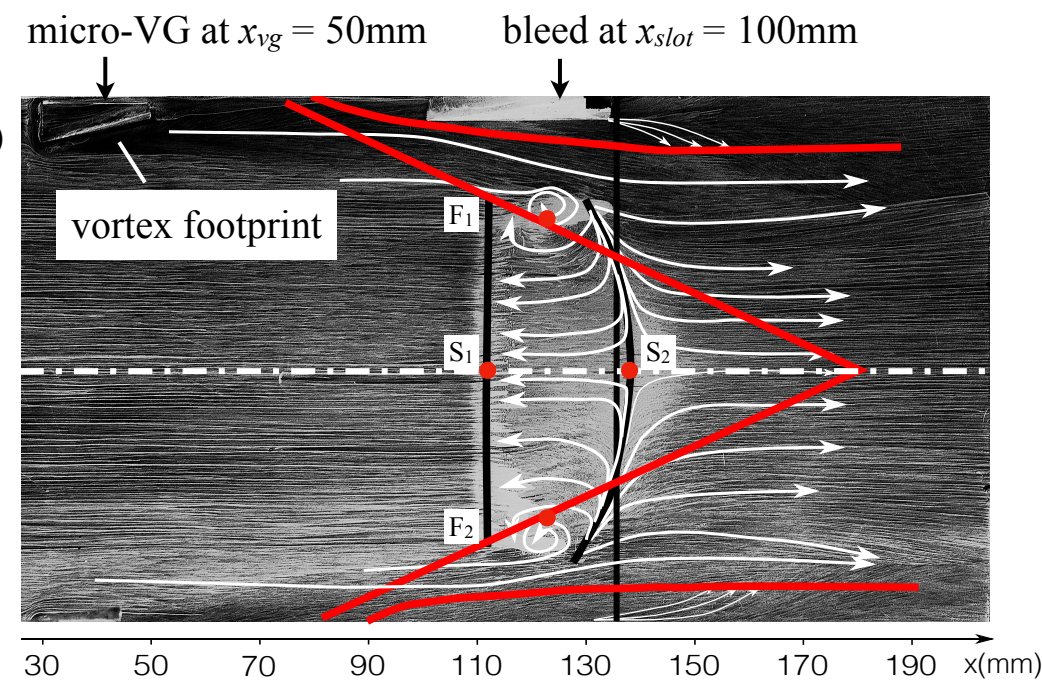

(b)

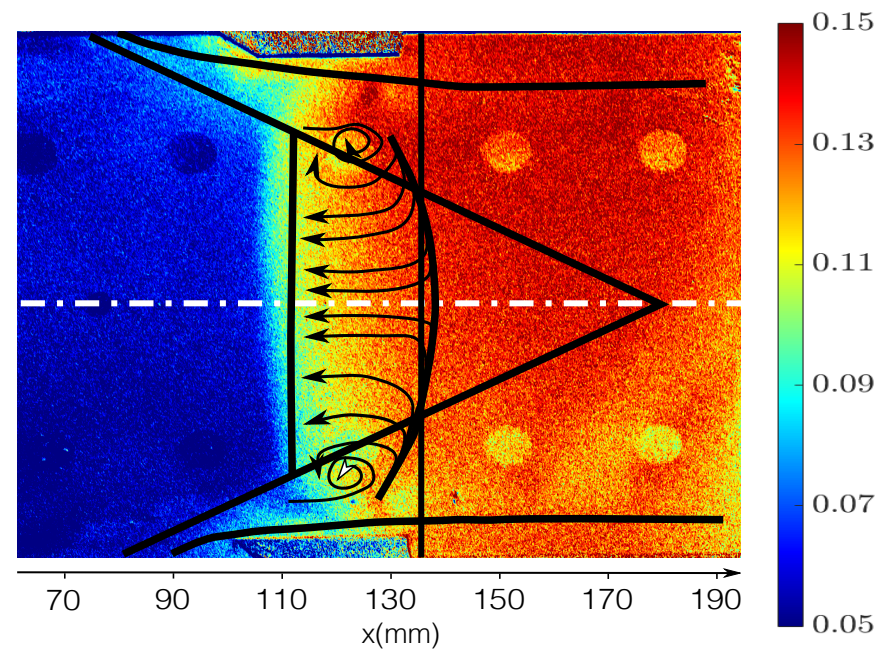

Fig. 4.19 Surface flow visualisation on the tunnel floor for the flow with combined control applied: $(a)$ oil-flow image; $(b)$ PSP map.

Table 4.2 summarises the relevant geometric measurements for all cases discussed so far. It can be seen that the combination of micro-VGs and bleed works most effectively to control corner separation. Vane-type micro-VGs reduce the size of corner separation to a lesser however still considerable extent, whilst suction-only control imposes a slight change. The streamwise length in the centre $L_{\text {sep }}$ remains unchanged for all the tests, indicating that neither the incoming flow nor the strength of the main interaction along the mid-span symmetry line is influenced by the corner flow treatments. This finding is confirmed by the boundary layer parameters on the tunnel floor centreline of the inflow and flow downstream of 
Table 4.2 Summary of separation-related parameters for oblique SBLIs with reduced corner separations (in $\mathrm{mm}$ ).

\begin{tabular}{lccccc}
\hline Experiment & $L_{\text {sep }}$ & $L_{f}$ & $\begin{array}{c}\text { left-corner } \\
\text { sep. }\end{array}$ & $\begin{array}{c}\text { right-corner } \\
\text { sep. }\end{array}$ & $\begin{array}{c}\text { corner shocks } \\
\text { crossing point }\end{array}$ \\
\hline Baseline & 25 & 62 & 21 & 23 & $x=160 \mathrm{~mm}$ \\
$x_{\text {slot }}=20 \mathrm{~mm}$ & 25 & 56 & 20 & 20 & $x=153 \mathrm{~mm}$ \\
$x_{\text {slot }}=100 \mathrm{~mm}$ & 25 & 58 & 16 & 19 & $x=156 \mathrm{~mm}$ \\
$x_{\text {slot }}=150 \mathrm{~mm}$ & 25 & 60 & 18 & 17 & $x=160 \mathrm{~mm}$ \\
$x_{v g}=50 \mathrm{~mm}$ & 25 & 66 & 12 & 13 & $x=171 \mathrm{~mm}$ \\
$x_{v g}=50 \mathrm{~mm}$ and $x_{\text {slot }}=100 \mathrm{~mm}$ & 25 & 72 & 8 & 8 & $x=181 \mathrm{~mm}$ \\
\hline
\end{tabular}

the oblique SBLI, as listed in table 4.3 and 4.4, where little difference among the experiments is detected.

Table 4.3 Boundary layer parameters at $x=80 \mathrm{~mm}$ along the tunnel floor centreline for baseline and experiments with reduced corner separations.

\begin{tabular}{lcccc}
\hline Experiment & $\delta(\mathrm{mm})$ & $\delta^{*}(\mathrm{~mm})$ & $\theta(\mathrm{mm})$ & $\mathrm{H}$ \\
\hline Baseline & 6.1 & 0.84 & 0.61 & 1.37 \\
$x_{\text {slot }}=20 \mathrm{~mm}$ & 6.2 & 0.82 & 0.60 & 1.36 \\
$x_{\text {slot }}=100 \mathrm{~mm}$ & 6.1 & 0.84 & 0.61 & 1.37 \\
$x_{\text {slot }}=150 \mathrm{~mm}$ & 6.2 & 0.82 & 0.60 & 1.36 \\
$x_{v g}=50 \mathrm{~mm}$ & 6.1 & 0.82 & 0.60 & 1.36 \\
$x_{v g}=50 \mathrm{~mm}$ and $x_{\text {slot }}=100 \mathrm{~mm}$ & 6.1 & 0.85 & 0.62 & 1.38 \\
\hline
\end{tabular}

Table 4.4 Boundary layer parameters at $x=160 \mathrm{~mm}$ along the tunnel floor centreline for baseline and experiments with reduced corner separations.

\begin{tabular}{lcccc}
\hline Experiment & $\delta(\mathrm{mm})$ & $\delta^{*}(\mathrm{~mm})$ & $\theta(\mathrm{mm})$ & $\mathrm{H}$ \\
\hline Baseline & 7.6 & 2.14 & 1.17 & 1.83 \\
$x_{\text {slot }}=20 \mathrm{~mm}$ & 7.5 & 2.10 & 1.17 & 1.81 \\
$x_{\text {slot }}=100 \mathrm{~mm}$ & 7.4 & 1.98 & 1.10 & 1.80 \\
$x_{\text {slot }}=150 \mathrm{~mm}$ & 7.6 & 2.12 & 1.16 & 1.82 \\
$x_{v g}=50 \mathrm{~mm}$ & 7.6 & 2.16 & 1.17 & 1.84 \\
$x_{v g}=50 \mathrm{~mm}$ and $x_{\text {slot }}=100 \mathrm{~mm}$ & 7.4 & 2.06 & 1.14 & 1.82 \\
\hline
\end{tabular}




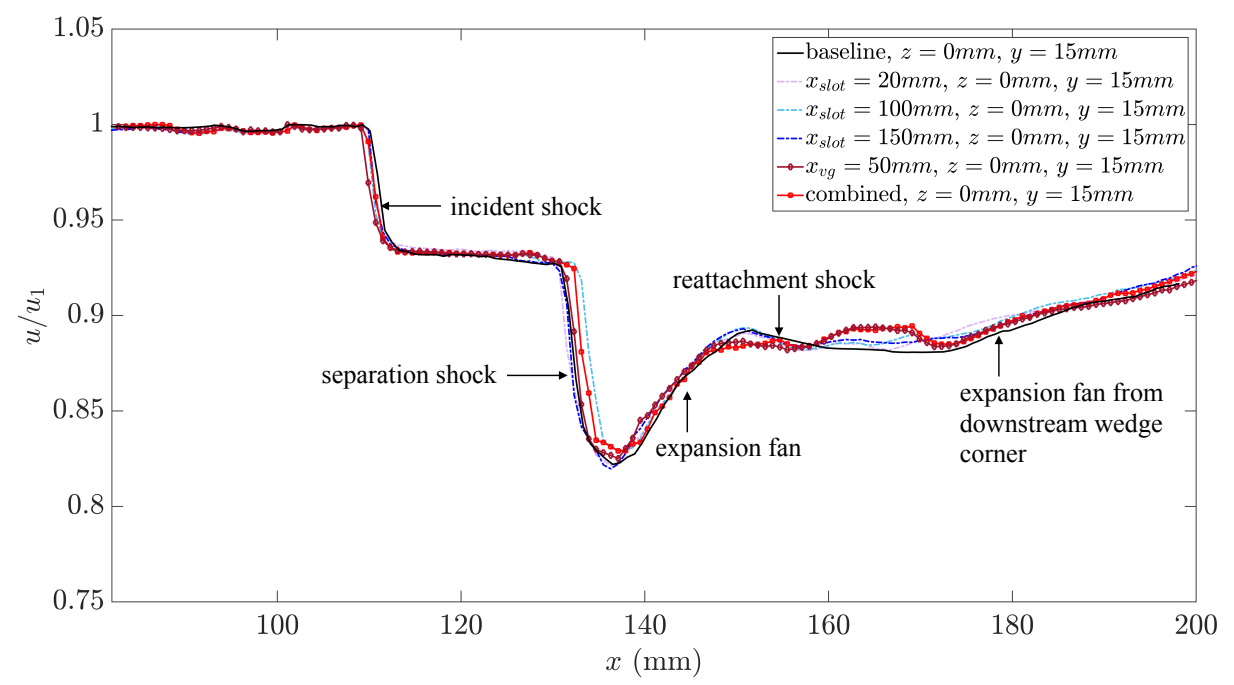

Fig. 4.20 Variation in streamwise velocity at $y=15 \mathrm{~mm}, z=0 \mathrm{~mm}$ for flows with reduced corner separations.

Figure 4.20 presents the streamwise velocity variation through the oblique SBLI above the boundary layer edge at $y=15 \mathrm{~mm}, z=0 \mathrm{~mm}$. Once again, the measurements show that the velocity variation is similar for all the configurations, confirming that corner effects did not change the flow in the central region.

The main effect of reducing the size of corner separation is therefore an increase in the quasi-two-dimensional part $\left(L_{f}\right)$ of the main SBLI.

\subsection{Interaction with increased corner separations}

It has been shown that reducing corner separations can only affect the centreline separation slightly, with no significant differences seen in the three-dimensional flow separation topology. The opposite effect of increasing corner separations is therefore explored.

Two corner obstructions, consisting of blocks with dimensions $6 \times 6 \times 20 \mathrm{~mm}^{3}$, are tested first in section 4.3.1. Further experiments with larger obstacles, sized $10 \mathrm{~mm}$ for the width and height respectively, are performed to increase the magnitude of corner separations more considerably, and the results are presented in section 4.3.2. 


\subsubsection{Interaction in the presence of small block}

Figure 4.21 displays the schlieren photograph of flow field modified by two $6 \times 6 \times 20 \mathrm{~mm}^{3}$ corner blocks placed across the interaction domain at $x_{\text {block }}=105 \mathrm{~mm}$. As with interactions with reduced corner separations, the main oblique SBLI wave pattern exhibits no prominent difference from the baseline flow. A faint oblique shock wave is seen to emanate from the leading edge of the blocks while an expansion fan from the rear.

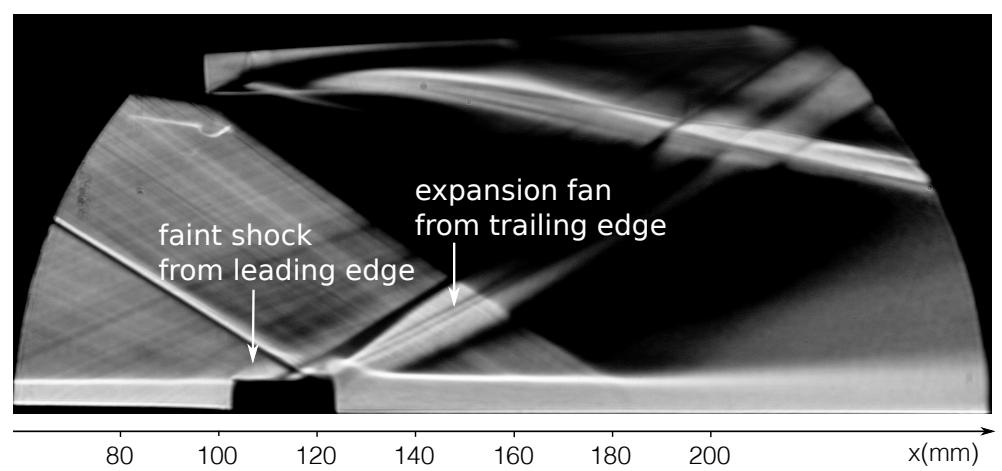

Fig. 4.21 Schlieren photograph with small corner obstacle introduced at $x_{\text {block }}=105 \mathrm{~mm}$.

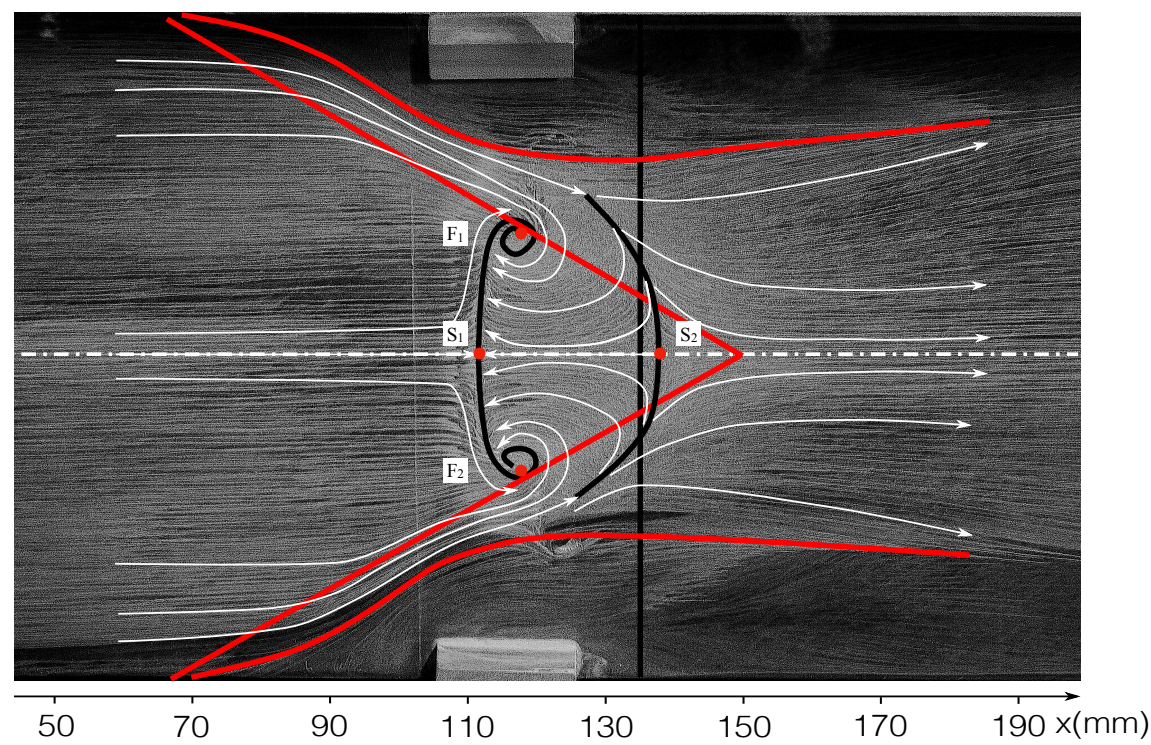

Fig. 4.22 Oil-flow visualisation on the tunnel floor for the flow disturbed by small corner blocks at $x_{\text {block }}=105 \mathrm{~mm}$.

Figure 4.22 shows the oil-flow image on the tunnel floor. It can be seen that with the corner blocks applied the onset of corner separation is shifted forward, starting approximately $42 \mathrm{~mm}$ earlier than the centreline separation. The separations in both of the corners now extend over $26 \mathrm{~mm}$ away from the sidewall, which is an $18 \%$ increase from the unmodified interaction. 
The footprints of corner shocks are moved upstream accordingly with the intersection point located at $x=150 \mathrm{~mm}$. The most striking feature of the central separation appears to be the considerably reduced spanwise extent $L_{f}$, covering only $42 \mathrm{~mm}$ in between the two focal points at the bubble edges (a $32 \%$ decrease from the baseline case). This leads to a wider region with reduced or even vanishing separation between the corner interactions and the centre. Despite the noticeable reduction in $L_{f}$, the separation length along the centreline $L_{s e p}$ is only increased by $1 \mathrm{~mm}$. The surface streamline pattern suggests that the three-dimensional central separation is still an 'owl-face of the first kind'.

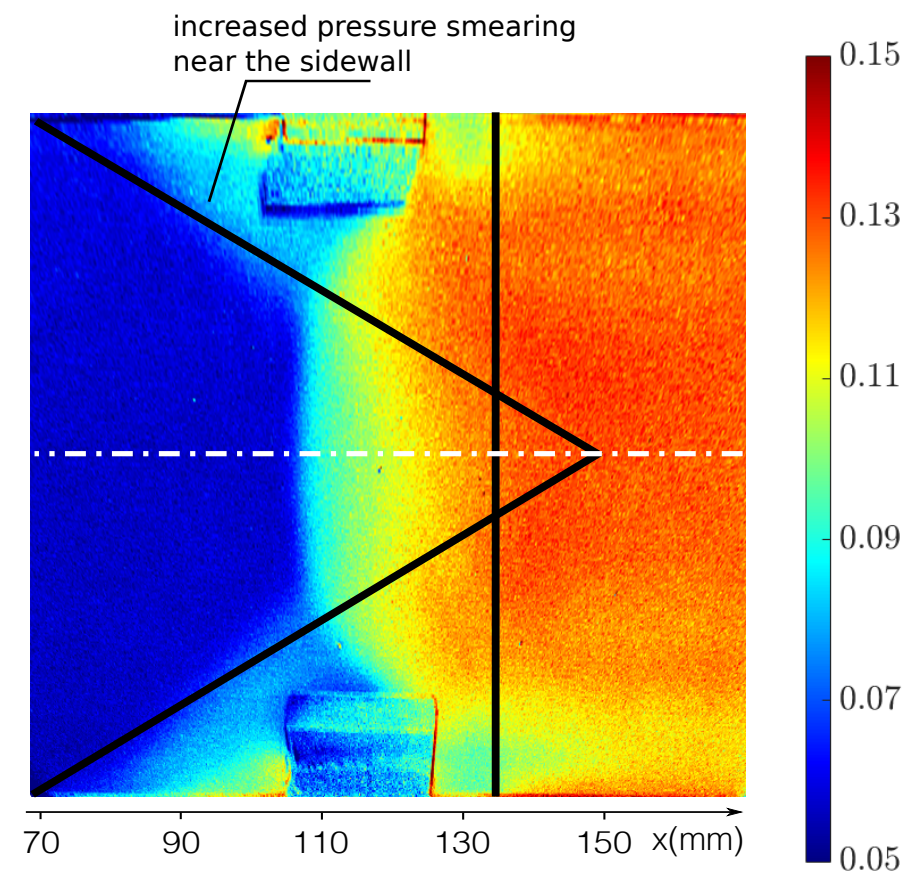

Fig. $4.23 P / P_{0}$ for the tunnel floor with small blocks at $x_{\text {block }}=105 \mathrm{~mm}$.

In figure 4.23 the static wall pressure distribution for the tunnel floor is presented. Approaching the sidewalls an increased amount of pressure smearing is observed in comparison with the baseline case (figure 4.6). In accordance with the earlier corner separations seen in the oil-flow visualisation, the pressure rise in corner regions occurs further upstream.

The pressure distribution along the tunnel floor centreline is compared with the baseline flow in figure 4.24. The corner-block case displays a slightly larger interaction length, with the pressure rise starting approximately $2 \mathrm{~mm}$ earlier. The adverse pressure gradients are however very similar, which explains the barely affected $L_{s e p}$ by the small blocks. 


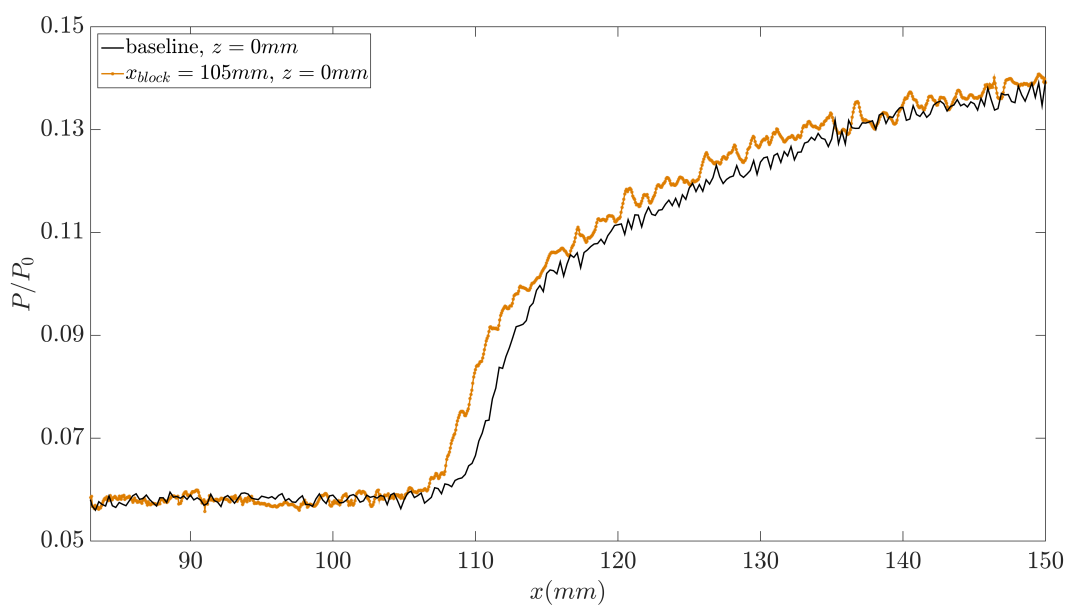

Fig. 4.24 Static wall pressure distribution on the tunnel floor centreline for small corner-block case.

\subsubsection{Interaction in the presence of large block}

Figure 4.25 shows schlieren images for two experiments where corner blocks with dimensions $10 \times 10 \times 20 \mathrm{~mm}^{3}$ are applied across the oblique interaction zone at $x_{\text {block }}=120 \mathrm{~mm}$ (figure $4.25 \mathrm{a}$ ) and $x_{\text {block }}=130 \mathrm{~mm}$ (figure $4.25 \mathrm{~b}$ ) respectively. A much larger interaction length with an onset $20 \mathrm{~mm}$ upstream of the unmodified SBLI (figure 4.1) is observed for both flow fields, and the boundary layer downstream of the interaction appears to be thicker than the baseline case. These observations suggest that for flows disturbed by the larger corner obstructions, the oblique shock-induced separation has increased in size.

In contrast, when the blocks are shifted even further upstream and located ahead of the incident shock, at $x_{\text {block }}=75 \mathrm{~mm}$ (figure $4.26 \mathrm{a}$ ) and $x_{\text {block }}=85 \mathrm{~mm}$ (figure $4.26 \mathrm{~b}$ ), the separation shock is more pronounced while the size of interaction zone exhibits no prominent difference compared to the undisturbed SBLI.

A series of oil-flow images with various corner-block positions are shown in figure 4.27. Compared to the flow disturbed by small obstacles(figure 4.22), it can be seen that for all corner block locations the corner separations are further increased in size by up to $50 \%$ than the baseline case. As the blocks move upstream the onset of corner separation shifts forward as well and the corner shock footprints (and the approximate crossing points) are found to move accordingly (as indicated by the red lines). This is accompanied by significant changes in the size and shape of centreline separation. Table 4.5 lists the parameters related to the central separation for all the corner-block cases discussed so far.

Figures 4.27(a-i) show the interactions when the corner waves intersect inside the separated region. It can be seen that the approximate footprint of the corner shocks once again cross through the focal points identified at the edges of centreline separation. In all these 

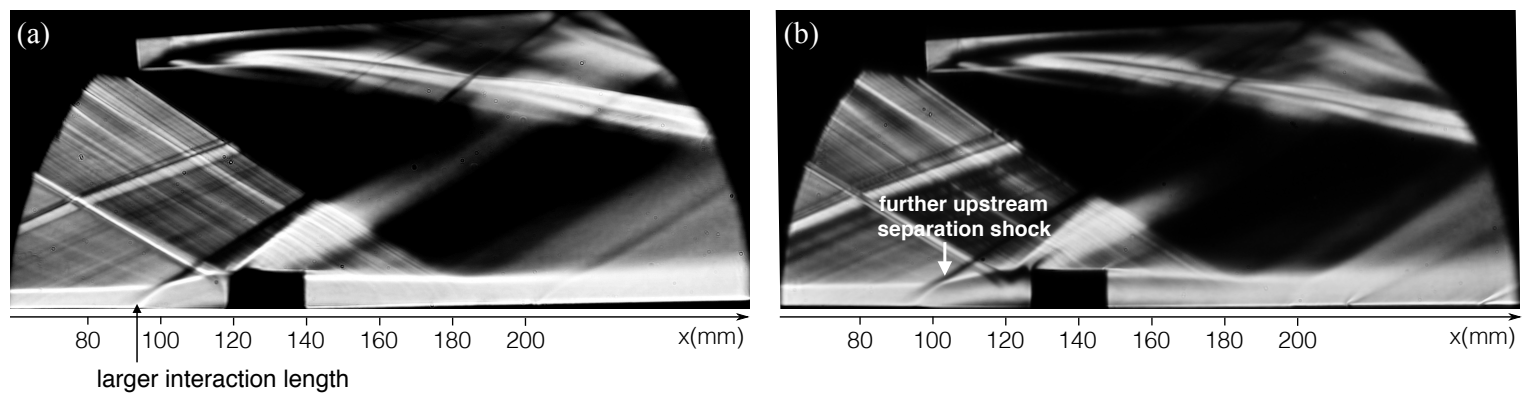

Fig. 4.25 Schlieren photographs with large corner obstacle introduced across the interaction domain: (a) $x_{\text {block }}=120 \mathrm{~mm}$; $($ b $) x_{\text {block }}=130 \mathrm{~mm}$.
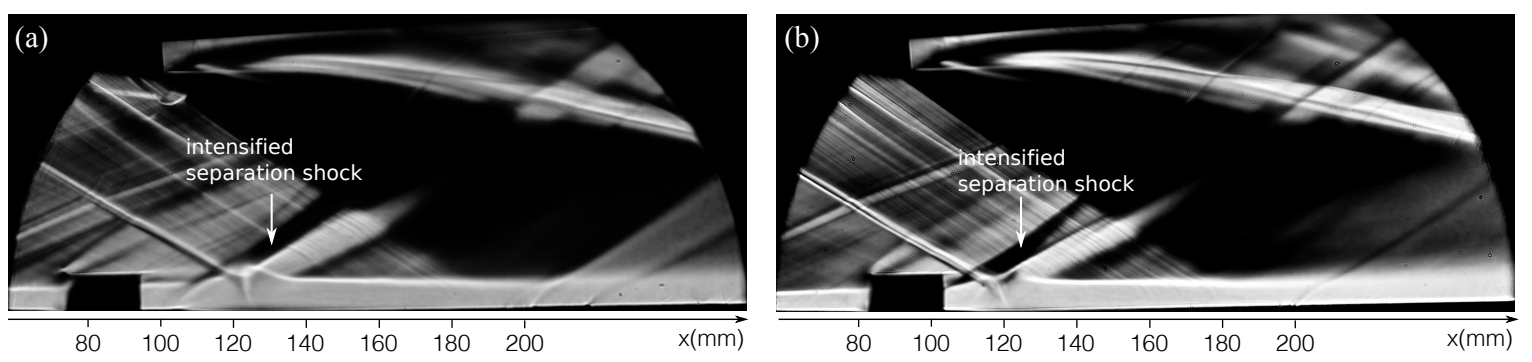

Fig. 4.26 Schlieren photographs with large corner obstacle introduced ahead of the incident shock: $(a) x_{\text {block }}=75 \mathrm{~mm} ;(b) x_{\text {block }}=85 \mathrm{~mm}$.

Table 4.5 Centre separation parameters for oblique SBLIs with increased corner separations (in $\mathrm{mm}$ ).

\begin{tabular}{lccc}
\hline Experiment & $L_{\text {sep }}$ & $L_{f}$ & corner shocks crossing point \\
\hline$x_{\text {block }}=105 \mathrm{~mm}$ (small obstacle) & 26 & 42 & $x=150 \mathrm{~mm}$ \\
$x_{\text {block }}=130 \mathrm{~mm}$ & 35 & 35 & $x=145 \mathrm{~mm}$ \\
$x_{\text {block }}=125 \mathrm{~mm}$ & 38 & 37 & $x=140 \mathrm{~mm}$ \\
$x_{\text {block }}=120 \mathrm{~mm}$ & 45 & 37 & $x=137 \mathrm{~mm}$ \\
$x_{\text {block }}=115 \mathrm{~mm}$ & 37 & 31 & $x=131 \mathrm{~mm}$ \\
$x_{\text {block }}=105 \mathrm{~mm}$ & 35 & 23 & $x=128 \mathrm{~mm}$ \\
$x_{\text {block }}=95 \mathrm{~mm}$ & 34 & 19 & $x=123 \mathrm{~mm}$ \\
$x_{\text {block }}=90 \mathrm{~mm}$ & 32 & 14 & $x=112 \mathrm{~mm}$ \\
$x_{\text {block }}=85 \mathrm{~mm}$ & 16 & 16 & $x=105 \mathrm{~mm}$ \\
$x_{\text {block }}=75 \mathrm{~mm}$ & 9 & 25 & $x=97 \mathrm{~mm}$ \\
\hline
\end{tabular}

cases, the centreline separation length $L_{s e p}$ is significantly increased. More specifically, the value of $L_{\text {sep }}$ reaches a maximum for $x_{\text {block }}=120 \mathrm{~mm}$, where the intersection point approaches the reattachment position of the unmodified SBLI at $x=137 \mathrm{~mm}$, as shown in figure 4.28a. In this case the separated flow reattaches further downstream than in any of the other tests. This is followed by a progressive decrease in $L_{\text {sep }}$ as the corner shock intersection 


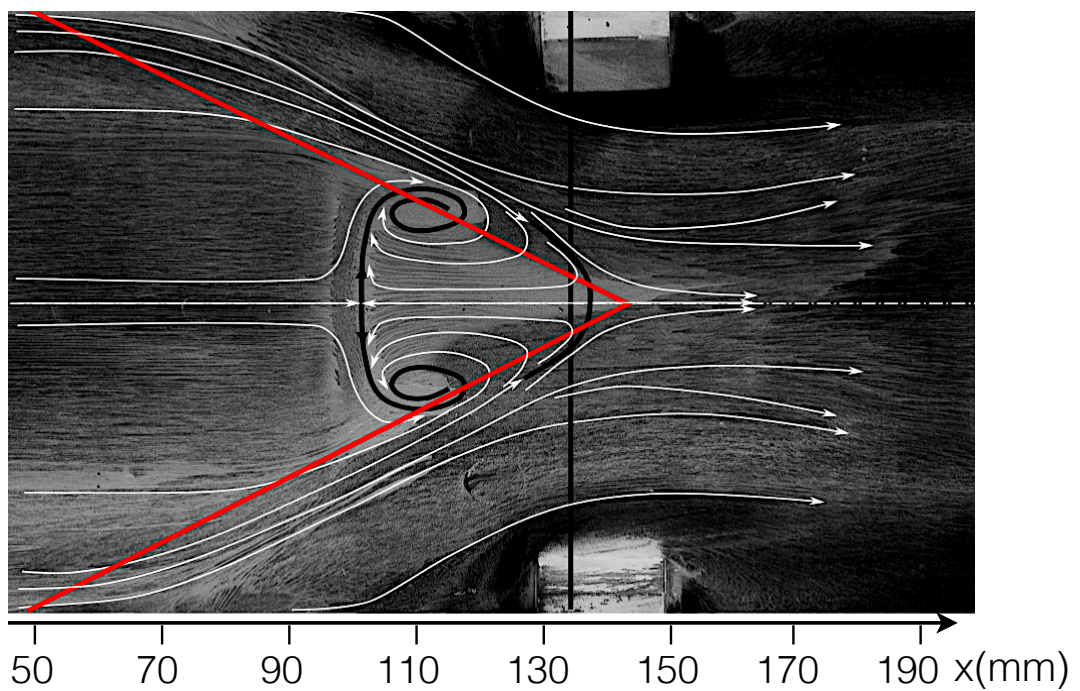

(a)

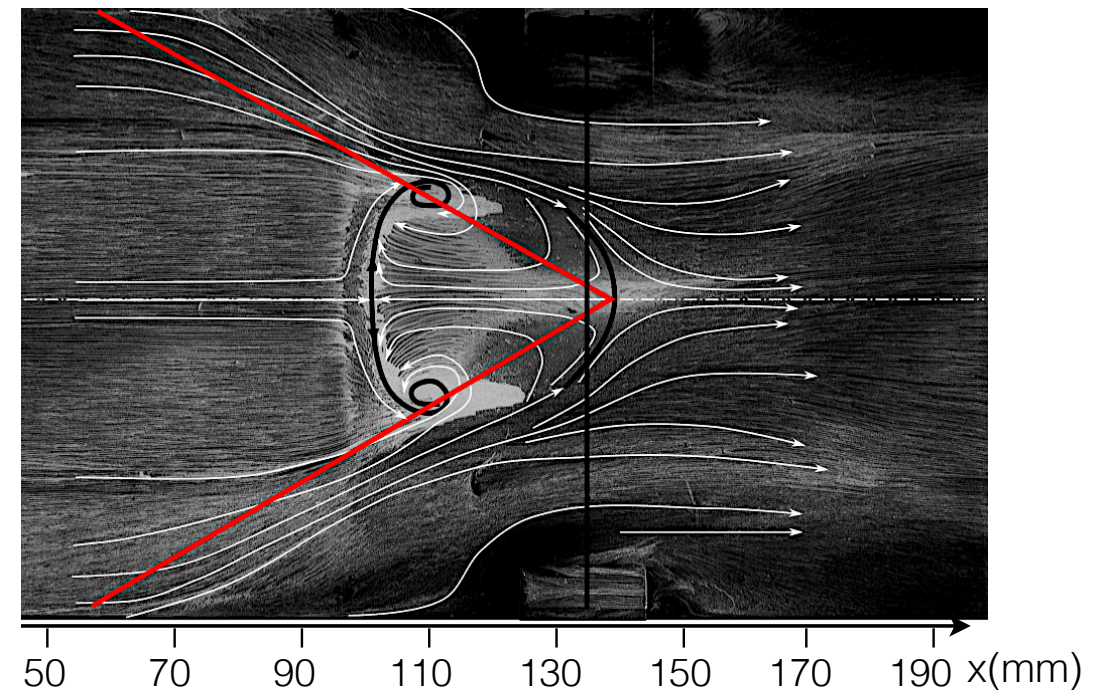

(b)

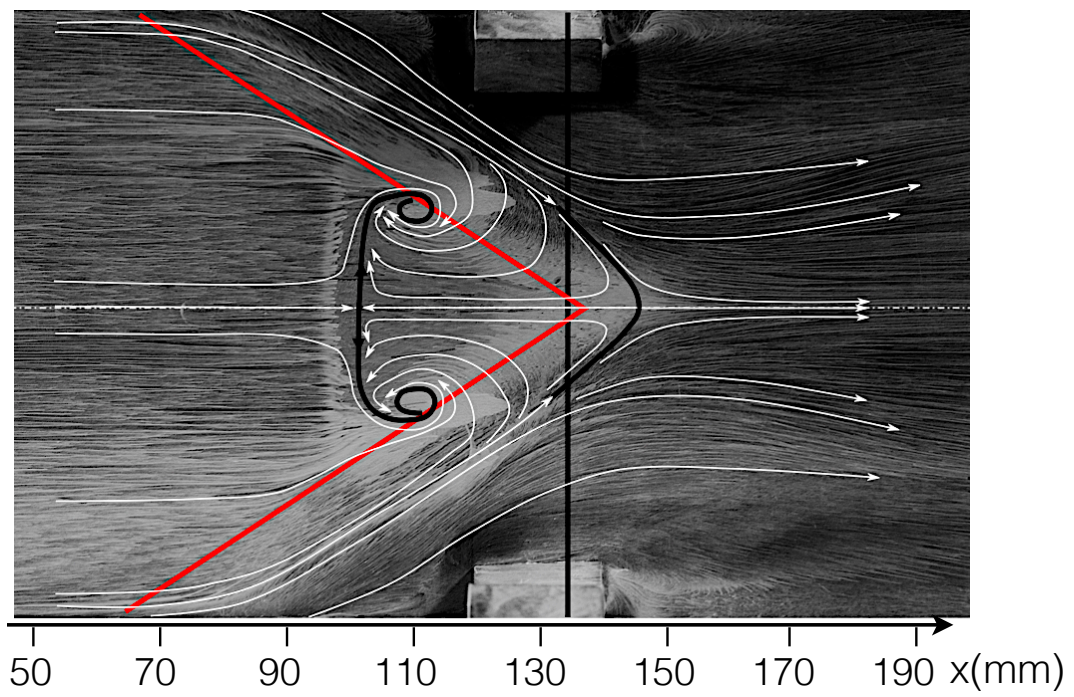

(c) 


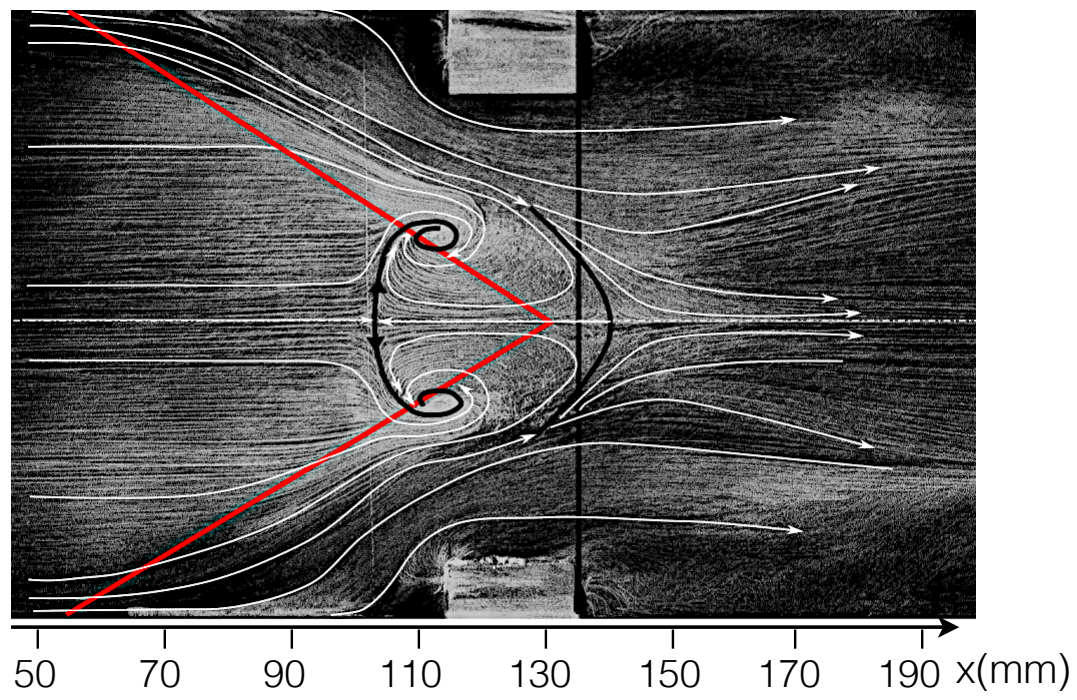

(d)

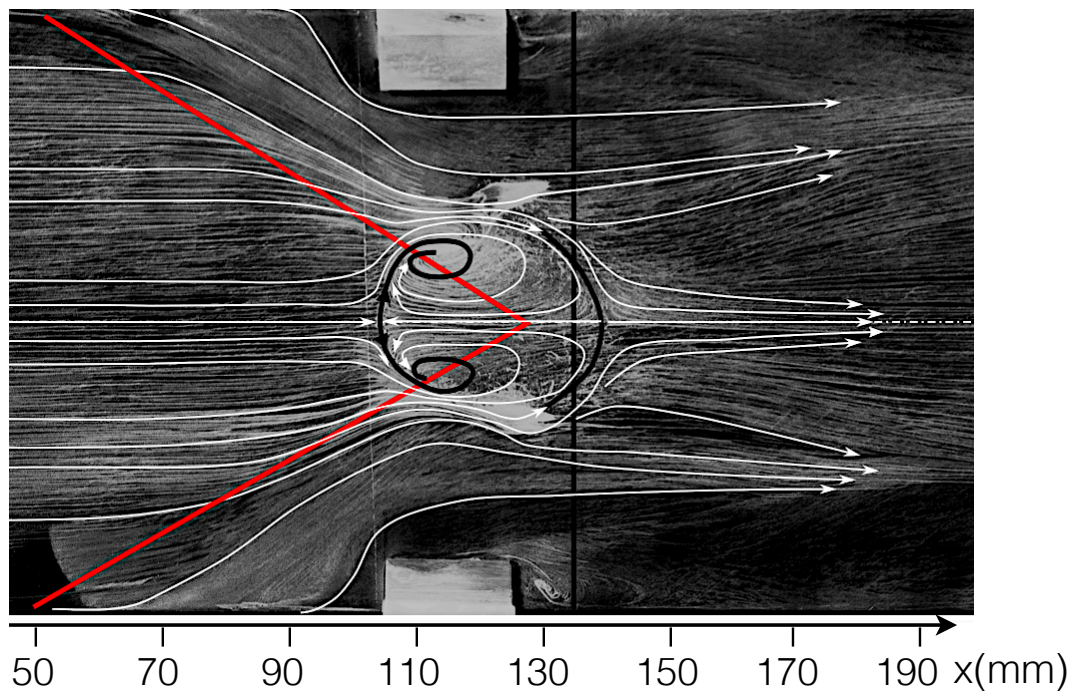

(e)

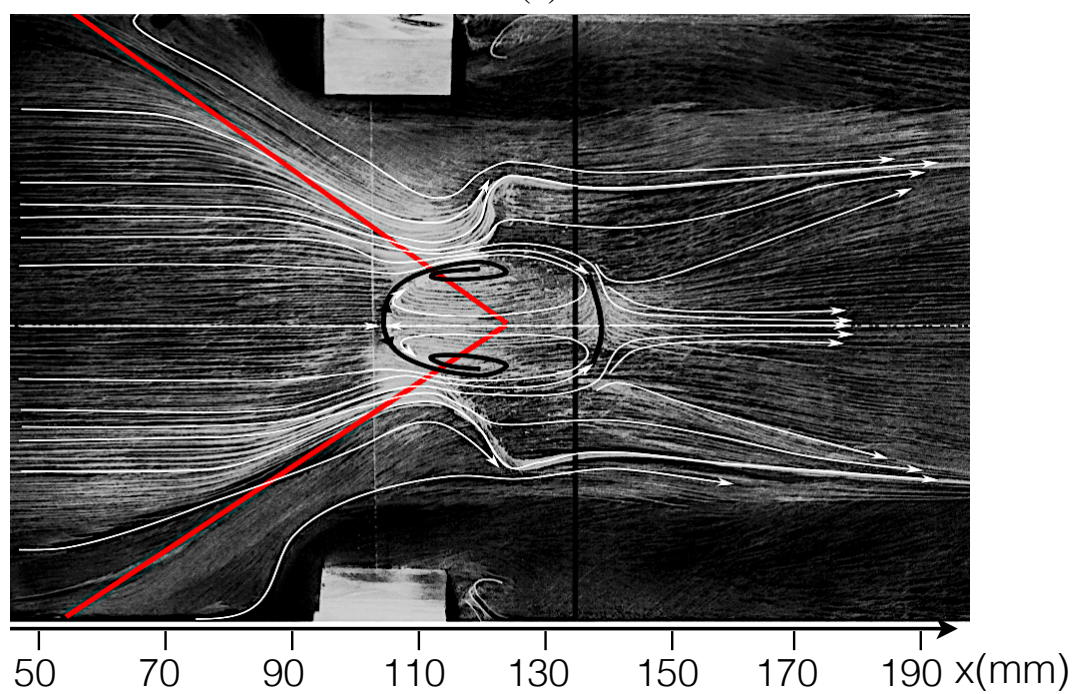

(f) 


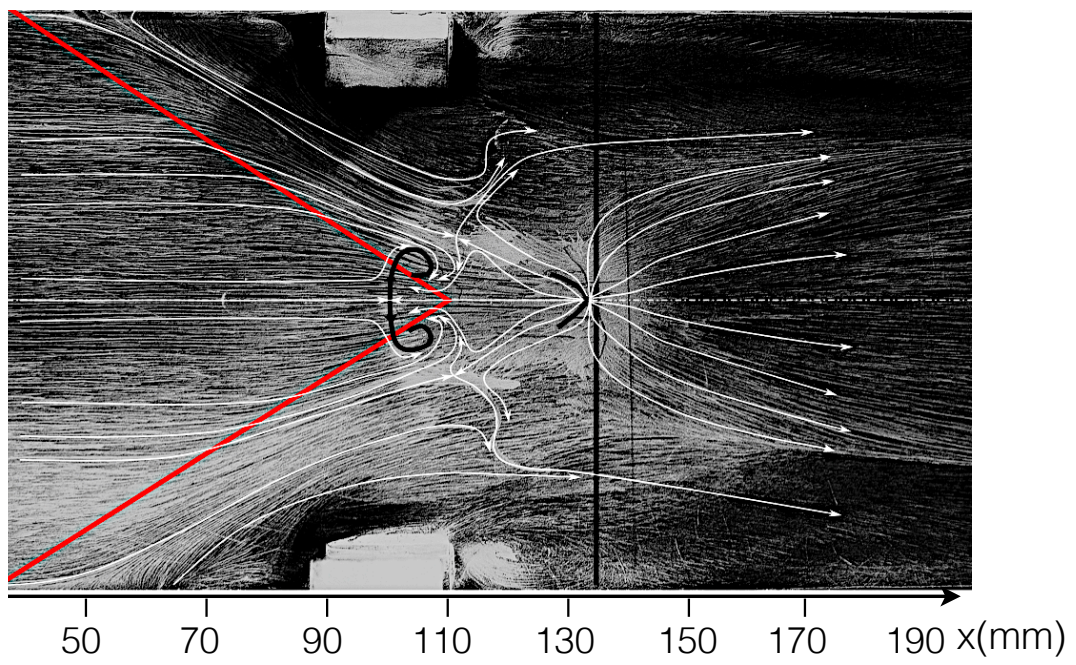

(g)

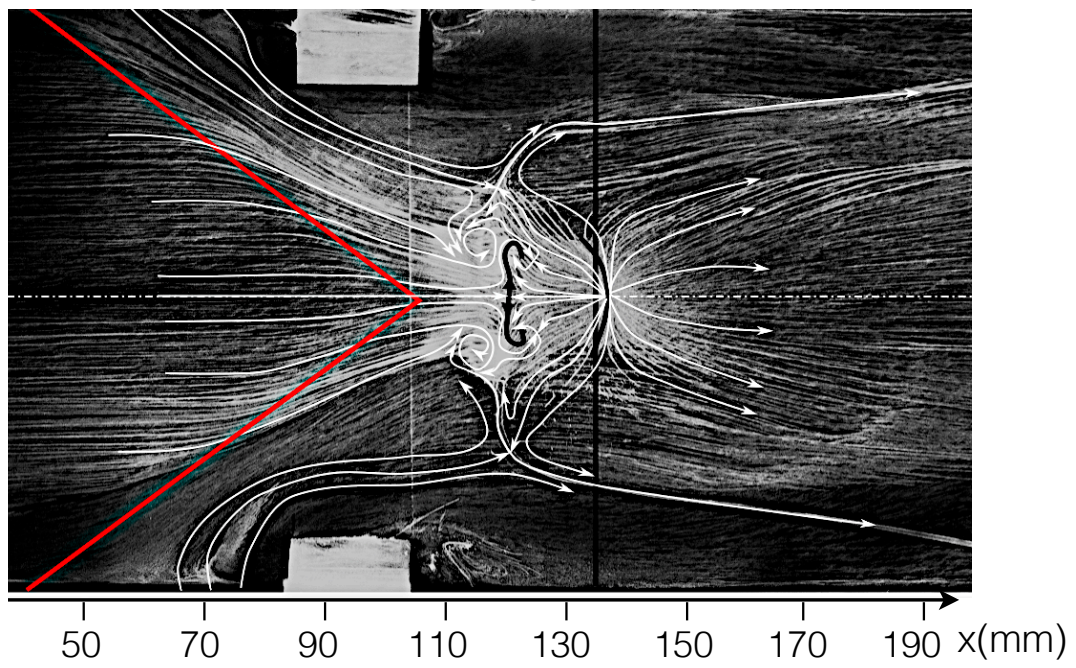

(h)

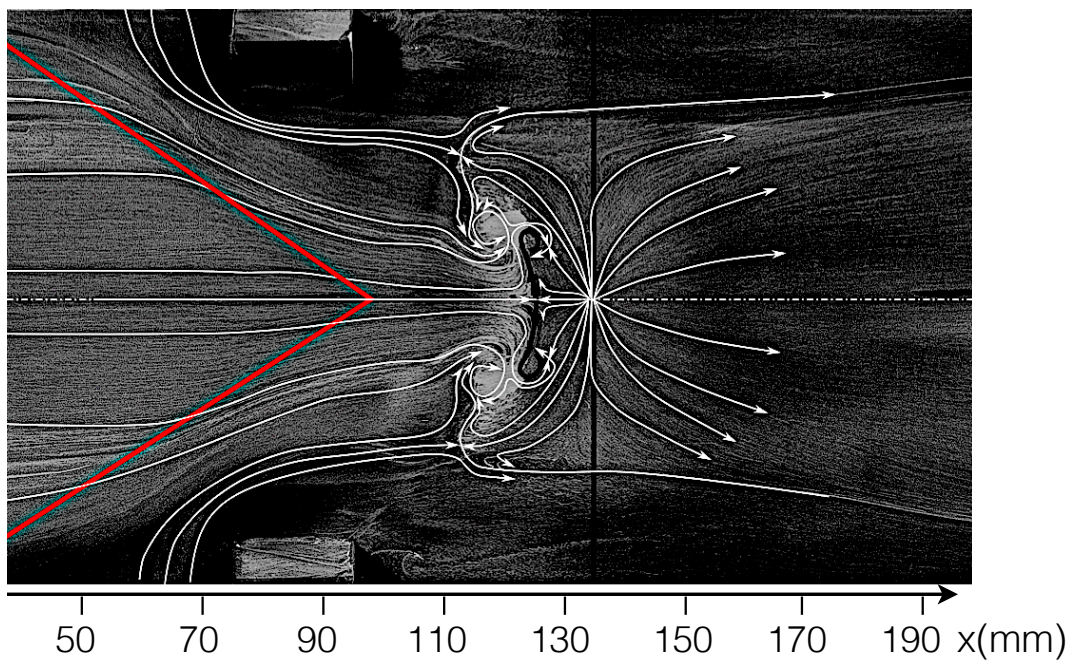

(i)

Fig. 4.27 Surface oil-flow visualisation on the tunnel floor for the oblique SBLIs with large corner blocks: $(a) x_{\text {block }}=130 \mathrm{~mm} ;(b) x_{\text {block }}=125 \mathrm{~mm} ;(c) x_{\text {block }}=120 \mathrm{~mm} ;(d) x_{\text {block }}=$ $115 \mathrm{~mm} ;(e) x_{\text {block }}=105 \mathrm{~mm} ;(f) x_{\text {block }}=95 \mathrm{~mm} ;(g) x_{\text {block }}=90 \mathrm{~mm} ;(h) x_{\text {block }}=85 \mathrm{~mm} ;(i)$ $x_{\text {block }}=75 \mathrm{~mm}$. The approximate footprint of the corner shock is indicated by red lines. 
(a)

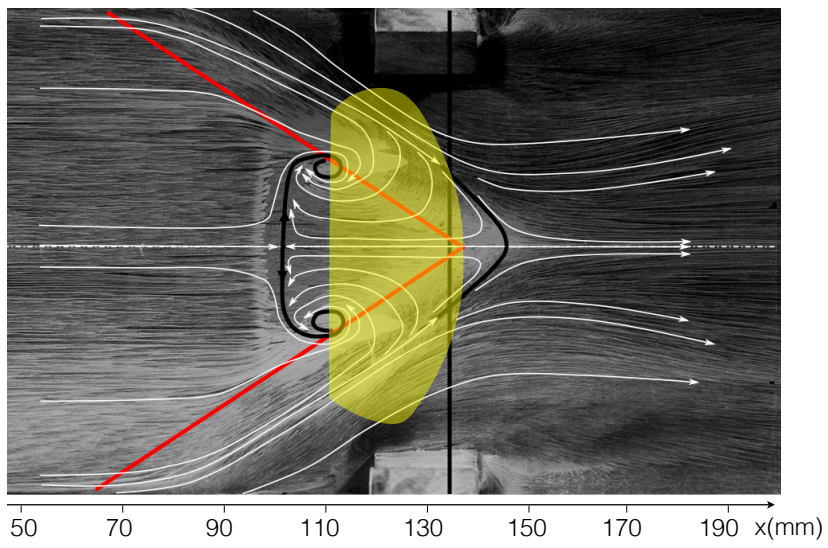

(b)

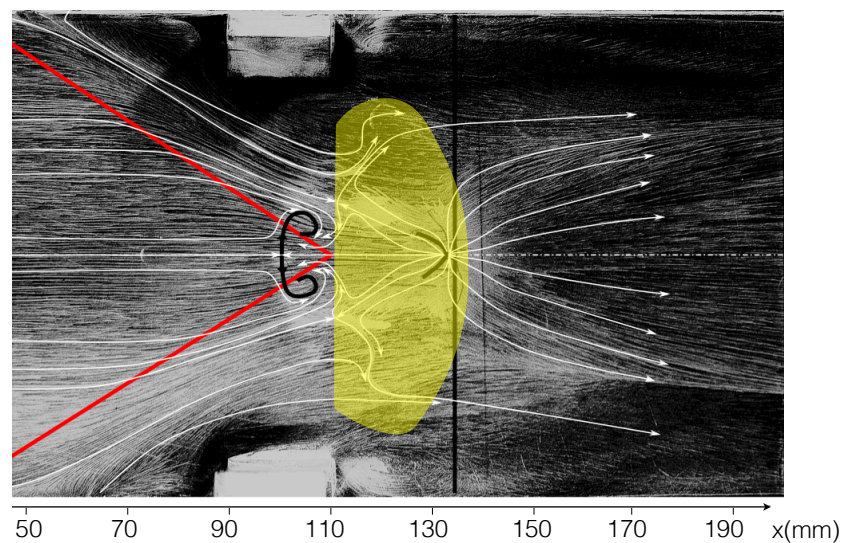

Fig. 4.28 Oil-flow visualisation on the tunnel floor with the central separation region of baseline interaction overlaid : (a) $x_{\text {block }}=120 \mathrm{~mm}$; (b) $x_{\text {block }}=90 \mathrm{~mm}$.

point moves upstream. As presented in figure $4.28 \mathrm{~b}$, for the case of $x_{\text {block }}=90 \mathrm{~mm}$, the crossing point is seen to fall at $x=112 \mathrm{~mm}$, where the central separation line of the undisturbed flow locates.

Due to the more prominent displacement effect caused by enlarged corner separations, corner shocks are located increasingly closer to the mid-span symmetry line and hence affect a larger portion of flow field. This is reflected by a change in the spanwise extent of the central separation, which clearly shows a trend of decreasing as the corner shocks move upstream.

The central separation topologies for the configurations discussed so far are presented and annotated schematically in figure 4.29. The flow topologies for the cases from $x_{\text {block }}=$ $130 \mathrm{~mm}$ to $x_{\text {block }}=95 \mathrm{~mm}$, as depicted in figure 4.29 a, can still be classified as 'owl-face of the first kind', characterised by a pair of saddle points and foci. However, when the corner shocks arrive ahead of the baseline interaction zone, the separation pattern is observed to transit to the more complicated 'owl-face of the second kind' (figure 4.29b). 
(a)

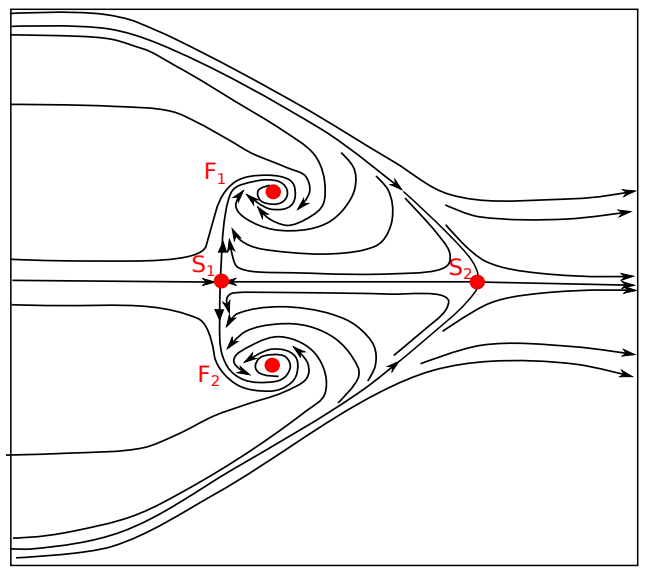

(b)

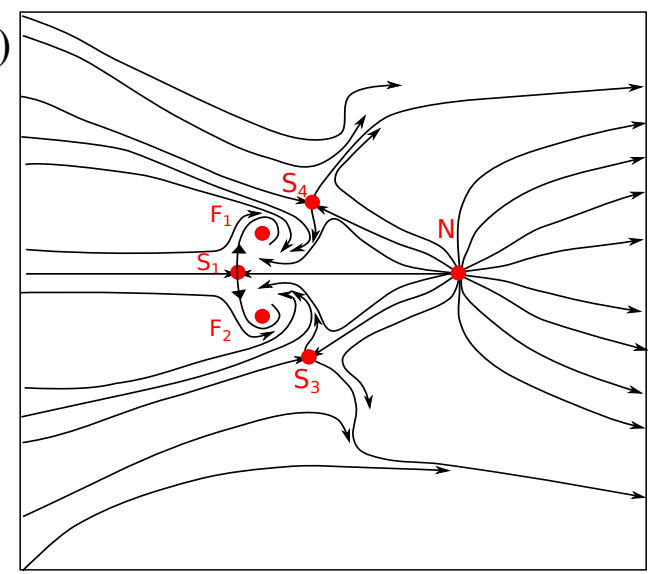

Fig. 4.29 Surface flow topology of the centreline separation underneath the oblique SBLIs when the crossing point of corner shocks locates inside the separated region: $(a)$ crossing point downstream to the separation onset of baseline flow, from $x_{\text {block }}=130 \mathrm{~mm}$ to $x_{\text {block }}=95 \mathrm{~mm}$; (b) crossing point at the separation onset of baseline flow, for $x_{\text {block }}=90 \mathrm{~mm}$.

As the crossing point of corner shocks shifts ahead of the separated zone (figures 4.27h,i), the trend of reducing separation length continues, even beyond the baseline flow. For the most upstream location of the corner blocks, the centreline separation length reduces to $9 \mathrm{~mm}$, which is a $64 \%$ decrease from the baseline value. As shown in figure 4.30 , the reduction in $L_{s e p}$ is due to a delayed separation onset, while the reattachment location remains approximately unchanged from the undisturbed interaction.

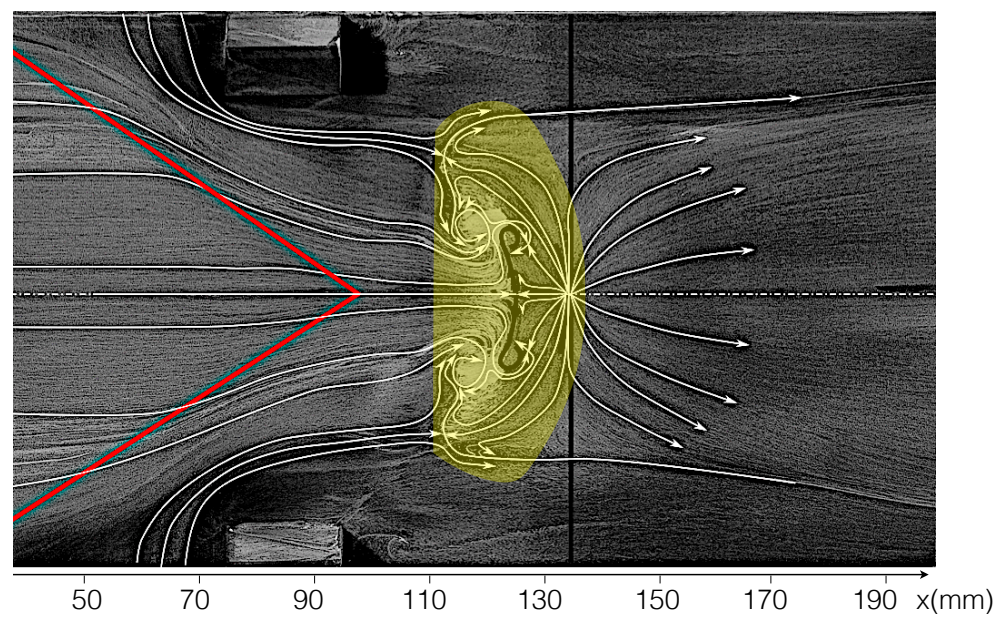

Fig. 4.30 Oil-flow visualisation on the tunnel floor for $x_{\text {block }}=75 \mathrm{~mm}$ with the central separation region of baseline interaction overlaid.

As presented in figure 4.31, the topological structure of the central separation is more complex for this flow case. Here an additional pair of saddle points $\left(S_{1}, S_{2}\right)$ and foci $\left(F_{1}, F_{2}\right)$ 


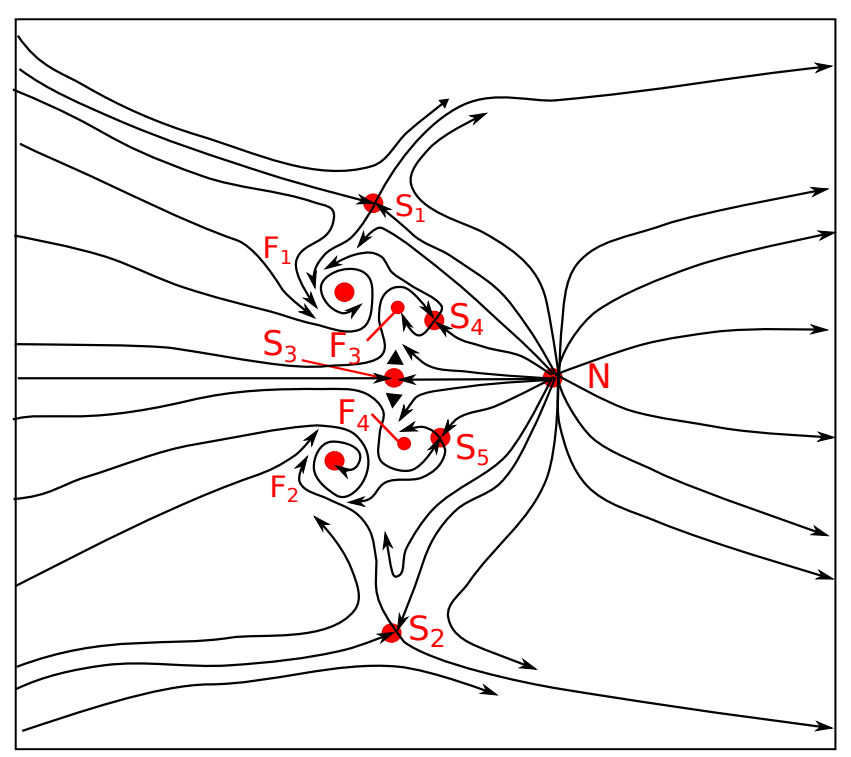

Fig. 4.31 Surface flow topology of the centreline separation underneath the oblique SBLIs when the crossing point of corner shocks locates ahead of the separated region, for $x_{\text {block }}=$ $85 \mathrm{~mm}$ and $x_{\text {block }}=75 \mathrm{~mm}$.

respectively are observed in the upstream portion, while the rest of the flow field closely resembles an 'owl-face of the second kind'. The more extensive corner separations deflect the flow near the sidewalls toward the mid-span more strongly. This, along with a reduced centreline separation distance, gives rise to an additional pair of vortices $\left(F_{1}, F_{2}\right)$, which act to help the flow accumulated in the central region lift off and escape from the tunnel floor. In this case, the spanwise width $L_{f}$ is defined as the distance between $F_{3}$ and $F_{4}$, the two focal points inside the shock-induced separated region.

Figure 4.32 shows the PSP map on the tunnel floor for the experiment with the largest $L_{\text {sep }}$, where the corner blocks are employed at $x_{\text {block }}=120 \mathrm{~mm}$. Compared to the flow with small blocks applied (figure 4.23), an even larger amount of pressure smearing is observed in the corners. The pressure rise through the main interaction in the central region also appears more gradual.

In figure 4.33 the static wall pressure distribution on the tunnel floor is shown for the two corner-block experiments with reduced $L_{\text {sep }}$. The corner shock footprint covers a larger area ahead of the main interaction in comparison with the baseline experiment. As a result of the more upstream position of corner shocks, there is no longer a uniform region directly ahead of the interaction domain.

Figure 4.34 compares selected wall pressure distributions along the tunnel floor centreline. The $x_{\text {block }}=85 \mathrm{~mm}$ case shows an increased upstream influence, with the pressure rise occurring approximately $12 \mathrm{~mm}$ earlier than in the baseline flow. This is followed by a less 


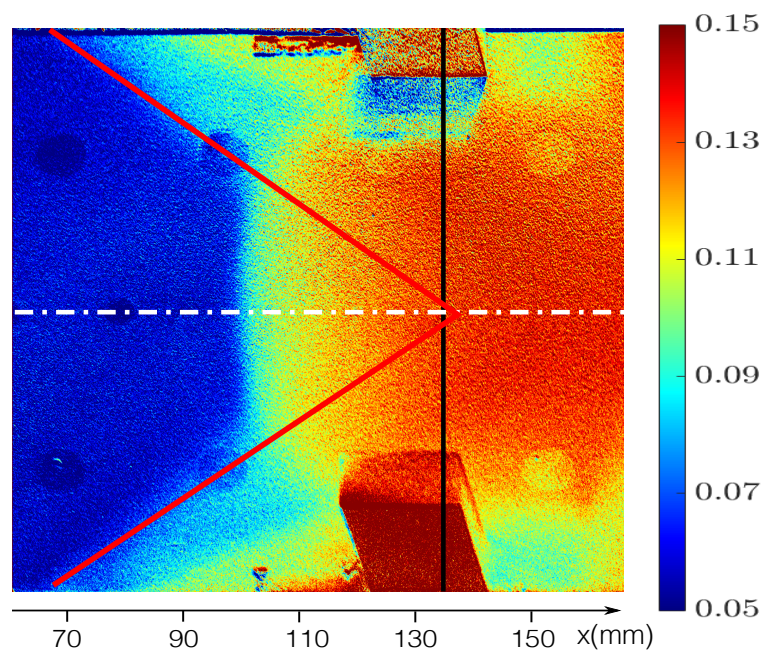

Fig. 4.32 $P / P_{0}$ for the tunnel floor for $x_{\text {block }}=120 \mathrm{~mm}$ with increased $L_{\text {sep }}$.

(a)

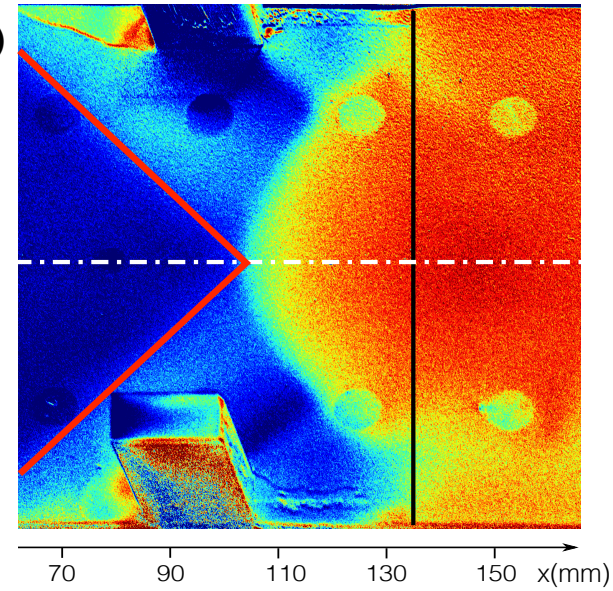

(b)

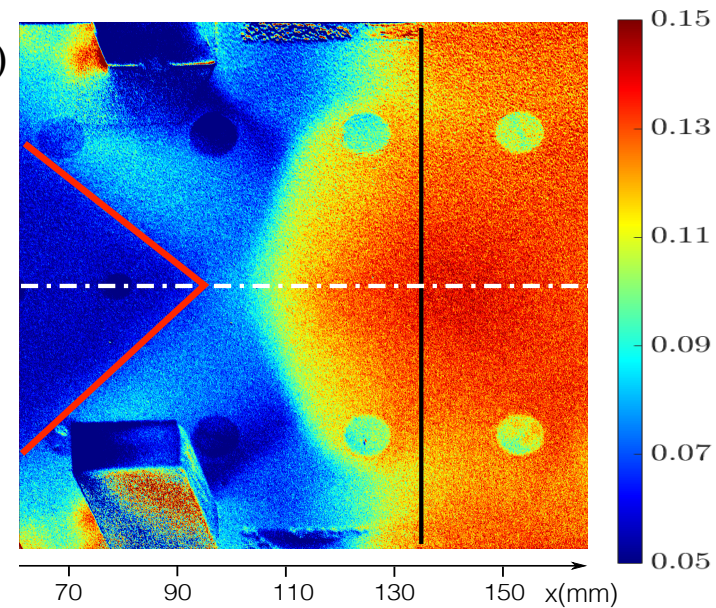

Fig. $4.33 P / P_{0}$ for the tunnel floor for the oblique SBLIs with reduced $L_{\text {sep }}$ : $(a) x_{\text {block }}=$ $85 \mathrm{~mm}$; $(b) x_{\text {block }}=75 \mathrm{~mm}$. The approximate footprint of the corner shock is indicated by red lines.

steep APG across the interaction and an unchanged total shock-induced pressure rise. For the $x_{\text {block }}=75 \mathrm{~mm}$ experiment, the upstream influence is further increased with the onset of pressure rise located $20 \mathrm{~mm}$ upstream compared to the baseline, exhibiting a larger interaction length with a noticeably milder adverse pressure gradient. If the centre-span flow behaved quasi-two-dimensionally, the larger interaction length would be associated with an increase in centreline separation. This, however, contradicts the considerably reduced $L_{s e p}$ shown in the oil-flow images (figures $4.27 \mathrm{~h}, \mathrm{i}$ ), suggesting that the increase in pressure smearing is not caused by the local separation 'bubble' but rather a consequence of the changes to the corner wave positions and their influence on the centreline flow. 


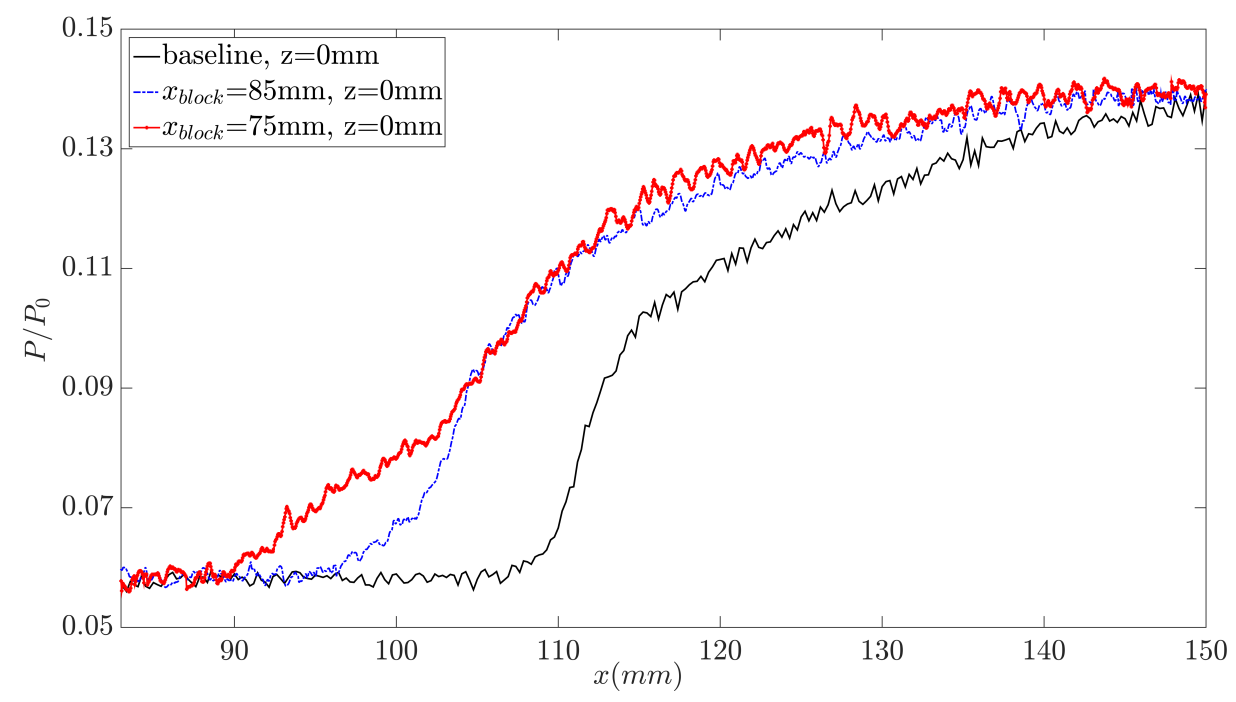

Fig. 4.34 Static wall pressure distribution on the tunnel floor centreline.

The inflow boundary layer velocity profiles along the tunnel floor centreline are presented in figure 4.35, with the corresponding parameters listed in table 4.6. The data for the configuration $x_{\text {block }}=75 \mathrm{~mm}$ is not available as this block position obstructs the laser light path. In all cases, the inflow profiles are almost unchanged from the baseline. This confirms that the corner obstacles, located well away from the tunnel mid-span, do not affect the centre-span flow ahead of the interaction.

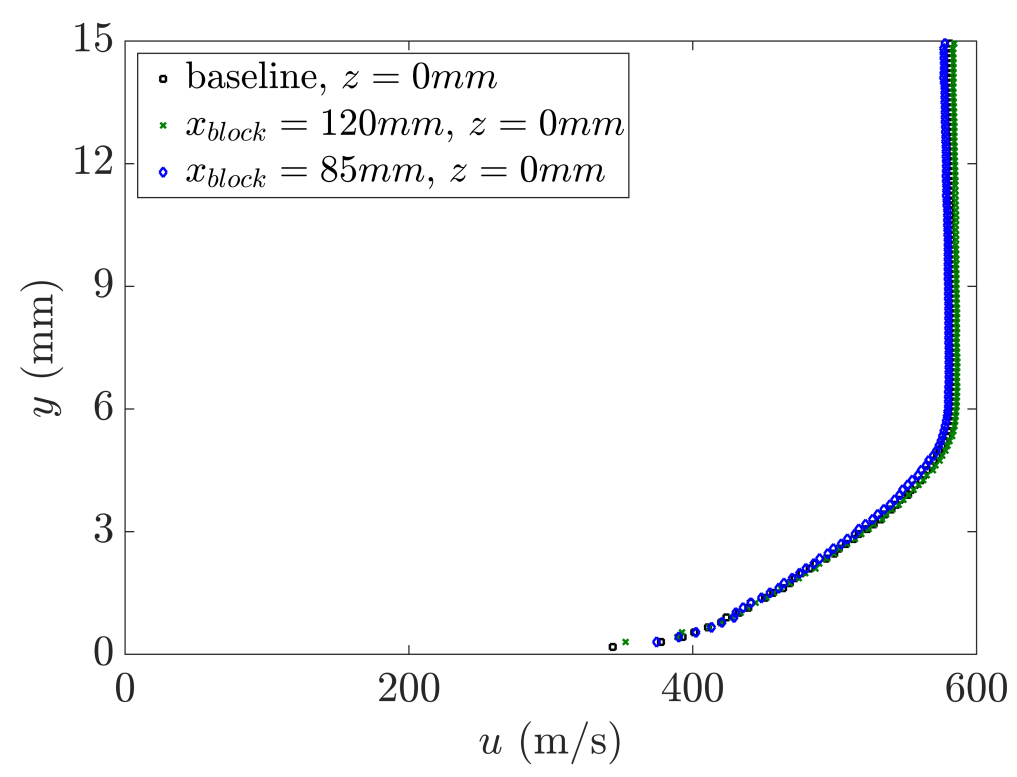

Fig. 4.35 Boundary layer velocity distribution at $x=80 \mathrm{~mm}$ for the tunnel floor centreline. 
Table 4.6 Boundary layer parameters at $x=80 \mathrm{~mm}$ along the tunnel floor centreline for experiments with increased corner separations.

\begin{tabular}{lcccc}
\hline Experiment & $\delta(\mathrm{mm})$ & $\delta^{*}(\mathrm{~mm})$ & $\theta(\mathrm{mm})$ & $\mathrm{H}$ \\
\hline$x_{\text {block }}=120 \mathrm{~mm}$ & 6.1 & 0.84 & 0.61 & 1.37 \\
$x_{\text {block }}=85 \mathrm{~mm}$ & 6.2 & 0.84 & 0.61 & 1.38 \\
\hline
\end{tabular}

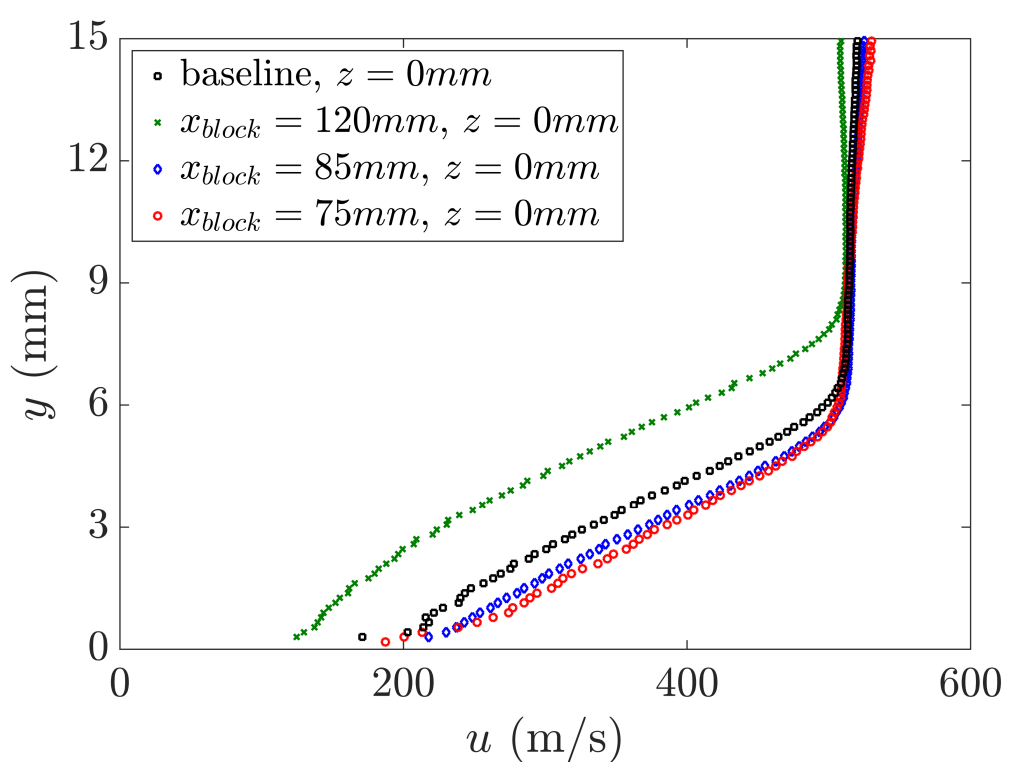

Fig. 4.36 Boundary layer velocity distribution at $x=160 \mathrm{~mm}$ for the tunnel floor centreline.

Table 4.7 Boundary layer parameters at $x=160 \mathrm{~mm}$ along the tunnel floor centreline for experiments with increased corner separations.

\begin{tabular}{lcccc}
\hline Experiment & $\delta(\mathrm{mm})$ & $\delta^{*}(\mathrm{~mm})$ & $\theta(\mathrm{mm})$ & $\mathrm{H}$ \\
\hline$x_{\text {block }}=120 \mathrm{~mm}$ & 10.4 & 3.10 & 1.61 & 1.93 \\
$x_{\text {block }}=85 \mathrm{~mm}$ & 7.0 & 1.77 & 1.04 & 1.71 \\
$x_{\text {block }}=75 \mathrm{~mm}$ & 6.8 & 1.66 & 1.00 & 1.67 \\
\hline
\end{tabular}

Figure 4.36 compares the boundary layer profiles measured $25 \mathrm{~mm}$ downstream of the inviscid shock reflection point. A less full profile is seen in the $x_{\text {block }}=120 \mathrm{~mm}$ experiment, which is in agreement with the higher $\mathrm{H}$ in table 4.7. Here the boundary layer is also much thicker, as observed in the schlieren visualisation in figure 4.25a. This is in accordance with the delayed reattachment process and the largest separation length $L_{s e p}$ among all the tests. Lower incompressible shape factors are observed for the experiments with the corner blocks applied at $x_{\text {block }}=85 \mathrm{~mm}$ and $x_{\text {block }}=75 \mathrm{~mm}$, which correspond to the fuller boundary layer 
profiles recovering quicker from the effect of the oblique interaction. This is most likely a consequence of the reduced centreline separation.

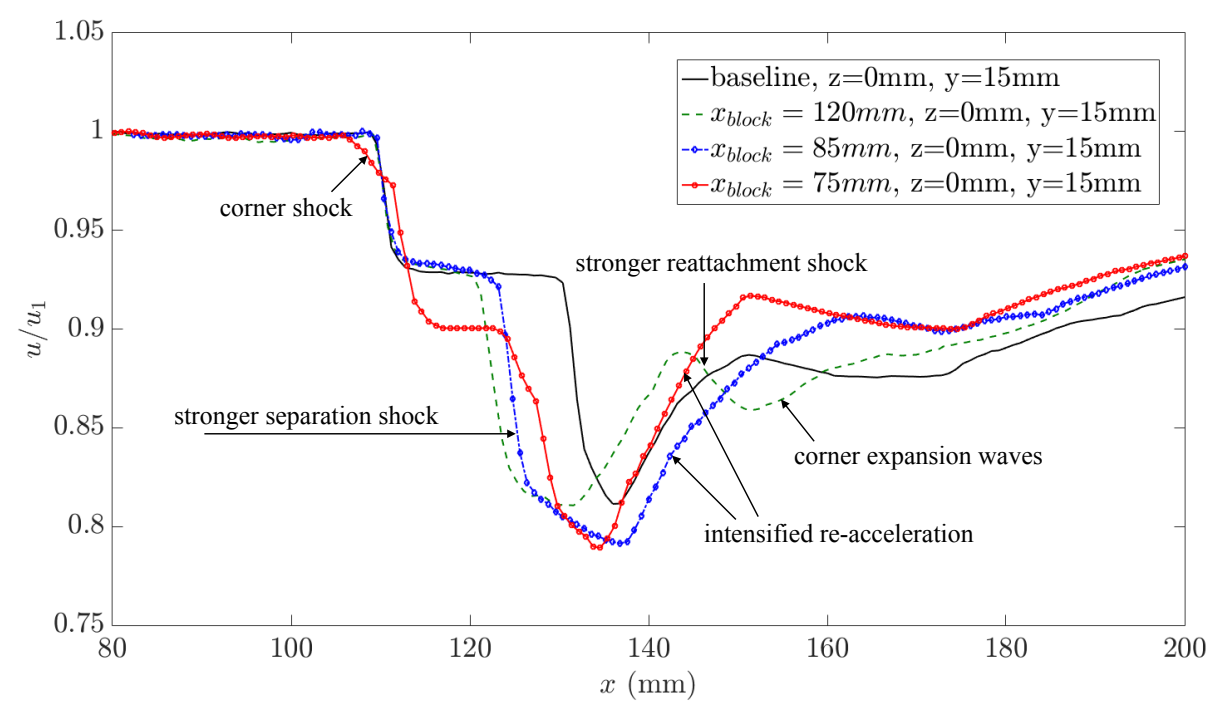

Fig. 4.37 Variation in streamwise velocity at $y=15 \mathrm{~mm}, z=0 \mathrm{~mm}$ for flows with increased corner separations.

Figure 4.37 presents the streamwise velocity variation through the impinging SBLI above the boundary layer edge at $y=15 \mathrm{~mm}, z=0 \mathrm{~mm}$. The measurements show that the deceleration through the separation shock occurs approximately $10 \mathrm{~mm}$ further upstream in the three large-corner-block tests than in the baseline experiment, in line with an increased interaction length and an earlier onset of pressure rise. For the $x_{\text {block }}=120 \mathrm{~mm}$ test the flow deceleration through the reattachment shock is more pronounced, indicating that the strength of the pressure rise is intensified by the corner shock waves which reach the symmetry line at this location (see also the approximate footprint of the corner shocks marked in figure $4.27 \mathrm{c}$ ). The expansion waves generated by the corner separation can be seen in the flow acceleration downstream of the reattachment shock. In the $x_{\text {block }}=85 \mathrm{~mm}$ experiment where corner shocks arrive ahead of the centreline separation, the flow is decelerated to a lower velocity than the baseline case through the combined effects of the corner and separation shocks. This is followed by a more pronounced re-acceleration caused by the subsequent corner expansion waves, which also meet inside the interaction zone. For the case of $x_{\text {block }}=75 \mathrm{~mm}$, an additional velocity reduction occurs ahead of the incident shock. This is due to the corner shocks being shifted further upstream, now located approximately $30 \mathrm{~mm}$ ( 5 inflow $\delta$ ) ahead of the centreline separation onset, as shown in figure $4.27 \mathrm{i}$. The following deceleration of the incident shock appears to be almost the same as for the baseline case. A similar intensified re-acceleration is again observed. 

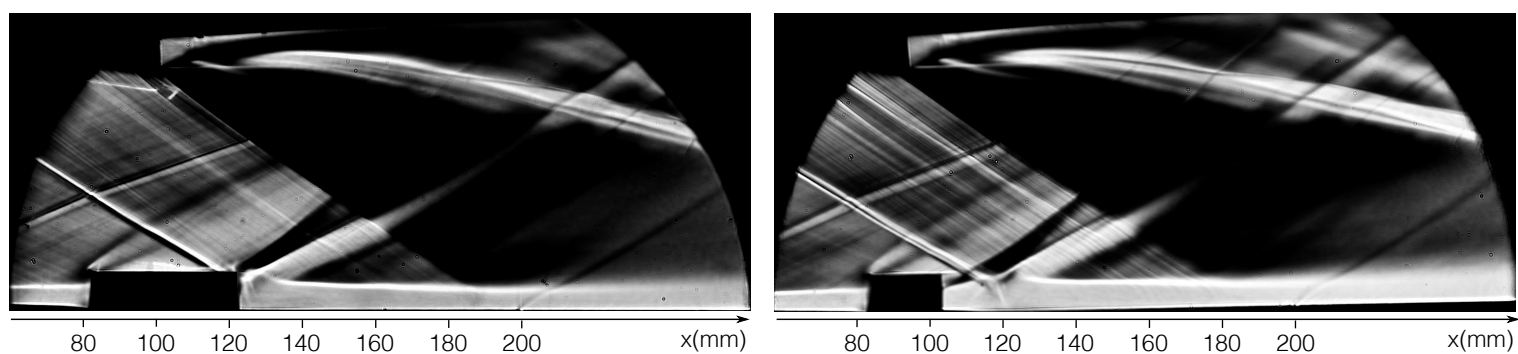

Fig. 4.38 Schlieren photographs with large corner obstacle applied at $x_{\text {block }}=85 \mathrm{~mm}:(a)$ elongated blocks of $L_{\text {block }}=40 \mathrm{~mm}$; $(b)$ standard blocks of $L_{\text {block }}=20 \mathrm{~mm}$.

To test whether the prominent corner expansion waves measured by the velocity variation are induced by the corner separation or the rear edge of the block, an additional experiment has been conducted with obstacles of a different length of $L_{\text {block }}=40 \mathrm{~mm}$. The schlieren visualisation in figure 4.38 shows that the wave patterns of the oblique SBLIs are very similar for both flow fields.

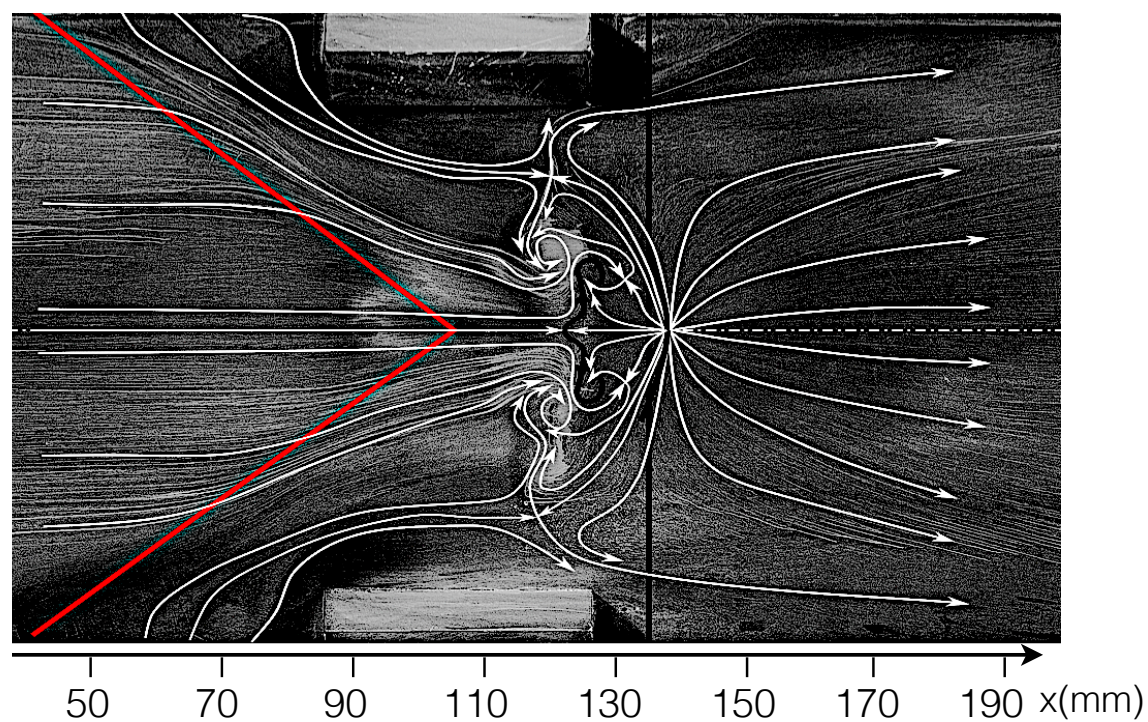

Fig. 4.39 Surface oil-flow visualisation on the tunnel floor with $L_{\text {block }}=40 \mathrm{~mm}$ blocks applied at $x_{\text {block }}=85 \mathrm{~mm}$.

Figure 4.39 displays the oil-flow image on the tunnel floor. It is seen that $L_{s e p}$ of the flow disturbed by the elongated obstacles is $15 \mathrm{~mm}$, almost the same as that altered by the standard blocks (figure $4.27 \mathrm{~h}$ ). This suggests that the expansion waves emanating from the tunnel sidewall are a genuine feature of the convex shape of the corner separation.

In figure 4.40 the boundary layer profiles of these two experiments measured along the tunnel floor centreline are compared, with the data of inflow shown in figure 4.40a and flow downstream of the interaction in figure 4.40b. It is observed that the velocity profiles are very 


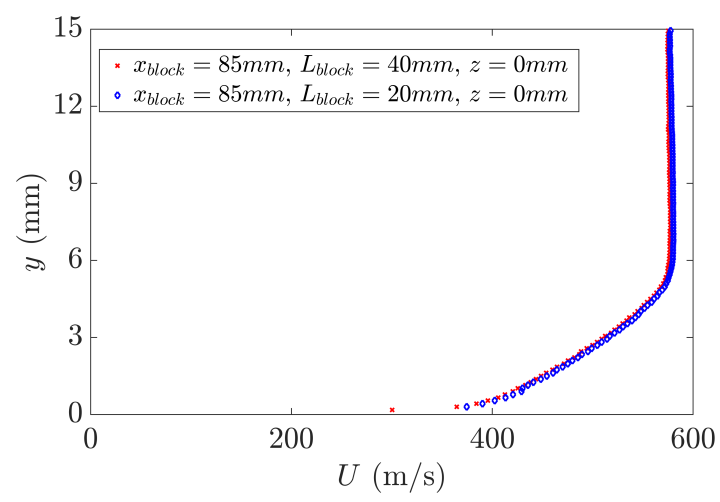

(a)

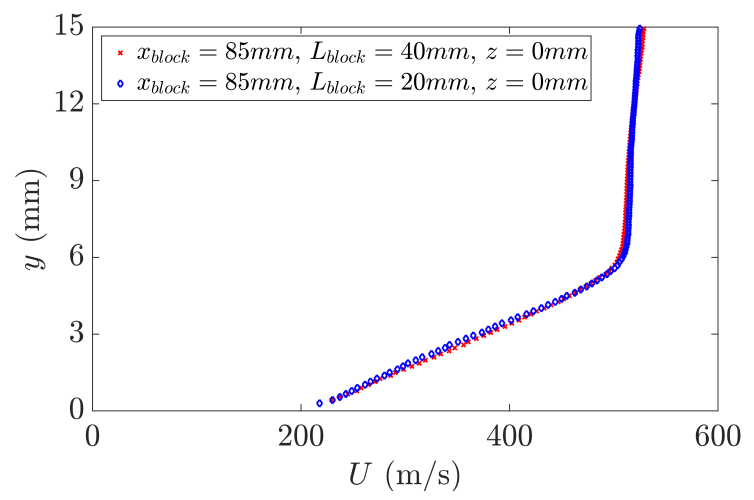

(b)

Fig. 4.40 Boundary layer velocity distribution for different block lengths $L_{\text {block }}$ along the tunnel floor centreline: $(a)$ at $x=80 \mathrm{~mm} ;(b)$ at $x=160 \mathrm{~mm}$.

similar, confirming that different block lengths did not affect the location and influence of the corner waves in the central region.

\subsection{Effects of corner separation on flow field}

\subsubsection{Flow behaviour}

In the above it has been shown that modifications to the geometry of corner separations can strongly effect the size and shape of separation observed in the centre of the tunnel floor. A decrease in the size of corner separation generates a more two-dimensional flow field with the central separation having a greater spanwise extent (table 4.2). An increase in the size of the corner separation drastically changes the surface flow topology of the separated region in the centre, which is observed to transit from the quasi-two-dimensional 'owl-face of the first kind' to the more complicated second kind (shown in figures 4.29 and 4.31). The separation length measured along the symmetry line is also seen to be considerably altered, varying from $36 \%$ to $180 \%$ of the nominally two-dimensional baseline value (refer to table 4.5 ).

The measurements also provide information on the three-dimensional wave pattern induced by sidewall effects. The blockage effect of the corner separation first deflects the supersonic flow away from the corner through a series of compression waves, and then returns the flow towards the streamwise direction by an expansion fan (illustrated in figures 4.9, 4.10a). In the tunnel floor PSP maps and oil-flow images it has been observed that the changes to the size of corner separation have a direct effect on the location of these corner waves. 


\subsubsection{The factor influencing corner effects}

Analysis of the results from flow visualisations reveals that there is an interesting linking pattern between the location of corner waves and the central separation geometry. More specifically, the position of the corner 'shock' crossing point, found by approximating the corner compression waves by a straight line, is shown to give a good indication of the impact of corner effects on the main interaction. If the crossing point falls downstream of the interaction the separation on the centreline is unaffected. If the crossing point falls inside the interaction zone the separation is increased, while a crossing point ahead of the interaction leads to a reduction in centreline separation length.

This behaviour is well illustrated by figure 4.41 which shows the dimensionless separation length measured on the symmetry line, defined as the ratio of $L_{s e p}$ to the incoming boundarylayer displacement thickness $\delta^{*}$, as a function of the corner shock crossing point for all experiments conducted. As a guide, the extent of the baseline separation is also given. For corner shock crossing points well downstream of the interaction there is no influence on the separation length and the central separation is at its most 'two-dimensional'. As the crossing point approaches the separated region, an increase in $L_{\text {sep }}$ is observed, with the largest separation seen where the corner shocks arrive near the end of the baseline interaction. When the crossing point moves upstream inside the central separation, the separation length experiences a progressive decrease. In cases where the corner shocks are shifted further upstream and intersect ahead of the interaction, the reduction in $L_{s e p}$ continues even beyond the nominal two-dimensional value. The change in streamwise separation length due to corner effects is significant, with the largest separation being five times greater than the smallest.

The spanwise extent of the central separation $L_{f}$ is made dimensionless by dividing the values by the wind tunnel working section width $w$. As shown in figure $4.42, L_{f} / w$ presents a general trend of decreasing as the crossing point shifts upstream until it moves outside the separated region, where the central separation expands wider in spanwise direction with the corner shocks intersection point located further upstream. 


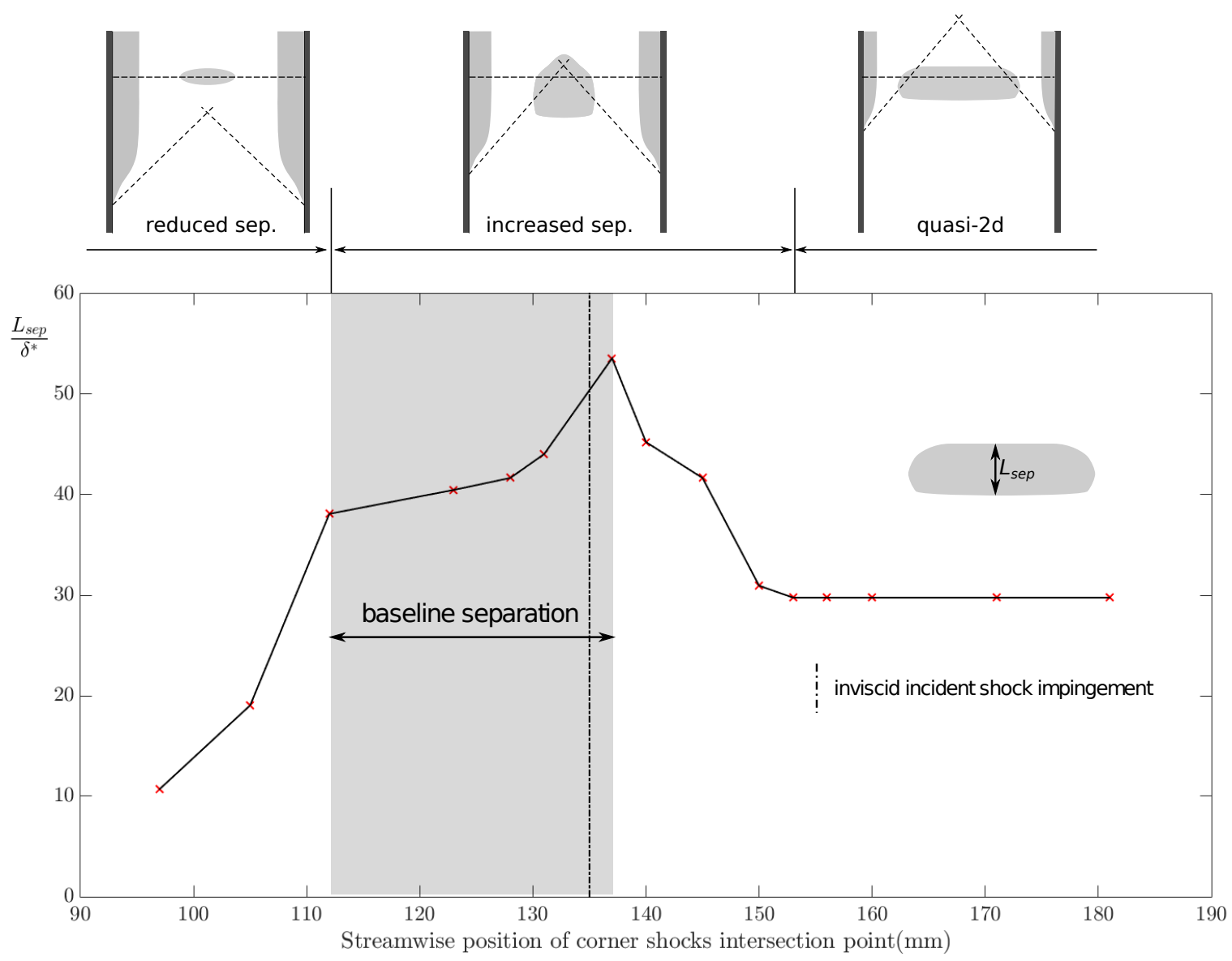

Fig. 4.41 Variation of the scaled centreline separation length $L_{s e p} / \delta^{*}$ as a function of streamwise position of corner shocks intersection point. 


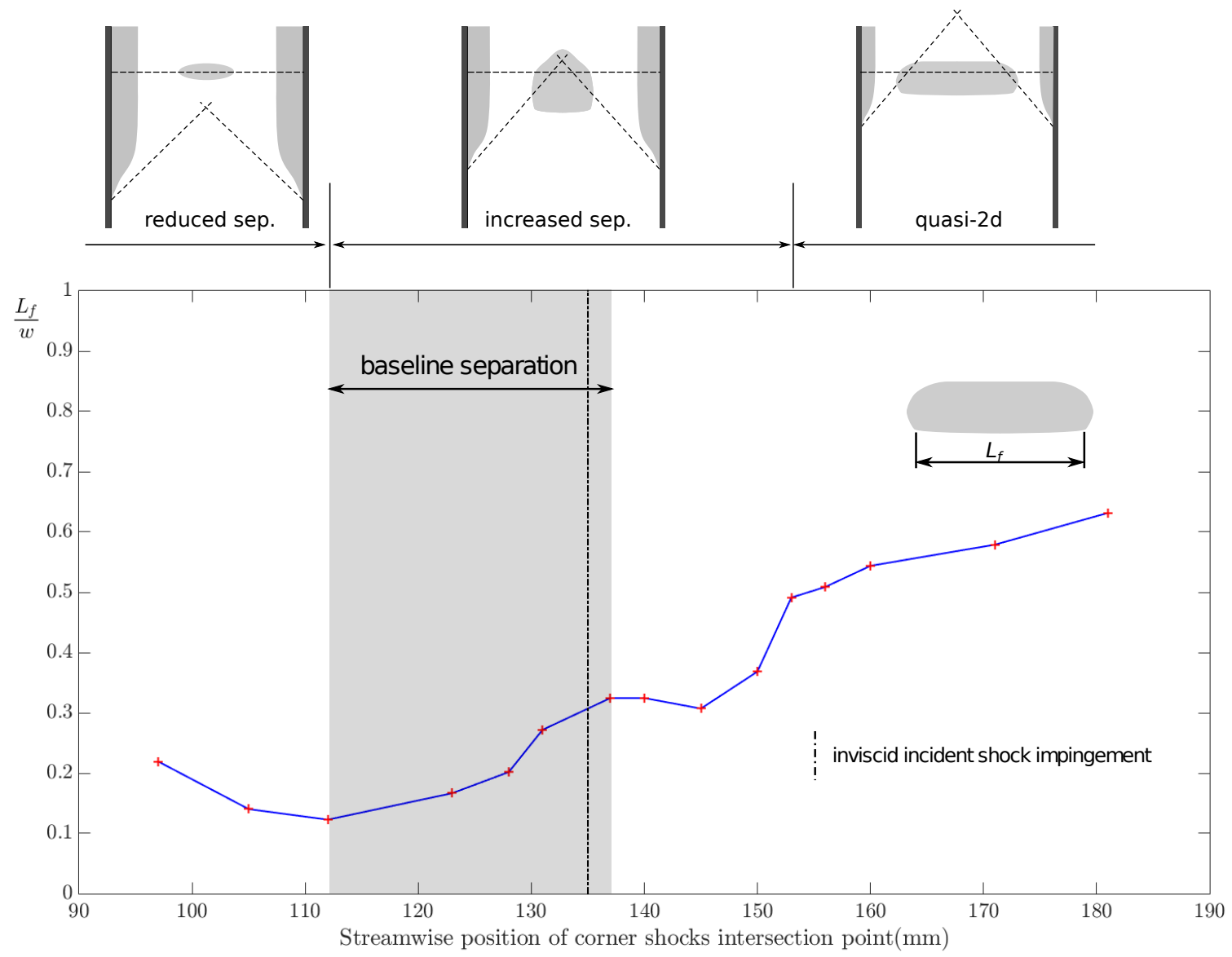

Fig. 4.42 Variation of the scaled centreline separation width $L_{f} / w$ as a function of streamwise position of corner shocks intersection point. 


\section{Chapter 5}

\section{Physical mechanism of corner effects}

\subsection{Influence of corner waves}

Examination of the experimental results shows that changes in the central separation underneath the main interaction are correlated with the position of the corner shock crossing point. The static wall pressure measurements indicate that whenever the size of corner separation is altered, the pressure smearing in the corner region changes accordingly. For the interactions with an affected centreline separation length, either increased (figure 4.32) or decreased (figure 4.33) in comparison with the nominally two-dimensional value, the adverse pressure gradient in the central region is also noticeably modified. In figure 4.34 selected wall pressure distributions along the mid-span symmetry line are compared, it has been observed that interactions with a reduced $L_{s e p}$ exhibit an increased upstream influence, featuring a milder adverse pressure gradient than the baseline case. This contradicts the expected behaviour of a 'quasi-two-dimensional' centre-span flow, where a larger interaction length would be associated with an increased separation length. This indicates that the changes observed in the mid-span are not caused by the local separation bubble, but rather a consequence of the corner effects.

The analysis suggests that the primary mechanism behind the coupling between the separations in corner and central regions is the generation of corner waves induced by the displacement effect of the corner separation. Both wall pressure (figure 4.9) and streamwise velocity (figure 4.37) measurements show that the corner waves consist of a series of compression waves followed by an expansion fan. For a supersonic interaction, these waves, which originate from the sidewalls and propagate towards the tunnel centre, can pass through the oblique reflection and thereby alter the interaction wave structure. The adverse pressure gradient imposed upon boundary layers elsewhere is in turn modified, leading to an increase or reduction in the local separation length. 


\subsection{Three-dimensional oblique SBLI structure}

To verify the proposed mechanism based on the influence of corner waves, flow visualisation and quantitative measurements are combined together to form diagrams of the likely oblique SBLI structures for the cases with unchanged, increased and reduced $L_{s e p}$ respectively.

\subsubsection{Corner effects on 'quasi-two-dimensional' interactions}

The effects of the corner waves on the nominal two-dimensional interactions are shown schematically in figure 5.1. Figure 5.1a defines three distinct regions (I-III) depending on the relative position of the corner waves and the main interaction domain.

For the actual region of corner flow (I), the boundary layer typically features considerable low-momentum fluid as a result of the viscous interactions with two surfaces (floor and sidewall). Therefore, although a weaker adverse pressure gradient is seen in the near-wall region, corner separation is generally observed to occur earlier than elsewhere. In the sketch here the onset of corner separation is hence upstream of the central separation line.

In the region between the corner separations and the centre (II), the oncoming flow first meets the corner waves, which produce an adverse and then favourable pressure gradient, before encountering the main interaction. As shown in figure $4.9 \mathrm{~b}$, the pressure downstream of the expansion fan is approximately the same as that of the inflow, indicating that the pressure rise induced by the corner shock is offset by the following expansion waves. A milder APG is hence produced due to a combination of an earlier onset of pressure rise caused by corner shock and an unchanged total shock-induced pressure rise as in the twodimensional oblique SBLI because of the cancellation effect of the subsequent expansion. This reduced APG, as evidence by the tunnel floor pressure distribution (figures 4.6, 4.12b, $4.19 \mathrm{~b}$ ), is the most likely cause of the reduction or even absence of separation.

The influence of the wave pattern on the flow in the central symmetry plane is shown in figure $5.1 \mathrm{~b}$, which is typical of the dominant features in region III. Here the corner shocks and the following expansion waves pass through the incident shock and its reflection, and arrive well behind the oblique interaction. The SBLI is therefore not influenced by corner effects, which is in agreement with the similar boundary layer profiles (tables 4.3, 4.4) and streamwise velocity variation (figure 4.20) measured at $z=0 \mathrm{~mm}$ for the baseline case and all the experiments with reduced corner separations. As a result, the separation topology in-between the corner shocks (i.e. the location of the upstream leading compression wave as explained in figure 4.10) exhibits a 'quasi-two-dimensional' pattern with relatively straight separation and reattachment lines, confirming that region III is outside the influence of corner effects. 
This explanation is confirmed by the interaction with corner separations slightly larger than the nominally-two-dimensional baseline flow as shown in figure 4.22. In such a scenario region II is noticeably wider as corner shocks locate closer to the centre-span and hence influence a larger portion of flow field (figure 4.23). Region III however characterizes with an almost unmodified separation length since the corner shock crossing point is still sufficiently downstream to leave the mid-span flow affected.

(a)

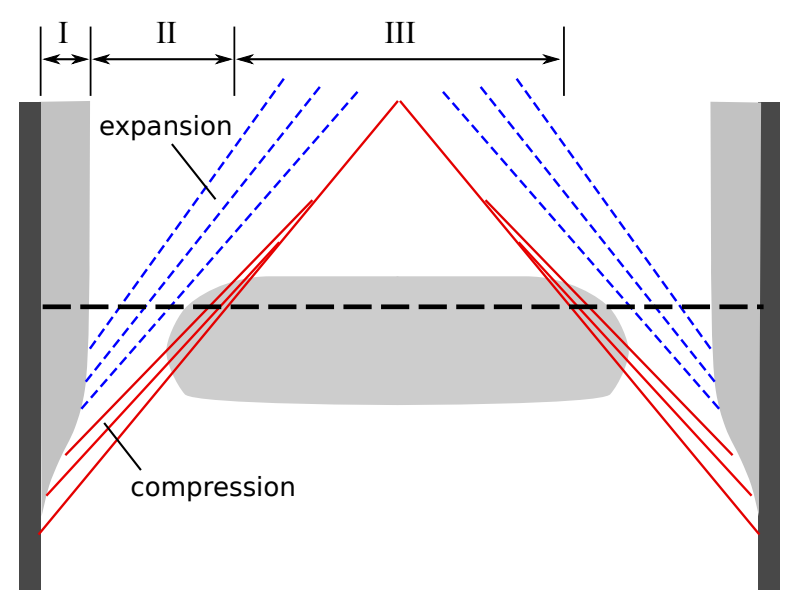

(b)

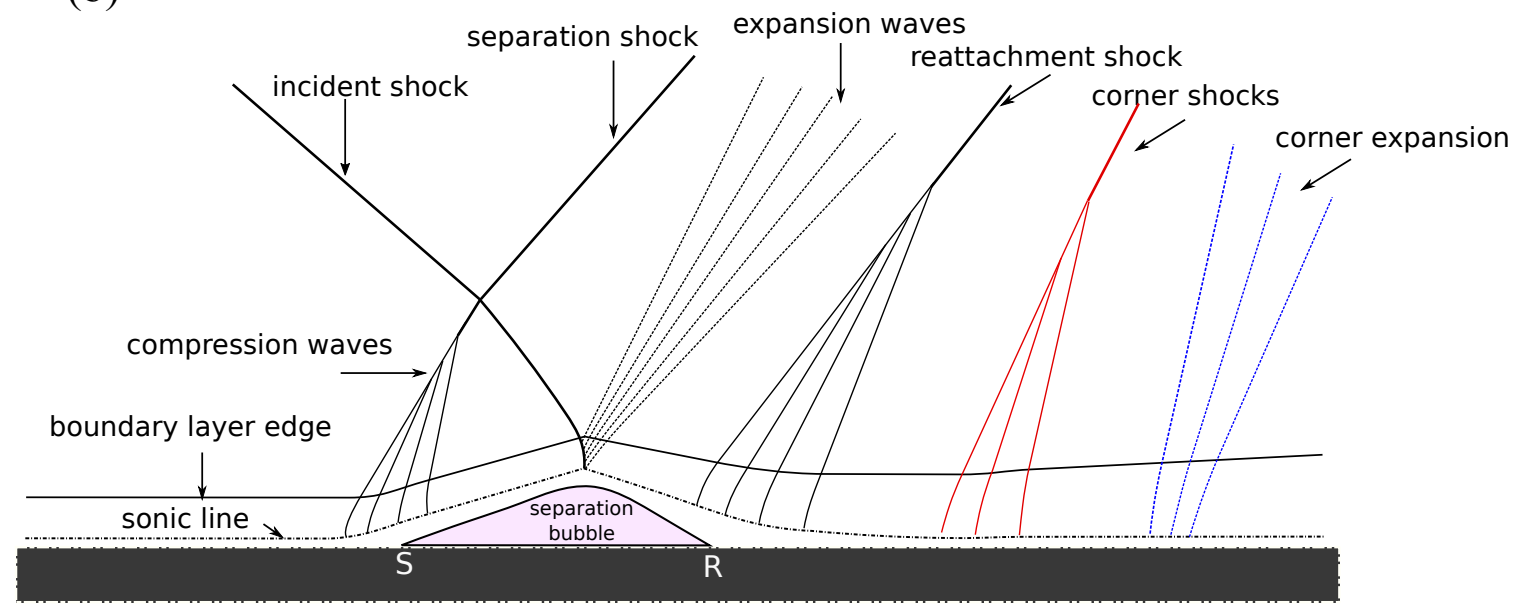

Fig. 5.1 Schematic illustration of corner effects on 'quasi-two-dimensional' interaction: $(a)$ separation zones on floor; $(b)$ diagram of SBLI structure on the central symmetry plane. 


\subsubsection{Corner effects on interactions with increased separation length}

Figure 5.2 describes interactions where the corner shock crosses around the reattachment region (e.g. $x_{\text {block }}=120 \mathrm{~mm}$ ). In this scenario, the corner separation is significantly increased in size (indicated in figure 5.2a by an enlarged region I) and deflects the near-wall flow towards the centre of the tunnel more strongly. The corner shocks hence are located increasingly closer to the mid-span of the tunnel and eventually move inside the separated region. Corner effects therefore influence a larger proportion of the flow field and the quasi-two-dimensional part of central separation reduces accordingly. Region II, characterized by additional pressure smearing through the SBLI, now widens, which is reflected by the trend of decreasing $L_{f}$ as the corner shocks shift forwards into the interaction zone (figure 4.42).

In the central region (IV) corner compression waves enter the interaction domain and an increase in the local separation length is seen. Following the diagram presented previously for quasi-two-dimensional interactions (figure 5.1b), the three-dimensional SBLI wave pattern in the central plane is provided in figure 5.2b. Here the reattachment shock is strengthened by the corner shocks. As the reattachment process is achieved through the energy-transfer by the shear layer at the edge of the separation bubble, the more severe APG imposed by the intensified reattachment shock would require an increased separation size to accommodate the greater overall pressure jump across the interaction. The subsequent corner expansion waves, which might relieve the additional pressure rise, however arrive too far downstream to alleviate the detrimental effect of the corner shocks before reattachment (as shown by the velocity measurement in figure 4.37). This leads to the increased separation length observed in the oil-flow visualisation (figure 4.27c). The enlarged centreline separation then gives rise to an increased interaction domain seen in the schlieren image (figure 4.25) and PSP map on the tunnel floor (figure 4.32), which explains the earlier onset of deceleration through the separation shock compared to the baseline case (figure 4.37).

The situation discussed above is the most unfavourable scenario as no beneficial expansion waves can reach the interaction, which explains why the largest separation length occurs when corner shocks approach the end of the separation (figure 4.41). As the corner shock crossing point shifts upstream from the reattachment location, more beneficial expansion waves can reach the interaction and help to gradually alleviate the detrimental effects of the corner shocks. This is in accordance with the progressive reduction in $L_{s e p}$ observed in oil-flow images presented by figures $4.27 \mathrm{~d}$-g. 
(a)

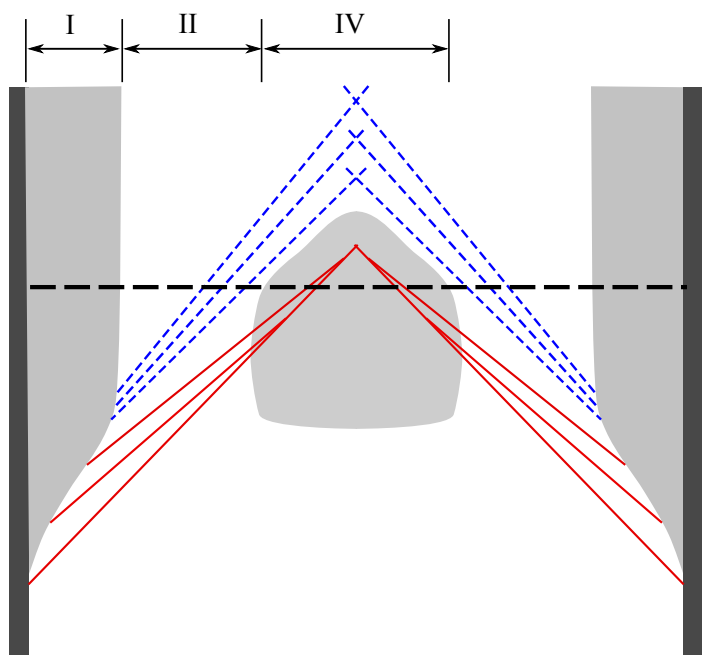

(b)

reattachment shock strengthened by corner shocks

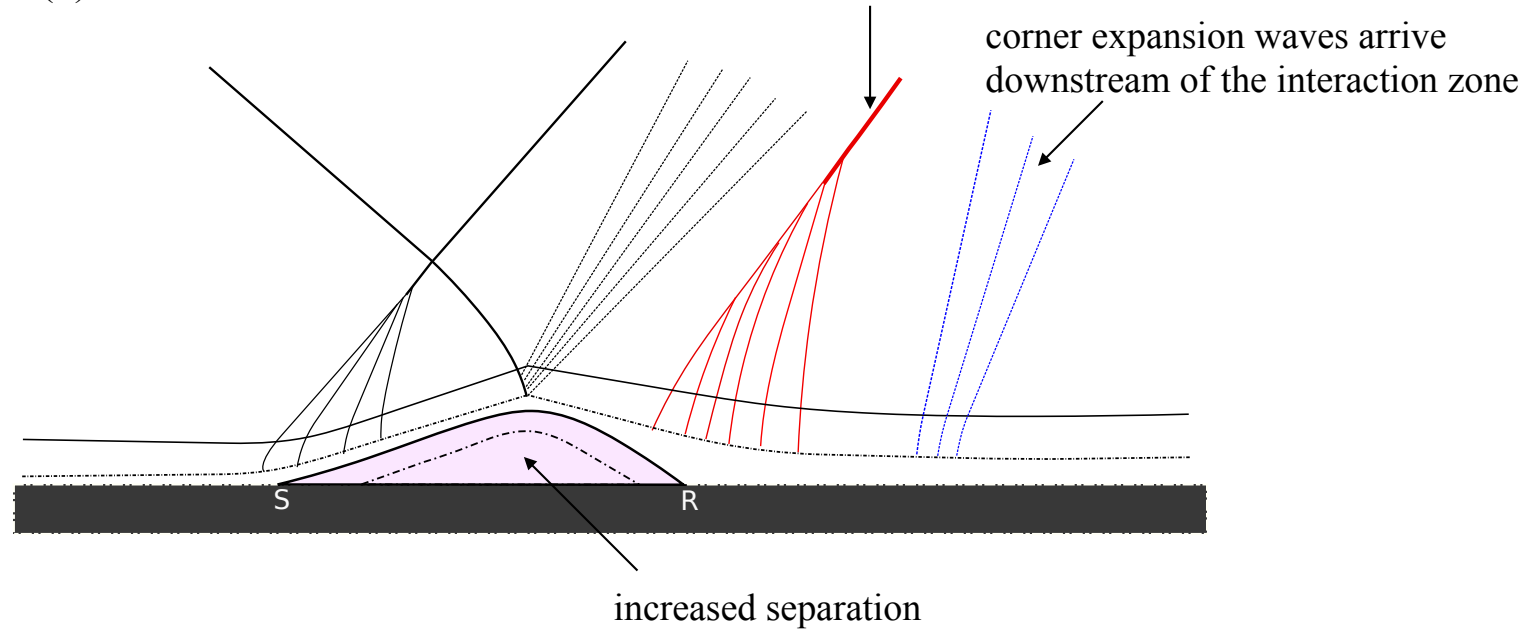

Fig. 5.2 Schematic illustration of corner effects on interactions with increased $L_{\text {sep }}$ : $(a)$ separation zones on floor; $(b)$ diagram of SBLI structure on the central symmetry plane. 


\subsubsection{Corner effects on interactions with reduced separation length}

A schematic illustration for the interaction where the corner shocks are even further upstream and cross ahead of the separated region is provided in figure 5.3. Here the corner shocks reach the centreline before the separation point (refer to region $\mathrm{V}$ in figure 5.3a). The additional pressure rise caused by the corner shock might be expected to increase the central separation length and move the separation onset upstream, whereas the evidence (figures 4.27h-i, 4.39)

(a)

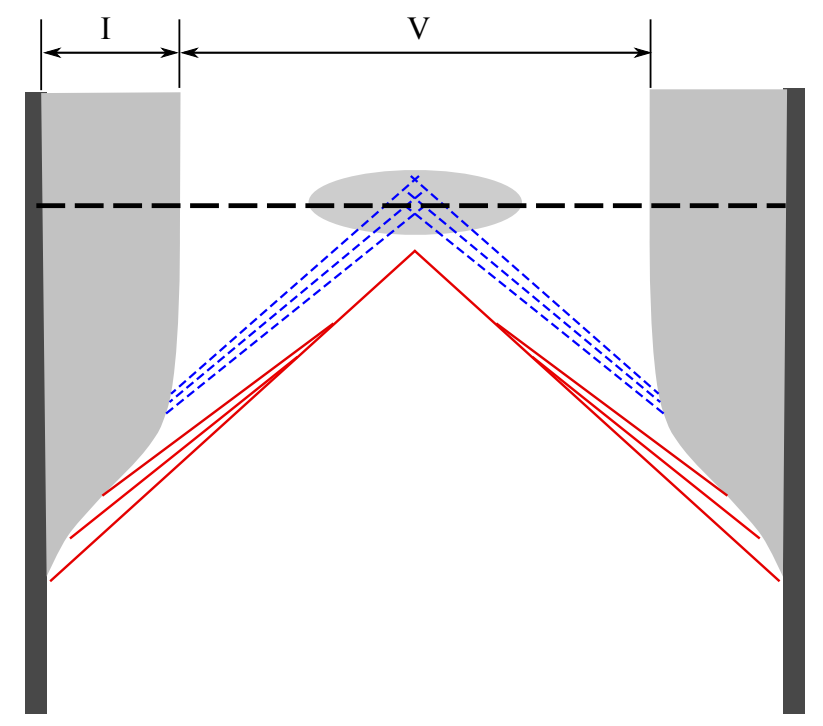

(b) separation shock strengthened by corner shocks

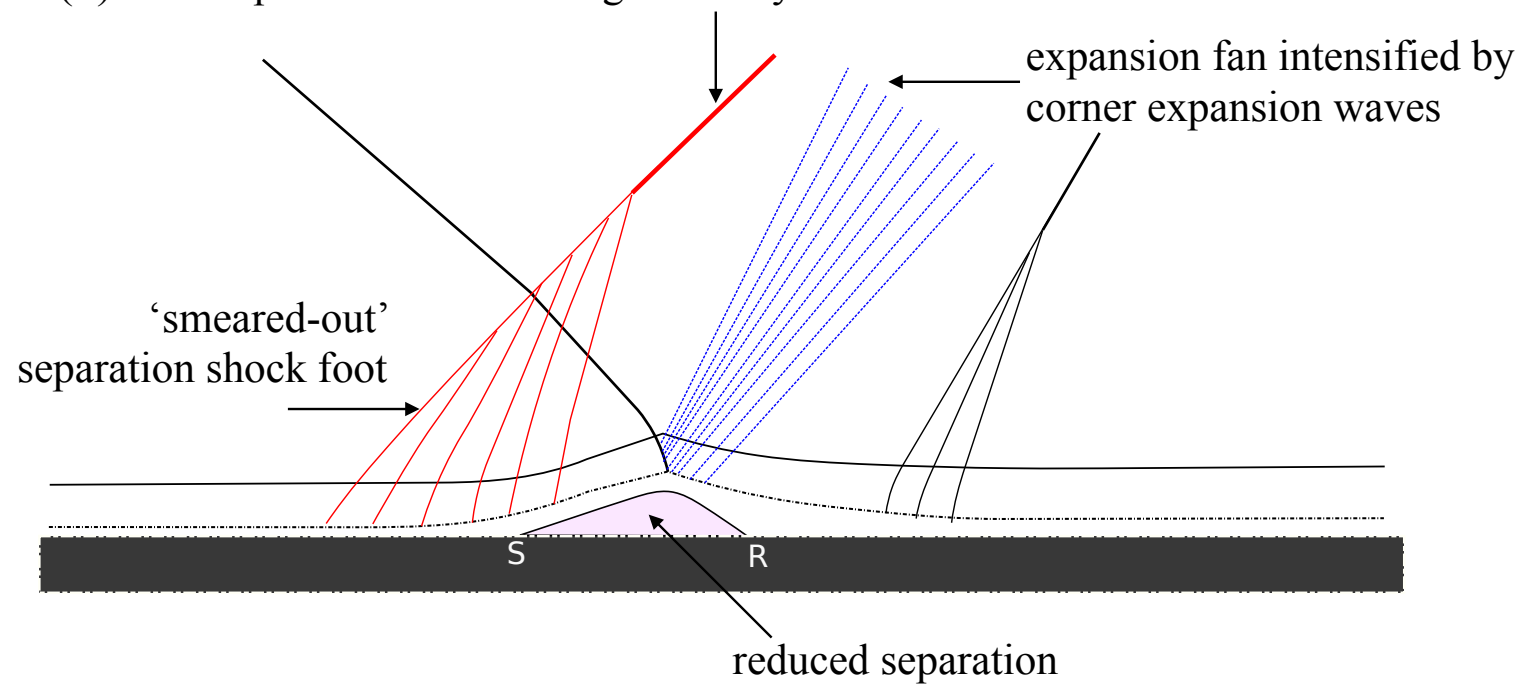

Fig. 5.3 Schematic illustration of corner effects on interactions with reduced $L_{\text {sep }}$ : (a) separation zones on floor; $(b)$ diagram of SBLI structure on the central symmetry plane. 
shows the opposite behaviour. This can be explained by two factors as shown in figure 5.3b. First, the corner 'shock' is of relatively limited strength and consists of a series of compression waves rather than a single strong shock. These compression waves, now arriving ahead of the main interaction, act to provide a gentle 'pre-compression' and smear the adverse pressure gradient through the SBLI. Second, the corner shock is followed by expansion waves which now reach the symmetry plane within the interaction zone (as seen in figure 4.37 by the strong re-acceleration of the flow above the interaction region for $x_{\text {block }}=85 \mathrm{~mm}$ and $x_{\text {block }}=75 \mathrm{~mm}$ ). This means they can now relieve the overall pressure rise which reduces the separation length $L_{s e p}$. And, the re-acceleration of the flow also helps to give the dividing streamline/separated shear layer momentum to overcome reattachment.

\subsection{Summary}

The mechanisms discussed above suggest that a typical oblique SBLI in a rectangular channel can be divided into several zones (I-V) depending on the relative position of the corner waves and the main incident shock wave. Based on the influence of corner waves the dependence of $L_{\text {sep }}$ and $L_{f}$ on the corner shock crossing point, as plotted in figures 4.41 and 4.42 , is properly explained. A nominal-two-dimensional central separation occurs for the cases with relatively 'mild' corner effects (correspond to very large facilities or very small viscous regions), where the corner waves arrive far downstream of the interaction zone and leave a sizeable region in the centre-span outside the influence of corner effects. For the interactions with corner shocks crossing inside the separated region, the additional shock-imposed pressure rise leads to an increase in separation length $L_{\text {sep }}$, while no or only part of the beneficial expansion waves reach the interaction. In cases with a $L_{s e p}$ reduced beyond the equivalent two-dimensional value, the effects of detrimental corner shocks and beneficial expansions on the interaction reverse. The corner shocks intersect ahead of the incident shock and smear the overall pressure jump over a larger area. This is aided by the stronger re-acceleration from the corner expansions now arriving inside the interaction.

The footprint of the corner shocks has also been applied to the surface flow visualisation of previous numerical studies with different wedge angles, Mach numbers and aspect ratios, such as those performed by Benek et al. [12] and Wang et al. [134]. The relative position of the corner shock crossing point and the main interaction domain is shown to capture the extent of sidewall effects, and correctly predict the general behaviour of central separations.

The underlying physics indicate that all experiments are likely to experience corner effects and a direct comparison with two-dimensional numerical computations is only correct for flows with corner separations relatively small compared to the facility width. The analysis 
further suggests that to accurately predict the separation behaviour, the corner shock crossing point can be used to determine the relative importance of three-dimensional effects. 


\section{Chapter 6}

\section{Conclusions and future work}

\subsection{Conclusions}

When shock wave/boundary layer interactions occur in a rectangular duct, a region of shockinduced separation is often observed in each of the streamwise corners. A review of the existing literature has suggested that the flow along the centre-span of the channel floor can be 'contaminated' by the presence of corner separations. Any interplay between corner separations and flow away from the corner is very important for fundamental investigations conducted in supersonic wind tunnel facilities with rectangular cross-sections. These features are also relevant to mixed-compression inlets for supersonic aircraft, which share the same geometry.

However, the understanding of the flow physics accounting for corner effects remains very nascent. In particular, for oblique reflecting interactions in rectangular channels, the published literature is unable to explain how corner separations can alter the nominally two-dimensional SBLIs and by what mechanism this is achieved. The aim of this thesis is therefore set out to address this gap in the knowledge regarding such an arrangement.

Experiments have been conducted to study an oblique SBLI in the Cambridge supersonic wind tunnel which has a rectangular cross-section. A nominal freestream Mach number of 2.5 is used for all experiments with a unit Reynolds number of $40 \times 10^{6} \mathrm{~m}^{-1}$. A flow deflection of $8^{\circ}$ is generated by a wedge mounted at the tunnel ceiling. The adverse pressure gradient resulting from the oblique shock reflection on the floor of the working section is sufficient to cause substantial separations both in the corners and around the centre-span.

Investigations have been performed to explore effective control methods to reduce corner effects in an oblique SBLI. It is seen that the streamwise placement of a localised corner suction relative to the main oblique interaction affects the control effectiveness, with suction placed downstream and across the interaction performing better than suction upstream. 
However even at a favourable bleed position, the corner separations are only mildly reduced. The slight changes are a consequence of the extremely low suction mass flow rates applied, especially at the bleed location upstream of the incident shock (only $0.2 \%$ of the total mass flow through the tunnel working section). The suction strength is restricted by the low local surface pressures associated with the high Mach numbers, making it very difficult to achieve the desired bleed rate for these high-speed flows. Micro-vanes placed in the corner regions ahead of the incident shock are found to reduce the size of corner separations considerably, by approximately $45 \%$ compared to the baseline flow. A combination of micro-VGs and suction works most effectively to mitigate the corner effects, giving a $65 \%$ decrease from the uncontrolled corner separation magnitude. This is achieved with only a small percentage of mass flow $(0.3 \%)$ being removed.

In contrast, to increase the magnitude of corner separations to varied extents, two sets of obstacles with different sizes are positioned in the corner regions. The local separation has been increased in spanwise extent by $18 \%$ and $50 \%$ respectively compared to the undisturbed flow.

These flow control methods are then applied to modify the magnitude and onset of corner separations, to produce corner effects varying in relative size from moderate to significant. It is observed that changes to the geometry of corner separations can strongly affect the size and shape of separation in the centre of the tunnel floor. A decrease in the size of the corner separation generates a more two-dimensional flow field. In this case, the central separation covers a wider spanwise extent with relatively straight separation and reattachment lines. An increase in the size of the corner separation drastically increases the three-dimensionality of the overall flowfield. The separation length measured along the centreline is observed to be considerably altered, presenting an increasing and then decreasing trend as the onset of corner separation shifts upstream. The surface flow topology of the separated region in the centre is seen to transit from the quasi-two-dimensional 'owl-face of the first kind' to the more complicated second kind.

The primary mechanism behind this sidewall effect appears to be the waves induced by corner separations, which act to alter the separated interaction wave structure and in turn influence the adverse pressure gradient imposed upon boundary layer elsewhere. The blockage effect of the corner separation first deflects the supersonic flow away from the corner through a series of compression waves, or 'corner shock waves', and then returns the flow towards the streamwise direction by an expansion fan. The impact of these corner waves on the central separation is found to be well illustrated by the position of the corner shock crossing point. If the corner shocks reach the symmetry plane downstream of the interaction zone, the centreline separation is unaffected. If the crossing point is inside the separated flow, 
an increase in separation length and a more three-dimensional separation bubble can result because of the detrimental effect of the corner shocks. In cases where the corner shocks are shifted further upstream and intersected ahead of the interaction, a reduction in centreline separation length even beyond the two-dimensional value is observed due to the beneficial effect of the corner expansion waves. The experimental conditions tested show that the presence of corner effects can cause the centreline separation length to vary from $36 \%$ to $180 \%$ of the nominally two-dimensional baseline value.

\subsection{Recommendations and future work}

The results illustrate the significance of corner effects for oblique SBLIs in rectangular channels. For wind tunnel investigations relating to fundamental SBLIs and control devices, examining the centreline flow without accounting for corner separations can therefore result in inaccurate measurements and interpretations. For numerical studies on reflecting shock interaction, a correct prediction of the corner flow geometry is recommended as a prerequisite to the correct prediction of the main interaction behaviour in the centre of a duct. However due to the limited capability of reliably modelling corner flows, if centreline flow is of primary interest, the corner separation is suggested to be treated as a 'blockage' shaped to induce compression waves followed by an expansion fan for simplicity.

While the current study has only investigated one particular free-stream Mach number and oblique shock strength, it is the author's view that the fundamental wave interactions and their effect on the overall flow topology is similar for all oblique shock reflections, and this allows a few basic 'predictions'. Given that the corner shock crossing point has been identified as a critical parameter, it is suggested that interactions with greater Mach number inflows are likely to exhibit more 'two-dimensional' behaviour as the wave angle would be expected to decrease with increasing Mach numbers. On the other hand, stronger shock strengths are expected to increase the size of corner separations, which would lead to a more upstream position of the corner waves and thus promote more 'three-dimensional' interactions.

The generality of the experimental findings can be tested by future research for different shock strengths and incoming boundary-layer conditions. For a further insight into the impact of extensive corner separations, corner fillets can be applied to replicate the corner obstacle effect. A new LDA seeding system introducing droplets into the corner region would allow velocity measurements inside the corners. These measurements would be very useful to determine the flow structure developed inside a corner separation, thus providing a more accurate indication of the blockage generated by corner flow separations. 



\section{References}

[1] Ackeret, J., Feldmann, F., and Rott, N. (1947). Investigations of compression shocks and boundary layers in gases moving at high speed. NACA TM.

[2] Adamson, T. C. and Messiter, A. F. (1980). Analysis of two-dimensional interactions between shock waves and boundary layers. Annual Review of Fluid Mechanics, 12(1):103138.

[3] Agostini, L., Larchevêque, L., Garnier, E., and De Martel, E. (2012). Numerical study of three-dimensional modulations in a shock-induced separation. Progress in Flight Physics, 3:157-168.

[4] Anderson, J. D. (2003). Modern Compressible Flow with Historical Perspective. McGraw Hill.

[5] Ashill, P., Fulker, J., and Hackett, K. (2005). A review of recent developments in flow control. The Aeronautical Journal, 109(1095):205-232.

[6] Atkin, C. and Squire, C. (1992). A study of the interaction of a normal shock wave with a turbulent boundary layer at Mach numbers between 1. 30 and 1. 55. European Journal of Mechanics, B/Fluids, 11(1):93-118.

[7] Babinsky, H. and Harvey, J. K. (2011). Shock wave-boundary-layer interactions. Cambridge University Press.

[8] Babinsky, H., Li, Y., and Pitt Ford, C. W. (2009). Microramp control of supersonic oblique shock-wave/boundary-layer interactions. AIAA Journal, 47(3):668-675.

[9] Babinsky, H. and Ogawa, H. (2008). SBLI control for wings and inlets. Shock Waves, 18(2):89.

[10] Babinsky, H., Oorebeek, J., and Cottingham, T. (2013). Corner effects in reflecting oblique shock-wave/boundary-layer interactions. 51st AIAA Aerospace Sciences Meeting, page 859 .

[11] Benek, J. A., Suchyta, C. J., and Babinsky, H. (2013). The effect of tunnel size on incident shock boundary layer interaction experiments. 51st AIAA Aerospace Sciences Meeting, page 862.

[12] Benek, J. A., Suchyta, C. J., and Babinsky, H. (2016). Simulations of incident shock boundary layer interactions. 54th AIAA Aerospace Sciences Meeting, page 0352. 
[13] Birkemeyer, J., Rosemann, H., and Stanewsky, E. (2000). Shock control on a swept wing. Aerospace Science and Technology, 4:147-156.

[14] Blinde, P. L., Humble, R. A., Van Oudheusden, B. W., and Scarano, F. (2009). Effects of micro-ramps on a shock wave/turbulent boundary layer interaction. Shock Waves, 19(6):507.

[15] Bodner, J., Greber, I., Davis, D., and Hingst, W. (1996). Experimental investigation of the effect of a single bleed hole on a supersonic turbulent boundary-layer. 32nd Joint Propulsion Conference and Exhibit, page 2797.

[16] Bogdonoff, S. (1955). Separation of a supersonic turbulent boundary layer. Journal of the Aeronautical Sciences, 22(6):414-430.

[17] Bogdonoff, S. and Poddar, K. (1991). An exploratory study of a three-dimensional shock wave turbulent boundary layer interaction in a corner. 29th Aerospace Sciences Meeting, page 525.

[18] Bourgoing, A. and Reijasse, P. (2005). Experimental analysis of unsteady separated flows in a supersonic planar nozzle. Shock Waves, 14(4):251-258.

[19] Bradshaw, P. (1987). Turbulent secondary flows. Annual Review of Fluid Mechanics, 19(1):53-74.

[20] Bragg, S. L. (1960). Effect of compressibility on the discharge coefficient of orifices and convergent nozzles. Journal of Mechanical Engineering Science, 2(1):35-44.

[21] Brayton, D. (1974). Small particle signal characteristics of a dual-scatter laser velocimeter. Applied optics, 13(10):2346-2351.

[22] Brown, L. and Naughton, W. (1999). The thin oil film equation. Technical report, NASA/TM-208767.

[23] Bruce, P. J. K. (2008). Transonic shock/boundary layer interactions subject to downstream pressure perturbations. $\mathrm{PhD}$ thesis, University of Cambridge.

[24] Bruce, P. J. K., Babinsky, H., Tartinville, B., and Hirsch, C. (2011a). Corner effect and asymmetry in transonic channel flows. AIAA Journal, 49(11):2382-2392.

[25] Bruce, P. J. K., Burton, D. M. F., Titchener, N. A., and Babinsky, H. (2011b). Corner effect and separation in transonic channel flows. Journal of Fluid Mechanics, 679:247262.

[26] Brusniak, L. and Dolling, D. S. (1994). Physics of unsteady blunt-fin-induced shock wave/turbulent boundary layer interactions. Journal of Fluid Mechanics, 273:375-409.

[27] Bur, R., Benay, R., Galli, A., and Berthouze, P. (2006). Experimental and numerical study of forced shock-wave oscillations in a transonic channel. Aerospace science and technology, 10(4):265-278.

[28] Burton, D. and Babinsky, H. (2012). Corner separation effects for normal shock wave/turbulent boundary layer interactions in rectangular channels. Journal of Fluid Mechanics, 707:287-306. 
[29] Burton, D., Babinsky, H., and Bruce, P. (2010). Experimental investigation into parameters governing corner interactions for transonic shock-wave/boundary-layer interactions. 48th AIAA Aerospace Sciences Meeting and Exhibition, page 871.

[30] Caruana, D., Mignosi, A., Corrège, M., Le Pourhiet, A., and Rodde, A. (2005). Buffet and buffeting control in transonic flow. Aerospace Science and Technology, 9(7):605-616.

[31] Chapman, D. R., Kuehn, D. M., and Larson, H. K. (1958). Investigation of separated flows in supersonic and subsonic streams with emphasis on the effect of transition. NACA Report.

[32] Chriss, R., Hingst, W., Strazisar, A., and Keith, T. (1990). An LDA investigation of the normal shock wave boundary layer interaction. La Recherche Aérospatiale (English Edition), pages 1-15.

[33] Coles, D. (1956). The law of the wake in the turbulent boundary layer. Journal of Fluid Mechanics, 1(2):191-226.

[34] Colliss, S. P. (2014). Vortical structures on three-dimensional shock control bumps. $\mathrm{PhD}$ thesis, University of Cambridge.

[35] Coschignano, A. (2018). Normal shock wave-boundary layer interactions in transonic intakes at incidence. $\mathrm{PhD}$ thesis, University of Cambridge.

[36] Dandois, J. (2014). Improvement of corner flow prediction using the quadratic constitutive relation. AIAA Journal, 52(12):2795-2806.

[37] Davidson, T. (2016). Effect of incoming boundary layer state on flow development downstream of normal shock wave-boundary layer interactions. PhD thesis, University of Cambridge.

[38] Davis, D. and Gessner, F. (1989). Further experiments on supersonic turbulent flow development in a square duct. AIAA Journal, 27(8):1023-1030.

[39] Davis, D., Gessner, F., and Kerlick, G. (1986). Experimental and numerical investigation of supersonic turbulent flow through a square duct. AIAA Journal, 24(9):1508-1515.

[40] Délery, J. and Bur, R. (2000). The physics of shock wave/boundary layer interaction control: last lessons learned. In ECCOMAS, 11-14 September, Barcelona Spain, 181.

[41] Délery, J., Marvin, J., and Reshotko, E. (1986). Shock-wave boundary layer interactions. NASA STI/Recon Technical Report N, 86.

[42] Délery, J. M. (1985). Shock wave/turbulent boundary layer interaction and its control. Progress in Aerospace Sciences, 22(4):209-280.

[43] Délery, J. M. (2001). Robert Legendre and Henri Werlé: toward the elucidation of three-dimensional separation. Annual Review of Fluid Mechanics, 33(1):129-154.

[44] Délery, J. M. (2013). Three-dimensional separated flow topology: critical points, separation lines and vortical structures. ISTE and Wiley. 
[45] Délery, J. M. and Dussauge, J.-P. (2009). Some physical aspects of shock wave/boundary layer interactions. Shock Waves, 19(6):453-468.

[46] Deleuze, J. (1995). Structure d'une couche limite turbulente soumise à une onde de choc incidente. $\mathrm{PhD}$ thesis, UniversitèAix-Marseille II.

[47] Doerffer, P., Hirsch, C., Dussauge, J.-P., Babinsky, H., and Barakos, G. N. (2010). Unsteady effects of shock wave induced separation, volume 114. Springer Science \& Business Media.

[48] Doerffer, P. and Szwaba, R. (2009). UFAST experimental data bank - unsteady effects of shock wave induced separation. UFAST Experimental Data Bank - Unsteady Effects of Shock Wave Induced Separation, pages 131-177.

[49] Dupont, P., Haddad, C., Ardissone, J. P., and Debieve, J. F. (2005). Space and time organisation of a shock wave/turbulent boundary layer interaction. Aerospace Science and Technology, 9(7):561-572.

[50] Durst, F., Melling, A., and Whitelaw, J. (1981). Principles and practice of laser doppler anemometry.

[51] Eagle, W. E. and Driscoll, J. F. (2014). Shock wave-boundary layer interactions in rectangular inlets: three-dimensional separation topology and critical points. Journal of Fluid Mechanics, 756:328-353.

[52] Fisher, S. (1986). Three-dimensional flow effects in a two-dimensional supersonic air intake. Journal of Propulsion and Power, 2(6):546-551.

[53] Frey, M. and Hagemann, G. (2000). Restricted shock separation in rocket nozzles. Journal of Propulsion and Power, 16(3):478-484.

[54] Fukuda, M., Hingst, W., and Reshotko, E. (1977). Bleed effects on shock/boundarylayer interactions in supersonic mixed compression inlets. Journal of Aircraft, 14(2):151156.

[55] Gadd, G., Holder, D. W., and Regan, J. (1954). An experimental investigation of the interaction between shock waves and boundary layers. Proceedings of The Royal Society A: Mathematical, Physical and Engineering Sciences, 226(1165):227-253.

[56] Gaitonde, D. and Shang, J. S. (1995). Structure of a turbulent double-fin interaction at Mach 4. AIAA Journal, 33(12):2250-2258.

[57] Gaitonde, D. V. (2015). Progress in shock wave/boundary layer interactions. Progress in Aerospace Sciences, 72:80-99.

[58] Garnier, E. (2009). Stimulated detached eddy simulation of three-dimensional shock/boundary layer interaction. Shock Waves, 19(6):479.

[59] Gessner, F. (1973). The origin of secondary flow in turbulent flow along a corner. Journal of Fluid Mechanics, 58(1):1-25.

[60] Giepman, R. H. M. (2016). Flow control for oblique shock wave reflections. $\mathrm{PhD}$ thesis, Technische Universiteit Delft. 
[61] Godard, G. and Stanislas, M. (2006). Control of a decelerating boundary layer. part 1: Optimization of passive vortex generators. Aerospace Science and Technology, 10(3):181191.

[62] Green, J. (1970a). Interactions between shock waves and turbulent boundary layers. Progress in Aerospace Sciences, 11:235-340.

[63] Green, J. (1970b). Reflexion of an oblique shock wave by a turbulent boundary layer. Journal of Fluid Mechanics, 40(1):81-95.

[64] Gregory, J., Asai, K., Kameda, M., Liu, T., and Sullivan, J. (2008). A review of pressuresensitive paint for high-speed and unsteady aerodynamics. Proceedings of the Institution of Mechanical Engineers, Part G: Journal of Aerospace Engineering, 222(2):249-290.

[65] Handa, T., Masuda, M., and Matsuo, K. (2005). Three-dimensional normal shockwave/boundary-layer interaction in a rectangular duct. AIAA Journal, 43(10):2182-2187.

[66] Harloff, G. J. and Smith, G. E. (1996). Supersonic-inlet boundary-layer bleed flow. AIAA Journal, 34(4):778-785.

[67] Hassal, M. (2017). A practical guide to turbulence and ow measurements by thermal anemometry (CTA), laser-doppler anemometry (LDA) and particle-image velocimetry (PIV). Technical Report, Engineering Sciences Data Unit (ESDU).

[68] Hayashi, M., Aso, S., and Tan, A. (1989). Fluctuation of heat transfer in shock wave/turbulent boundary-layer interaction. AIAA Journal, 27(4):399-404.

[69] Helmer, D. B., Campo, L. M., and Eaton, J. K. (2012). Three-dimensional features of a Mach 2.1 shock/boundary layer interaction. Experiments in Fluids, 53(5):1347-1368.

[70] Henderson, L. (1967). The reflexion of a shock wave at a rigid wall in the presence of a boundary layer. Journal of Fluid Mechanics, 30(4):699-722.

[71] Hill, P. G. and Peterson, C. R. (1992). Mechanics and thermodynamics of propulsion. Reading, MA, Addison-Wesley Publishing Co.

[72] Hingst, W. and Tanji, F. (1983). Experimental investigation of a two-dimensional shock-turbulent boundary layer interaction with bleed. 21st Aerospace Sciences Meeting, page 135 .

[73] Holden, H. A. and Babinsky, H. (2007). Effect of microvortex generators on seperated normal shock/boundary layer interactions. Journal of Aircraft, 44(1):170-174.

[74] Kooi, J. (1978). Influence of free-stream Mach number on transonic shock-wave boundary-layer interaction. Technical report, Nationaal Lucht-en Ruimtevaartlaboratorium.

[75] Krein, A. and Williams, G. (2012). Flightpath 2050: Europe's vision for aeronautics. Innovation for Sustainable Aviation in a Global Environment: Proceedings of the Sixth European Aeronautics Days, pages 63-71.

[76] Kussoy, M. I., Horstoman, K. C., and Horstman, C. (1993). Hypersonic crossing shock-wave/turbulent-boundary-layer interactions. AIAA Journal, 31(12):2197-2203. 
[77] Lai, W., Shakal, J., and Troolin, D. (2013). Accuracy, resolution, and repeatability of powersight PDPA and LDV systems. TSI Technical Note P, (5001520):A4.

[78] Laurent, H. (1996). Turbulence d'une interaction onde de choc/couche limite sur une paroi plane adiabatique ou chaufèe. $\mathrm{PhD}$ thesis, UniversitèAix-Marseille II.

[79] Law, C. H. (1976). Supersonic shock wave turbulent boundary-layer interactions. AIAA Journal, 14(6):730-734.

[80] Legendre, R. (1977). Streamlines in permanent flows: Detachment and separation. La Recherche Aérospatiale, (1977-6).

[81] Lin, J. C. (2002). Review of research on low-profile vortex generators to control boundary-layer separation. Progress in Aerospace Sciences, 38(4-5):389-420.

[82] Lineberger, R. S. and Hussain, A. (2018). 2018 global aerospace and defense industry outlook. Deloitte Executive Report.

[83] Liu, T., Guille, M., and Sullivan, J. P. (2001). Accuracy of pressure-sensitive paint. AIAA Journal, 39(1):103-112.

[84] Mani, M., Babcock, D., Winkler, C., and Spalart, P. (2013). Predictions of a supersonic turbulent flow in a square duct. 51st AIAA Aerospace Sciences Meeting, page 860.

[85] Matheis, J. and Hickel, S. (2015). On the transition between regular and irregular shock patterns of shock-wave/boundary-layer interactions. Journal of Fluid Mechanics, 776:200-234.

[86] McKeon, B., Comte-Bellot, G., Foss, J., Westerweel, J., Scarano, F., Tropea, C., Meyers, J., Lee, J., Cavone, A., Schodl, R., et al. (2007). Velocity, vorticity, and Mach number. Springer Handbook of Experimental Fluid Mechanics, pages 215-471.

[87] McLachlan, B. G. and Bell, J. H. (1995). Pressure-sensitive paint in aerodynamic testing. Experimental Thermal and Fluid Science, 10(4):470-485.

[88] McLaughlin, D. and Tiederman, W. (1973). Biasing correction for individual realization of laser anemometer measurements in turbulent flows. The Physics of Fluids, 16(12):20822088.

[89] Mendoza, D. R. (1997). Limiting Mach number for quantitative pressure-sensitive paint measurements. AIAA Journal, 35(7):1240-1241.

[90] Merzkirch, W. (2012). Flow Visualization. Elsevier.

[91] Mie, G. (1908). Articles on the optical characteristics of turbid tubes, especially colloidal metal solutions. Ann. Phys, 25(3):377-445.

[92] Mojola, O. and Young, A. (1971). An experimental investigation of the turbulent boundary layer along a streamwise corner. AGARD Conference, Turbulent shear flows, (93):12-1.

[93] Molton, P., Dandois, J., Lepage, A., Brunet, V., and Bur, R. (2013). Control of buffet phenomenon on a transonic swept wing. AIAA Journal, 51(4):761-772. 
[94] Morajkar, R. R., Driscoll, J. F., and Gamba, M. (2015). Experimental study of supersonic turbulent corner flow evolution in a low aspect ratio rectangular channel. 53rd AIAA Aerospace Sciences Meeting, page 0542.

[95] Morkovin, M. V. (1962). Effects of compressibility on turbulent flows. Mécanique de la Turbulence, 367:380.

[96] Musker, A. J. (1979). Explicit expression for the smooth wall velocity distribution in a turbulent boundary layer. AIAA Journal, 17(6):655-657.

[97] Nolan, W. R. (2013). A study of vortex generator behaviour. PhD thesis, University of Cambridge.

[98] Ogawa, H. (2006). Experimental and analytical study of transonic shockwave/boundary layer interaction control with three-dimensional bumps. PhD thesis, University of Cambridge.

[99] Oorebeek, J. (2014). Comparison of distributed suction and vortex generator flow control for a transonic diffuser. PhD thesis, University of Cambridge.

[100] Oskam, B., Vas, I., and Bogdonoff, S. (1977). An experimental study of threedimensional flow fields in an axial corner at Mach 3. 10th Fluid and Plasmadynamics Conference, page 689.

[101] Pearcey, H. (1959). Some effects of shock-induced separation of turbulent boundary layers in transonic flow past aerofoils. HM Stationery Office.

[102] Pearcey, H. (1961). Introduction to shock-induced separation and its prevention by design and boundary layer control. In Boundary layer and flow control, pages 1166-1344. Elsevier.

[103] Pearcey, H., Osborne, J., and Haines, A. (1968). The interaction between local effects at the shock and rear separation-a source of significant scale effects in wind-tunnel tests on aerofoils and wings. Technical report, AGARD CP-35 Transonic Aerodynamics.

[104] Perry, A. E. and Hornung, H. (1984). Some aspects of three-dimensional separation. ii-vortex skeletons. Zeitschrift fur Flugwissenschaften und Weltraumforschung, 8:155160 .

[105] Piponniau, S., Dussauge, J., Debieve, J., and Dupont, P. (2009). A simple model for low-frequency unsteadiness in shock-induced separation. Journal of Fluid Mechanics, 629:87-108.

[106] Prandtl, L. (1963). The essentials of fluid dynamics. Blackie \& Son Limited.

[107] Reda, D. C. and Murphy, J. D. (1973). Shock wave/turbulent boundary-layer interactions in rectangular channels. AIAA Journal, 11(2):139-140.

[108] Sajben, M. (1993). Uncertainty estimates for pressure sensitive paint measurements. AIAA journal, 31(11):2105-2110. 
[109] Sajben, M., Morris, M., Bogar, T., and Kroutil, J. (1991). Confined normalshock/turbulent-boundary-layer interaction followed by an adverse pressure gradient. AIAA Journal, 29(12):2115-2123.

[110] Sami, K. (2011). Physics of Three-Dimensional Normal Shock Wave/Turbulent Boundary Layer Interactions in Rectangular Channels. $\mathrm{PhD}$ thesis, University of Cambridge.

[111] Sawyer, W. and Long, C. J. (1982). A study of normal shock-wave turbulent boundarylayer interactions at Mach numbers of 1.3, 1.4 and 1.5. Technical report, RAE.

[112] Seddon, J. (1960). The flow produced by interaction of a turbulent boundary layer with a normal shock wave of strength sufficient to cause separation. TIL.

[113] Seebaugh, W. and Childs, M. (1970). Conical shock-wave turbulent boundary-layer interaction including suction effects. Journal of Aircraft, 7(4):334-340.

[114] Settles, G. S., Fitzpatrick, T. J., and Bogdonoff, S. M. (1979). Detailed study of attached and separated compression corner flowfields in high reynolds number supersonic flow. AIAA Journal, 17(6):579-585.

[115] Shang, J., Hankey Jr, W., and Law, C. (1976). Numerical simulation of shock waveturbulent boundary-layer interaction. AIAA Journal, 14(10):1451-1457.

[116] Shapiro, A. H. (1954). The dynamics and thermodynamics of compressible fluid flow (vol2). The Ronald Press Company.

[117] Shaw, R. (1959). The influence of hole dimensions on static pressure measurements. Journal of Fluid Mechanics, 7(4):550-564.

[118] Smith, D. R. and Smits, A. J. (1997). The effects of successive distortions on a turbulent boundary layer in a supersonic flow. Journal of Fluid Mechanics, 351:253-288.

[119] Spalart, P. R. (2000). Strategies for turbulence modelling and simulations. International Journal of Heat and Fluid Flow, 21(3):252-263.

[120] Squire, L. C. (1961). The motion of a thin oil sheet under the steady boundary layer on a body. Journal of Fluid Mechanics, 11(2):161-179.

[121] Sun, C.-C. and Childs, M. E. (1973). A modified wall wake velocity profile for turbulent compressible boundary layers. Journal of Aircraft, 10(6):381-383.

[122] Sun, C.-C. and Childs, M. E. (1977). An experimental investigation of shock waveturbulent boundary layer interactions with and without boundary layer suction: A data summary report. NASA STI/Recon Technical Report N, 77.

[123] Szumowski, A. and Wojciechowski, J. (2005). Use of vortex generators to control internal supersonic flow separation. AIAA Journal, 43(1):216-218.

[124] Thom, A. and Apelt, C. J. (1958). The pressure in a two-dimensional static hole at low Reynolds numbers. HM Stationery Office. 
[125] Thomas, P., Buetefisch, K.-A., and Sauerland, K. (1993). On the motion of particles in a fluid under the influence of a large velocity gradient. Experiments in Fluids, 14(12):42-48.

[126] Thomke, G. and Roshko, A. (1969). Incipient separation of a turbulent boundary layer at high reynolds number in two-dimensional supersonic flow over a compression corner. NASA Technical Report.

[127] Titchener, N. and Babinsky, H. (2013). Shock wave/boundary-layer interaction control using a combination of vortex generators and bleed. AIAA Journal, 51(5):1221-1233.

[128] Titchener, N. and Babinsky, H. (2015). A review of the use of vortex generators for mitigating shock-induced separation. Shock Waves, 25(5):473-494.

[129] Titchener, N., Babinsky, H., and Loth, E. (2013). The effects of various vortex generator configurations on a normal shock wave/boundary layer interaction. 51st AIAA Aerospace Sciences Meeting, page 18.

[130] Titchener, N., Bruce, P., and Babinsky, H. (2011). An experimental investigation of corner bleed applied to a normal shock-wave/boundary-layer interaction and diffuser. 49th AIAA Aerospace Sciences Meeting, page 69.

[131] Titchener, N., Colliss, S., and Babinsky, H. (2015). On the calculation of boundarylayer parameters from discrete data. Experiments in Fluids, 56(8):159.

[132] Unalmis, O. and Dolling, D. (1996). On the possible relationship between low frequency unsteadiness of shock-induced separated flow and goertler vortices. page 2002.

[133] van Driest, E. R. (1951). Turbulent boundary layer in compressible fluids. Journal of the Aeronautical Sciences, 18(3):145-160.

[134] Wang, B., Sandham, N. D., Hu, Z., and Liu, W. (2015). Numerical study of oblique shock-wave/boundary-layer interaction considering sidewall effects. Journal of Fluid Mechanics, 767:526-561.

[135] Wang, K. (1972). Separation patterns of boundary layer over an inclined body of revolution. AIAA Journal, 10(8):1044-1050.

[136] Weber, A., Schreiber, H.-A., Fuchs, R., and Steinert, W. (2002). 3D transonic flow in a compressor cascade with shock-induced corner stall. Journal of Turbomachinery, 124:358-366.

[137] White, F. M. (2006). Viscous fluid flow, volume 3. McGraw Hill.

[138] Willis, B., Davis, D., and Hingst, W. (1995). Flow coefficient behavior for boundary layer bleed holes and slots. 33rd AIAA Aerospace Sciences Meeting, page 31.

[139] Wu, J., Tramel, R., Zhu, F., and Yin, X. (2000). A vorticity dynamics theory of three-dimensional flow separation. Physics of Fluids, 12(8):1932-1954.

[140] Wu, M. and Martin, M. P. (2008). Analysis of shock motion in shock wave and turbulent boundary layer interaction using direct numerical simulation data. Journal of Fluid Mechanics, 594:71-83. 
